\title{
CAEP/ACMU 2021 Scientific Abstracts
}

June 15 - 17th, 2021

\section{Contents}

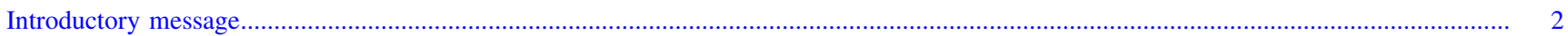

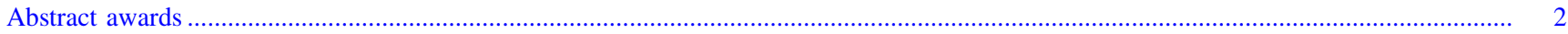

Plenary

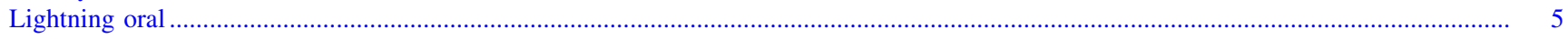

Moderated poster

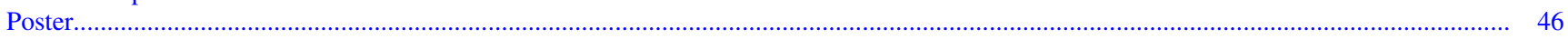

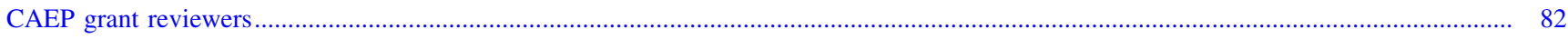

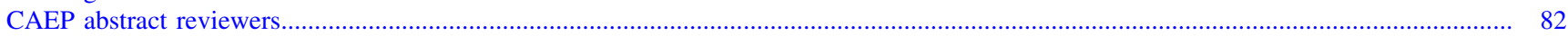

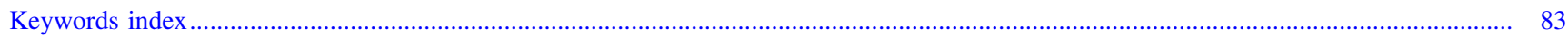

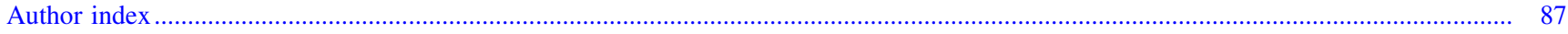




\section{Introductory message}

Research in emergency medicine (EM) guides improvements in emergency patient care. Research helps to identify and standardize the best care to optimize patient outcomes.

Fostering a rich research environment requires funding, education, and a rigorous peer-review process. The CAEP Research Committee is pleased to support the development of EM-related research skills across Canada by administering two annual programs: the CAEP Grant Competition and the CAEP Abstract Competition.

Abstracts are the core of the research competition. This year for CAEP 2021 which will be held virtually, we had 3 submission categories: research, education, and quality improvement and patient safety, as well as subcategories for quantitative research, qualitative research and education innovations. We received 215 abstract submissions from EM researchers from across Canada and internationally, where the top-ranked abstracts will be presented at the research plenary session. In addition, the best resident, pediatric, new investigator, education innovation, venous thromboembolism (VTE), quality improvement and patient safety, and medical student abstracts submitted by CAEP members are awarded financially to subsidize conference registration.

CAEP introduced the new CIHR-ICRH/CAEP Mid-Career Lecturer Award in Emergency Medicine in 2020; a 3-year partnership which allows the recipient to deliver a plenary presentation at the annual CAEP conference. The purpose of establishing this annual award by the CIHR-ICRH and CAEP is to recognize an individual's outstanding contribution to the advancement of emergency medicine in Canada and internationally at the mid-point of their career. With the annual conference being cancelled in 2020, our inaugural recipient will present at CAEP 2021.

The hours of work that our volunteer reviewers contribute is critical to the success of the Research Committee activities during the busy abstract and grant competitions. Each submission is thoroughly read, reviewed, and scored by at least three experienced reviewers. The Research Committee would like to thank the reviewers for their contribution and recognize their commitment to support EM research.

The Research Committee would like to acknowledge the substantial effort of CAEP staff, particularly Shanna Scarrow, Kelly Wyatt and Gail Chapman, in coordinating the grant and abstract competitions, and in preparing the CAEP conference research program and CJEM research supplement.

Disclaimer: The large number of submitted abstracts and the deadlines associated with publication do not permit the author communication, abstract revisions, or CJEM editorial review. The abstracts are presented, as they were submitted to the Research Committee. Only the author affiliation supplied by the presenting author is specified.

Note: The CAEP 2021 final program (via the conference platform) contains the scheduled times for the abstract presentations.

Andrew McRae, MD PhD FRCPC

CAEP/ACMU Research Committee Chair

Justin Yan, MD MSc FRCPC

CAEP/ACMU Research Committee Abstract Competition Lead

\section{Abstract awards}

First place, Plenary Presentation, Grant Innes Research Paper and Presentation Award

David Barbic

PL01 Rapid agitation control with ketamine in the emergency department: a double blind, randomized controlled trial

Second place, Plenary Presentation, Top New Investigator Research Abstract Award

Keerat Grewal

PL02 Intracranial hemorrhage after head injury among anticoagulated elderly patients seen in the emergency department: a populationbased cohort study

Third place, Plenary Presentation Christian Vaillancourt

PL03 Strategy empowering paramedics to assess low-risk trauma patients with the Canadian c-spine rule and selectively transport them without immobilization: a pragmatic cluster randomized stepped wedge patient-informed multi-center trial

\section{Fourth place, Plenary Presentation} Natalie Le Sage

PL04 PoCS Rule: derivation and validation of a clinical decision rule for early detection of persistent symptoms after a mild traumatic brain injury

Top Medical Student Abstract Award Marika Moskalyk

LO61 Assessing the learning impact of the Northern City of Heroes public cardiopulmonary resuscitation exhibit at Science North in Sudbury, Ontario

Top Resident Research Abstract Award Kumait Al Lawati

LO36 Efficacy and safety of tranexamic acid in acute traumatic brain injury: a systematic review and meta-analysis of randomized controlled trials

\section{Top Pediatric Research Abstract Award} Dominic Chung

LO05 A longitudinal, population-based study on mental health outcomes in child survivors of traumatic injury compared with matched controls

\section{Top QIPS Abstract Award} Jeff Yoo

LO12 A quality improvement project to enhance emergency department intubation performance and decrease complications during the COVID-19 pandemic

Top Education Innovation Abstract Finalists Wilson Lam

LO49 The virtual delivery of a national rural medical education and in-situ simulation curriculum 


\section{Ahmed Taher}

LO50 HiQuiPs - health informatics quality improvement patient safety: a novel free open access medical education resource

\section{Sarah Foohey}

LO51 Developing the Virtual Resus Room: usability, accessibility, and applicability of a virtual simulation alternative for teaching and learning

\section{CAEP-CanVECTOR Research Abstract Awards \\ Keerat Grewal}

LO41 Venous thromboembolism in patients discharged from the emergency department with ankle fractures: a population-based cohort study

\section{Alan Tan}

L069 Careful anticoagulation review in emergency medicine: the CARe-EM study

\section{Choosing Wisely Canada Abstract Awards Sameer Masood}

LO11 A quality improvement initiative to provide COVID-19 patients with rapid follow up via a novel multi-disciplinary virtual clinic following an emergency department visit

\section{Aikta Verma}

LO14 Reducing hospital admissions using an interprofessional team in an academic emergency department

\section{CAEP Resident Research Abstract Awards Elizabeth Williams}

LO07 Identification of pediatric emergency department patients for referral to rapid-access substance use services

\section{Hashim Kareemi}

LO25 Machine Learning versus usual care for diagnostic and prognostic prediction in the emergency department: a systematic review

\section{Sally Kang}

LO45 Patient characteristics and outcomes associated with cancer diagnosis in the emergency department: a systematic review

\section{Lawrence Yau}

LO53 Comparing the frequency of ST-elevation myocardial infarction bypasses and their associated short term outcomes during and before the COVID-19 pandemic

\section{Erin Creasor}

LO70 Diagnostic accuracy of non-invasive imaging modalities versus direct visualization to diagnose adult epiglottitis: a systematic review

\section{Robert Dunfield}

LO74 Does IVC ultrasound independently predict fluid status in spontaneously breathing, undifferentiated hypotensive patients? SHoC-IVC

\section{Ala Bdira}

MP18 Unperceived learning needs among physicians caring for critically ill children in general emergency departments

CIHR-ICRH/CAEP Mid-Career Lecturer Award in Emergency Medicine

\section{Venkatesh Thiruganasambandamoorthy}

Plenary Presentation Prescription for emergency medicine in the era of Dr. Google.

\author{
Abbreviations \\ $\mathrm{PL}=$ Plenary $; \quad \mathrm{LO}=$ Lightning oral $; \quad \mathrm{MP}=$ Moderated poster; \\ $\mathrm{P}=$ Poster
}




\section{Plenary}

\section{PL01}

Rapid agitation control with ketamine in the emergency department: a double blind, randomized controlled trial

D. Barbic, MD, MSc, G. Andolfatto, MD, B. Grunau, MD, MSc, F. Scheuermeyer, MD, MSc, W. Macewan, MD, W. Honer, MD, H. Wong, PhD, S. Barbic, PhD

UBC, Vancouver, BC

Introduction: Rapid control of psychomotor agitation and violent behaviour of emergency department (ED) patients is paramount for patient and staff safety. We conducted a randomized controlled trial comparing intramuscular (IM) ketamine to IM midazolam/haloperidol $(\mathrm{M} / \mathrm{H})$.

Methods: This parallel-arm, double-blind, 1:1 randomized controlled trial compared $5 \mathrm{mg} / \mathrm{kg}$ IM ketamine with $5 \mathrm{mg}$ IM midazolam $/ 5 \mathrm{mg}$ IM haloperidol, with a planned enrolment of 184 patients over 36 months. The primary outcome was the time to adequate sedation, defined as a Richmond Agitation-Sedation Scale (RASS) $<-1$. We used Cox-proportional hazards regression to assess the primary outcome. Secondary outcomes included the proportion of patients experiencing prespecified serious adverse events in each arm.

Results: The trial was terminated early due to a mandate from our institutional REB forbidding non-COVID clinical research. We enrolled 80 patients over 24 months; 54 (68\%) identified as male; median age 35 (IQR 29-41.5); 58 (73\%) arrived via ambulance; 50 $(63 \%)$ had a history of schizophrenia, bipolar disorder or depression; $17(21 \%)$ were HIV positive; and $67(84 \%)$ had a history of illicit substance use. Median time to sedation for MH was 15 min (IQR 1026) versus $6 \mathrm{~min}$ (IQR 4-10) for ketamine. The adjusted Cox proportional hazard ratio favoured ketamine (HR 2.44, 95\% CI 1.44 4.14). Serious adverse events occurred in $2 \mathrm{MH}$ patients and 5 ketamine patients.

Conclusion: For agitated ED patients, ketamine achieves sedation faster when compared to $\mathrm{M} / \mathrm{H}$. The risk of serious adverse events was low in both trial arms. These findings indicate that ketamine is a rapid and safe option for first-line sedation in agitated ED patients. Trial Registration This trial is registered with clinicaltrials.gov (NCT03375671).

Keywords: Agitation; Ketamine; Randomized controlled trial

\section{PL02}

Intracranial hemorrhage after head injury among anticoagulated elderly patients seen in the emergency department: a populationbased cohort study

K. Grewal, MD, MSc, C. Atzema, MD, MSc, P. Austin, PhD, K. de Wit, MD, MSc, S. Sharma, MD, N. Mittmann, PhD, B. Borgundvaag, $\mathrm{MD}, \mathrm{PhD}, \mathrm{S}$. McLeod, $\mathrm{MSc}, \mathrm{PhD}$

University of Toronto/Mount Sinai Hospital, Toronto, ON

Introduction: Elderly patients on anticoagulation are commonly seen in the emergency department (ED). Intracranial hemorrhage (ICH) following head injury is a major concern in this population. The objective of this study was to examine risk of ICH after a head injury among elderly patients anticoagulated with warfarin or a direct oral anticoagulant (DOAC) compared to patients not anticoagulated. Secondary outcomes were 30-day mortality and neurosurgical intervention among patients with ICH.

Methods: Retrospective cohort study using population-based data from 2016 to 2018 of patients $\geq 65$ years who presented to an
Ontario ED with a head injury. Patients were matched 1:1 on the propensity score to create three pairwise-matched cohorts based on anticoagulation status (warfarin vs DOAC, warfarin vs no anticoagulant, DOAC vs no anticoagulant). For each cohort, the relative risk (RR) and 95\% confidence interval (CI) of ICH on the index ED visit was calculated. In patients with ICH, RR of 30-day mortality was calculated for each cohort and Cox proportional-hazards models were used to determine hazard of neurosurgical intervention, accounting for competing risk of death.

Results: There were 77,834 patients with head injury: 64,917 (83.4\%) were not on anticoagulation, $9214(11.8 \%)$ were on DOACs, and $3703(4.8 \%)$ were on warfarin. Overall, $5.8 \%$ of patients had ICH on the index ED visit. There were 3650 pairs $(98.6 \%$ warfarin patients matched) in the warfarin vs DOAC cohort, 3632 pairs $(98.1 \%$ warfarin patients matched) in the warfarin vs no anticoagulation cohort, and 8329 pairs (90.4\% DOAC patients matched) in the DOAC vs no anticoagulation cohort. Patients on warfarin had increased risk of ICH compared to matched patients on DOACs (RR:1.40; 95\% CI 1.181.66) and patients not on anticoagulation (RR:1.42; 95\% CI 1.19 1.69). There was no difference in ICH between those on DOACs compared to matched patients not on anticoagulation. In patients with $\mathrm{ICH}$, there was no difference in 30-day mortality in any matched cohort. Patients on warfarin had an increased hazard of neurosurgery compared to patients on DOACs (HR 1.82; 95\% CI 1.18-2.82) and those not on anticoagulation (HR 1.78; 95\% CI 1.17-2.69). There was no difference in neurosurgery between patients on DOACs versus those not anticoagulated.

Conclusion: Patients on DOACs had less risk of ICH compared to warfarin, and there was no difference in ICH compared to patients not on anticoagulation. Warfarin had an increased risk of ICH compared to those not on anticoagulation.

Keywords: Anticoagulation, Head injury, Intracranial hemorrhage

\section{PL03}

Strategy empowering paramedics to assess low-risk trauma patients with the Canadian c-spine rule and selectively transport them without immobilization: a pragmatic cluster randomized stepped wedge patient-informed multi-center trial

C. Vaillancourt, MD, MSc, M. Charette, MSc, E. Sabri, MSc, E. Hall, B. McLeod, MPH, R. Dionne, MD, J. Prpic, MD, R. Saskin, D. Fergusson, PhD, M. Taljaard, PhD, I. Stiell, MD, MSc

University of Ottawa, Department of Emergency Medicine, Ottawa, $\mathrm{ON}$

Introduction: The Canadian C-Spine Rule (CCR) was previously modified and validated for use by paramedics, then implemented for prehospital safety evaluation in a single-center study. We sought to evaluate a strategy allowing paramedics from multiple communities to assess low-risk trauma patients with the CCR and selectively transport them without immobilization for its effect on patient-determined study outcomes including pain and discomfort.

Methods: This 9-month pragmatic stepped wedge cluster randomized trial took place in 11 Ontario communities. We randomized sites to 1 of 3 sequences (concealed) using covariate constrained allocation, each sequence crossing from the control (usual care) to the intervention (CCR implementation) at 3-month intervals. Paramedics completed a standardized form for all consecutive eligible stable adult patients at risk for c-spine injury. Pain and discomfort scores (10point ordinal scale) were completed when transferring care to emergency staff. We obtained clinical outcomes using linkage to provincial administrative databases and a validated list of ICD-10 codes up to 30 days post injury. Our primary analysis compared immobilization status, pain and discomfort (dichotomized $>4$ 
and $<=4$ ) using random effects logistic regression adjusted for age and sex and accounting for intra-cluster and intra-period correlation, reporting adjusted odds ratios (adjOR) and 95\% confidence intervals $(95 \% \mathrm{CI})$.

Results: In total, 3646 patients were evaluated: mean age 47 years (range 16-103 years), female 51.4\%, MVC $53.2 \%$, fall $30.4 \%$, transported to hospital $93.5 \%$, mean overall pain (4.9/10; SD 3.0) and discomfort (5.6/10; SD 2.9), acute c-spine fracture/dislocation $8.9 \%$, and required stabilization $1.2 \%$. Paramedics chose not to follow the CCR's recommendation to immobilize 4 patients with a significant injury, none sustained a spinal cord injury. There was no difference in total paramedic patient care time between groups. The proportion of patients transported with immobilization significantly decreased from $1334 / 1740(76.7 \%)$ in the control to $813 / 1885(43.1 \%)$ in the intervention periods (adjOR $0.4 ; 95 \%$ CI $0.2-0.6, \mathrm{p}=0.0007$ ). Similarly, pain and discomfort significantly decreased [adjOR for pain: $0.7(95 \%$ CI $0.5-0.9, p=0.009)$ and for discomfort: adjOR $0.6(0.5-0.8$, $\mathrm{p}=0.0007)]$.

Conclusion: In this sizable cluster randomized multi-center trial, paramedics could safely transport a large proportion of low-risk trauma patients without immobilization and much less pain and discomfort.

Keywords: Canadian C-Spine rule, Prehospital, Randomized controlled trial

\section{PL04}

PoCS Rule: derivation and validation of a clinical decision rule for early detection of persistent symptoms after a mild traumatic brain injury

N. Le Sage, MD, PhD, J. Chauny, MD, MSc, S. Berthelot, MD, MSc, P. Archambault, MD, MSc, X. Neveu, MSc, L. Moore, PhD, V. Boucher, MSc, J. Frenette, PhD, E. de Guise, PhD, M. Ouellet, $\mathrm{PhD}$, E. Lang, MD, MSc, J. Lee, MD, MSc, J. Clément, MD, M. Émond, MD, MSc, É. Mercier, MD, MSc, P. Cameron, MD, MSc, J. Perry, MD, MSc

Université Laval, Quebec City, QC

Introduction: The Public Health Agency of Canada recently reported that 5,074,239 Emergency Department visits for head injuries were recorded in the National Ambulatory Care Reporting System between 2002 and 2017, 70.5\% of which were concussions (or mild traumatic brain injury). Although most mild traumatic brain injury patients recover without complications, a wide range of incidence of persistent post-concussion symptoms lasting more than 3 months after their injury is reported in the literature (11-64\%). There are currently no available clinical decision rules that could help emergency physicians to adequately refer patients at risk of persistent symptoms to appropriate post-discharge resources. The main objective of this study is to derive and validate a clinical decision rule for the early prediction of persistent post-concussion symptoms.

Methods: This prospective multicentre derivation and validation cohort study took place in seven Emergency Departments across Canada between 2014 and 2018. Patients aged $\geq 14$ were included if they consulted in the Emergency department following a mild traumatic brain injury that occurred within $24 \mathrm{~h}$. Clinical data were collected in the Emergency Department and symptoms evolution was assessed at 7-, 30- and 90-days post-injury using the Rivermead Postconcussion Questionnaire. The primary outcome was persistent postconcussion symptoms at 90 days after mild traumatic brain injury. A predictive model, called the PoCS Rule, was derived and validated. Results: A total of 1083 patients were included in this study (471 and 612 for the derivation and the validation cohorts, respectively), of whom $15.7 \%$ had persistent post-concussion symptoms at 90 days.
The final model included the following factors in the Emergency Department: age, sex, headache, cervical sprain, haemorrhage on CT, history of traumatic brain injury or psychiatric disorder. The 1-week follow-up allowed the identification of additional risk factors: fatigue, headaches, sleep disturbance, sensitivity to light and Rivermead Postconcussion Questionnaire score $\geq 21$. The PoCS Rule has a $91.4 \%$ and $89.6 \%$ sensitivity, a specificity of $53.8 \%$ and $44.7 \%$ and a negative predictive value of $97.2 \%$ and $95.8 \%$ in the derivation and validation cohorts, respectively.

Conclusion: The PoCS Rule will help emergency physicians to quickly identify mild traumatic brain injury patients at risk of persistent post-concussion symptoms and better refer them to the appropriate post-discharge resources.

Keywords: Decision rule, Mild traumatic brain injury, Post-concussion symptoms

\section{Lightning oral}

\section{LO01}

Practice variation in the management of spontaneous abortion: a multisite retrospective study in Calgary emergency departments

M. Wylie, A. Srajer, K. Lonergan, BSc, P. Brain, BA, MD, E. Lang, MD

University of Calgary, Calgary, $\mathrm{AB}$

Introduction: Practice variation in physician response to spontaneous abortion exists in the Emergency Department (ED). Whereas some physicians may default to consulting the obstetrics-gynaecology (OBGYN) team, increasing the likelihood of a dilation and curettage (D\&C) procedure, others may encourage a non-operative approach to treatment. It remains unclear to what extent spontaneous abortion management in the ED has changed in Calgary over recent years. The primary objective was to determine how the management of spontaneous abortion has changed over time across four Calgary emergency department sites, with emphasis on assessing practice variation between non-operative and operative management. The secondary objective was to assess site-specific differences in operative management, consult and return rates.

Methods: Data were retrospectively collected for patients coded with International Classification of Disease (ICD-10) code O03.4 (incomplete spontaneous abortion without complication) or ICD-10 code O02.1 (missed abortion). Patients with complications or a Canadian Triage and Acuity Scale (CTAS) score of 1 were excluded $(n=3845)$. Return to care for future D\&C was considered a proxy for failed non-operative management. Data were analyzed using Chisquare and Mann-Whitney U tests.

Results: Of the included patients, 1110 (28.9\%) received operative management via D\&C. The remaining $2735(71.1 \%)$ patients received medical or expectant management. The site of presentation influenced $\mathrm{D} \& \mathrm{C}$ rates (Site $1=33.5 \%>$ Site $2=29.5 \%>$ Site $3=28.7 \%>$ Site $4=15.8 \%, p<0.001)$. The rate of D\&Cs decreased from $34.2 \%$ in 2014 to $22.6 \%$ in 2019 across all sites $(\mathrm{p}<0.001)$. Site 4 is unique in that ED staff redirect stable patients presenting with spontaneous abortion to an early pregnancy loss clinic. There was minimal variation in rates of ED returns and returns resulting in D\&Cs within 7 days. Patients who underwent $\mathrm{D} \& \mathrm{C}$ were older $(\mathrm{p}=0.009)$, had lower hemoglobin $(\mathrm{p}<0.001)$, shorter triageto-assessment times $(\mathrm{p}<0.001)$, lower CTAS scores $(\mathrm{p}<0.001)$, and spent more time in the ED $(\mathrm{p}<0.001)$.

Conclusion: The management of spontaneous abortion in the ED has shifted over time towards non-operative management. This shift may be driven by models of care that redirect spontaneous abortion from 
the ED and the practice patterns of OBGYN and ED physicians. Reductions in D\&Cs were not associated with an increase in ED revisits, supporting the effectiveness and safety of non-operative management.

Keywords: Dilatation and curettage, Management variation, Spontaneous abortion

\section{LO02}

Managing children with acute bronchiolitis: a systematic review
and network meta-analysis

S. Elliott, PhD, L. Gaudet, MSc, R. Fernandes, MD, PhD, B. Vandermeer, MSc, S. Freedman, MD CM, MSc, D. Johnson, MD, A. Plint, MD, T. Klassen, MD, D. Allain, MD, L. Hartling, PhD

University of Alberta, Edmonton, $\mathrm{AB}$

Introduction: In Canada over 35/1000 children under 1 year of age are hospitalized annually with bronchiolitis, with estimated yearly costs of over $\$ 23$ million. Uncertainty exists as to which treatments are effective. We conducted a systematic review and network metaanalysis (NMA) to evaluate the comparative effectiveness of the most commonly used treatments for managing bronchiolitis among children less than 2 years.

Methods: We included six interventions: bronchodilators, glucocorticoid steroids, hypertonic saline solution, antibiotics, heliox therapy and high flow oxygen therapy. Search results from four primary literature databases, two clinical trial registries and relevant conference proceedings were screened based on a priori inclusion criteria. Data were extracted and independently verified. Cochrane Risk of Bias tool was used to assess risk of bias in individual studies. Primary outcomes were admission rates (ARs) and hospital length of stay (LOS). Frequentist NMA estimated mean differences (MD) with $95 \%$ confidence intervals (CI) of each treatment compared to nebulised placebo. Treatment effectiveness was ranked based on Surface Under the Cumulative Ranking curve (SUCRA).

Results: 150 studies were included; 27 studies (4656 patients) reported relevant data for ARs while 57 studies (7605 patients) provided data for LOS. For ARs, nebulized epinephrine (OR 0.64, 95\% CI 0.44-0.93) and nebulized hypertonic saline plus salbutamol (OR $0.44,95 \%$ CI $0.23-0.84)$ were both more effective than nebulized placebo (i.e., $0.9 \%$ saline); no other treatments significantly affected ARs. Hypertonic saline plus salbutamol was ranked as the most effective treatment, and was significantly more effective than nebulized salbutamol alone (OR $0.55,95 \%$ CI $0.33-0.91$ ). For LOS, nebulized hypertonic saline (MD $-0.64,95 \% \mathrm{CI}-1.01$ to -0.26 ) and nebulized hypertonic saline plus epinephrine (MD - 0.91, 95\% CI -1.14 to -0.40 ) were effective compared to nebulized placebo (i.e., nebulised $0.9 \%$ saline). Nebulized hypertonic saline plus epinephrine was the top ranked treatment.

Conclusion: Evidence shows the effectiveness and superiority of hypertonic saline with salbutamol to reduce ARs. Nebulized hypertonic saline alone, or in combination with epinephrine, seems beneficial to reduce LOS. Understanding the most appropriate management for bronchiolitis has the potential to mitigate inappropriate healthcare utilization, reduce costs, and improve outcomes.

Keywords: Bronchiolitis, Children, Network meta-analysis

\section{LO03}

The utility of telemedicine in pediatric emergency care: a scoping review

O. Robinson, BSc, S. Zouboules, BSc, H. Lafave, BSc, R. Galbraith, MD, N. Dunnewold, MLIS, E. Lang, MD
Foothills Medical Center, Calgary, AB

Introduction: Public health measures to combat COVID-19 and escalated parental fear in seeking medical care have compromised pediatric access to acute care, leading providers to explore alternate delivery models such as telemedicine. As of March 2020, pediatric emergency departments (EDs) continue to see marked reductions in visits without evidence of a reduction in needs. While pediatric emergency telemedicine (PET) has the potential to lessen gaps in care, it is critical that implementation is rooted in evidence to ensure patient safety and efficacy. This study aims to map the existing literature and identify research gaps pertaining to the use of telemedicine in pediatric emergency care.

Methods: A scoping review was conducted per the PRISMA-ScR checklist. Three databases (MEDLINE, EMBASE, CINAHL) were searched on Sept 15, 2020 to retrieve publications from 2010 on.

Results: Searches of the databases returned 1617 studies. Following two-step review, 37 studies were included. The most common study design was observational $(\mathrm{N}=23)$, followed by descriptive $(\mathrm{N}=14)$, review $(\mathrm{N}=2)$, experimental $(\mathrm{N}=1)$ and quality improvement $(\mathrm{N}=1)$. Studies were categorized based on their primary application and impact of telemedicine (positive, negative and neutral) including direct-to-consumer telemedicine $(\mathrm{N}=6$; 3pos, 2neg, 1neut), rural applications $(\mathrm{N}=11 ; 7$ pos, 1neg, 3neut), general ED utility $(\mathrm{N}=9$; 6pos, 1neg, 2neut), transports ( $\mathrm{N}=7$; 4pos, 3neut) and sub-specialist teleconsultation $(\mathrm{N}=4$; 4pos). The number of studies reporting data on the outcomes of interest were as follows: prevalence $(\mathrm{N}=7)$; current applications $(\mathrm{N}=16)$; patient outcomes $(\mathrm{N}=21)$; patient satisfaction $(\mathrm{N}=6)$; provider satisfaction $(\mathrm{N}=13)$; feasibility, challenges and barriers $(\mathrm{N}=20)$. The majority of studies demonstrated positive conclusions favoring telemedicine utility $(\mathrm{N}=24)$, while four had largely negative conclusions (for reasons of low demand, decreased antibiotic stewardship or workflow challenges). The remainder were neutral $(\mathrm{N}=9)$.

Conclusion: PET programs hold promise in facilitating access to care, while minimizing the health risks associated with visiting the ED during the COVID-19 pandemic. Although publications are emerging, the body of literature remains scarce with majority of publications being observational or descriptive. Further research is needed to better characterize the clinical utility and safety profile of PET and to determine specific strategies to overcome barriers to use. Keywords: Emergency, Pediatric, Telemedicine

\section{LO04}

Predicting pediatric hospital admission at emergency department triage: a derivation and internal validation study using retrospective data from a tertiary care hospital in Northwestern Ontario

\section{S. Stanojev, BSc, MD}

Northern Ontario School of Medicine, Thunder Bay, ON

Introduction: The early determination of patient disposition in the Emergency Department (ED) is a critical aspect of clinical decision making that determines department flow and downstream resource allocation. The objective of this study was to derive and internally validate a predictive model for pediatric admission from the ED in a tertiary care hospital.

Methods: This retrospective study analyzed all ED triage data of pediatric (age $<18$ years) presentations to Thunder Bay Regional Health Sciences Centre (TBRHSC) over a 1-year period. The outcome of interest was patient admission to hospital including patient transfer to quaternary care centres. Google BigQuery and Microsoft 
Excel were used for data analysis including machine learning. Logistic regression derivation models were developed using patients' triage data including patient age, sex, mode of arrival, time of arrival, CTAS and chief complaint. Patient presentations were randomized to either the model derivation group (80\%) or validation group $(20 \%)$. Results: The TBRHSC ED dataset included 15980 pediatric presentations that took place between May 2016 and April 2017. Of these $51.6 \%$ were male and the mean (SD) age was 6.4 years (4.9) with an overall admission rate of $5.5 \%$. The final multivariate regression model had an area under the receiver operating curve of 0.89 with a $\log \operatorname{loss}$ of 0.32 . A probability cut-off was selected as the intersection of optimal test characteristics and produced a sensitivity of $43.4 \%$ and specificity of $97.3 \%$ with an odds ratio of 27.7 (95\% confidence interval $19.3,40.0$ ).

Conclusion: Using solely pediatric demographic data available at triage, a logistic regression derivation model was produced that identifies patients at high probability of admission even before physician assessment. Given the model's current low sensitivity, however, there would be a relatively large proportion of potential admissions missed in this prediction. Next steps would involve improving the model's predictive strength through inclusion of additional patient factors available early in the patients' visit (for example vital signs, bloodwork or imaging tests). Such a model could be integrated into an ED's EMR to predict hospital admission from the ED many hours before the clinical decision to admit has been formally made. This would allow for improved resource utilization including bed allocation, thereby improving patient flow and decreasing unnecessary boarding time in the ED.

Keywords: Decision, Risk score, Triage

\section{LO05}

\section{A longitudinal, population-based study on mental health outcomes in child survivors of traumatic injury compared with matched controls}

D. Chung, MD, S. Logsetty, MD, J. Gawaziuk, MSc, N. Cristall, PhD

University of Toronto/St. Joseph's Health Center, Toronto, ON

Introduction: Pediatric traumatic injury (PTI) is a common presentation in the emergency department and the leading cause of hospitalization among children. Little is known about subsequent mental health outcomes after traumatic injury in the pediatric population. This longitudinal cohort study compared the mental health outcomes in survivors of PTIs with matched controls from the general population. This study hypothesized that survivors of PTIs will have increased rates of mental health outcomes relative to matched controls.

Methods: Children under 18 years old with an injury requiring hospitalization at a regional pediatric trauma center between January 1, 2004 to December 31, 2016 ( $\mathrm{n}=9551)$ were matched to five controls from the general population $(n=47,755)$. Individuals were matched based on age, sex, income, and geographic region. Mental health outcomes that were diagnosed within 2 years of the injury were captured through physician billing and hospitalizations, dating to December 31, 2018. Pre- and post-injury adjusted relative rates (ARRs) of anxiety, depression, and substance use disorders were calculated with generalized estimating equations. ARRs were compared and a group-period interaction term was used to determine the significance of the change over time.

Results: Survivors of PTI had increased ARRs of anxiety (1.66, 95\% CI 1.51-1.82); depression (2.87, CI 2.57-3.21); substance use RR = 3.23, (CI 2.64-3.99); and any mental health outcome (1.90, CI 1.762.04 ). They also had high pre-injury rates: (anxiety 1.30 , CI $1.16-$ 1.47); depression (2.00, CI 1.73-2.32); substance use (4.99, CI 3.08-
5.20) and any mental health outcome (1.50, CI 1.37-1.66). Comparing the pre- and post-injury ARRs showed a significant difference over time $(\mathrm{p}<0.001)$ for anxiety, depression, and any mental health outcome. There was no significant difference in substance use over time. Conclusion: Child survivors of PTIs had increased ARRs of anxiety, depression, and any mental health outcome compared to matched controls. These findings highlight the need to revise injury prevention recommendations, target intervention strategies in the management of PTIs, and the importance of a trauma informed approach to care, specifically in the pediatric population.

Keywords: Pediatrics, Population level study, Trauma

\section{LO06}

Pet therapy in the emergency department and ambulatory care: a systematic review

S. Ali, MD CM, S. Elliott, PhD, L. Gaudet, MSc, E. Reiter, PhD, L. Dennett, MLIS, S. Scott, BN, PhD, B. Felkar, MSc, B. Stauffer, $\mathrm{MD}$, L. Hartling, PhD

University of Alberta, Edmonton, AB

Introduction: Pet therapy, or animal-assisted interventions (AAI), have been demonstrated to have a positive effect for patients, families and health care providers (HCP) in inpatient settings. However, the evidence supporting AAI in emergency or ambulatory care settings is less clear. We conducted a systematic review to evaluate the effectiveness of AAI on patient, family, and HCP experience in these settings.

Methods: We searched (from inception to May 2020) six databases (Medline, Embase, Cochrane CENTRAL, PsycINFO, and CINAHL) and grey literature for studies assessing AAI in emergency and ambulatory care settings. Our primary outcomes were: (1) patient and family distress/anxiety or pain; and (2) HCP stress, satisfaction, or well-being. Screening, data extraction and quality assessment were done in duplicate with conflicts resolved by third party adjudication. Random-effects meta-analysis was completed in RevMan5.3 for outcomes reported by $\geq 3$ studies and are reported as mean differences (MD) or standardized mean differences (SMDs) and 95\% confidence intervals (CIs), as appropriate. Risk of bias (ROB) was assessed using Cochrane's Risk of Bias 2.0 tool, which assesses ROB for individual outcomes.

Results: We identified 3057 records and included 14 studies: 4 before/after, 1 mixed-methods and 9 randomized controlled trials (RCTs). Only results from RCTs are reported here. RCTs were carried out in the ED $(n=3)$, outpatient clinics $(n=5)$, or a dental clinic $(\mathrm{n}=1) ; 5$ care settings were pediatric. Most AAIs $(8 / 9,89 \%)$ utilized trained therapy dogs. We found no evidence of effectiveness for AAIs in improving observed patient anxiety/distress (4 RCTs; $n=166$; SMD $-0.48,95 \% \mathrm{CI}-1.10$ to 0.13 ); self-reported patient anxiety/ distress ( 3 RCTs, $\mathrm{n}=380$; SMD $-0.36,95 \% \mathrm{CI}-0.95$ to 0.23 ); or self-reported pain ( 3 RCTs, $\mathrm{n}=202$; $\mathrm{MD}-1.12,95 \% \mathrm{CI}-2.65$ to 0.40). Two RCTs reported no effect on parental anxiety. Two RCTs reported reduced stress in HCPs after AAI. ROB for all comparisons of observed anxiety/distress outcomes was rated as "some concerns". For all comparisons of self-reported anxiety ROB was rated as "high". For comparisons of self-reported pain, ROB was rated as "high or "some concerns".

Conclusion: Limited evidence (due to small sample sizes and high ROB) is available on the effectiveness of AAI for families in emergency and ambulatory care settings. More robust and rigorous studies are required to strengthen the evidence base for use of AAI to improve patient, family and HCP experience.

Keywords: Patient experience, Pet therapy, Systematic review 


\section{LO07}

\section{Identification of pediatric emergency department patients for referral to rapid-access substance use services}

E. Williams, MD, M. Koziak, BSc, J. Hann, MD, A. Gauri, MSPH, S. Ali, MD CM, A. Kirkham, MD

University of Alberta, Edmonton, $\mathrm{AB}$

Introduction: Pediatric emergency department (ED) visits related to substance use are rising. Such patients are often discharged from the ED without adequately addressing their underlying substance use, which contributes to repeat ED visits and increased substance-related harms. Rapid access addiction clinics have been an effective model of care in adults. Providing similar services for the pediatric population could positively influence their substance use trajectory into adulthood. Our study objective was to assess reported need for rapidaccess substance use services for pediatric patients presenting with substance-related ED concerns.

Methods: Prospective patient enrolment was conducted over a 10-week period from May to August 2019 at the Stollery Children's Hospital ED in Edmonton, Alberta. Patients $<18$ years with known or suspected substance use disorders were enrolled by various members of the ED health care team using a one-page enrolment form. Electronic and paper records from the ED index visit were reviewed by the research team. The primary outcome was whether the ED team would have referred the patient to an on-site rapid-access addiction clinic, if this service was available. Other outcomes such as patterns of substance use and ED utilization were also evaluated.

Results: We enrolled 119 patients; 100 patients were included in final analysis. Sixty-one percent of included patients were female and the median age was 15 years. The most commonly reported substances used were alcohol (49\%), cannabis (40\%), and stimulants (28\%). In the 30 days prior to the index ED visit, 34 patients had at least one prior ED visit. Health care team members identified 57 patients as appropriate for referral to rapid-access substance use services. The most common reasons why patients were not referred were: perception that the patient would not accept the referral (33\%), the patient had follow-up arranged elsewhere (19\%), and the patient did not live in the city $(12 \%)$.

Conclusion: The ED is often a first point of contact for many patients, making it imperative to connect patients to substance assessment and intervention at the time of ED visit. Over a 10-week period, 57 pediatric patients with substance use-related presentations were identified by their healthcare providers as appropriate for referral to rapid-access substance use services. This study suggests an unmet need for rapid-access substance use services for pediatric ED patients. Further exploration into development of the service is warranted.

Keywords: Pediatric emergency medicine, Referral and consultation, Substance-related disorders

\section{LO08}

\section{Adolescent and caregiver perspectives on living with a limb fracture}

S. Golden-Plotnik, BA, MD, BScMed, M. Bharadia, BSc, M. Van Manen, MD, PhD, M. Sivakumar, MN, BScN, A. Drendel, MSc, DO, N. Poonai, MD, MSc, M. Moir, BN, MSc, S. Ali, BSc, MD CM

University of Alberta, Edmonton, $\mathrm{AB}$

Introduction: Fractures occur in up to half of children before the age of 16 years. After initial emergency care for a fracture, function is universally impaired in children, and impacts extend to the family. Our study objectives were to understand: (1) how adolescents (1217 years) describe the functional impact of fractures on their lives, (2) how adolescents' reports of their experience compare to those of their caregivers, and (3) the impact of the fracture on the family unit.

Methods: We conducted individual, semi-structured interviews with adolescents and their caregivers 7-14 days following their initial visit to the Stollery Children's Hospital emergency department (Edmonton, Alberta). We utilized qualitative content analysis methodology; recruitment proceeded from June 2019 to November 2020, until thematic saturation was reached. Coding and analysis were concurrent with recruitment and interviews. A secondary coder reviewed $40 \%$ of the transcripts, and the coding team met regularly to discuss the coding scheme and key themes. The interview script was modified in an iterative process, to reflect emerging themes.

Results: We completed a total of 29 interviews. The most commonly affected functions were: (a) showering and hygiene (which required the most caregiver support), (b) sleep variability (due to pain and castrelated discomfort), and (c) exclusion from sports/activities. Many adolescents experienced disruptions to social activities and gatherings. Adolescents valued independence and often chose to take more time to complete a task to preserve this, regardless of inconvenience. Both adolescents and caregivers reported feelings of frustration and loneliness. Generally, caregivers' perspectives were in keeping with the experiences that adolescents described for themselves. Family impacts included conflicts that arose when a sibling took on extra chores/tasks.

Conclusion: These themes highlight an opportunity to better tailor emergency discharge instructions for adolescents with fractures. Key messages include: (1) expect pain and impaired sleep especially in the first few days, (2) allow extra time to complete tasks independently, especially for grooming and hygiene, (3) consider family impact, especially siblings, (4) prepare for changes in activities and social dynamics, and (5) frustration is normal, for both adolescents and caregivers. Future work can focus on developing a novel, familyinformed tool to assess functional outcomes following injury.

Keywords: Children, Fractures, Functional outcomes

\section{LO09}

What we know about acute pediatric cannabis poisoning: a scoping review

L. Gaudet, MSc, S. Elliott, PhD, K. Hogue, MD, S. Scott, PhD, RN, L. Hartling, $\mathrm{PhD}$

University of Alberta, Edmonton, AB

Introduction: Liberalization of cannabis policy in Canada has led to concerns that increasing access to cannabis and cannabis infused products (e.g. edibles) for adults, will have negative, unintended consequences for children. We conducted a scoping review to map the available evidence on the public health implications, clinical management, and experiences and information needs of healthcare providers (HCP) and parents, relating to acute cannabis poising in children.

Methods: We searched three databases (Medline, CINAHL, and PsychINFO) from inception until October 2019. Grey literature was searched up to March 2020. We included all records (primary research, knowledge synthesis, editorials, letters/opinion pieces, government websites and news articles) reported in English or French. We defined acute cannabis poisoning as unintended exposure to cannabis or cannabis product(s) that led to the need for acute medical advice or treatment. A primary reviewer screened all identified publications and a secondary reviewer screened excludes. Records were categorised by area (public health implications, clinical 
management, experiences and information needs) and mapped based on reported outcomes and record type.

Results: We identified 4644 records and included 158 [knowledge syntheses $(n=3)$; primary research $(n=95)$; editorials, commentaries, and letters $(\mathrm{n}=18)$; education or media for $\mathrm{HCP}(\mathrm{n}=15)$; and education or media for the public $(n=27)$ ]. Public health implications were addressed by $129(82 \%)$, often reporting increasing incidence of acute paediatric cannabis poisonings. 116 records (51 [44\%] case studies) reported or discussed clinical characteristics and/ or management of acute paediatric cannabis poisoning, including signs and symptoms $(92 \%)$, use of toxicology screening (60\%), type of clinical management $(52 \%)$ and treatment recommendations $(36 \%)$. Few sources addressed either HCP $(n=5)$ or parent $(n=1)$ experiences or information needs.

Conclusion: The increasing incidence of acute cannabis poisonings and liberalization of cannabis legislation is clear, however, evidence around the clinical management is limited. Rigorous studies of suggested clinical treatments are needed to develop an evidence-base from which data can be synthesized and appraised, in order to drive the development of clinical recommendations. Additionally, further research exploring $\mathrm{HCP}$ and parent experiences and information needs around cannabis poisoning is warranted.

Keywords: Cannabis, Poisoning, Scoping review

\section{LO10}

\section{Implementation of trauma team activation criteria: a quality improvement project to reduce overtriage at a level 1 trauma centre}

A. Verma, MD, MHSc, B. Tillmann, MD, K. Pardhan, MD, MMed, A. Nathens, MD, PhD, W. Thomas-Boaz, MN, BScN, C. Freedman, MHI, L. Notario, MSc, A. Phillips, BA, L. Da Luz, MD, MSc

\section{Sunnybrook Health Sciences Centre, Toronto, ON}

Background: Severely injured trauma patients have better outcomes when treated in trauma centres with a dedicated Trauma Team Activation (TTA). However, TTA is resource intensive, and must be used judiciously. Overtriage, defined as a TTA that does not meet criteria for severe injury after retrospective review, happens frequently at our centre. Historically, we used the Emergency Medical Services field triage criteria with or without input from Emergency Department (ED) physicians or nurses to activate the trauma team. On May 1, 2020, we implemented a formal process and evidence-based criteria to determine if TTA was warranted.We sought to reduce overtriage from 50 to $35 \%$ within 3 months of implementation of the new TTA process and criteria.

Aim statement: ED triage nurses were provided with the new TTA criteria and algorithm, authorizing them to initiate the TTA when the criteria were met. If there was uncertainty, they were instructed to call an ED physician to assess using a checklist to help determine if TTA was warranted. The primary outcome measure was the proportion of overtriage out of all TTAs (excluding transfers). This was evaluated using a run chart, with overtriage rates calculated weekly for 3 months before and after implementation. A process measure was the number of patients assessed by ED physicians for possible TTA, and the proportion that led to TTA. A balancing measure was undertriage, defined as the proportion of patients assessed by ED physicians who did not have a TTA and did meet severe injury criteria.

Measures and design: Fewer patients were overtriaged during the 3 months after implementation $(144 / 316 ; 46 \%)$ than before implementation $(153 / 287 ; 53 \%)$. There were 94 patients assessed by ED physicians for possible TTA, of which 27 (29\%) proceeded to TTA. Out of all 94 patients, upon retrospective review 2 (2\%) were undertriaged. We reviewed both cases and modified our criteria after one. The trauma team was activated later for one of the cases and no negative outcomes resulted from the undertriage in either case.

Evaluation/results: The implementation of the process and criteria reduced overtriage but did not achieve our target. However, an absolute reduction in overtriage of $7 \%$ translated to 23 fewer TTA in 3 months, which is important. We are continuing PDSA cycles to further refine the criteria. We believe these criteria could be implemented at other trauma centres seeking to reduce their overtriage rate. Keywords: Overtriage, Quality improvement and patient safety, Trauma

\section{LO11}

A quality improvement initiative to provide COVID-19 patients with rapid follow up via a novel multi-disciplinary virtual clinic following an emergency department visit

S. Masood, MD, MPH, A. Boggild, MD, MSc, Y. Moayedi, MD, A. Sidhu, MD, N. Marks, MN, S. Alsenchuk, MN, J. Locquiao, BSc, T. Carlyle, M. Harper, M. Chang, BA, S. Isaackz, MHSc, R. Wu, MD, MSc, J. Granton, MD

\section{University Health Network, Toronto, ON}

Background: The COVID-19 pandemic has resulted in a significant strain on emergency departments (ED) and hospitals globally, and highlighted the challenges associated with direct in-person care due to the risk of COVID-19 transmission. Specifically, this risk is highest when assessing patients diagnosed with COVID-19. As a result, many COVID-19 patients are left without follow up after diagnosis resulting in frequent ED visits for COVID-19 related symptoms. Our goal was to provide $95 \%$ of ED patients with follow up within $48 \mathrm{~h}$ of diagnosis by 3 months of launch of our virtual clinic.

Aim statement: We used the IHI's model for improvement and the gemba framework to rapidly develop and implement our initiative. We engaged in frequent and regular stakeholder analyses to develop our driver diagram and Plan-Do-Study-Act (PDSA) interventions. We developed a virtual multi-disciplinary clinic that included ED and sub-speciality physicians, nurse practitioners and social workers to provide ED patients with rapid and comprehensive follow up. Our PDSAs included (1) development of an electronic referral and provider reminders to refer patients. (2) Simplification of documentation using a streamlined webform. (3) Automation of the referral process through electronic health-record system triggers. (4) Remote monitoring of high-risk patients through home $\mathrm{O}_{2}$ monitoring. Our primary outcome measure was the no. of ED patients diagnosed with COVID seen in the clinic. Process measures included the number of ED referrals to the clinic. Balancing measures were ED return visits within 1 week of initial visit.

Measures and design: A total of 300 patients were referred from the ED in the first 6 months of the program. $100 \%$ of patients were seen within $24 \mathrm{~h}$ of being referred from our ED by 3 months. 14 (4.6\%) of patients returned to an ED within 1 week of their index ED visit, of which $5(35.7 \%)$ patients required readmission.

Evaluation/results: Our multi-faceted QI initiative provides ED patients with COVID-19 safe, rapid and longitudinal follow up. Our ED re-visit rates are lower than those previously reported in the literature, however readmission rates are consistent with published data, suggesting that our initiative was successful in preventing unnecessary ED visits, while facilitating appropriate admissions to hospital for care escalation. It provides a scalable and sustainable model that can be adapted at other institutions during the current pandemic.

Keywords: COVID-19, Quality improvement and patient safety, Remote monitoring 


\section{LO12}

A quality improvement project to enhance emergency department intubation performance and decrease complications during the COVID-19 pandemic

J. Yoo, MD, S. Wong, BSc, K. Dullemond, MD, C. Renschler, MD

University of British Columbia/St. Paul's Hospital, Vancouver, BC

Background: The COVID-19 pandemic has forced emergency departments (EDs) to change endotracheal intubation (ETI) protocols because of the risk of disease transmission to health care providers. ETI is regularly performed in the ED and first-attempt success (FAS) is associated with better patient outcomes. Our setting is a tertiarycare ED in Vancouver, BC, with an average of 170 ETIs performed annually. Prior to the pandemic, FAS and complication rates in our ED were below the standard-of-care of $83 \%$ and $13 \%$, respectively. To increase FAS above $83 \%$ and decrease complication rates below $13 \%$ in our ED by April 1, 2021.

Aim statement: This quality improvement (QI) study was conducted over a 20 month period. A pre-intervention cohort of ETIs performed over 12 months prior to the COVID-19 pandemic (Mar 1, 2019-Feb 29, 2020) was used to identify practice patterns and to design QI interventions, including a sequential ETI checklist, standardized personal protective equipment (PPE), PPE donning and doffing posters, equipment and medication kits, and the departmental distribution of an ETI training video illustrating proper protocol use. Interventions were implemented using PDSA cycles and iterative learning. Standardized registry data from every ETI performed in the ED was used to monitor the impact of interventions during an 8 month post-intervention period. Monthly run charts were used to illustrate the impact of interventions and to assess sustainability of change. Potential harms from study interventions were monitored.

Measures and design: Pre-intervention, 140 ETIs were performed compared to 90 in the post-intervention period. FAS increased from 80.7 to $86.7 \%$. Complication frequency decreased from 15.7 to $11.1 \%$. Relative risk reduction using pandemic protocols was $70.7 \%$ with a number needed to treat of 21.7. Monthly FAS remained above the stated goal of $83 \%$ and monthly complication rates remained below the pre-intervention median of $16.0 \%$ for the last 6 months of the study, indicating shifts for both outcomes. No significant harm from study interventions was identified.

Evaluation/results: We describe a QI project that used pandemic protocols to improve team performance and patient safety during ETI. Sustained shifts in ETI performance and complication rates were observed 2 months after intervention and aims were achieved before the stated goal date. Simple QI interventions were used in this study that can be applied to other EDs and critical-care settings.

Keywords: Coronavirus, Intubation, Quality improvement and patient safety

\section{LO13}

Improving safety and communication for healthcare providers caring for SARS-COV-2 patients

A. Taher, MD, MPH, P. Glazer, MD, C. Culligan, MD, S. Crump, MScN, S. Guirguis, MA, J. Jones, PhD, A. Dharamsi, MD, L. Chartier, MD CM, MPH

University of Toronto, Toronto, ON

Background: Over 300,000 healthcare providers (HCPs) have contracted the severe acute respiratory syndrome coronavirus 2 (SARSCOV-2) worldwide, with emergency departments (EDs) being high- risk settings. Approaches to decrease exposure include sealing isolation rooms and limiting HCP presence in them, both of which can result in communication difficulties. This quality improvement (QI) initiative aimed to decrease by $50 \%$ the length of time isolation room doors stayed open, and to increase HCP-perceived communication clarity by one point on a five-point Likert scale, over a three-month period.

Aim statement: This prospective, multi-stage project had three PlanDo-Study-Act (PDSA) cycles between May and July 2020: (1) an educational intervention to ensure awareness; (2) the introduction of a novel device termed "transceiver" to improve communication across closed doors; and (3) local provider engagement through a clinical champion, who was introduced as a result of stakeholder feedback. Project measures included: primary outcome-breaches in isolation room doors; secondary outcome-HCP-perceived communication clarity; process-percentage patients meeting isolation criteria; balancing-HCP-perceived communication errors, use of workarounds, and HCP workflow interruptions. Statistical Process Control XbarR charts assessed special cause variation, and two-tailed Mann-Whitney $\mathrm{U}$ tests were used for statistical significance between means for Likert scale surveys.

Measures and design: Observation of 174 patient encounters (range 5-95 min) was completed over 33 days, with 95 meeting inclusion criteria. Door opening decreased from baseline $(n=40$; mean $72.97 \%)$ to PDSA $3(\mathrm{n}=21$; mean $1.58 \%$; $\mathrm{p}<0.0001)$. SPC charts showed special cause variation during the baseline period, accounted for by a local system change (adding curtains affecting door opening), and an improvement was noted by PDSA-2 and 3 with no further special cause variation. HCP-perceived communication clarity improved from baseline $(n=36$; mean 3.36$)$ to PDSA-3 $(n=49$; mean $4.21 ; \mathrm{p}<0.001)$. Percentage of patients meeting isolation criteria (process) showed a slow decline. Balancing measures showed significant improvements.

Evaluation/results: Our iterative QI approach with education, a novel technology, and a local champion demonstrated significant improvements in door breeches and communication clarity. Future work will further improve workflow integration and may expand to other areas such as operating rooms and intensive care units.

Keywords: Emergency department, Quality improvement and patient safety, SARS-COV-2

\section{LO14}

Reducing hospital admissions using an interprofessional team in an academic emergency department

A. Verma, MD, MHSc, N. Coyle, MSc, W. Thomas-Boaz, MN, BScN, K. Pardhan, MD, MMed, S. Michaelson, BA, BScN, MHS, N. Ziegler, MSW, E. Elliot, MSW, B. Ciacco, MSW,

S. Shadowitz, MD CM, MSc, J. Stanway, MSc, L. Fillion, BScN, MHSc, A. Smith, MD, MSc, I. Cheng, MD, MSc, PhD

\section{Sunnybrook Health Sciences Centre, Toronto, ON}

Background: Sunnybrook Emergency Department (ED) has higher admission rates compared to peer hospitals, contributing to ED crowding. This rate was higher during evening hours, when health professionals such as the geriatric nurse and social worker were not available. According to a root cause analysis, a major contributing factor to high admission rates was the lack of these health professionals and community integration. In Nov 2019, we implemented the "ED One Team" (ED1T), an interprofessional team focused on facilitating safe ED discharges with community integration, available every day from 8AM to 11PM.Within 5 months of ED1T implementation, we aimed to reduce the number of CTAS 2-5 ED patients admitted to hospital by 3 per day. 
Aim statement: The primary outcome measure was the change in the number of admissions, compared to the same time period the year before ED1T. We performed a subgroup analysis for senior patients $\geq 70$ years. The process measure was the number of patients seen and discharged by ED1T. The balancing measure was the 7-day ED revisit rate for senior patients seen by ED1T compared to those not seen by ED1T.

Measures and design: From Nov 1, 2019 to Mar 31, 2020, there were 28.6 admits/day (admit rate 19.6\%). Before ED1T, there were 30.8 admits/day (19.8\%). For seniors, there were 15.4 admits/day (35.0\%) compared to 14.0 admits/day (35.7\%) before ED1T. Admissions dropped by 2.2/day (1.4/day for seniors). The ED1T saw 987 patients in the 5 months ( 6.5 patients/day) with a discharge rate of $68.3 \%$. The revisit rate for seniors was $5.9 \%$ for patients seen versus $7.1 \%$ for those not seen by ED1T.

Evaluation/results: We reduced our admission number and rate but fell short of target. We continue to make changes via PDSA cycles to optimize the success of the ED1T. In the 5-month period, we reduced the number of admissions by 330. Assuming an average hospital length-of-stay of 5 days, 1650 potential bed-days were saved. There was not an increase in the revisit rate in those patients who were discharged by the ED1T. Emergency Departments should consider adding similar interprofessional teams to decrease hospital admissions.

Keywords: Health professionals, Patient flow, Quality improvement and patient safety

\section{LO15}

\section{Decreasing invasive urinary tract infection screening} in a paediatric emergency department: a quality improvement initiative

F. Paluck, MD, I. Kestenbom, MD, G. Test, MD, B. Brimmer, BScN, O. Ostrow, MD

University of Toronto, Toronto, ON

Background: Fever is a common presentation to the Emergency Department (ED) among children and a urinary tract infection (UTI) often needs to be excluded. Sterile techniques, like catheterization, are invasive, can be traumatizing to children, and are time consuming to complete. A two-step approach has been shown to reduce the catheterization rate in febrile, young children without unintended consequences. Our aim was to implement a two-step approach for UTI screening in febrile children 6-24 months in order to decrease the number of unnecessary urine catheterizations by $50 \%$ without impacting ED length of stay (LOS), return visits (RVs) or missed UTI diagnoses.

Aim statement: After engaging key stakeholders and a nursing champion, we created a process map to understand the current urine collection process in our ED, and areas for targeted improvement. Using the model for improvement, we designed a 2-step pathway for a suspected UTI in children 6-24 months as our change idea. The pathway involved identifying children who met inclusion criteria for UTI screening, followed by urine bag application and urinalysis (UA) if clinically indicated. If UA was positive, a second urine sample was collected via catheterization, for repeat UA and culture. Through multiple PDSA cycles, our pathway was implemented in the ED along with concurrent staff education. The outcome measure was the rate of ED urine catheterizations. Process measures included the total number of urine cultures sent to microbiology and percent positivity. The balancing measures included ED LOS and RVs.

Measures and design: Since project initiation in July 2019, the ED catheterization rate decreased from 73 to $53 \%$ and the number of urine cultures sent to Microbiology decreased by $23 \%$. The average number of monthly catheters completed decreased from 126 with a positivity rate of $17 \%$, to 96 with positivity rate of $19 \%$. There was no significant change in ED LOS or RVs over the 15 month study period. Evaluation/results: Using improvement methodology, we successfully decreased the number of unnecessary catheterizations and urine cultures sent to microbiology, without unintended consequences. To achieve our aim, further refinements to our intervention are ongoing and include optimizing urine screening equipment in patient rooms, poster reminders, re-education for providers, and introducing a parent resource explaining the 2-step pathway. There are also plans to spread this initiative to the inpatient pediatric units.

Keywords: Paediatric urinary tract infection, Quality improvement and patient safety, Urine bag collection

\section{LO16}

Improving testing for sexually transmitted infections at St. Michael's Hospital emergency department in Toronto

S. Gupta, MD, E. Austin, MD, M. Bignell, C. Billinkoff, MSc, MBBS, S. Chun, MD, G. Dashi, MD, G. Ginocchio, HBSc, N. Hamilton, MD, M. Mardoquio, BHSc, A. Nikouline, MD, BMSc, L. Matukas, MD, MSc, S. Vaillancourt, BA, MD CM, MPH

St. Michael's Hospital, Toronto, ON

Background: Emergency departments (ED) provide routine care and testing for sexually transmitted infections (STIs). At St. Michael's Hospital ED, an inner-city downtown academic hospital, in Toronto, up to $25 \%$ of samples were being rejected in a month. This creates challenges which can lead to falsely reassuring patients, untreated infections, and further spreading infections in the community. To reduce the monthly sample rejection rate for diagnostic STI samples from the ED to less than 1\% by April 2020.

Aim statement: In collaboration with the microbiology department at our hospital we sought to identify the number and main reasons for rejection of STI samples that were collected in the ED using a Pareto chart. We focused on gonorrhea and chlamydia sample collection from all collected sites (i.e.: vaginal, urine, rectum, throat). We did not focus on serology based testing of STIs including syphilis and HIV. We tracked total STI samples collected per month as a balancing measure to monitor if the new workflow changes would discourage ED physicians from ordering the tests altogether.

Measures and design: We used Pareto charts, process mapping and fish bone analysis to better understand the reasons for STI sample rejection from Oct 2019 to Dec 2019. Most of the sample rejection took place because of incorrect collection kit being used, incorrect urine volume collection, inappropriate source for test, and no specimen received. To date we have implemented three interventions to reduce STI sample rejection: (1) we have improved stocking and labelling for NAAT/PCR swabs, and for gonorrhea culture swabs, (2) we simplified the computer order entry set for our ED to help physicians navigate the different STI order entry options, and (3) we held department wide educational rounds to improve physician comfort in ordering the correct swabs. Our average STI rejection rate between Mar and Dec 2019 was 11.0\% (107/974 collected), while collected data to between Jan and Oct 2020 was $8.5 \%$ (69/810 collected). We have not been successful in reaching our target STI test rejection rates.

Evaluation/results: Our next steps to further reduce rejection rates include (4) patient-facing posters for improved urine collection in patient bathrooms, and (5) making test labels more consistent between the specimen package and computer order entry.

Keywords: Emergency department laboratory testing, Quality improvement and patient safety, Sexually transmitted infections 


\section{LO17}

Guideline for management of healthy children with first episode of febrile neutropenia

C. Grandjean-Blanchet, MD, O. Ostrow, MD, S. Villeneuve, MD, C. Beck, MD, MSc, M. Cada, MD, MPH, D. Rosenfield, MD, M. Science, MD, M. Fantauzzi, BScN, S. Butchart, BScN

The Hospital for Sick Children, Toronto, ON

Background: While the management of febrile neutropenia in patients with cancer has clear, evidence-based guidelines, the management of previously healthy, immunocompetent children with a febrile illness and first episode of neutropenia is less understood and often similarly treated with empiric antibiotics and hospitalization. Many studies have shown that this population is at low risk of serious bacterial infections if they are well appearing with a short history of neutropenia. Therefore, less aggressive management should be considered in patients meeting low risk criteria. The aim of our quality improvement study was to decrease the number of unnecessary hospitalizations and empiric antibiotics prescribed by $50 \%$ for healthy, well appearing patients presenting to the emergency department (ED) with a first episode of febrile neutropenia (absolute neutrophil count $(\mathrm{ANC})<0.5 \times 10^{9} / \mathrm{L}$ ) over a 12 -month period

Aim statement: A team of stakeholders from Hematology, Infectious Disease, Pediatrics and Emergency Medicine was formed. A review of the literature, peer institutions and local practices on febrile neutropenia in healthy children was performed. A guideline for the management of healthy children with first episode of febrile neutropenia was developed and refined using PDSA cycles. Using the Model for Improvement, a family of measures was developed to assess project impact over time, as well as potential unintended consequences.

Measures and design: Baseline data was collected between June 2018 and January 2020. Nineteen low risk patients were identified. Data analysis revealed that $84 \%$ of these patients were hospitalized and/or received antibiotics. A knowledge gap surrounding the correct definition of severe neutropenia was also identified. Many patients were misdiagnosed with neutropenia by forgetting to count the bands in the ANC. This was addressed through education and pathway modifications. In January 2020, the guideline was launched for clinical use in the ED. Fourteen patients met low risk criteria. Hospitalization and/or antibiotics use for this population decreased to $21 \%$. All blood cultures were negative.

Evaluation/results: We contributed to improving the quality of care of this population by reducing potential harm from unnecessary hospitalizations and antibiotics in low risk patients. The development of this guideline led to improve resource stewardship and value-based care. Next steps include further iterations to the guideline to increase impact along with sustainability planning.

Keywords: Febrile neutropenia, Healthy children, Quality improvement and patient safety

\section{LO18}

Reducing overuse of urine cultures in the emergency department

S. Dowling, BA, MD, L. Saxinger, MD, A. Peterson, BSc, T. Nickonchuk, BSc, D. Pasay, BSc, R. Shkrobot, BSc

University of Calgary, Calgary, $\mathrm{AB}$

Background: Low value urine cultures (UCs) and treatment of asymptomatic bacteriuria drive unnecessary antibiotic use and increase health care costs. Opportunities exist to improve patient care and resource utilization by reducing unnecessary UCs. A prior study at our center showed that $44 \%$ of those treated for urinary tract infection (UTI) do not have UTI symptoms. The goal of the project was to reduce low value UCs in our tertiary care emergency department (ED) using a multimodal strategy-education, a clinical decision support tool and order set changes.

Aim statement: Executive endorsement was obtained for the project from ED leadership. Antimicrobial stewardship pharmacists met 1:1 with ED staff in June 2019. Using academic detailing techniques, barriers and facilitators to urine test ordering were assessed. The previously developed UTI pathway was introduced during these sessions and implemented in our ED. Barriers identified were addressed by aligning order sets with the UTI tool, educational rounds on UTI management. The main outcome was the number of UCs/100 ED visits in the 12 months pre and post intervention period (from June 1, 2018 to May 31, 2020). Data is also being captured on balancing measures include revisits and number of antibiotics prescribed. Ordering rates from other EDs in our zone served as the control.

Measures and design: The run charts demonstrate special cause variation starting in June 2019 correlating with the intervention period. Our analysis found a pre-intervention rate of $15.9 \mathrm{UCs} / 100 \mathrm{ED}$ visits compared to $12.3 / 100$ (absolute/relative reduction of $3.6 \%$ and $23 \%, \mathrm{p}=<0.001)$ in the post-intervention period. UC rates at the control sites were stable at $8.4 / 100$ during the same time period. Interrupted time series analysis confirmed the reductions were a function of the intervention site and study period. When considering ED volumes, the project would result in 401 fewer UCs/year (95\% CI 144-658).

Evaluation/results: This project resulted in a significant and sustained reduction in UCs in our ED. Keys to the success of this project include establishing a relationship with clinical nurse educators, pharmacists and the antimicrobial stewardship team to act as project champions and promote awareness of the problem. Academic detailing assisted with the education and roll-out of the UTI pathway as well as identifying barriers. In the future, site level audit data will be provided to ensure improvement is sustained and the project is currently being scaled up to other EDs in our zone.

Keywords: Low value care, Quality improvement and patient safety, Urine culture

\section{LO19}

Care gap analysis and development of care pathway to optimize management of early pregnancy complications

C. DeBiasio, BASc, K. Chen, MD, A. Pozgay, MD, S. Wilson, BSc, M. McKinnon, BSc HKin, G. Clapham, BA, S. Calder, MD, J. Dy, MD, E. Kwok, MD

University of Ottawa, Ottawa, ON

Introduction: Across many healthcare settings, Health Quality Ontario (HQO) Quality Standards outline specific goals and outcomes for care. In February 2019, HQO updated their Quality Standards on Early Pregnancy Complications (EPC) and Loss to better match evidence-based recommendations. At The Ottawa Hospital (TOH) Emergency Department (ED), we aim to identify possible care gaps between recent HQO Standards and care provided, to develop clinical-care pathway models (CPM) to address identified gaps.

Methods: Retrospective data collection and chart reviews were performed on $2018 \mathrm{ED}$ EPC encounters at TOH. 504 encounters were identified for EPC and were included for data analysis. The EPC CPM was developed by an interdisciplinary team, involving nursing educators, social workers, patient advocates, emergency physicians and obstetrician/gynecologists. The development process used focus group interviews with patient advocacy representatives and written summaries from patient complaints. 
Results: $455(89 \%)$ patients with EPC presented with vaginal bleeding. $22(4 \%)$ patients were offered initial pain management, and one $(<1 \%)$ patient received screening for sexually transmitted infections or intimate partner violence. $420(83 \%)$ patients received an appropriate medical interview, physical exam, and laboratory and imaging assessment. In the setting of an ectopic pregnancy, psychosocial support referrals were not offered. If a non-viable intrauterine pregnancy (IUP) was confirmed, appropriate medical or surgical management and referral to psychosocial supports were offered to all patients. Preliminary analysis highlights care gaps between HQO Quality Standards and TOH ED EPC care. The development process of CPM addressed these quality gaps. Nursing triage directives and CPM were devised. An education booklet was revised to reflect the inter-professional nature of the CPM.

Conclusion: Based on the quality care gaps identified, we developed a CPM that was suitable for EPC. This CPM provided the framework of integrated and coordinated interdisciplinary care. Our future work aims to disseminate the CPM, perform post-implementation analysis to confirm improvements in initial pain management, STI/IPV screening, and psychosocial support. Staff education on the clinical pathway will be critical to ensure outcome measures are meeting standards set by HQO.

Keywords: Care gap, Clinical pathway model, Early pregnancy complications

\section{LO20}

An assessment of the accuracy of the Simple Triage and Rapid Treatment method in the triage of patients in disaster and mass casualty incidents: a meta-analysis

S. Kirkland, MSc, U. Duarte Wisnesky, PhD, J. Meyer, BSc, S. Campbell, MLS, B. Rowe, MD, MSc, J. Franc, MD, MSc

University of Alberta, Edmonton, $\mathrm{AB}$

Introduction: For the triage of patients in disaster or mass casualty incidents (MCI), the Simple Triage and Rapid Treatment (START) algorithm is frequently used. Despite the ubiquitous use of START, there has been no systematic assessment of the diagnostic accuracy of START to identify patients to their appropriate treatment. The objective of this systematic review was to conduct an assessment of the accuracy and diagnostic characteristics of START in disaster or MCI settings.

Methods: A systematic search of the literature was conducted to identify studies assessing the accuracy of START in either true or simulated disaster or MCI settings in adult participants. Data on START diagnostic accuracy had to be included in the primary studies. Two independent reviewers assessed the eligibility of studies, quality assessment, and conducted data extraction. Conflicts were settled via discussion and disagreements were settled via third party mediator. Meta-analysis was performed using Stat59 (STAT59 Services Ltd, Edmonton, Canada), a fixed effects model and sub-classified using the START classification (e.g., red, yellow, green, black).

Results: After the removal of duplicates, 1820 studies were screened, of which, 32 studies were included in the review. While all of the studies included a reference standard, the majority of studies $(n=22)$ did not specify those details. Overall, the accuracy of START across all studies was $70.2 \%$ (95\% CI 69.8, 70.6). Over-triage rate was $9.6 \%$ $(95 \%$ CI $9.3,10.1)$, while overall under-triage rate was $6.3 \%(95 \% \mathrm{CI}$ $6.0,6.6 \%$ ). Triage accuracy for red cases was $82.5 \%$ (95\% CI 81.5 , 83.6), for yellow cases $69.7 \%$ (95\% CI $68.5,70.8)$, for green cases $86.4 \%$ (95\% CI 85.6, 87.1), and for black cases $82.1 \%$ (95\% CI 80.4, 83.8).

Conclusion: Although START is one of the most commonly used triage protocols for disasters, its accuracy overall is unknown. This study suggests that under varying situations, START accuracy may be too low to be considered reliable. Research should also be undertaken to inform disaster service providers regarding the comparative effectiveness of other triage algorithms.

Keywords: Accuracy, Disaster medicine, Triage

\section{LO21}

A survey of Canadian emergency physicians' experiences and perspectives during the COVID-19 pandemic

N. Gaucher, MD, PhD, E. D. Trottier, MD, A. Côté, MD CM, MSc, H. Ali, MD CM, S. Ali, MD CM

CHU Sainte-Justine, Université de Montréal, Montréal, QC

Introduction: The objective of this study was to explore Canadian Emergency Department (ED) physicians' experiences, concerns, and perspectives during the first wave of the COVID-19 pandemic.

Methods: This was a cross-sectional survey of ED physician members of Pediatric Emergency Research Canada and Canadian Association of Emergency Physicians. The survey included four themes: personal safety/responsibility to care; relationships with patients; ethical issues in pandemic care; institutional dynamics and communication practices.

Results: From June 25 to July 29, 2020, 187 participants completed the survey: $52 \%$ (70/157) were female, and 38\% (50/137) were 4049 years old. Participants reported a high moral obligation to care for patients (97/100, IQR 85-100) and concern regarding lack of personal protection equipment (PPE) (67/100, IQR 27-86). Fear of contracting COVID-19 changed how $82 \%$ (113/137) of respondents reported interacting with patients, while 97\% (138/142) reported PPE negatively impacted patient care. Despite reporting a high proportion of negative emotions $(84 \%, 326 / 389)$, respondents $(59 \%, 81 / 137)$ were not concerned/slightly concerned about their own mental health. ED physicians' top concerns included the potential for a second wave, Canada's financial situation, worldwide solidarity, and pandemic impact on youth mental health. Top facilitators to provide ED care included: teamwork, leadership, and clear communications strategies. Common barriers to providing effective ED care were confusing and changing guidelines, inadequate PPE, and inadequate ED set-ups/ environments.

Conclusion: Canadian ED physicians felt a very strong sense of responsibility to care, and dealt with various ethical dilemmas. Clear communication strategies, measures to ensure safety, and appropriate ED setups facilitate pandemic care. ED physicians were not very concerned about their own mental health; this requires further exploration.

Keywords: COVID-19 pandemic, Emergency physicians, Responsibility to care

\section{LO22}

Subjective pain and discomfort among stable blunt trauma patients transported with various prehospital spinal immobilization devices

D. Wong, MD, C. Vaillancourt, MD, MSc, M. Charette, MSc, E. Sabri, MSc, B. McLeod, MPH, S. Cheskes, MD, C. Macarthur, $\mathrm{PhD}, \mathrm{MBChD}$, M. Taljaard, PhD, I. Stiell, MD, MSc

University of Ottawa, Department of Emergency Medicine, Ottawa, $\mathrm{ON}$

Introduction: This study evaluated differences in pain and discomfort among stable patients with suspected c-spine injury following 
blunt trauma and transported with various prehospital spinal immobilization devices.

Methods: This was a cohort study nested within a stepped wedge cluster randomized trial to evaluate the use of the Canadian C-Spine Rule by paramedics. The trial took place in 11 Ontario communities and evaluated all consecutive eligible stable adult patients at risk for cspine injury. We included a subset of patients where an immobilization device was used during transport. Paramedics' standardized documentation of pain and discomfort scores (10-point ordinal scale) were completed when transferring care to emergency department staff. Our primary analysis compared pain and discomfort (dichotomized at prespecified cut-points of 4) associated with the use of each immobilization device using random effects logistic regression adjusted for age, sex, language barrier, and immobilization time, accounting for intra-cluster and intra-period correlation reported as Odds Ratios (ORs) and 95\% confidence intervals (95\% CI). Secondary analyses used random effect linear regression of continuous scores.

Results: In total, 3126 patients were evaluated: median age 60 years (range 16-103 years), female 50\%, MVC 43\%, fall 43\%, immobilized with c-collar alone $(\mathrm{n}=1390)$, plus backboard $(\mathrm{n}=1555)$, or scoop device $(n=181)$. Mean immobilization time in minutes with standard deviation (SD) were: for c-collar alone (50 min; SD 40), plus backboard (40 min; SD 27), or scoop device (41 min; SD 27). Mean overall pain (5.1/10; SD 2.9) and discomfort (5.7/10; SD 2.8). Adjusted ORs for pain and discomfort (dichotomous $>4$ and $<=4$ ) were: (1) c-collar alone vs. with scoop device (OR 0.5 ; $95 \%$ CI 0.4 $0.8, \mathrm{p}=0.002 ;$ OR $0.9 ; 0.6-1.3, \mathrm{p}=0.53)$; (2) c-collar alone vs. with backboard (OR 0.6; 0.4-0.8, p < 0.001; OR 0.7; 0.5-0.9, p =0.01); and (3) backboard vs. scoop (OR 0.9; 0.7-1.3, p = 0.74; OR 1.3; 0.9$1.8, \mathrm{p}=0.15)$. Results from our secondary analyses treating pain and discomfort as continuous scores were similar.

Conclusion: Pain and discomfort were least when using a c-collar alone. There was no statistical difference in pain or discomfort comparing backboard to scoop devices. This study provides further evidence, from a large multi-center cohort, that spinal immobilization is not benign and causes significant pain and discomfort in the traumatized patient.

Keywords: Prehospital, Spinal immobilization device, Trauma

\section{$\mathbf{L O 2 3}$}

Alternative care models for paramedic patients from long-term care centers; a national survey of Canadian paramedic services

S. Leduc, MSc, G. Wells, PhD, V. Thiruganasambandamoorthy, MSc, MBBS, P. Kelly, C. Vaillancourt, MD, MSc

Ottawa Paramedic Service, Ottawa, ON

Introduction: Long-term care (LTC) patients do poorly when transported to the emergency department (ED) for care. Community paramedic programs can deliver enhanced care in their place of residence, yet few such programs are reported in the literature. We conducted a national cross-sectional survey of land ambulance services to understand if such programs exist in Canada, and what the perceived needs and priorities are for future programs.

Methods: We emailed a 46-question electronic survey to paramedic services across Canada. We used a modified Dillman method to deliver our survey to the individuals identified by service leadership as the most appropriate people to complete the survey and up to three reminders were sent. We asked services about their characteristics, current ED diversion programs, existing diversion programs specific to LTC patients, priorities for future programs, the potential impact of such programs, and what the feasibility and barriers are to implementing programs that treat LTC patients on-site, avoiding an ED visit.
Results: We received responses from 50 services across Canada, providing services to $73.5 \%$ of the total population. Almost half were municipally run $(48.0 \%), 68.0 \%$ covered a mix of low and high population density, and $28.0 \%$ employed self-regulated paramedics. Almost a third $(30.0 \%)$ had existing treat-and-refer programs, and $65.5 \%$ reported transporting to destinations other than an ED. Almost all respondents (98.0\%) felt the need for programs to treat LTC patients on site, avoiding an ED visit, and $36.0 \%$ had such existing programs. The top priorities for future programs were support for patients being discharged (30.6\%), extended care paramedics $(24.5 \%)$, and respiratory illness treat-in-place program $(20.4 \%)$. The highest potential impact was expected from support for patients being discharged $(62.0 \%)$ and respiratory illness treat-inplace programs $(54.0 \%)$. Required changes in legislation $(36.0 \%)$ and required changes to the current system of medical oversight $(34.0 \%)$ were identified as the top barriers to implementing such programs.

Conclusion: The number of current community paramedic programs treating LTC patients on site does not match the identified need for such programs, and little scientific evidence exists that evaluates existing programs. There are several program models believed to have high impact, but significant barriers to broad adoption exist including current legislation and structure of medical oversight.

Keywords: Community paramedicine, Crowding, Long-term care

\section{LO24}

Ambulance usage among people experiencing homelessness in Ontario

S. Strobel, MD, W. Deng, BSc, I. Burcul, BSc, R. Hossain, MD

Cornell University, Ithaca, New York

Introduction: Previous studies have found that people experiencing homelessness access emergency departments (ED) up to 4 times more than individuals who are housed, and that homelessness is associated with low acuity ambulance use. We leverage administrative data to analyze associations between ambulance use and housing status.

Methods: Administrative health data of all contacts by people experiencing homelessness presenting to EDs within Ontario, Canada, from 2010 to 2017 was analyzed. Individuals experiencing homelessness were identified by the designation of an "XX" postal code at the time of ED registration. Our outcomes include the number of people experiencing homelessness in Ontario who present to ED by ambulance, which we stratify by year and by week from 2010 to 2017 and triage scores, in the context of housing status and ambulance use. We compare these to a sample of persons who have not experienced homelessness.

Results: We observe 603,995 presentations by people experiencing homelessness and 1.5 million presentations by homed patients to EDs in Ontario from 2010 to 2017. Ambulance presentations occurred in $40 \%$ (std $=3.9 \%$ ) of ED visits by people who experienced homelessness compared to only $13.3 \%$ ( $\mathrm{std}=0.8 \%$ ) of visits by homed persons. The average triage score for ambulance-using people who experienced homelessness on presentation to ED was 2.71, compared to 2.63 for the homed ambulance-using population with CTAS scores distributed to less acute presentations relative to the homed population $(\mathrm{t}<0.05)$. Complaints of persons who experience homelessness are also significantly different relative to the homed with top complaints relating to psychiatric issues. People who experienced homelessness had a $16 \%(\mathrm{t}<0.05)$ higher probability of presenting at their next visit by ambulance relative to the homed; however, those persons who used an ambulance were $6 \%(\mathrm{t}<0.05)$ less likely to represent by ambulance at their subsequent visit compared to homed individuals. 
Conclusion: Ambulance use among persons experiencing homelessness is much higher relative to the homed population. The distribution of triage scores of those experiencing homelessness skews towards less acute presentations relative to the homed. There are significant differences between these groups in presentation complaints and return visits associated with ambulance use. Strategies that target the health of persons experiencing homelessness may also disproportionately reduce ambulance overuse.

Keywords: Ambulance, Homelessness

\section{LO25}

Machine learning versus usual care for diagnostic and prognostic prediction in the emergency department: a systematic review

H. Kareemi, MD, C. Vaillancourt, MD, MSc, H. Rosenberg, MD, K. Fournier, MSI, K. Yadav, MD, MSc

University of Ottawa, Department of Emergency Medicine, Ottawa, ON

Introduction: Machine learning (ML) models have shown promising diagnostic and clinical prediction performance in other medical fields yet remain largely unexplored in emergency medicine. We sought to determine whether ML models perform better than usual care in diagnostic and prognostic prediction for emergency department (ED) patients presenting with various clinical conditions.

Methods: In this systematic review, two independent abstractors searched MEDLINE, Embase, Central, and CINAHL from inception to October 17, 2019. A third reviewer adjudicated disagreements. We reviewed reference lists and grey literature including conference proceedings and trial registries. We included studies comparing diagnostic and prognostic prediction of ED patients by ML models to usual care methods (triage-based scores, clinical prediction tools, clinician judgment) using predictor variables readily available to ED clinicians (e.g. vital signs, demographics, basic investigations). Using a pilot-standard form, we extracted commonly reported performance metrics of model discrimination and classification. We used the PROBAST tool for risk of bias assessment and followed PRISMA guidelines.

Results: Among 1,656 unique records, we included 23 studies involving 16,274,647 patients across 36 observational cohorts. The most common ML models were neural network $(n=13)$, random forest $(n=11)$, gradient boosting $(n=8)$, and support vector machine $(\mathrm{n}=5)$. The most commonly studied conditions were "any chief complaint" $(n=7)$, chest pain $(n=5)$, sepsis $(n=3)$, or gastrointestinal bleed $(n=2)$. In all 7 diagnostic studies, ML models outperformed usual care in all performance metrics. In 6 studies predicting in-hospital mortality, the best-performing ML models had better discrimination (AUROC 0.74-0.94) than any clinical decision tool (AUROC 0.68-0.81). In 4 studies predicting hospitalization, ML models had better discrimination (AUROC $0.80-0.83$ ) than triagebased scores (AUROC 0.68-0.82). Clinical heterogeneity precluded meta-analysis. Most studies had high risk of bias due to lack of external validation, low event rates, and insufficient reporting of calibration.

Conclusion: This systematic review suggests that ML models may have better prediction performance than usual care for ED patients with a variety of clinical presentations and outcomes. Prospective interventional trials assessing patient-centered outcomes using ML models may have a significant impact on the future of emergency medicine practice.

Keywords: Artificial intelligence, Machine learning, Prediction

\section{LO26}

Communicating wait-time information in Canada: a scoping review of public-facing wait time displays in the emergency department

R. Rathagirishnan, BHSc, I. Birchall, BSc, A. Latimer-Cheung, BSc, $\mathrm{MSc}, \mathrm{PhD}, \mathrm{S}$. Tse, MD, K. Cheung, MD, MSc

Queen's University, Kingston, ON

Introduction: Waiting is a common experience for patients during an Emergency Department (ED) visit. While high acuity patients are seen with little delay, low acuity patients may experience dissatisfaction from prolonged wait times. One strategy to improve patient experience involves reducing the actual wait time. An alternate, less resource intensive strategy, involves changing the perception of the wait. Providing realistic expectations of wait times using publicfacing wait time displays (WTDs) may improve patient experiences. The scope of the use, quality, and effectiveness of WTDs, however, are unknown. The primary objective of this study is to identify and characterize existing WTD tools in Canada. This will inform future studies to determine patients' preferred message characteristics and user experience features in WTDs.

Methods: A systematic scoping review was performed using Levac et al.'s (2010) five-stage methodology. The guiding research question was "how are existing real-time WTDs communicating information to Canadian patients in the Emergency Department?" A systematic online search of all Canadian EDs was completed to identify EDs with real-time WTDs, assess their message characteristics, translations offered, availability of multi-site information, and accessibility features. Data were summarized through descriptive statistics.

Results: Sixty [9.3\% (95\% CI 7.1-11.5\%)] of the 645 Canadian EDs identified provide public-facing real time WTDs. Thirteen of these [21.7\% (95\% CI $11.2-32.1 \%)]$ were associated with a single proprietary system. Distribution of WTDs differ across Canada with Ontario [35\% (95\% CI 24.2-47.7\%)], Alberta [33.3\% (95\% CI 22.7-45.9\%)], and British Columbia [10\% (95\% CI 9.3-28\%)] employing WTDs most frequently. Eighty-eight percent (95\% CI 80.2-96.4\%) provide an "average" wait time, defined as the mean time between being triaged and seeing a doctor. Other common characteristics included graphical trend data [32\% (95\% CI $21.3-44.2 \%)$ ], number of patients waiting [27\% (95\% CI 17.1-39\%)], longest wait time [12\% (95\% CI $5.7-22.2 \%)]$, and expected length of stay [10\% (95\% CI 4.7-20.1\%)]. Sixty-three percent (95\% CI 50.6-74.4\%) of WTDs provide a combination of these methods to inform wait times.

Conclusion: Currently there is limited but emerging use of WTDs in Canada. Given that there is considerable variability in the information communicated through these tools, effectiveness of these displays and their content need to be determined.

Keywords: Communication tools, Patient experience, Wait times

\section{LO27}

Where did all the patients go? The impact of reduced patient volumes on Calgary emergency department flow parameters

R. Killam, BSc, E. Lang, MD

University of Calgary, Calgary, AB

Introduction: Emergency departments (EDs) in Calgary, Alberta prepared for a surge in patient visits during the first wave of the COVID-19 (SARS-CoV-2 virus) pandemic. A state of local emergency was declared in Calgary on March 15, 2020. This marked the starting point of public health measures aimed at "flattening the curve" in hopes that infection rates wouldn't overwhelm the 
healthcare system. The objective of this study was to demonstrate changes in operational metrics in all four adult Calgary EDs within the first 9 weeks of the pandemic. The objective of this study is to investigate over-time trends in ED visit volumes and departmental operations between March 15 and May 24 of 2020 compared to the same time period of 2019 .

Methods: The authors retrospectively reviewed the Calgary Zone Alberta Health Services ED database for visits between March 15 and May 24 for 2019 and 2020. The data from 2020 showed Calgary ED admissions for the four adult EDs during the first 9 weeks of the COVID-19 pandemic. Comparative analysis was used to evaluate changes in averages in various patient demographics as well as key outcomes in ED flow parameters, such as time from triage to seeing ED physician, time to decide to admit, ED length of stay for admissions, and ED length of stay for discharges. The changes observed were dramatic, further statistical analysis was deemed unnecessary.

Results: The study examined 61,388 visits to adult EDs in the Calgary zone between March 15 to May 24 of 2019 compared to 43,934 patient visits for the same time period in 2020, a $28 \%$ decrease. This reduction correlated with a $50 \%$ decrease in the time from triage to seeing an ED doctor (121.1 min, 2019 to $60.2 \mathrm{~min}, 2020$ ). There was also a $40 \%$ decrease in ED length of stay for admitted patients (14.1 h, 2019 to $8.4 \mathrm{~h}, 2020)$. The time to decide to admit decreased by $57 \%(5.3 \mathrm{~h}, 2019$ to $2.3 \mathrm{~h}, 2020)$. This coincided with a $68 \%$ hospital occupancy compared to the normal $105 \%$.

Conclusion: There was an evident decrease in patient visits to Calgary EDs during the initial phase of the COVID-19 pandemic. Interestingly, the reduction in patient volumes correlated with a nonlinear decrease in operational flow parameters, with several CAEP targets being met at the four adult EDs in Calgary. Reductions in the length of stay prior to admission was due to the combination of decreased patient volumes along with interdepartmental efforts from admitting services as patients moved to units faster.

Keywords: COVID-19, Emergency department flow, Quality improvement and patient safety

\section{LO28}

The impact of employing primary healthcare professionals in emergency department triage on patient flow outcomes: a systematic review

R. Alder, BSc, A. Abou-Setta, MD, PhD, L. Copstein, MD, MPH, N. Al-Yousif, MD, R. Suss, MD, R. Zarychanski, MD, MSc, M. Doupe, PhD, S. Berthelot, MD, J. Mireault, MD, P. Tardif, MD, N. Askin, MLIS, T. Buchel, MD, R. Rabbani, PhD, C. Shimmin, MSc, J. Edwards, O.T. Reg. (MB), MHA, BOT, CHE, G. Halas, PhD, W. Sevcik, MD, A. Tricco, PhD, B. Rowe, MD, A. Chochinov, MD, M. Jeyaraman, $\mathrm{MD}, \mathrm{PhD}$

University of Manitoba, Winnipeg, MB

Introduction: Healthcare systems worldwide encounter overcrowding in emergency departments (ED) that adversely affects health outcomes and leads to patient and provider dissatisfaction. Our objective is to identify, critically-appraise and summarize evidence on the impact of employing primary healthcare professionals (PHCPs: family physicians/general practitioners (GP), nurse practitioners (NP) and nurses with expanded role) in the ED triage, on patient flow outcomes.

Methods: We searched Medline (Ovid), EMBASE (Ovid), Cochrane Library (Wiley) and CINAHL (Ebsco) (inception to January 2020). Our primary outcome was the time to provider initial assessment (PIA). Secondary outcomes included time to triage, proportion of patients leaving without being seen (LWBS), length of stay (ED LOS), proportion of patients leaving against medical advice (LAMA), number of repeat ED visits, and patient satisfaction. Two reviewers selected studies, extracted data, and assessed study quality using the NICE quality assessment tool. Descriptive data synthesis was performed as meta-analysis was not possible due to heterogeneity. PROSPERO trial registration number: CRD42020148053.

Results: From 23,947 records, we included 40 comparative studies including 10 randomized controlled trials (RCTs) and 13 pre-post studies. PHCP interventions were led by triage nurses with expanded roles $(n=23)$, nurse practitioners $(n=14)$, and triage family physicians/general practitioners $(n=3)$. In all studies PHCP-led intervention effectiveness was compared to the traditional nurse-led triage model. Median duration of the interventions was 6 months. Study quality was generally low (confounding bias); 7 RCTs were classified as moderate quality. Evidence from RCT's $(n=8)$ as well as other study designs showed a significant decrease in ED LOS favoring the PHCP-led interventions. Most studies reported that PHCP-led triage interventions decreased the PIA (13/14), ED LOS (29/30), proportion of patients LWBS (8/10), time to triage (3/3), and repeat ED visits $(5 / 6)$, and increased the patient satisfaction $(8 / 10)$. The proportion of patients LAMA did not differ between groups (3/3). Conclusion: Overall PHCP-led triage interventions improved key ED patient flow metrics. There was a significant decrease in ED LOS irrespective of the study design, favoring the PHCP-led interventions. Evidence from well-designed high quality RCTs is required prior to widespread implementation.

Keywords: ED patient flow, ED triage, Primary healthcare provider

\section{LO29}

Does emergency department opioid prescribing correlate with community opioid-related deaths (the opioid epidemic)

J. Hayward, MD, MSc, G. Innes, MD, MSc

University of Alberta, Edmonton, AB

Introduction: Since 2016 there has been a dramatic increase in opioid related deaths, which has been attributed in part to physician opioid prescribing behaviour. This is a preliminary analysis of administrative data examining ED opioid prescribing over an 8-year period. We studied a random sample of ED patients who visited Alberta's 15 tertiary, urban and regional adult hospitals from 2011 to 2019.

Methods: Index ED visits for patients over 18 years of age were studied. We excluded patients with cancer or palliative care, patients with pre-existing opioid use disorders (OUD), pregnant patients and repeat ED visits. We acquired data from the Alberta Health Services (AHS) enterprise data warehouse and obtained ED opioid prescriptions in the pharmaceutical information network (PIN) database. We also captured patient demographic and triage data, material and social deprivation scores, CIHI health profile groupings and comorbidity scores based on ICD-10 data to assure that changes in ED populations were not driving changes in opioid utilization. We summarized rates and proportions of patients receiving post-ED opioids, identified specific opioids prescribed, and documented trends over time. We calculated standardized mean differences to determine the significance of observed changes in prescribing patterns.

Results: We studied 25,867 eligible patients, mean age 43.2 years, $53.4 \%$ male. During the study, there were no significant changes in age, sex, EMS arrival rates (13\%), presenting complaint category, or geographic visit distribution (41.3\% urban, $22.6 \%$ regional, $18.7 \%$ tertiary). Patient comorbidity scores, health profile groups, material and social deprivation scores, and rates of mental health and 
substance use diagnoses were similarly consistent during the study period. Overall, $8.2 \%$ of eligible ED patients received an opioid prescription, including 1484 codeine preparations (5.7\%), 353 Tramadol (1.4\%), and $1.1 \%$ potent opioids (morphine, fentanyl, hydromorphone or oxycodone). We observed no significant change in the overall rate of opioid prescription, the total morphine equivalents dispensed, or the distribution of opioid types prescribed during the study period $(\mathrm{SMD}=0.094)$.

Conclusion: Alberta ED opioid prescribing has remained consistent over time. Despite large increases in community opioid deaths, we did not identify potentially corresponding changes in ED opioid prescription.

Keywords: Fentanyl, Opioids, Overdose

\section{LO30}

\section{Attitudes, behaviour, and comfort of Canadian emergency physicians in caring for 2SLGBTQI+ patients}

K. Lien, BSc, M, V. Ng, MD, MHPE, MSc, B. Vujcic, BSc

Western University, London, ON

Introduction: Physicians working in emergency departments (ED) will interact with two-spirited, lesbian, gay, bisexual, transgender, queer/questioning and intersex (2SLGBTQI+) persons as colleagues and patients. These patients have unique healthcare needs and encounter negative experiences when seeking medical care, leading to potentially poorer health outcomes and inequities. This study aims to explore the attitudes, behaviour, and comfort of Canadian emergency medicine (EM) physicians in caring for 2SLGBTQI+ patients.

Methods: An anonymous survey was distributed to EM staff physicians and residents through the Canadian Association of Emergency Physicians distribution network and social media channels using a modified Dillman approach. Demographics information was collected, and participants were asked about their comfort, current knowledge, and desire to gain new knowledge in caring for 2SLGBTQI+ patients. Personal perceptions and practice patterns in treating cisgender-heterosexual (cis-het) and 2SLGBTQI+ patients were analyzed using five-point Likert scales. Residents were asked additional questions regarding availability of learning experiences during their training program. Descriptive statistics, two samples t test and Chi-square tests were performed (SPSS 15).

Results: A total of 266 out of 324 submitted surveys were completed in entirety and included in the final analysis consisting of $229(86 \%)$ staff physicians and $37(14 \%)$ residents. 97\% $(\mathrm{n}=258)$ of all respondents believed that 2 SLGBTQI+ patients deserve the same level of quality care from medical institutions as other patients. Further, $83 \%(\mathrm{n}=221)$ respondents agreed that they would like to increase their knowledge in taking care of 2SLGBTQI+ patients, while $34 \%(n=91)$ agreed that performing physical examinations on transgender or intersex patients was more challenging than on cis-het patients. Among resident respondents, 54\% indicated a lack of didactic teaching devoted to 2 SLGBTQI+ care during residency $(n=20 / 37)$, while $38 \%$ encountered discrimination towards 2SLGBTQI+ patients, with most negative comments coming from senior faculty and nursing staff.

Conclusion: This study suggests that Canadian EM physicians feel that 2SLGBTQI+ patients deserve equitable care when compared to cis-het patients. Future work should focus on educational needs and curricular enhancements in both residency programs and continuing professional development for physicians to improve care for 2SLGBTQI+ patients in the ED.

Keywords: 2SLGBTQI+, Emergency medicine, Health equity

\section{LO31}

The impact of the COVID-19 pandemic on mental health and substance use visits to Canadian emergency departments

D. Barbic, MD, MSc, F. Scheuermeyer, MD, MSc, A. Chakraborty, PhD, MBBS, E. Grafstein, MD, S. Nolan, MD, S. Barbic, PhD, W. Honer, MD

UBC, Vancouver, BC

Introduction: The COVID-19 pandemic has altered the rates of presentation and outcomes of individuals presenting to emergency departments (EDs). The impact of the pandemic on persons with mental health and substance use (MHSU) disorders presenting to the ED is unknown.

Methods: The Vancouver Coastal Health Authority (VCH) serves a population of approximately 1.2 million people with six hospitalbased EDs. The region is the most densely populated region in Canada. For this study, visits were assessed by triage codes, and our team was specifically interested in mental health and substance use complaints. We collected the following aggregate data for each of the mental health and substance use codes: demographic (median age, number of male/female patients), Triage presentation, Outcome (median length of stay, number of patients admitted to hospital). The primary outcome of this study was to describe the impact of the COVID-19 pandemic on the number of ED presentations for people with MHSU disorders. A secondary objective was to explore the impact of the COVID-19 pandemic on two pre-defined, high risk subgroups (suicidal ideation; drug overdose).

Results: For the study time periods (Mar 1 to Oct 1 , for the years 2015-2020) there were 1,241,963 total visits, and 206,994 average annual visits to the six EDs. For 2020, compared to the previous 5 years, the overall visits for MHSU triage codes decreased across the region $(-13 \%)$. However, we observed an $11 \%$ relative increase in the number of persons presenting for mental health and substance use concerns during this same period. The proportions of MHSU ED presentations for suicidal ideation and drug overdose, compared to the previous 5 years, were unchanged.

Conclusion: In this historical comparison of six EDs serving a population of 1.2 million individuals, we observed a notable increase in MHSU presentations during the initial COVID period in this region. In order to optimize the health outcomes and experiences of this population, further research is required to explore the clinical and service delivery implications of these findings.

Keywords: COVID-19, Mental health, Substance use

\section{LO32}

Does hydronephrosis severity identify low-risk ureteral colic patients appropriate for trial of spontaneous passage without CT imaging

G. Innes, MD, MSc, A. McRae, MD, PhD, D. Lane, MD, PhD, K. Lonergan, BSc, F. Scheuermeyer, MD, MSc

University of Calgary, Calgary, AB

Introduction: In emergency department (ED) patients with presumed ureteral colic, the associations of hydronephrosis with stone size and outcomes are unclear. Our goal was to determine the ability of hydronephrosis to differentiate low-risk patients appropriate for trial of spontaneous passage from high-risk patients who require urgent CT imaging.

Methods: We evaluated consecutive ED patients with CT-proven ureteral stones at nine hospitals. We assessed classification accuracy and prepared regression models to evaluate the relationship of 
hydronephrosis with large stones $(>5 \mathrm{~mm}$ ). In the subgroup of patients attempting spontaneous passage, we used hierarchical Bayesian regression to estimate the linear association of hydronephrosis severity with passage failure, defined by the need for rescue intervention within 60 days. We also estimated its effect on posterior probability versus prior probability of passage failure.

Results: In 3251 patients, $70 \%$ male and mean age 51, the adjusted probability of a large stone was $13 \%, 25 \%, 53 \%$ and $78 \%$ for patients with absent, mild, moderate and severe hydronephrosis. Among 2148 patients attempting spontaneous passage, prior probability of passage failure was $22.4 \%$ and posterior probabilities were $15.5 \%, 21.5 \%$, $26.6 \%$ and $41.1 \%$ in the four hydronephrosis categories. [Absent or mild] hydronephrosis identified a large subset of patients $(64 \%)$ with a low passage failure rate $(\sim 13 \%)$ who may be appropriate for discharge without CT imaging. Patients with moderate hydronephrosis had minimally increased passage failure (see above), and those with severe hydronephrosis had substantially increased posterior probability of passage failure.

Conclusion: Absent and mild hydronephrosis identify low-risk patients unlikely to benefit from CT imaging or early intervention. Moderate hydronephrosis is a weak positive finding that does not substantially change the probability of passage failure. Severe hydronephrosis is a high risk finding that should trigger CT imaging and referral. Differentiating [absent-mild] from [moderate-severe] hydronephrosis provides modest risk stratification value, but more specific grading is not prognostically helpful.

Keywords: Hydronephrosis, Outcomes, Renal colic

\section{LO33}

Is pain really the fifth vital sign? Removal of pain from CTAS improves CTAS prediction of patient outcomes

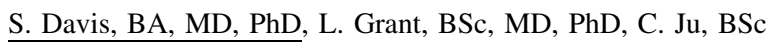

McGill University, Montreal, QC

Introduction: The vast majority of triage scales use pain as a criteria when defining patient acuity. The Canadian Triage Acuity Scale (CTAS) is no exception, as pain is used as a first order modifier. This means that self-reported pain is used to uptriage a significant number of patients in the ED. Unfortunately, pain is an inherently subjective and difficult to measure variable. The current study aimed to assess if the inclusion of pain in CTAS made acuity assessment more or less accurate.

Methods: This was a retrospective cohort study of all patient visits to a large tertiary hospital over 20 years. All patients CTAS scores were recorded on arrival, as well as all vitals and primary complaints. We built an algorithm that recreated CTAS, and then removed the pain scale from the CTAS score. We then compared the CTAS score using pain to the CTAS score with pain removed on our outcome variables. The three main outcome variables were admission to hospital, admission to ICU, and death in the ED. Subgroup analyses were also done for central vs peripheral pain, as well as presenting complaints of chest pain, abdominal pain, and headache.

Results: There were a total of 639,310 visits during the study period. The mean CTAS score including pain was 3.7 compared to a mean score of 4.43 without including pain. Higher pain scores were correlated with a lower age $(-0.135, \mathrm{p}<0.001)$, male gender $(0.052$, $\mathrm{p}<0.001)$, and arrival by walk-in $(0.091, \mathrm{p}<0.001)$. Using ROC analysis, it was found that CTAS without pain was a better predictor of admission (AUC $=0.763$, CI $0.761-0.765$ ) than CTAS with pain $(\mathrm{AUC}=0.747$, CI $0.745-0.749)$. The same results were found for death, with CTAS without pain being a better predictor of death $(\mathrm{AUC}=0.759, \mathrm{CI} 0.758-0.761)$ than CTAS with pain $(\mathrm{AUC}=0.745$, CI 0.743-0.749). The AUC for predicting ICU admission was not significantly different. Further subgroup analyses showed similar results for patient with acute pain vs chronic pain, as well as subsets of patients with chest pain. Non-significant differences were found for patients with abdominal pain.

Conclusion: Overall, these results support the removal of pain as part of the assessment of patient acuity during triage. Pain does not appear to be a useful predictor of patient outcomes. We would however caution removing any pain assessment from triage, as pain control at triage is still an important goal, even if not predictive of outcomes. Keywords: CTAS, Pain assessment, Triage

\section{LO34}

Novel emergency medicine virtual consultation and referral service for long-term care residents

C. Wallner, BSc, MD, MSc, M. Welsford, MD, M. Panju, MD, MSc, k. Winter, BSc, MBA, A. Papaioannou, MD, H. Boyd, MD,

S. Mondoux, MD, K. DeWit, MD, MSc, M. Mercuri, PhD, K. Drake, MN, J. Burgess, BN, MSc, S. Wong, MD, K. OHalloran, BN, MN

\section{McMaster University, Hamilton, ON}

Introduction: In response to COVID-19, a city-wide interdisciplinary team involving community organizations and hospital partners proposed an innovative solution to provide enhanced acute care in longterm care (LTC). The LTC-CARES program (Consults and Recommendations for Emergency and Support services) was launched in April 2020 and connects LTC residents, their families, physicians, nurses and nurse practitioners to Emergency Department (ED) physicians using virtual care technology. Initial ED virtual consultation could enable lab and imaging access, as well as expedited follow-up (within 24-48 h) via virtual consults with numerous specialties. The aim of this study was to reduce unnecessary transfers to hospital.

Methods: A prospective database was created to log all LTC-CARES Emergency Physician consults in the first 6 months of the program. Consults were logged and patients were followed to document their testing and treatment. The primary outcome was the proportion of LTC residents referred to the program who were immediately transported to the ED after the LTC consultation (arrive in the ED within $4 \mathrm{~h}$ of request for consultation).

Results: In the initial 6 months, 89 consults from 22 LTC facilities were managed through the program. Most consults were related to medical complaints $(70 \%, \mathrm{n}=62)$, followed by injury $(17 \%, \mathrm{n}=15)$, and complications of $\mathrm{GI} / \mathrm{GU}$ tubes or intravenous access $(13 \%$, $\mathrm{n}=12)$. The majority, $66 \%(\mathrm{n}=59)$, did not require ED transfer. Of those who remained in LTC, half $(n=23)$ were referred for further specialty follow up primarily General Internal Medicine, Gastroenterology, and Orthopedics. In addition, seven patients were referred directly to Diagnostic Imaging for complications of gastric tubes $(\mathrm{n}=4)$, nephrostomy tube $(\mathrm{n}=1)$, or PICC lines $(\mathrm{n}=2)$.

Conclusion: Providing optimal care for frail and elderly individuals in the ED is challenging during COVID-19. We have initiated a novel patient-centered service for LTC residents which has avoided ED presentation in the majority of cases.

Keywords: Long-term care, Telemedicine. 8

\section{LO35}

Barriers and facilitators to nursing delirium screening in older emergency patients: a qualitative study using the Theoretical Domains Framework 
D. Eagles, MD, MSc, W. Cheung, MD, MMed, T. Avlijas, MSc, RN, K. Yadav, MD, MSc, R. Ohle, MSc, MBBCh, MA, M. Taljaard, PhD, F. Molnar, MD, MSc, I. Stiell, MD, MSc

Department of Emergency Medicine, University of Ottawa, Ottawa, $\mathrm{ON}$

Introduction: Delirium is present in $10 \%$ of older emergency department (ED) patients. It is vastly under-recognized, in part due to lack of standardized screening. Understanding local context and barriers to delirium screening are integral for successful implementation of a delirium screening protocol. We sought to identify barriers and facilitators to delirium screening in older ED patients among nurses.

Methods: We conducted semi-structured, face-to-face interviews based on the Theoretical Domains Framework (TDF), established theoretical constructs designed to identify determinants of behavior change that could impact uptake of evidenced based practice. We used a purposive sampling strategy interviewing bedside nurses, nurse educators and managers at two academic EDs in 2017 until data saturation was reached (defined as no new themes for three successive interviews). Our target behaviour was delirium screening of older ED patients. Interviews were audio recorded, transcribed verbatim and uploaded into NVIVO, a qualitative analysis program. Direct content analysis was utilized. Two research assistants independently coded transcripts iteratively, disagreements were resolved by a third researcher. Fleiss' Kappa (k) was calculated for interrater reliability. Relevant domains were identified based on frequency of belief statements, existence of conflicting beliefs and evidence of strong beliefs.

Results: We achieved data saturation after 15 interviews. Participants were $73.3 \%$ female, between 25 and 64 years of age and had median 20 (range 4-30) years of experience. A total of 717 utterances were coded into 14 domains. Interrater reliability was $\kappa=0.78$. Three dominant themes emerged: (1) delirium was recognized as an important clinical problem, but was not clinically prioritized because of competing demands, lack of time, and heavy workload; (2) there was a falsely elevated self-perception of knowledge and ability to recognize delirium; and (3) hospital culture was a strong influencer of behaviour.

Conclusion: This qualitative study based on the TDF explored barriers and facilitators to delirium screening in older ED patients among nurses. We found that delirium screening was not clinically prioritized; there were knowledge gaps which were incongruent with perceived capabilities; and hospital culture impacted screening behaviour. Successful adoption of a delirium screening protocol must address these barriers and facilitators to achieve meaningful changes. Keywords: Delirium, Emergency department, Screening

\section{LO36}

Efficacy and safety of tranexamic acid in acute traumatic brain injury: a systematic review and meta-analysis of randomized controlled trials

K. Al Lawati, BHSc, MD, S. Sharif, BHSc, MD, S. Al Maqbali, BHSc, MD, H. Al Rimawi, BHSc, MD, A. Petrosoniak, BHSc, MD, MSc, B. Rochwerg, BHSc, MD, MSc

McMaster University, Hamilton, ON

Introduction: Tranexamic Acid (TXA) is used for a number of indications, including blunt trauma, based on evidence that it decreases bleeding and improves survival. Its role in acute traumatic brain injury (TBI) is less certain. With the publication of a large randomized controlled trial (RCT) suggesting that tranexamic acid (TXA) may improve head-injury related deaths, we aimed to determine the safety and efficacy of TXA in acute traumatic brain injury (TBI).

Methods: In this systematic review and meta-analysis, we searched MEDLINE, PubMed, EMBASE, CINHAL, ACPJC, Google Scholar, and unpublished sources from inception until June 24th, 2020 for randomized controlled trials comparing TXA and placebo in adults and adolescents ( $\geq 15$ years of age) with acute TBI. We examined the reference list of full-text articles for additional relevant studies. We screened studies and extracted summary estimates independently and in duplicate. We assessed RoB, independently and in duplicate using a modified Cochrane RoB tool for which each domain is rated as "low", "probably low", "high", or "probably high". We assessed the quality of evidence using the grading of recommendations assessment, development, and evaluation approach. This study is registered with PROSPERO (CRD42020164232).

Results: Nine RCTs enrolled 14,747 patients. Compared to placebo, TXA had no effect on mortality (RR $0.95 ; 95 \%$ CI $0.88-1.02$; RD $1.0 \%$ reduction; $95 \%$ CI $2.5 \%$ reduction to $0.4 \%$ increase, moderate certainty) or disability assessed by the Disability Rating Scale (MD, -0.18 points; $95 \% \mathrm{CI}-0.43$ to 0.08 ; moderate certainty). TXA may reduce hematoma expansion on subsequent imaging (RR 0.78; 95\% CI $0.58-1.03$, RD $3.4 \%, 95 \%$ CI $6.5 \%$ reduction to $0.5 \%$ increase, low certainty). Risks of adverse events (all moderate or low certainty) were similar between placebo and TXA.

Conclusion: In patients with acute TBI, TXA probably has no effect on mortality or disability. TXA may decrease hematoma expansion on subsequent imaging; however, this outcome is probably of less importance to patients. The use of TXA probably does not increase the risk of adverse events.

Keywords: Randomized clinical trial, Tranexamic acid, Traumatic brain injury

\section{LO37}

Closed loop communication in the trauma bay: identifying opportunities for team performance improvement through a video review analysis

A. Bhangu, BMSc, L. Notario, MSc, RN, D. Pannell, MD, PhD, W. Thomas-Boaz, MN, RN, C. Freedman, MHI, H. Tien, MD, MSc, A. Nathens, MD, MPH, PhD, L. Da Luz, MD, MSc

Queen's University School of Medicine, Kingston, ON

Introduction: Communication among trauma team members in the trauma bay (TB) is vulnerable to errors. Examples of miscommunication may include misunderstanding and parallel conversations, which can negatively impact patient care. We used the trauma-nontechnical skills (T-NOTECHS) scale to identify communication gaps during assessment and management of patients in the TB and to develop strategies to improve team performance.

Methods: Two reviewers independently assessed non-technical skills of team members for 55 trauma activations at Sunnybrook Health Sciences Centre through video footages. Team performance was measured by the T-NOTECHS scale across five domains: (1) leadership; (2) cooperation and resource management; (3) communication and interaction; (4) assessment and decision making; (5) situation awareness/coping with stress. Secondary outcomes assessed number of callouts (CO), closed loop communications (CLC) and parallel conversations. Team perception of communication was also collected. Results: Injury severity score (ISS) was used as a measure of trauma severity. A case with an ISS score $\geq 16$ was considered to be severe. ISS was $\geq 16$ in $37 \%$ of cases. A Kruskal-Wallis test showed a statistically significant difference between the mean ranks of the five T-NOTECHS domains $(p<0.0001)$. Communication and interaction scored significantly lower $(\mathrm{p}<0.0001$, adjusted using the Bonferroni 
correction) compared with all other domains. More severe cases tended to score a higher median (IQR) number on secondary outcomes, including 6 (5-10) COs, 8.5 (5-13.75) completed CLCs, and 2 (1-3.75) incomplete CLCs. Less severe cases had 4 (2.25-7) COs, 5 (3-8) complete CLCs, and 1 (0-2) incomplete CLC. There were more parallel conversations in less severe cases $2(1-4.75)$ compared with more severe cases $1(0-3)$. From 66 completed questionnaires, there was a slight positive impression on current communication and presence of CLC 1 (0-3); however, there was also a desire for better standardized CLC within trauma team interactions $2(0-4)$.

Conclusion: A lower communication score was identified using TNOTECHS, caused by incomplete CLCs and parallel conversations. Survey results similarly emphasized need for improved CLC. Future initiatives will include CLC training using the pre-briefing checklist, in situ simulations, Trauma Team Video Reviews, and a mandatory educational video on crisis resource management for incoming trainees. Sustainability and cultural change will be addressed in further studies.

Keywords: Non-technical skills, Trauma, Video review

\section{LO38}

Safety and efficiency of the Canadian CT Head Rule in assessing anticoagulated head-injured patients

F. Al-Haimus, MBChB, N. Clayton, RA, K. de Wit, BSc, MBChB, MD, MSc

\section{McMaster University, Hamilton, ON}

Introduction: The proportion of the Canadian population who takes anticoagulant medication is increasing yearly. Current guidelines advise neuroimaging (CT scan) for all head-injured emergency patients who take anticoagulation; however, there has been little research to support this approach. The aim of this study was to evaluate the safety and efficiency of the Canadian CT Head Rule for anticoagulated head-injured emergency department (ED) patients.

Methods: This was a two-hospital health records review. To be included, the patient had to have been prescribed anticoagulant medication prior to ED presentation, to have sustained a blunt head injury within $48 \mathrm{~h}$, be GCS $13-15$ and have had a head CT scan in the ED. To identify consecutive adult patients fulling these criteria we searched the medical records of all patients who had a head CT scan in the ED between June and December 2019. Trained researchers extracted data on participant demographics and the Canadian CT Head Rule criteria, following precise variable definitions. We defined traumatic brain injury (TBI) as the presence of intracranial blood within any compartment, diffuse axonal injury or isolated skull fracture. Participants were followed forward in time by chart review for 30 days and classified as TBI positive or negative. We reported the sensitivity and specificity of the Canadian CT Head Rule among anticoagulated head-injured ED patients for diagnosing TBI.

Results: In total, 5603 patients had a head CT in the ED, from whom, 337 patients met our inclusion criteria. Mean age was 80 and $44 \%$ were male. Twenty-one of these patients were diagnosed with TBI on initial head CT and no patients had a delayed bleed within 30 days. The Canadian CT Head Rule was $100 \%$ sensitive [95\% confidence interval (CI) $81.0-100 \%$ ] and $9.8 \%$ specific (95\% CI 6.9-13.8\%) for TBI. Positive likelihood ratio was 1.1 , negative likelihood ratio was 0 . Conclusion: The Canadian CT Head Rule appears safe for use in anticoagulated head-injured ED patients, but it is not an efficient way to rule out TBI in the population.

Keywords: Anticoagulation, Head trauma, Traumatic brain injury

\section{LO39}

Anxiolysis for laceration repair in children: a survey of paediatric emergency providers in Canada

N. Poonai, MD, MSc, K. Kumar, MD, S. Ali, MD CM, V. Sabhaney, MD, E. Trottier, MD, A. Drendel, MD, A. Shah, MD, M. Bhatt, MD

Western University, London, ON

Introduction: Laceration repair accounts for half of procedures performed in the emergency department. LET (lidocaine, epinephrine, tetracaine) is commonly used as a topical anesthetic but it does little to alleviate the emotional distress, particularly in young children. Intranasal midazolam is commonly used is not consistently effective. Intranasal dexmedetomidine (IND) is a new and potentially effective anxiolytic but clinical uptake is limited by a lack of paediatric data. We sought to understand paediatric emergency providers' willingness, preferences, and perceived barriers to anxiolysis for laceration repair in children to inform the design of a future clinical trial of IND. Methods: This was an online survey of Canadian paediatric emergency providers listed in the Pediatric Emergency Research Canada database. The survey was developed according to published guidelines and featured a vignette of a 3-year-old distressed child requiring laceration repair. It was hosted on the Research Electronic Data Capture platform in both English and French and disseminated from September to December 2020. The primary outcome was the proportion of respondents that would provide anxiolysis. Secondary outcomes included perceived barriers to IND use. Data was reported using summary statistics.

Results: The response rate was 154/225 (68.4\%), of which 97/154 $(63 \%)$ were Royal College accredited paediatric emergency physicians. $128 / 154(83 \%)$ spent $>80 \%$ of their time doing clinical work. $122 / 154(79 \%)$ had $>6$ years independent practice experience and $125 / 154(81 \%)$ performed $>4$ laceration repairs per month. 126/154 $(82 \%)$ believed that $>25 \%$ of children experienced distress that requires physical restraint. 115/154 (75\%) indicated they would provide anxiolysis, mainly as intranasal midazolam $(99 / 115,86 \%)$. 94/154 (61\%) indicated that they would consider IND if evidence suggested it was effective. Common perceived barriers included inadequate personal $(111 / 154,72 \%)$ and nursing $(108 / 154,70 \%)$ experience with IND, and lack of access $(59 / 154,38 \%)$ to IND.

Conclusion: Most paediatric emergency providers believe that laceration repair in a young child is distressing and would provide anxiolysis. Our findings suggest that a clinical trial demonstrating IND's anxiolytic effectiveness for laceration repair in children would have good clinical uptake. However, important barriers such as lack of clinical experience with, and access to IND will need to be addressed to optimize clinical uptake.

Keywords: Anxiolysis, Laceration, Paediatric

\section{LO40}

Transported traumatic cardiac arrest patients managed at a single Ontario lead trauma centre: validation of a diagnostic coding algorithm and descriptive epidemiology

N. Radulovic, BSc, MD, A. Kim, MD, C. Evans, MD, MSc

University of Toronto, Toronto, ON

Introduction: Major injury and trauma continue to be growing concerns for mortality and long-term disability in Canada. Despite the significant implications of traumatic cardiac arrests (TCAs), little progress has been made to better understand the individual variables that can impact prehospital management and outcomes of these patients. Diagnostic and procedural information is routinely captured 
in databases such as the Ontario Trauma Registry (OTR); however, their accuracy in terms of the validity of TCA identification is not well-characterized in current literature. Therefore, this study aimed to evaluate the accuracy of administrative data in identifying the traumatic arrest patient population.

Methods: This retrospective diagnostic accuracy study used data acquired from the OTR between the years 2005-2018. A preliminary list of twelve diagnostic and procedural OTR codes, that were hypothesized to be applicable in TCA identification, was developed. The registry was searched for patients who possessed at least one of these codes and who were managed at a single Ontario lead trauma centre, Kingston Health Sciences Centre (Kingston, Canada), during the study period. Electronic medical records were reviewed to determine whether true TCAs had occurred, and statistical analyses were performed to identify the diagnostic accuracy of individual and combined coding parameters.

Results: One-hundred and thirty patients met the inclusion criteria and were eligible for medical record review. TCAs represented $77 \%$ $(n=100)$ of patients, while $18 \%(n=24)$ sustained a cardiac arrest of non-traumatic etiology and $5 \%(n=6)$ were considered to have cardiac arrests of indeterminate etiology. A prehospital respiratory rate of 0 breaths/min identified true TCA cases with the most favorable combination of sensitivity and specificity (sensitivity $89.7 \%$, 95\% CI 76.4-95.9; specificity $28.9 \%$, 95\% CI $18.3-42.3$ ), along with the combined parameters of required prehospital CPR and a prehospital respiratory rate of 0 breaths/min (sensitivity $91.2 \%, 95 \%$ CI 77.0-97.0; specificity $26.4 \%, 95 \%$ CI $16.4-39.6$ ).

Conclusion: Several individual and combined diagnostic and procedural OTR codes were found to be moderately accurate in identifying traumatic arrest patients. Continued work should aim to expand these data fields and to optimize the accuracy within trauma registries. This may facilitate population-based studies for enhanced prehospital management and outcomes of traumatic arrests.

Keywords: Cardiac arrest, Trauma

\section{LO41}

\section{Venous thromboembolism in patients discharged from the emergency department with ankle fractures: a population-based cohort study}

K. Grewal, MD, MSc, C. Atzema, MSc, R. Sutradhar, PhD, K. Everett, MSc, B. Borgundvaag, MD, PhD, J. Theodoropoulos, MD, D. Horner, MD, MSc, S. McLeod, MSc, PhD, K. de Wit, MD, MSc

\section{University of Toronto/Mount Sinai Hospital, Toronto, ON}

Introduction: Temporary lower limb immobilization may be associated with venous thromboembolism (VTE). However, there is little validated evidence to evaluate risk of VTE when managing patients with ankle fractures in the emergency department (ED), and no Canadian guidelines suggesting who would benefit from thromboprophylaxis in the ED. The objective of this study was to examine the 90-day incidence of VTE among patients discharged from the ED with an ankle fracture requiring immobilization. Secondary objectives were to examine individual risk factors for VTE in this population, and compare the risk of VTE in patients with ankle fractures against a priori selected control groups.

Methods: This was a retrospective analysis of population-based data from 2013 to 2018 of patients $\geq 16$ years discharged from an Ontario ED with a closed ankle fracture requiring temporary immobilization. We estimated 90-day incidence of VTE after ankle fracture. A Cox proportional-hazards model was used to evaluate individual risk factors associated with VTE, censoring at 90 days or death. Patients with an ankle fracture were then propensity score matched on 26 variables to control patients discharged from the ED with other injuries that did not require lower limb immobilization (finger wounds and wrist fractures), to compare the relative hazard of VTE.

Results: 86,081 patients with an ankle fracture were included. Incidence of VTE within 90 days was $1.3 \%$ (1117 patients). Factors associated with VTE were older age (HR 1.18, 95\% CI 1.00-1.39), hospital admission (HR 1.33, 95\% CI 1.05-1.68), surgery (HR 1.58, 95\% CI 1.30-1.93), surgery for ankle fracture (HR 1.80, 95\% CI 1.48-2.20), and VTE history (HR 5.18, 95\% CI 4.33-6.20). In the matched cohort, patients with an ankle fracture had an increased hazard of VTE compared to matched controls with a finger wound (HR 6.31, 95\% CI 5.30-7.52) and wrist fracture (HR 5.68, 95\% CI 4.71-6.85). Several sensitivity analyses were conducted and these results did not change substantively.

Conclusion: The 90-day incidence of VTE among patients discharged from the ED with an ankle fracture requiring immobilization was $1.3 \%$, which is higher than the $0.1-0.2 \%$ risk per year of VTE quoted in the general population. Patients with an ankle fracture had a five to sixfold increased hazard of VTE compared to matched controls with a finger wound or wrist fracture. Future studies examining the role of selective thromboprophylaxis in high risk patients with ankle fractures discharged from ED are needed.

Keywords: Ankle fracture, Venous thromboembolism

\section{LO42}

The implementation and efficacy of Iloprost in management of frostbite injuries in a large urban centre

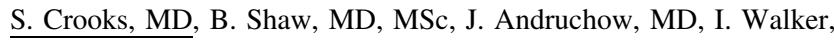
MD

University of Calgary, Calgary, $\mathrm{AB}$

Introduction: In Canada's northern climate, frostbite is a common reason for presentation to the Emergency Department. Traditionally, high grade frostbite was managed with re-warming, wound care and delayed amputation. One emerging therapy in the literature for frostbite management is the vasodilator Iloprost. Iloprost is a synthetic prostaglandin analogue used for pulmonary hypertension. In 2019, Foothills Medical Centre received special approval to use Iloprost off label for severe frostbite. This was based on a handful of small, but important studies that showed Iloprost resulted in a significantly lower amputation rate. We assessed the efficacy and safety of an Iloprost protocol in a large urban centre on frostbite patients with a wide spectrum of co-morbid disease and social factors contributing to their frostbite and prognosis.

Methods: A retrospective chart review of 90 consecutive patients who presented to Calgary Emergency Departments from April 2017 to April 2020 with grade 2-4 frostbite injuries was performed. Patients from 2019 onward were managed using an Iloprost protocol developed in Whitehorse, Yukon. The primary outcome was the number of patients who underwent digital amputations for each grade of frostbite in patients treated with Iloprost vs. without Iloprost. The secondary outcome was to confirm the safety profile of Iloprost and determine the optimal disposition of frostbite patients.

Results: 26 patients were treated with Iloprost, compared to 64 patients who received usual care without Iloprost. In both groups, over $30 \%$ of the patients were homeless and over $50 \%$ were intoxicated with drugs or alcohol at the time of injury. In grade 2-4 frostbite treated with Iloprost, the amputation rates were $0 \%, 42 \%$ and $80 \%$ respectively. This was compared to the non-Iloprost group where the grade $2-4$ amputation rates were $0 \%, 71 \%(\mathrm{p}=0.044)$ and $100 \%$ $(\mathrm{p}=0.159)$ respectively. There were no adverse events associated with Iloprost other than mild headache in $15 \%$ of patients.

Conclusion: This is the largest study to date on the efficacy of Iloprost in severe frostbite. Previous studies have included primarily 
healthy patients with cold injuries from wilderness pursuits. In this novel urban population, Iloprost continues to be a safe effective therapy for lowering amputation rates. We believe this study demonstrates the benefit of implementing a frostbite treatment pathway for patients presenting to urban Canadian Emergency Departments.

Keywords: Cold injury, Frostbite, Iloprost

\section{LO43}

Silver lining to a lockdown? Impact of the early COVID-19 pandemic on the incidence of traumatic injuries presenting to adult emergency departments (EDs): a multicentre analysis

D. Chisholm, BSc, A. Couperthwaite, MSc, E. Lang, MD CM

University of Alberta, Edmonton, AB

Introduction: On March 11th 2020, the Alberta government instituted public health orders aimed at curbing COVID-19 spread. While certain jurisdictions experienced surges of COVID-19 patients, many Canadian EDs experienced decreased patient volumes. Traumatic injuries are the leading cause of death for those $<40$ years old in Alberta and are a burden on ED resources. The primary objective was to investigate the impact of the early stages of the COVID-19 pandemic on the incidence of traumatic injuries presenting adult EDs in Calgary. The secondary objectives were to describe the impact of the early pandemic on injury type and mechanism of injury (MOI).

Methods: A retrospective cohort design using administrative data was used. Patients 18 years of age or older presenting to one of four Calgary adult EDs during the study period with a traumatic injury were included. The study period was between March 15th to April 26th, 2019 (control cohort) and March 15th to April 26th, 2020 (COVID-19 cohort). A univariate incidence rate ratio (IRR) was calculated to determine if there was any difference between the incidence of traumatic injury presentations in 2019 and 2020. Descriptive statistics were used to compare age, sex, triage score, method of arrival, and disposition status between both groups. The 15 most common MOI based on ICD-10 codes and presenting complaints were analyzed.

Results: There were 2944 injury presentations during the 2020 COVID cohort and 4749 during the 2019 cohort (crude IRR 0.62; 95\% CI 0.59-0.65). Baseline characteristics were similar between both groups. There were 7 ICU and 518 ward admissions for trauma in 2020, compared to 13 ICU admissions and 627 ward admissions in 2019. A greater proportion of patients in the COVID cohort were admitted to hospital, with $18 \%$ admitted to hospital in 2020 versus $13 \%$ in 2019 . The most common presenting complaints and MOI were similar between both cohorts, with the top three presenting complaints being lower, and upper, extremity injury and laceration/ puncture. While there was a $58 \%$ reduction in motor vehicle collision related complaints in 2020, rates of penetrating major trauma and burns in 2020 were similar to 2019.

Conclusion: There was a dramatic $38 \%$ reduction in the incidence of traumatic injuries presenting to Calgary adult EDs during the early COVD-19 pandemic. While the absolute number of most presentation types were reduced, notably there was no reduction in the incidence of penetrating trauma or burns.

Keywords: COVID-19, Crowding, Trauma

\section{LO44}

Interventions to reduce burnout in the emergency department: a systematic review
A. Laviolette, BSc, Z. Ma, BHSc, H. Kearney, MSc, D. Malhotra, MSc, C. Davies-Schinkel, MSc, M. Luterman, MD, MSc, S. Upadhye, $\mathrm{MD}, \mathrm{MSc}$

\section{McMaster University, St. Catharines, ON}

Introduction: Burnout is a syndrome characterized by emotional exhaustion, physical fatigue, and cognitive weariness, and is consistently reported at higher rates in Emergency Medicine (EM) physicians compared to other medical specialties. Published studies examining interventions targeting burnout among physicians and nurses are scarce. This systematic review aimed to identify interventions that reduce burnout among EM physicians, residents, and nurses.

Methods: A systematic review was conducted using PRISMA and Cochrane Collaboration guidelines. A literature search was performed using MEDLINE Ovid, PsycInfo, CINAHL, Web of Science, CCTR, and EMBASE. Primary literature published in English that investigated interventions on burnout in ED staff using validated assessment tools were included. Four reviewers completed titles and abstracts screening, full text screening, and data abstraction in duplicate. Two reviewers completed a quality assessment using the Cochrane risk of bias tool for randomized controlled trials or the Risk of Bias for NonRandomized Studies (ROBINS-I) for non-randomized studies.

Results: The search strategy identified 2357 potential studies and 7 were eligible for inclusion. Most studies measured burnout using the Maslach Burnout Inventory, while others used the Copenhagen Burnout Inventory, or the Professional Quality of Life tool. Studied interventions were heterogeneous and included mindfulness training, group seminars, didactic sessions, multimedia resources, and attention-based training. Included studies showed variation in duration, ranging from 2 weeks to 6 months, and interventional approach to mitigating EM staff burnout. Physicians and nurses benefitted from interventions that addressed themes of mindfulness, reflective practice, and problem-solving, and were longer in duration. One study highlighted that EM residents found these intervention programs to be time-consuming and burdensome-and may contribute to their level of self-reported burnout. All included studies were rated as serious to critical for risk of bias and all studies recorded high rates of attrition.

Conclusion: This systematic review examined interventions targeting burnout in EM staff, and showed that different interventions had variable degrees of benefit depending on the target population. These results can be used to create and implement targeted interventions to different EM providers, and demonstrate the need for more robust research studies aimed at tailored interventions to mitigate burnout.

Keywords: Burnout, Emergency medicine, Systematic review

\section{LO45}

Patient characteristics and outcomes associated with cancer diagnosis in the emergency department: a systematic review

S. Kang, BHSc, MD, S. McLeod, PhD, C. Walsh, MA, K. Grewal, MD, MSc

University of Toronto, Toronto, ON

Introduction: A significant proportion of patients are initially diagnosed with cancer in the emergency department (ED). The objective of this systematic review was to compare patient characteristics (age, sex, socioeconomic status) and outcomes (stage of cancer, mortality/survival) for patients diagnosed with cancer in the ED compared to those diagnosed elsewhere.

Methods: Electronic searches of Medline and EMBASE were conducted and reference lists were hand-searched. Studies 
comparing adult patients diagnosed with any type of cancer in the ED (ED diagnosis) to patients diagnosed elsewhere (non-ED diagnosis) were included. Two reviewers independently screened titles and abstracts and extracted data. The quality of the included studies was independently assessed in duplicate using the Newcastle-Ottawa scale for cohort studies. A meta-analysis was not conducted due to significant between-study heterogeneity. Data pertaining to patient characteristics and outcomes were summarized and synthesized narratively.

Results: 20 studies were included (78,741 in the ED diagnosis group, and 1,340,956 in the non-ED group). Ten studies were assessed to be of good quality, one study was fair quality, and nine studies were poor quality. The majority of studies (9/15 studies) that included patient age showed a statistically significant association between older age and an ED diagnosis of cancer (odds ratios (ORs) ranged from 1.3 to 11.1). In 6 of 13 studies that reported patient sex, female sex was associated with an ED diagnosis of cancer. The remaining studies showed no association between patient sex and ED diagnosis. There was no consistent association between measures of socioeconomic status and ED diagnosis between studies. All 12 studies that reported stage of cancer at diagnosis reported a higher stage in patients with an ED diagnosis of cancer (ORs of advanced stage with an ED diagnosis ranged from 1.1 to 14.7$)$. The majority of studies (8/9 studies) that examined mortality reported increased mortality among patients with an ED diagnosis of cancer (hazard ratios of mortality with an ED diagnosis ranged from 1.5 to 4.1 ).

Conclusion: Patients with an ED diagnosis of cancer were older, had a higher stage of cancer at diagnosis and increased mortality compared to patients who were diagnosed elsewhere. Increased support, resources for patient navigation and timely access to cancer care are required for patients who are newly diagnosed with cancer in the ED. Keywords: Cancer, Diagnosis, Emergency department

\section{LO46}

\section{Effective pain control for corneal abrasions in the emergency} department: a meta-analysis

C. Yu, BHSc, A. Kirubarajan, MSc, M. Yau, BHSc,

D. Armstrong, BSc, D. Johnson, MD

\section{Kingston Health Sciences Centre, Kingston, ON}

Introduction: Pain management for corneal abrasions differs amongst emergency physicians, thus this meta-analysis was conducted to evaluate the efficacy and safety of six topical pain therapies: topical anesthetics, non-steroidal anti-inflammatory drugs (NSAIDs), cycloplegics, steroids, pressure patching, and bandage contact lens (BCL).

Methods: The protocol followed PRISMA guidelines and was published on PROSPERO. MEDLINE, EMBASE, CENTRAL, and Web of Science were searched to May 2020. Primary studies comparing topical pain therapies to other therapy or control were included. Primary outcomes included percentage of abrasions healed at 24,48 , and $72 \mathrm{~h}$. Secondary outcomes included pain control at 24 and $48 \mathrm{~h}$, use of oral analgesia, and prevalence of complications. Risk of bias was assessed using the Cochrane guidelines and the MINORS criteria. Quality of evidence for estimates was assessed by the Grading of Recommendations, Assessment, Development and Evaluation (GRADE) tool. Subgroup analysis was conducted for heterogeneity.

Results: Overall, 30 RCTs and 2 cohort studies (4056 patients) were analyzed. Risk of bias was low for most studies. Only topical NSAIDs showed significantly reduced pain scores at $24 \mathrm{~h}[\mathrm{RR}-0.69,95 \% \mathrm{CI}$ $(-0.98,-0.41)]$ and $48 \mathrm{~h}[\mathrm{RR}-0.56,95 \% \mathrm{CI}(-1.02,-0.10)]$, and lower oral analgesia use $[\mathrm{RR} 0.47,95 \% \mathrm{CI}(0.33,0.66)]$ compared to control. Topical anesthetics, cycloplegics, patching, and BCL did not result in significant reduction in pain scores or oral analgesia use. No studies examined topical steroids. No intervention resulted in significant healing delays or higher complication rates compared to controls. GRADE certainty of evidence ranged from low to moderate for outcomes.

Conclusion: Topical NSAIDs significantly reduced pain from corneal abrasions in the first $48 \mathrm{~h}$ and led to lower oral analgesia use without increased rates of complications or delays in healing. Evidence does not support the use of topical anesthetics, cycloplegics, steroids, pressure patching, or BCL for pain control in corneal abrasions.

Keywords: corneal abrasions, topical analgesia

\section{LO47}

Drug treatments for COVID-19: a living systematic review and network meta-analysis

S. McLeod, MSc, PhD, R. Siemieniuk, MD, PhD, G. Guyatt, MD, MSc, OC, D. Zeraatkar, PhD, J. Bartoszko, MSc, E. Kum, MSc, L. Ge, PhD, A. Izcovich, MD, PhD, A. Khamis, MD, MPH, R. Brignardello-Petersen, $\mathrm{PhD}$

SREMI, Sinai Health, University of Toronto, Toronto, ON

Introduction: As of December 2020, nearly 70 million people have been infected with severe acute respiratory syndrome coronavirus virus 2 (SARS-CoV-2), the virus responsible for coronavirus disease 2019 (COVID-19). The objective of this living systematic review and network meta-analysis (NMA) is to compare the effectiveness of drug treatments for COVID-19.

Methods: Daily searches are conducted using the World Health Organization COVID-19 database, a comprehensive multilingual source of global literature including 25 bibliographic and grey literature sources. Randomised controlled trials involving patients with suspected or confirmed COVID-19 comparing the use of drugs to other drugs, placebo or standard care are included. Pairs of reviewers independently screen potentially eligible articles, extract data and assess risk of bias $(\mathrm{RoB})$ on included trials using a modified Cochrane RoB 2.0 tool. Using a Bayesian framework, we conducted a NMA of drug treatments for COVID-19. The certainty of evidence was assessed using GRADE approach for NMA using a minimally contextualised approach.

Results: 85 trials enrolling 41,669 patients were included. Compared with standard care, corticosteroids probably reduce death (odds ratio (OR) 0.85 ; 95\% CI 0.71-1.01; risk difference 17 fewer per 1000 patients, 95\% CI 34 fewer to 1 more, moderate certainty), mechanical ventilation (OR $0.72 ; 0.50-1.01 ; 29$ fewer per 1000 patients, 54 fewer to 1 more, moderate certainty), and days free from mechanical ventilation (2.6 fewer, 0.2-5.0 fewer, moderate certainty). The impact of remdesivir on mortality, mechanical ventilation, length of hospital stay and duration of symptoms is uncertain, but probably does not increase adverse effects leading to drug discontinuation (OR 1.00; 0.37-3.83; 0 more per 1000, 9 fewer to 40 more, moderate certainty). Azithromycin, hydroxychloroquine, lopinavir/ritonavir, interferon-beta, and tocilizumab may not reduce risk of death or have an effect on any other patient-important outcome. The certainty in effects for all other interventions was low or very low.

Conclusion: Corticosteroids may reduce mortality, mechanical ventilation and ventilator-free days in patients with severe COVID19. Whether or not remdesivir confers any patient-important benefit remains uncertain. Azithromycin, hydroxychloroquine, lopinavir/ritonavir, interferon-beta, and tocilizumab may not reduce risk of death or have an effect on any other patient-important outcome. Keywords: COVID-19, Living network meta-analysis, Treatment 


\section{LO48}

Impact of COVID-19 protocols on emergent intubation performance in the emergency department, intensive care unit, and during ward code-blues in an urban tertiary-care centre

J. Fernandes, BSc, MPA, C. Renschler, MD, K. Dullemond, MD, D. Griesdale, MD, F. Scheuermeyer, MD, J. Yoo, MD

University of British Columbia, Vancouver, BC

Introduction: The COVID-19 pandemic has forced most hospitals to implement protocols to mitigate risk of disease transmission to health care providers during endotracheal intubation (ETI). The logistics of performing ETI differ across critical-care areas within a hospital. We evaluated the impact of COVID-19 protocols on ETI performance and complications in the emergency department (ED), intensive care unit (ICU), and during Ward Code Blues (WCBs) and describe how ETI practices have changed.

Methods: We performed a retrospective observational study of patients intubated at an urban tertiary-care hospital in Vancouver, BC using standardized registry data. Pre-COVID, defined as ETIs performed 1 year before the pandemic (Mar 1, 2019-Feb 29, 2020), was compared with ETIs performed post-COVID, defined as a 6 month period during the pandemic (May 1-Oct 31, 2020). The transition period (Mar 1-Apr 30, 2020) was excluded, as hospital logistics and protocols were rapidly changing and likely not reflective of performance during the pandemic. The primary outcome was first-attempt success in the absence of complications (FASAC). Secondary outcomes were first-attempt success (FAS) and complications. We analyzed using descriptive techniques.

Results: Pre-COVID 564 intubations $(\mathrm{ED}=310, \mathrm{ICU}=183, \mathrm{CB}=$ 71) were performed, and post-COVID 387 intubations $(E D=212$, $\mathrm{ICU}=121, \mathrm{CB}=54)$ were performed. FASAC rose in the ED (81.9$89.2 \%, p=0.05)$ and ICU $(68.9-78.5 \%, p=0.20)$, while it dropped during WCBs $(81.7-74.1 \%, \mathrm{p}=0.42)$. FAS increased in the ED (87.4-93.9\%, $\mathrm{p}=0.01)$ and ICU $(78.7-87.6 \%, \mathrm{p}=0.04)$ and fell during WCBs $(91.5-83.3 \%, \mathrm{p}=0.18)$. Complication rates decreased in the ED $(10.6-7.1 \%, \mathrm{p}=0.15)$ and ICU $(19.1-13.2 \%, \mathrm{p}=0.17)$ but increased in WCBs $(12.7-16.7 \%, \mathrm{p}=0.54)$. The most frequent complications in both pre- and post-COVID were desaturation after induction and aspiration. In the ED, the proportion of EM operators increased from 79.7 to $87.3 \%$, while Anesthesia operators increased in both the ICU (13.1-21.5\%) and WCBs (46.5-53.7\%). All three locations had increases in staff physician intubations (ED: $+12.4 \%$, ICU: $+9.8 \%$, WCB: $+12.8 \%)$ and the use of video laryngoscopy (VL) (ED: $+14.6 \%$, ICU: $+23.6 \%$, WCB: $+13.4 \%$ )

Conclusion: ETI performance in the ED and ICU have improved but WCB performance may have been negatively impacted by the challenges of the COVID-19 pandemic. ETIs were more frequently performed by senior airway operators with $\mathrm{VL}$ as the technique of choice in all three locations.

Keywords: Airway, COVID-19, Intubation

\section{LO49}

The virtual delivery of a national rural medical education and insitu simulation curriculum

W. Lam, MD, F. Gilic, MD, J. Guscott, MD, M. Tromp, MD, J. Perry, MD, MSc, M. O'Brien, MD, W. Cheung, MD, MMed

Department of Emergency Medicine, University of Ottawa, Ottawa, ON

Innovation concept: Delivery of simulation training to rural emergency departments (EDs) has traditionally relied upon costly physical relocation of educators or learners, which has become unfeasible in the COVID-19 pandemic environment. We sought to develop and evaluate the educational utility of a novel curricular framework to facilitate the virtual delivery of in-situ simulation.

Methods: We collaborated with the Society of Rural Physicians of Canada to recruit 14 rural EDs across Canada, with a cumulative annual ED census of 322,004, and perform a needs assessment among 16 experienced rural educators using a nominal group technique to inform the educational framework. We then used the Kern Six Step approach to further define the learning objectives for each community while considering regional practice guidelines and regulations. Throughout the delivery of the virtual curriculum, surveys were distributed after each curricular phase to facilitate Kirkpatrick program evaluation. Each survey item was rated using a five-point Likert scale of agreement.

Curriculum, tool, or material: We developed a novel three-phase curricular framework rooted in the levels of processing model. Phase one consists of four didactic videos. Phase two consists of five spotthe-difference simulation videos demonstrating various medical errors; learners are instructed to debrief performance using the Plus/ Delta framework. Phase three consists of in-situ simulation facilitated virtually by experienced interdisciplinary simulation educators. All participants $(\mathrm{n}=15)$ perceived virtual in-situ simulation "as good as" $(n=11)$ or "better than" $(n=4)$ traditional in-person simulation. Participants agreed that the curriculum facilitated knowledge acquisition (median 5, IQR 1), was a positive learning experience (median 5 , IQR 1), was a satisfactory use of time (median 5, IQR 0 ), and would be recommended to colleagues (median 5, IQR 0). Both spotthe-difference (median 5, IQR 1) and virtual debriefing (median 4.5, IQR 1) were rated psychologically safe.

Conclusion: We present a novel framework for the virtual delivery of in-situ simulation that is independent of learner-educator geography and decreases financial barriers to access for learners. Our curriculum provides a simulation experience for rural ED physicians that is rated comparable to or better than traditional in-person simulation and promotes the accessibility of in-situ simulation programs in rural and remote EDs across Canada.

Keywords: Innovations in EM education, Simulation, Virtual

\section{LO50}

HiQuiPs-health informatics quality improvement patient safety: a novel free open access medical education resource

A. Taher, MD, MPH, L. Chartier, MD CM, MPH, S. Mondoux, MD, MSc, M. Hacker Teper, BA, MSc, M. Hewitt, MD, D. Lee, BSc, C. Parpia, BHSc, L. Pozzobon, BHSc, MSc, S. Campbell, BSc, E. Mason, MD

University of Toronto, Toronto, ON

Innovation Concept: The quality improvement (QI) paradigm has been increasingly utilized in healthcare. This is paralleled with an increase in QI, patient safety (PS) and health informatics (HI) educational programs. Antecedent work revealed knowledge gaps in foundational QI/PS/HI concepts for learners and interprofessional team members, creating barriers to successful patient care improvements. We developed a FOAMed online series called HiQuiPsHealth informatics, Quality improvement \& Patient safety-to address this gap.

Methods: We used the Kern Six-Step Curriculum Development Model. Based on our identified gap, we carried out a needs assessment through stakeholder engagement with HI/QI/PS experts across the country and a review of available content. We identified main goals of: (1) fostering a basic understanding of the three content areas for EM providers; (2) ensuring country-wide content expert 
contribution; and (3) promoting broad engagement of interprofessional uptake. Our strategy involved online blog posts on the CanadiEM platform consisting of foundational topics, with crowdsourced feedback to inform themes for future material.

Curriculum, tool, or material: HiQuiPs launched in July 2018 and as of December 2020 has disseminated 33 posts written by faculty, learners, and allied health from 18 institutions across 5 provinces. Posts also include collaborations with prominent national organizations (e.g., Choosing Wisely Canada) and special posts on relevant topics, such as the COVID-19 pandemic. Posts are approximately 1000 words and framed in a practical format with a clinical vignette to assist in knowledge translation. As of December, the series has received 51,573 page-views with 24,633 unique views. HiQuiPs initially addressed foundational concepts within the core material, before expanding to advanced topics to be relevant and useful to the series' diverse audience. Similarly, our focus on the EM setting to anchor practical examples is being transitioned to other departments and healthcare settings beyond the hospital to appeal to our growing readership.

Conclusion: HiQuiPs is a novel FOAMed resource disseminating foundational topics in HI, QI and PS that has seen increased uptake in readership and engagement by EM providers. Future plans include improving knowledge translation with alternative formats such as infographics, refining material based on feedback, and increasing evaluation in order to higher-quality, relevant content.

Keywords: Innovations in EM education, Medical education, Quality improvement

\section{LO51}

Developing the Virtual Resus Room: usability, accessibility, and applicability of a virtual simulation alternative for teaching and learning

S. Foohey, BHSc, MD, A. Nagji, BHSc, MD, Y. Yilmaz, MSc, PhD, M. Sibbald, MD, PhD, S. Monteiro, PhD, T. Chan, MD, MHPE

University of Toronto, Toronto, ON

Innovation concept: Physical distancing restrictions resulting from the COVID-19 pandemic led to the transition from in-person simulation teaching to online alternatives. It was challenging to find an open-access, cost-limited, interactive, virtual solution that provided a meaningful substitute for the sim lab. The Virtual Resus Room (VRR) was developed by the lead author to provide a collaborative, openaccess online environment in which learners could rehearse their crisis resource management skills and apply their medical knowledge using simulated patient cases.

Methods: The VRR uses a combination of Google Slides and Zoom. Learners complete tasks collaboratively by making synchronous edits to their shared Google Slide "room", which consists of a patient silhouette surrounded by moveable images of resuscitation equipment. Learners and facilitators communicate with each other using Zoom or an equivalent program. The VRR interface was initially improved through multiple phases of peer review by residents and facilitators. A formal program evaluation study was then conducted. Curriculum, tool, or material: The VRR was used to run two cases for McMaster clerkship students. Learners $(n=46)$ and facilitators $(n=11)$ then completed the program evaluation surveys. Facilitators and learners reported high scores for usability, acceptability, and applicability. Medical students demonstrated an improvement from their pre-test scores $(\mathrm{M}=71.17 \pm 15.77) \quad$ to post test $(\mathrm{M}=89.06 \pm 9.56), \mathrm{t}(34)=7.28, \mathrm{p}<0.001$ with a large effect size Cohen's $d=1.23$. Thematic analysis of participant responses identified two perceived learning outcomes: content learning and communication skill development. The total time spent facilitating virtual simulation using the VRR $(119 \pm 36)$ was significantly lower than time spent leading in-person simulation $(\mathrm{M}=181 \pm 58)$, $\mathrm{U}=20.50, \mathrm{p}<0.008$.

Conclusion: The VRR interface offers an interactive online simulation environment that has been shown to be well accepted by medical students and facilitators. Limitations of the tool include the need for specific resources (reliable internet) and limited fidelity for specific motor skills. The VRR has also been used to provide education to residents, with plans in progress to use it for interprofessional education and continuing professional development. The VRR is an open-access resource that can easily be adapted to a multitude of cases, learners, and environments to provide an effective simulation experience.

Keywords: Distance learning, Innovations in EM education, Simulation

\section{LO52}

Troponin for ED syncope evaluation: derivation and validation of the Canadian Syncope Risk Score ultra-low-risk criteria

V. Thiruganasambandamoorthy, MD, MSc, E. Mercier, MD, MSc, A. McRae, MD, PhD, M. Nemnom, MSc, B. Rowe, MD, MSc, N. Le Sage, MD, PhD, M. Hegdekar, MD, A. Finlayson, MD, C. Leafloor, MD, P. Huang, MD, M. Sivilotti, MD, MSc, M. Taljaard, PhD, J. Yan, MD, MSc

Department of Emergency Medicine, University of Ottawa, Ottawa, ON

Introduction: Cardiac troponin (cTn) aids in identification of serious conditions [e.g. myocardial infarction (MI)] during emergency department (ED) syncope evaluation and is an independent risk factor for 30-day serious adverse events (SAE) after ED disposition. Which patients require cTn testing is unknown and testing all patients will unnecessarily prolong ED stay. Our objectives were to derive and validate the Canadian Syncope Risk Score (CSRS) ultra-low-risk (ULR) criteria for identifying ED syncope patients with abnormal cTn values.

Methods: We previously conducted two large prospective studies and enrolled adults with syncope at 11 EDs from Sep 2010 to Jul 2019. Baseline/event characteristics, ED investigations (ECG, cTn performed at physician's discretion) with results, and 30-day adjudicated SAEs including those identified in ED were collected. Outcome is abnormal cTn, defined as $>99$ th percentile for normal population. SAEs include MI, arrhythmia, structural heart disease, pulmonary embolism, hemorrhage, or death. We used CSRS predictors (vasovagal predisposition, heart disease, ECG abnormalities, triage systolic BP, clinical impression: cardiac/non-cardiac syncope) and age for logistic regression.

Results: 8782 patients (mean age 55.0 years, $54.5 \%$ female; 622 with 30-day SAE) were enrolled; cTn was measured in $4882(55.6 \%)$ patients (cTn group) and was abnormal in $543(11.1 \%)$ patients. We used data to predict abnormal cTn from 2450 and 2432 patients in cTn group for derivation and validation, respectively. The final model included age $\leq 50$ years, no heart disease, normal ECG (QRS axis $\geq-30^{\circ}$ to $\leq 100^{\circ}$, QRS $\leq 130 \mathrm{msec}$, $\mathrm{cQT} \leq 480 \mathrm{msec}$ ) and clinical diagnosis of non-cardiac syncope. In the pooled data, presence of all criteria had a sensitivity $96.5 \%$ (95\% CI 94.6, 97.9), specificity $20.5 \%$ (95\% CI 19.3, 21.7); and c-statistic 0.75 (95\% CI 0.73, 0.77) for abnormal cTn. In the cTn group, ULR criteria identified $97 \%$ (514/530) patients with 30-day SAE [16 misclassified patients were: 11 with SAE in ED (6 arrhythmia, 5 non-cardiac) and 5 after ED disposition ( 1 cardiac)]. Of the 3900 patients in the non-cTn group [92 (2.4\%) patients with 30-day SAE], the criteria application would lead to cTn testing in $1497(38.4 \%)$ patients [83 (5.5\%) patients with 30day SAE: 6 deaths, 50 cardiac and 27 non-cardiac SAE]. 
Conclusion: The CSRS ULR criteria can guide troponin testing decisions for ED syncope, expedite disposition decisions and overall optimize resource utilization.

Keywords: Emergency department, Syncope, Troponin

\section{LO53}

Comparing the frequency of ST-elevation myocardial infarction bypasses and their associated short term outcomes during and before the COVID-19 pandemic

L. Yau, MD, BMSc, B. Vujcic, BSc, M. Davis, MD, MSc

Western University, London, ON

Introduction: In addition to the best efforts to contain the COVID-19 pandemic, it is also important to understand the impact that the pandemic has had on patients who suffer from other acute illnesses such as ST elevation myocardial infarction (STEMI) especially in the prehospital setting. The purpose of our study is to examine the frequency and short-term outcomes of patients transported by Emergency Medical Services (EMS) to a tertiary care centre under STEMI bypass protocol before and during the initial three reopening stages of the COVID-19 lockdown in Ontario.

Methods: This retrospective cohort study enrolled adult STEMI bypass patients who were transported by EMS to a tertiary care centre equipped with a cardiac catheterization lab. The pre-COVID cohort consisted of STEMI bypass patients from March 11 to July 17 in 2017, 2018, and 2019. The COVID cohort consisted of STEMI bypass patients from March 11 to July 17 in 2020 . We collected standardized variables at EMS presentations from history, hospital length of stay (LOS), ICU admission rates, final discharge diagnosis as well as inhospital and out-hospital 30 day mortality rates. Chi-square test and two tailed $t$ test were used for analysis to compare the COVID cohort to each pre-COVID cohort.

Results: In total, 156 patients were enrolled (mean age 64.0 years, $35.6 \%$ female, $27.5 \%$ with known cardiac history). The total number of STEMI bypasses in 2017, 2018, 2019, 2020 were 38, 37, 34, and 41 respectively. There was no statistical difference when examining hospital LOS, ICU admission rates, in hospital and out of hospital 30 day mortality rates between the COVID and pre-COVID cohorts. There was a greater proportion of patients being discharged with a diagnosis of STEMI in the COVID cohort compared to the 2017 and 2019 pre-COVID cohorts $(\mathrm{p}=0.02 ; 0.01)$ but not when compared to the 2018 pre-COVID cohort $(\mathrm{p}=0.11)$.

Conclusion: During the initial re-opening stages of the COVID-19 pandemic, there was a similar total number of STEMI bypasses when compared to previous 3 years. There also appears to be no significant differences in the short term outcomes for these patients. Our study may serve as a potential model to help other centres examine how the COVID-19 pandemic has affected STEMI care, especially in the prehospital setting.

Keywords: COVID-19, Prehospital, STEMI

\section{$\mathbf{L O 5 4}$}

Evaluation of adverse events associated with electrical cardioversion in patients with acute atrial fibrillation and atrial flutter

I. Stiell, MD, MSc, M. Nemnom, MSc, E. Brown, BA, D. Eagles, MD, MSc, RAFF Study Authors

Department of Emergency Medicine, University of Ottawa, Ottawa, ON
Introduction: While the effectiveness and safety of pharmacological cardioversion has been well studied, little research has been done on the safety of electrical cardioversion (ECV) in the ED for patients with acute atrial fibrillation and atrial flutter (AAFF). The overall goal of this study was to evaluate the effectiveness and safety of ECV for AAFF patients in the ED.

Methods: This was a secondary analysis of existing data sets of four previous multicentre AAFF studies conducted by the principal investigator at 30 large academic and community EDs in 8 Canadian provinces. We included all patients enrolled in the previous four studies with AAFF who received attempts at ECV. These adult patients presented to the ED within $48 \mathrm{~h}$ of onset of the arrhythmia or within 7 days of onset if they had been adequately anticoagulated. For the current study, we reviewed all records manually to abstract data onto a standardized form on the use and success of ECV, serious adverse events (death or life-threatening), other adverse events requiring treatment and adverse events not requiring treatment. Research ethics approval was granted. We conducted simple descriptive and univariate statistics.

Results: Of 1752 database cases, we enrolled 834 (47.6\%) patients who had undergone attempted ECV and they were mean age 60.2 years, male $66.8 \%$, had prior AAFF $73.2 \%$, past heart failure $2.9 \%$, past coronary artery disease $1.2 \%$, admitted to hospital $5.0 \%$, received antiarrhythmic drug prior to ECV $44.0 \%$. For ECV, the overall success was $88.0 \%$ with $17.1 \%$ undergoing $>1$ shock, $92.6 \%$ performed by ED physician, $95.1 \%$ sedation given by ED physician, and $92.9 \%$ propofol used. Of these cases $20.0 \%$ suffered adverse events: (a) serious/life-threatening $0.2 \%$ (bradycardia $0.1 \%$, ventricular tachycardia $0.1 \%$ ); (b) requiring treatment $16.8 \%$ (airway maneuvers $12.2 \%$, hypoxia $4.5 \%$, hypotension $3.2 \%$, vomiting $1.1 \%$, aspiration $0.2 \%$ ); (c) not requiring treatment $4.7 \%$ (new conduction problems $2.2 \%$, dysrhythmia $2.1 \%$, pain $0.6 \%$, agitation $0.5 \%$, skin burn $0.1 \%$ )

Conclusion: This is the largest reported evaluation of the efficacy and safety of ECV for AAFF in the ED and found overall $88 \%$ success. While serious adverse events were rare, there were a surprising proportion of cases with adverse events requiring intervention as well as a smaller proportion that did not require treatment. These data should assist physicians in shared decision-making discussions with patients when choosing between pharmacological and electrical cardioversion of AAFF in the ED.

Keywords: Adverse events, Atrial fibrillation, Cardioversion

\section{LO55}

Is high-sensitivity troponin better than conventional troponin for prognostication of ED patients with syncope?

V. Thiruganasambandamoorthy, MD, MSc, A. McRae, MD, C. Leafloor, MD, J. Yan, MD, E. Mercier, MD, N. Le Sage, MD, B. Rowe, MD, M. Hegdekar, MD, R. Booth, MSc, PhD, P. Huang, MD, M. Mukarram, MBBS, A. Ishimwe, BN, M. Nemnom, MSc, M. Taljaard, PhD, M. Sivilotti, MD

Department of Emergency Medicine, University of Ottawa, Ottawa, ON

Introduction: Recent studies identify elevated high-sensitivity cardiac troponin (hs-cTn) as an independent risk factor for 30-day serious adverse event (SAE) among emergency department (ED) patients with syncope; however, many EDs still use convention cTn assay. Our objective was to compare the prognostic abilities of conventional and hs cTn assays (at the 99th percentile threshold for normal population) when combined with other Canadian Syncope Risk Score (CSRS) items to predict 30-day SAE after ED disposition. 
Methods: We used pre-existing data from two large prospective studies that enrolled adults with syncope at 11 Canadian EDs from Sept 2010 to July 2019. Baseline characteristics, CSRS predictorsclinical, ECG and cTn levels (conventional vs. hs), and 30-day adjudicated SAE (arrhythmia, myocardial infarction, structural heart disease, pulmonary embolism, hemorrhage, death) after ED disposition were collected. We report the likelihood ratios (LR) and unadjusted odds ratio (OR) for 30-day SAE; adjust for clinical and ECG CSRS predictors using regression to report adjusted OR and compare the area under the curve (AUC) between the two groups.

Results: 4637 patients (mean age 64.2 years; $51.9 \%$ male) were enrolled. 3554 patients $(76.6 \%)$ had cTn levels measured by conventional assay and $1083(23.4 \%)$ patients by hs assay; $4.6 \%$ and $26.7 \%$ were above the thresholds, respectively. Overall, 274 patients (5.9\%) experienced 30-day SAE: $0.5 \%$ death, $3.9 \%$ arrhythmia, $0.7 \%$ non-arrhythmic cardiac SAE, and $0.8 \%$ non-cardiac SAE; $5.8 \%(95 \%$ CI $5.1 \%, 6.6 \%)$ patients in the conventional-cTn group suffered 30day SAE compared to $6.3 \%$ (95\% CI $5.0 \%, 7.9 \%$ ) in the hs-cTn group $(\mathrm{p}=0.54)$. The $\mathrm{LR}+, \mathrm{LR}-$ and unadjusted OR for 30-day SAE were: conventional cTn 6.4 (95\% CI 4.7, 8.8), 0.8 (95\% CI 0.8, 0.9) and 8.0 (95\% CI 5.5, 11.7); and hs troponin 2.5 (95\% CI 2.0, 3.2), 0.5 (95\% CI $0.4,0.7)$, and 5.0 (95\% CI 3.0, 8.4), respectively. The adjusted ORs were conventional-cTn 4.2 (95\% CI $2.7,6.5)$ and hs-cTn $2.1(95 \%$ CI $1.2,3.8)$. The AUC for the CSRS models with two assays were: conventional-cTn 0.87 (95\% CI 0.85, 0.89) and hs-cTn 0.88 (95\% CI $0.85,0.92)$.

Conclusion: We found no differences between the prognostic abilities of conventional and hs cTn assays when combined with the clinical and ECG CSRS items to predict 30-day SAE after ED disposition among patients with syncope. Our study suggests that physicians can use either, conventional or hs cTn assay, for riskstratification of ED syncope.

Keywords: Conventional troponin, High-sensitivity troponin, Syncope

\section{LO56}

External validation of a low HEAR score to identify emergency department chest pain patients at very low risk of major adverse cardiac events without troponin testing

\section{O'Rielly, MSc, J. Andruchow, MD, MSc, A. McRae, MD, PhD}

University of Calgary, Calgary, AB

Introduction: Recent studies have explored the utility of clinical characteristics to identify patients at very low risk of major cardiac events (MACE) without utilizing troponin testing. Specifically, the History, ECG, Age, Risk Factor (HEAR) score has been proposed to identify patients at sufficiently low risk of acute coronary syndrome that they may not require troponin testing. The objective of this study was to externally validate a very low HEAR score to identify emergency department (ED) patients with chest pain at very low risk of 30day MACE.

Methods: This was a secondary analysis of a completed prospective cohort study of patients requiring troponin testing to rule out myocardial infarction (MI) in a large urban ED. Exclusion criteria included clear ischemic changes or ST-elevation MI on ECG. We conducted two pre-planned analyses in specific cohorts: (1) patients with no documented history of coronary disease; and (2) all patients. In each patient cohort, the HEAR score was calculated for all patients, with HEAR $=0$ and HEAR $\leq 1$ tested as 'very low risk' thresholds. Sensitivity, specificity, and positive and negative predictive values at both cut-offs were quantified for the co-primary outcomes of 30-day MACE and index MI. All outcomes were two-physician adjudicated.
Results: Of 1150 total patients, 820 had no prior history of CAD. Of the 820 patients included in the primary analysis, $57(7.0 \%)$ and 64 (7.8\%) experienced index MI and 30-day MACE, respectively. In patients with no prior history of CAD, HEAR $\leq 1$ identified 202 (24.6\%) patients as very low risk with sensitivities of $98.3 \%(95 \% \mathrm{CI}$ 90.6-99.9) for index MI and 98.4\% (95\% CI 91.6-99.9\%) for 30-day MACE. Among all patients, HEAR $\leq 1$ identified $204(17.7 \%)$ patients as very low risk with sensitivities of $98.9 \%$ (95\% CI $94.3-$ 99.9) and $99.2 \%$ (95\% CI 95.6-99.9) for index MI and 30-day MACE, respectively.

Conclusion: A HEAR score $\leq 1$ can identify nearly $25 \%$ of ED chest pain patients without known CAD and up to $17 \%$ of all patients as very low risk for 30-day MACE and unlikely to benefit from troponin testing. Broad implementation of this strategy could lead to significant resource savings.

Keywords: Chest pain, Risk stratification

\section{LO57}

Can ED chest pain patients with intermediate HEART scores be managed as outpatients?

A. Moustapha, BA, A. Mah, BSc, A. Leach, MD, L. Roberts, BSc, MD, MSc, G. Kaban, MD, R. Zimmermann, MD, J. Orvold, MD, J. Shavadia, MD, L. Martin, MD, MN

University of Saskatchewan, Saskatoon, SK

Introduction: The HEART score is a well-validated risk stratification tool for chest pain that recommends patients with intermediate-risk scores be admitted for inpatient investigation and treatment, which is a resource-intensive process. This study aims to determine whether intermediate HEART score patients can be managed on an outpatient basis at rapid access chest pain clinics (RACPC) with a low incidence of major adverse cardiac events (MACE) by 6 weeks.

Methods: This retrospective observational study included all ED patients referred to outpatient RACPCs from January 2018 to April 2020 in Regina and Saskatoon, Saskatchewan. The primary outcome was the rate of MACE, a composite measure of death, acute coronary syndrome (ACS), stroke, coronary angiography, and revascularization at 6 weeks. Secondary outcomes were type of MACE and rate of MACE before RACPC appointment. Congruence between reviewers, ED physicians and cardiologists' assignment of HEART scores was assessed by kappa coefficient. Odds ratios (OR) were calculated from univariate and multivariate analysis of HEART score components to determine which were most predictive of 6-week MACE. Statistical significance was defined as a $95 \%$ confidence interval (CI) and a critical p-value of 0.05 .

Results: We found 2018 charts, of which 1989 were included as independent patient visits to the ED resulting in a RACPC referral. $5.3 \%$ of all patients and $9.3 \%$ of intermediate risk patients had a MACE at 6 weeks and no deaths occurred. Of all the MACE in the intermediate risk group, $2.6 \%$ occurred before their RACPC appointment. Coronary angiography was the most common MACE. Excluding coronary angiography, there was a $1.5 \%$ risk of MACE in intermediate-risk patients by 6 weeks. Components of the HEART score most predictive of MACE were troponin (OR 10.9, 95\% CI $4.3-$ $27.8)$ and history $(8.5,95 \%$ CI 4.6-15.6). Concordance between ED physicians and cardiologists was fair (0.34) and was good (0.54) between ED physicians and reviewers.

Conclusion: Outpatient RACPC management of patients with intermediate-risk HEART scores appears safe, is associated with a low risk of MACE at 6 weeks and could reduce costly inpatient admissions for chest pain.

Keywords: Chest pain, MACE, Outpatient 


\section{LO58}

Flattening the other curve: reducing emergency department delays for ST elevation myocardial infarction during the coronavirus disease 2019 pandemic

\section{J. McLaren, MD, L. Chartier, MD CM, MPH}

University of Toronto/University Health Network, Toronto, ON

Introduction: The coronavirus disease 2019 (COVID-19) pandemic has been associated with delays to door-to-balloon time for patients with ST-Elevation Myocardial Infarction (STEMI), despite reduced volumes of emergency department (ED) patients. However, little is known about ED providers' contribution to these delays. We sought to measure STEMI delays in the ED over the first 9 months of the pandemic.

Methods: This study was a multi-centre, retrospective chart review from two urban, academic medical centres. We obtained the number of ED visits and COVID-19 swabs per month from the hospital database, and the list of all ED Code STEMI patients with culprit lesion from the cardiac cath lab. We measured door-to-ECG time (DTE) from the triage time to the first ED ECG, and ECG-to-Activation time (ETA) from the first ED ECG to the time the cath lab was activated. We calculated median DTE and ETA times in 3-month intervals in 2020 corresponding to phases of the pandemic (pre-wave1, wave-1, and post-wave-1) and compared them to the 2019 baseline using Wilcox rank-sum test.

Results: In 2019 there were 9,931 ED visits/month, median DTE was 10.0 min and median ETA was 8.5 min. In January-March 2020, ED visits were $99.5 \%$ of baseline, there were 461 COVID-19 swabs/month, and there was no change in median DTE time $(\mathrm{p}=0.08)$ or median ETA time $(p=0.23)$. In April-June, there were 1688 COVID-19 swabs/month and ED visits fell to $60.1 \%$ of baseline, during which time there was a significant increase in median DTE time (10.0-33.5 min, $\mathrm{p}<0.01)$ and median ETA time $(8.5-25.0 \mathrm{~min}$, $\mathrm{p}<0.01$ ). In July-September, there were 1420 COVID-19 swabs/month and ED visits increased back to $81.3 \%$ of baseline, during which time metrics returned approximately to baseline: median DTE $8 \min (\mathrm{p}=0.29)$ and median ETA $11 \min (\mathrm{p}=0.28)$.

Conclusion: During the first wave of the pandemic, ED volumes fell $40 \%$ and there was an approximate tripling in both DTE and ETA times, representing delays in triage and diagnosis of STEMI patients, respectively. In the subsequent 3 months, despite a partial return of normal ED volumes and ongoing COVID-19 activity demonstrated by high number of COVID-19 swabs, times normalized. This shows that ED providers adapted to their new environment to ensure high-quality care for patients, and the reasons why will be crucial to understand as we move through subsequent waves of the pandemic.

Keywords: Coronavirus disease 2019, ST Elevation Myocardial Infarction

\section{LO59}

Epidemiology, mortality and miss rate of acute aortic syndrome in Ontario, Canada: a population-based study

R. Ohle, BA, MBChB, MSc, D. Savage, MD, PhD, S. McIsaac, MBChB, MEd, J. Caswell, PhD, K. Yadav, MD, MSc, M. Conlon, $\mathrm{PhD}$

\section{Health Science North, Sudbury, ON}

Introduction: Acute aortic syndrome is a life-threatening aortic emergency. It describes three distinct diagnosis: acute aortic dissection, acute intramural hematoma and penetrating atherosclerotic ulcer. There are currently no accurate Canadian estimates for incidence, mortality or misdiagnosis. The incidence of the disease is integral to resource allocation for cost-efficient care. The mortality and misdiagnosis are important in order to identify areas for improvement of care. Our objectives were to determine incidence, mortality, and miss rate of acute aortic syndrome.

Methods: A population-based retrospective cohort study of anonymously linked data for residents of Ontario, Canada, was carried out. Incident cases of acute aortic syndrome were identified between 2013 and 2018 using a validated algorithm based on ICD codes, length of stay and death. We explored Incidence (number of cases/population of Ontario), mortality, and miss rate (defined as seen in the emergency department in preceding 14 days to AAS diagnosis with a presenting complaint consistent with AAS).

Results: There were 2808 cases of acute aortic syndrome [mean age (SD) $68.03 \pm 14.70$, male $1744(62.11 \%)$, rural residence 357 $(12.71 \%)]$ over the study period. Overall incidence for acute aortic syndrome was 3 per 100,000 and 1194 (42.45\%) died. In the 14 days prior to diagnosis $22.5 \%$ of patients were seen in the emergency department with a complaints consistent with AAS.

Conclusion: Incidence of AAS is similar to other population-based studies. Mortality is twice that of other developed countries (United States, Germany and Japan). The miss rate for AAS could be as high as $22.5 \%$ of cases. Our study demonstrates the need for improved patient care around the diagnosis and treatment of this deadly condition.

Keywords: Acute aortic syndrome, Misdiagnosis

\section{LO60}

The clinical impact of a new pathway to guide anticoagulation prescription for atrial fibrillation in the emergency department: a 2-year evaluation

J. Carter, BSc, C. Kirwan, BSc, MD, S. Niaz, MD, S. Baweja, BSc, F. Al-Haimus, MBChB, Y. Hu, N. Clayton, K. de Wit, BSc, MBChB, $\mathrm{MD}, \mathrm{MSc}$

\section{McMaster University, Hamilton, ON}

Introduction: The Canadian Cardiovascular Guidelines advise that emergency physicians initiate oral anticoagulation (OAC) therapy for patients who have atrial fibrillation (AF) and are CHADS65 positive. Our previous chart review showed only $17 \%$ of eligible patients were being started on OAC in our two emergency departments (EDs). We developed and implemented a pathway for initiation of OAC in AF. Our aim was to determine the rate of pathway use and to compare the 90-day incidence of stroke and major bleeding among patients eligible for $\mathrm{OAC}$ prescription in both groups (those treated with and without the pathway).

Methods: We performed a chart review between 1st June 2018 and 31st May 2020, of patients from two Hamilton EDs with a discharge diagnosis of $\mathrm{AF}$ (or flutter) who were discharged home. Inclusion criteria were age 18 or older, with ECG confirmed AF (new or previously known). The pathway was a hard copy form which documented contra-indications to OAC, the elements of the CHADS65 score, and the anticoagulant prescription. The pathway was scanned into the patient's medical record after discharge. Trained researchers abstracted data on the exposure of interest by the presence or absence of the pathway in the electronic medical chart. Patients were followed forward in time for 90 days by electronic medical record review, to document subsequent diagnoses of stroke, transient ischemic attack, arterial embolism and major bleeding events. All events were independently adjudicated.

Results: In total, 766 patients with $\mathrm{AF}$ who were discharged from the ED were included, of whom 264 were already taking OAC, 166 were CHADS65 negative and 65 had a contraindication to OAC, leaving 
271 patients eligible for OAC prescription. Among the 271 eligible for OAC, 166 were treated with the pathway: $137(83 \%)$ were initiated on OAC, one of whom had a major gastrointestinal bleed and one patient who decline OAC had a stroke. Among patients eligible for OAC, 105 were treated without the pathway: 24 (23\%) started OAC, one of whom had a major intramuscular bleed. There was no stroke/ arterial embolism. There were no statistically significant differences in 90-day stroke or major bleeding rates between patients treated with or without the pathway.

Conclusion: Use of our new pathway increased the prescription of $\mathrm{OAC}$ in eligible AF patients. This did not translate into a difference in the 90-day stroke or bleed rate.

Keywords: Anticoagulation, Atrial fibrillation, Stroke

\section{LO61}

\section{Assessing the learning impact of the Northern City of Heroes public cardiopulmonary resuscitation exhibit at Science North in Sudbury, Ontario}

M. Moskalyk, BSc, R. Ohle, MBChB, MSc, MA, A. Henson, BSc, M.S.Com, K. Pisani, BA, MSc, S. McIsaac, MBChB, MEd

Department of Undergraduate Medicine, Queen's University, Kingston, Ontario, Canada, Kingston, ON

Introduction: Survival from out-of-hospital cardiac arrest (OHCA) in Canada is less than $10 \%$. The Northern City of Heroes $(\mathrm{NCH})$ initiative was launched in Sudbury, ON with a goal of improving OHCA survival by improving access to compression-only CPR (CO-CPR) training. NCH launched the first interactive CO-CPR exhibit at Science North, a science centre in Sudbury. The goal of this study was to assess the impact of the exhibit on learning CO-CPR.

Methods: We performed a cross-sectional study of self-selected visitors to assess the exhibit learning impact using self-reported surveys and video-taped coded visitor behaviours. Using surveys, we assessed the change in proportion of those willing to perform CPR pre and post exhibit interaction and analyzed behaviours using the Visitor Engagement Framework (VEF) to measure Initiation, Transition, and Breakthrough behaviours. The VEF is a validated tool used to measure the impact of exhibits. Breakthrough is the deepest form of engagement that reflects high level connections made through prior knowledge and further inquiry. A highly impactful exhibit is defined as breakthrough achieved in $>25 \%$ of visitors. Concurrent triangulation strategy, collecting qualitative and quantitative data simultaneously, was used to cross-validate and corroborate the findings. A sample size of 384 was calculated based on a $95 \%$ confidence interval with a margin of error $\pm 5 \%$ for breakthrough behaviour assessment.

Results: 398 visitor interactions were coded using the VEF. 208 $(52.2 \%)$ visitors were female, majority of coded visitors were adults (20-64 years) $(39.7 \%)$. Initiation behaviour was achieved in $53.5 \%$ (95\% CI 48.9-58.1), Transition behaviour in $15.8 \%$ (95\% CI $7.53-$ 24.1) and Breakthrough behaviour in 30.6\% (95\% CI 23.8-37.4). 424 pre-exhibit and 118 post-exhibit surveys were completed. There was a $7.4 \%$ (95\% CI $5.3-9.9 \%$ ) increase in the likelihood of performing CPR on a friend or family [pre-exhibit $\mathrm{n}=238(56.2 \%)$, post-exhibit $\mathrm{n}=75(63.6 \%)]$.

Conclusion: The $\mathrm{NCH}$ exhibit was deemed a highly impactful learning environment. This novel form of asynchronous CPR education has the potential to remove barriers of traditional CPR training, reach broad audiences and have a measurable impact on bystander CPR and OHCA survival. Limitations may be found in the focused demographics as well as the nature of self-reported learning. Future research directions include broader surveying to assess improvements in community response to OHCA as a direct result of interactive bystander CPR exhibitry.

Keywords: Bystander CPR, Informal learning, Innovations in EM education

\section{LO62}

Take a breath: investigating the effect of box-breathing on the stress, cognitive load, and performance of junior residents in a simulation-based resuscitation OSCE

S. Van Heer, BSc, G. Gutiérrez, BSc, A. Szulewski, MD, MHPE, PhD, T. Chaplin, MD

Queen's University, Kingston, ON

Introduction: Resuscitating an unwell patient can be an overwhelming experience for junior residents. Cognitive load theory suggests the inherent complexity and stress of resuscitations (intrinsic cognitive load-ICL) and off-task distractions (extraneous cognitive load-ECL) can overload cognitive capacity and impair performance. Box Breathing (BB) can mitigate this stress response and maintain performance despite a stressful environment. We aimed to investigate the effect of $\mathrm{BB}$ on residents' stress, cognitive load, and performance. Methods: Thirty-nine $(\mathrm{n}=39)$ "Foundations of Discipline" (PGY1) level residents from 14 specialties participated in a summative simulation-based resuscitation OSCE. These residents were randomized to perform either $\mathrm{BB}$ or a control colour naming $(\mathrm{CN})$ task for $20 \mathrm{~s}$ prior to each of the four OSCE stations. Residents completed the STAI-6 and a modified Leppink cognitive load questionnaire after each station. Faculty assessors observed each performance and assigned an entrustment (EPA) score. Intervention arms were compared with unpaired student's t test, and linear regression models examined relationships between stress, cognitive load, and entrustment scores.

Results: Compared to the $\mathrm{CN}$ group, the $\mathrm{BB}$ group reported nonsignificantly lower stress $(13.8$ vs $14.5, p=0.1452)$, lower total cognitive load (6.47 vs 6.99, $\mathrm{p}=0.249)$, and higher EPA scores $(4.12$ vs $4.01, p=0.4028)$. BB group reported significantly lower ICL than the $\mathrm{CN}$ group (4.03 vs $4.86, \mathrm{p}=0.0011$ ). Also, BB had a significant effect $(p=0.019)$ on the relation between the ICL and the EPA scores, such that higher ICL amongst the $\mathrm{CN}$ group was associated with decreased EPA scores $(b=-0.149, p=0.0177)$, but the BB group's EPA scores were not associated with ICL. Amongst all participants, STAI-6 scores were significantly associated with higher total cognitive load $(b=0.277, p=0.0003)$, both ICL $(b=0.164$, $\mathrm{p}=0.0002)$ and ECL $(\mathrm{b}=0.113, \mathrm{p}=0.0452)$, and lower EPA scores $(\mathrm{b}=-0.074, \mathrm{p}=0.0005)$.

Conclusion: Residents using BB had significantly lower ICL and increased tolerance for ICL without affecting EPA scores. Suggesting that the psychophysiological changes promoted by BB are protective against cognitive overload by excessive ICL, which would otherwise affect performance. Additionally, we showed that higher levels of stress can lead to significantly higher levels of cognitive load and lower EPAs. Future work will explore the impact of BB on learning and performance in resuscitation.

Keywords: Cognitive load, Residents, Resuscitation

\section{LO63}

Do preparatory online modules optimize cognitive load during simulated resuscitation scenarios?

G. Gutiérrez, BSc, S. Van Heer, BSc, T. Chaplin, MD 
Queen's University, Kingston, ON

Introduction: Simulation can provide a safe and reproducible learning experience; however, this environment can trigger high levels of cognitive load (CL) in learners. CL comprises the complexity of the task (intrinsic CL-ICL), off-task stimuli (extraneous $\mathrm{CL}-\mathrm{ECL}$ ) and forming schemas to learn from the task (germane $\mathrm{CL}-\mathrm{GCL}$ ). $\mathrm{CL}$ is a limited resource and, if overwhelmed, can negatively impact learning and performance. Thus, in this project we aimed to optimize CL by providing preparatory online modules to the learners before simulation sessions, to improve performance and guide simulation curricular development.

Methods: The Nightmares course is a simulation-based curriculum intended to teach and assess resuscitation skills. Fifty-three residents in their first postgraduate (PGY-1) year of training from 11 different programs participated in the course and were randomly assigned to either the modules group (MG) or control group (CG). Prior to each of the four simulation sessions, the MG received an online preparatory module (5-10 min-long); the CG did not receive any modules. Each simulation session consisted of 3 resuscitation scenarios. After each scenario, the resident participating as the leader and two residents who observed the scenario completed the Leppink cognitive load questionnaire. The leader's performance was assessed by an attending physician using an entrustment score (EPA).

Results: The preparatory online modules had a significant effect ( $p=0.044)$ on the ECL of the scenario leaders, with the CG having an increasing trend over four sessions $(b=0.33, p=0.0016)$ and the MG having a decreasing trend over four sessions $(b=-0.16$, $p<0.0001)$. We also found a significant change $(p=0.0027)$ in the relation of the ICL vs EPA of the MG and CG. With the CG having a decreasing trend in the relation between ICL and EPA $(b=-0.10$, $\mathrm{p}<0.0001)$ and the MG having an increasing trend in this relation $(\mathrm{b}=0.041, \mathrm{p}<0.0001)$.

Conclusion: In the context of PGY-1 residents participating in a simulation-based resuscitation curriculum, the use of preparatory online modules resulted in a significant lowering trend in the ECL over four sessions and a significant increasing trend in the relation between ICL and EPA. Since CL is a limited resource, a lower ECL allows more space for ICL to be used effectively to complete the task without resulting in cognitive overload. Thus, suggesting that the modules had an optimizing effect on the cognitive load of the residents.

Keywords: Cognitive load, Online modules, Simulation training

\section{LO64}

The influence of observation type (direct or indirect) on quality of workplace-based assessment written comments

J. Landreville, MD, MEd, J. Frank, MD, MEd, T. Wood, PhD, W. Cheung, MD, MEd

University of Ottawa, Ottawa, ON

Introduction: Over the last 20 years, there has been a global movement within postgraduate medical education towards competency-based medical education (CBME). The implementation of CBME has led to widespread changes across training programs within Canada including the discipline of Emergency Medicine. An emerging tension within CBME relates to the challenges and barriers associated with direct observation. Given this tension, the role of indirect observation within workplace-based assessment (WBA) has become of increased interest to medical educators. The aim of this research study was to determine the influence of observation type (direct or indirect) on quality of WBA documentation within a CBME curriculum.
Methods: To accomplish this aim, a retrospective quantitative analysis of short, workplace-based comments contained within entrustable professional activity (EPA) assessments was performed. EPA assessment data was collected and categorized into two groups based on what type of observation occurred (direct or indirect) $(n=244)$. Four physicians blinded to the type of observation, trainee and supervisor rated the quality of each EPA assessment using a previously published three-item instrument known as the Quality of Assessment for Learning (Qual) score. Using factorial analysis of variance, a comparison of mean Qual scores between the direct and indirect observation groups was conducted. The reliability of the Qual for EPA assessments was determined using a generalizability analysis A decision study was then performed to determine how many physician raters are required to produce reliable ratings.

Results: The type of observation (direct or indirect) did not contribute to any significant difference in quality between EPA assessments after being rated by physician raters using the Qual score $(\mathrm{F} 1210=1.89$; $P=0.17)$. The majority of variance in Qual score $(44 \%)$ was attributable to the EPA assessment itself. Three physician raters are required to produce reliability of 0.79 whereas four physician raters produced a reliability of 0.84 .

Conclusion: To the author's knowledge, this study is the first of its kind to determine the influence of observation type (direct or indirect) on quality of WBA documentation. Given that observation type did not influence the quality of WBA documentation, this study raises further questions regarding how direct and indirect observation truly differ and the implications for competence committees responsible for making judgements related to trainee promotion.

Keywords: Observation, Workplace-based assessment

\section{LO65}

Is a homemade cardiopulmonary resuscitation (CPR) trainer non-inferior to a commercial CPR trainer in teaching high quality compression-only CPR?

M. Moskalyk, BSc, R. Ohle, MBChB, MSc, MA, E. Boissonneault, BSc, A. Bilgasem, E. Tissot, MBChB, MEd

Department of Undergraduate Medicine, Queen's University, Kingston, Ontario, Canada, Kingston, ON

Introduction: Hands-on CPR education with a feedback mannequin is essential for skill acquisition. However due to the COVID-19 pandemic, there has been a halt to all in-person training. To mitigate these challenges, the Northern City of Heroes CPR student-led team developed an online interactive CPR training session incorporating a novel homemade CPR feedback trainer, which consists of a pickle jar lid with two toilet paper rolls placed on top. Our main objective was to assess if learning CPR on a homemade toilet paper (TP) trainer was non-inferior to a commercially available mannequin $(\mathrm{CM})$ when comparing post-training CPR scores

Methods: We conducted a non-inferiority randomized control trial of visitors attending Science North, a science centre in Sudbury, ON. Participants were randomized using opaque sealed envelopes and variable block randomization with block sizes 4,6 or 8 to a 10-min training session on either a TP or CM trainer. Overall CPR score was assessed on a separate feedback enabled mannequin. Primary outcome was mean overall CPR score as determined by the high fidelity mannequin software, which uses a combined weighted score for depth, rate, full release, hand position, no flow time and compression number per cycle. Secondary outcomes included proportion of participants who achieved a passing $>70 \%$ score and the rate, recoil and depth achieved. A sample size of 39 per group was calculated based on a $90 \%$ power to assess for the lower limit of a two-sided $95 \%$ 
confidence interval above the non-inferiority limit of $10 \%$, and a standard deviation of total CPR score of $15 \%$.

Results: 125 participants were randomized to the TP $(n=64)$ or CM trainer $(n=61)$. There was no significant difference between groups in age $(\mathrm{p}<0.06)$, sex $(\mathrm{p}<0.23)$, height $(\mathrm{p}<0.64)$, weight $(\mathrm{p}<0.69)$ or previous CPR training $(\mathrm{p}<0.43)$. There was an absolute difference of $2 \%$ (CI 95\% -3.3 to 7.3\%) in mean overall CPR score between groups [TP $=82 \%$ (SD 15.9\%), $\mathrm{CM}=84 \%$ (SD $15 \%$ )]. There was an absolute $4 \%$ (CI $95 \%-17.1$ to $9 \%$ ) difference in passing CPR score $(>70 \%)$ between groups $[\mathrm{TP}=52(81.2 \%)$ vs. $\mathrm{CM}=52(85.2 \%)]$. Adequate rate (100-120 compressions $/ \mathrm{min})$ was achieved in $\mathrm{TP}=56(87.5 \%)$ vs. $\mathrm{CM}=54(88.5 \%)(\mathrm{CM}), 5 \mathrm{~cm}$ depth in $\mathrm{TP}=61(95.3 \%)$ vs. $\mathrm{CM}=52(82.5 \%)$ and recoil in $\mathrm{TP}=38$ $(59.4 \%)$ vs. $\mathrm{CM}=44(72.1 \%)$.

Conclusion: A homemade CPR trainer was non-inferior to a commercially available trainer. This study provides preliminary evidence supporting the use of a homemade, easily accessible trainer for basic compression-only CPR skill acquisition.

Keywords: compression-only CPR, homemade CPR trainer, remote learning

\section{LO66}

\section{The catalytic effect of multisource feedback for trauma team} learners: a pilot study

\section{Allen, MSc, BASc, T. Chaplin, MD}

Queen's University, Kingston, ON

Introduction: Providing feedback and assessment to learners in the context of a dynamic trauma resuscitation is challenging. Multisource feedback (MSF) is a workplace-based assessment method whereby each interacting team member provides feedback to a specific individual. Despite the growing use of MSF in team-based medical specialties, it has not been studied in trauma teams. This work aims to measure MSF's impact on the practice change (catalytic effect) of trauma team captains (TTCs).

Methods: This study took place at a level one trauma centre between July 2019 and January 2020. TTCs were senior postgraduate medical residents and either received MSF from members of the trauma team or standard feedback following trauma cases. Following the reception of feedback, the impact on practice change was captured via questionnaires distributed immediately (Q1) and 3-week later (Q2). Comments were collected related to the perception of MSF in a trauma context.

Results: Data collection was stopped after 24 TTCs participated as data saturation was achieved. Twelve received MSF, and 12 received standard feedback. In the standard feedback group, only seven $(60 \%)$ of the TTCs received feedback, which was always delivered verbally by the faculty trauma team leaders. All 12 TTCs in the MSF group received feedback. There was no significant difference in the catalytic effect of MSF compared to standard feedback. Q1 found that both groups had positively influenced intention for practice change (4.1 on a 5-point Likert scale for the MSF group vs. 4.4 for the standard feedback group, $\mathrm{p}=0.051$ ). Three weeks later, $\mathrm{Q} 2$ found a reduced intention for practice change in both groups ( 3.5 in the MSF group vs. 3.4 in the standard feedback group, $p=0.643$ ). Trauma team members reported overall favourability about the MSF process (Likert scores ranged from 3.5 to 4.6). Four major themes were identified via narrative comments by team members who participated in the MSF process: "logistics", "usefulness", "applicability", and "team". Although "helpful" was a comment frequently stated, analyses also identified "efficiency" and "feasibility" as MSF's main challenges in trauma.

Conclusion: This pilot study found that MSF does not result in a selfreported catalytic effect for TTCs when compared to standard feedback. The incorporation of MSF into a trauma-team context is possible, but feasibility and efficiency were identified as significant challenges.

Keywords: Catalytic effect, Multisource feedback, Trauma teams

\section{LO67}

Building validity evidence for the QuAL (Quality of Assessment for Learning) score as a measure of the quality of narrative comments in Competency Based Medical Education

R. Woods, MD, MMed, S. Singh, B. Thoma, MD, MSc, MA, C. Patocka, MD, MHPE, W. Cheung, MD, MMed, S. Monteiro, PhD, T. Chan, MD, MHPE

University of Saskatchewan, Saskatoon, SK

Introduction: Providing useful feedback to medical trainees is a challenge. Competency based medical education (CBME) relies heavily on narrative comments from entrustable professional activities (EPA) for programmatic assessment. There is validity evidence supporting the QuAL (Quality of Assessment for Learning) score for rating narrative comments within workplace-based assessments, but its utility for rating EPAs has not been evaluated. We sought to establish validity evidence for the QuAL score in the context of CBME by studying the perspectives of residents, academic advisors and competence committee members.

Methods: 52 de-identified narrative comments were selected from two emergency medicine EPA databases using purposeful sampling. Six collaborators (two residents, two academic advisors, and two competence committee members) were recruited from each of four EM Residency Programs (Saskatchewan, McMaster, Ottawa, and Calgary). The collaborators were divided into four rater cohorts to rate a sample of these comments with a utility score and the QuAL score. Correlation between utility and the QuAL score were calculated using Pearson's correlation coefficient. Sources of variance were calculated using a generalizability study and reliability was assessed using inter-rater reliability.

Results: All 24 invited collaborators completed the full study. The QuAL score and utility score had a high positive correlation amongst the residents $(\mathrm{R}=0.8)$ and academic advisors $(\mathrm{R}=0.75)$ and a moderately high correlation amongst competence committee members $(\mathrm{R}=0.68)$. The absolute g-coefficient ranged from 0.79 to 0.91 , indicating the major source of variance was the narrative comment, with minimal variance from the raters. The inter-rater reliability ranged from 0.74 to 0.94 among the cohorts of raters.

Conclusion: The QuAL score is a simple tool that provides a reliable measurement of the quality of narrative comments in EPA assessments. It correlates well with utility for the for three different end user groups in CBME: residents, academic advisors and competence committee members. It can serve as a tool for faculty development through providing a framework for writing narrative comments, or through creating report cards of faculty performance in audit and feedback programs. It can also be used as an outcome measure for program evaluation in CBME, indicating the percentage of comments in a program meeting a standard of quality.

Keywords: Assessment, Faculty development, Program evaluation

\section{LO68}

Point-of-care ultrasound in pediatric advanced life support: perspectives of new graduates on the utility of point-of-care ultrasound in pediatric resuscitation

G. Bravo, MD, T. Lynch, MD, M. Miller, PhD, K. Van Arsen, MSc Children's Hospital, London, ON 
Introduction: Point-of-care ultrasound (POCUS) has been incorporated into Advanced Care Life Support (ACLS) guidelines to determine the presence of potentially reversible causes of cardiac arrest that would merit continued resuscitative efforts (e.g. pneumothorax, cardiac tamponade). The pediatric counterpart, Pediatric Advanced Life Support (PALS), currently has no formal POCUS applications. As POCUS becomes a core competency for emergency medicine trainees we postulated that trainees may see it as standard of care not only in adults, but also in pediatric patients. The objective of this study was to determine from the perspective of senior emergency medicine residents and pediatric fellows what POCUS applications they would employ, if any, in standardized PALS scenarios.

Methods: With permission of the Heart and Stroke Foundation of Canada, a scenario-based Redcap survey using four PALS vignettes was e-mailed to final year emergency medicine trainees. Surveys were distributed via Program Directors and Chief Residents of the FRCPC, Pediatric Emergency Medicine Fellowship, and CCFP-EM Programs. English and French versions were distributed from Jan 2020 to Jun 2020 to all 41 Canadian programs. Trainees were asked to indicate their POCUS applications and technical confidence for each scenario and application. Participants were compensated with a \$5 Amazon gift card, which was funded by an internal grant.

Results: Trainees indicated POCUS application in all scenarios. The majority of trainees indicated use for volume status evaluation (79\%) and IV access (86\%) in hypovolemic shock; pneumothorax identification $(90 \%)$ and endotracheal tube depth evaluation $(56 \%)$ in an intubated patient with difficult ventilation; cardiac function evaluation (96\%) and volume status (57\%) in a myocarditis patient; and determination of cardiac standstill (94\%) in an arrest. Regarding confidence in overall POCUS skills, trainees identified themselves to be very confident $(9.6 \%)$, generally confident $(42 \%)$, somewhat confident $(46 \%)$ and not confident $(1.9 \%)$ with highest confidence in pneumothorax identification (55\% indicated very confident).

Conclusion: The majority of trainees indicated they would use POCUS in pediatric resuscitation, which suggests a need for further discussion of incorporation of POCUS into PALS guidelines. However, the confidence level in POCUS skills at the end of training is low, which warrants further investigation into teaching methods, curriculum development, and assessment of POCUS skills during training.

Keywords: PALS, Pediatric, Point-of-care ultrasound

\section{LO69}

Careful anticoagulation review in emergency medicine: the CARe-EM study

A. Tan, BSc, MD, F. Germini, MD, S. Farquharson, BSc, T. Rashid, MBChB, N. Clayton, M. Ali, MBBS, S. Ramsden, MD, Y. Hu, S. Niaz, MBChB, C. Kirwan, BSc, MD, K. de Wit, BSc, MBChB, $\mathrm{MD}, \mathrm{MSc}$

\section{Queen's University, Kingston, ON}

Introduction: A growing number of Canadians take anticoagulant medication, and many emergency patients are anticoagulant users. An emergency department (ED) visit could be an opportunity to reassess the bleeding risk among anticoagulant users, with a view to mitigating future bleeding. The aim of this study was to assess the performance of existing bleeding scores in anticoagulated ED patients.

Methods: This city-wide health records review included consecutive adult anticoagulant users presenting to two EDs for any complaint. We excluded patients living outside the hospital catchment area and those started on anticoagulation in the ED. Trained researchers abstracted exposure and outcome data from electronic health records following a standardized protocol with independent data validation.
The primary outcome was the incidence of major or clinically relevant bleeding as defined by International Society of Thrombosis and Hemostasis (ISTH) occurring after the index ED visit and within 12 months. The secondary outcomes were (1) major bleeding within 12 months, (2) major or clinically relevant bleeding within 3 months of the index ED visit. All bleeds were independently adjudicated. The performance of bleed scores (ATRIA, HAS-BLED, HEMORR2HAGES and VTE-BLEED) were assessed with the area (AUC) of the receiver operating characteristics (ROC) curves.

Results: The study included 521 patients, median age 78, 55\% male. 165 took apixaban, 163 warfarin, 121 rivaroxaban, 56 dabigatran, 16 heparin. Indication for anticoagulation was predominately atrial fibrillation $(64 \%)$, venous thrombosis $(16 \%)$ and prosthetic heart valve $(6 \%)$. Common co-morbidities were hypertension $78 \%$, diabetes $33 \%$, heart failure $31 \%$, stroke $26 \%$ and renal impairment $17 \%$. 38/521, $7.3 \%,(5.4 ; 9.8 \%)$ patients had a major $(n=14)$ or clinically relevant bleed $(\mathrm{n}=24)$ within 12 months. This included gastrointestinal 11 $(2 \%)$, intracranial $5(1 \%)$, hematuria $9(2 \%)$, epistaxis $8(2 \%)$ and other sites $5(1 \%)$. The ROC curve AUCs for major or clinically relevant bleeding within 12 months were ATRIA $0.62(0.53 ; 0.71)$, HAS-BLED $0.56(0.48 ; 0.65)$, HEMORR2HAGES $0.60(0.51 ; 0.70)$ and VTE-BLEED $0.57(0.47 ; 0.66)$. The $95 \%$ confidence intervals for the secondary outcome AUCs crossed 0.50 with the exception of ATRIA $0.68(0.55 ; 0.82)$ and HEMORR2HAGES $0.69(0.55 ; 0.83)$ for major bleeding at 12 months.

Conclusion: Existing bleeding scores did not predict or were poorly predictive of anticoagulant-associated bleeding in our ED cohort.

Keywords: Bleeding, Prediction scores

\section{LO70}

Diagnostic accuracy of non-invasive imaging modalities versus direct visualization to diagnose adult epiglottitis: a systematic review

E. Creasor, BSc, MD, K. Harper, BN, MD, R. Ohle, MBChB, MD

Northern Ontario School of Medicine, Sudbury, ON

Introduction: Epiglottitis can be a severe, life threatening illness which is challenging to diagnose. Direct visualization is the gold standard for diagnosis, however this requires specialized skills and equipment. To date, there is a lack of consensus as to which method of non-invasive imaging is best utilized to diagnose these patients. Our aim was to assess the accuracy of non-invasive imaging modalities including lateral neck radiographs, computed tomography (CT) and bedside ultrasound, to diagnose epiglottitis in adults presenting to the emergency department with symptoms concerning for epiglottitis.

Methods: We completed a librarian-assisted search of Pubmed, Medline, EMBASE and Cochrane Databases from 1980 to December 2020. A snowball sampling method was used to search the citations of included studies. No restrictions for language or study type were imposed. Two reviewers independently reviewed and extracted data from the relevant articles. Agreement measured by Kappa statistic. These included prospective and retrospective studies. The gold standard comparison was direct visualization (fiberoptic scope or laryngoscopy). Study quality was assessed using the QUADAS-2 tool. Both clinical and statistical heterogeneity precluded meta analysis of studies.

Results: 6 studies with 1843 patients were included. Lateral neck Xray results varied depending on which radiologic sign was utilized; epiglottic width $>6.3 \mathrm{~mm}$ had a sensitivity of $96.2 \%$ (95\% CI 93 97.9), specificity $98.2 \%(95 \%$ CI $97.3-98.8)(n=1638)$, thumb sign a sensitivity of $65.9 \%$ (95\% CI $55.3-75.5$ ) and specificity of $99.5 \%$ $(95 \%$ CI $96-100)(n=1836)$, vallecula sign a sensitivity of $53.8 \%$ 
(95\% CI 43.6-63.7) and specificity of $99.5 \%$ (95\% CI 91.9-100) $(\mathrm{n}=1836)$ and aryepiglottic fold width $>8.7 \mathrm{~mm}$ had sensitivity of $86.5 \%$ (95\% CI 81.8-90.2) and specificity $78.7 \%$ (95\% CI $76.3-81$ ) $(\mathrm{n}=1638,95 \%)$. CT and bedside ultrasound techniques to diagnose epiglottitis have not been well studied, no data met inclusion criteria. Conclusion: At this time, there is weak evidence to support the use of one non-invasive diagnostic modality over the other. The gold-standard for diagnosing epiglottitis remains direct visualization, however lateral neck X-rays assessing epiglottic width demonstrate promise for an easier, non-invasive method for diagnosis.

Keywords: Epiglottitis, Supraglottitis

\section{LO71}

\section{Utilization of CT angiography of the head and neck in the era} of endovascular therapy: a retrospective study

L. Salehi, BSc, MD, MPH, J. Jaskolka, MD, M. Ossip, MD, P. Phalpher, MD, R. Valani, MBA, MD, M. Mercuri, MSc, PhD

William Osler Health System, Brampton, ON

Introduction: In 2015, several landmark trials showed clear benefits of endovascular therapy (EVT) in select patients presenting with an acute ischemic stroke with large vessel occlusion (LVO). There has since been a scale-up and expansion of EVT availability within Ontario and Canada. However, only a small fraction of those patients suspected of an acute ischemic stroke will be candidates for EVT. In August 2016, our site introduced a code stroke protocol which integrates CT angiography (CTA) in addition to a non-contrast head $\mathrm{CT}$ in the evaluation of patients with a suspected acute ischemic stroke within the time window for EVT. Patients who are potential candidates for EVT are transferred to the closest referral centre with EVT capabilities. The objective of this study is to assess the impact of this shift in best practice guidelines on (1) the volume of CTAs ordered in the ED, and (2) the number of actionable findings on these CTAs, defined as those that lead to a transfer to a referral centre.

Methods: Clinical and administrative data was collected on all head and neck CTAs ordered within the ED at two high-volume community hospitals and an affiliated urgent care centre during the 6-year period between January 1, 2014 and December 31, 2019. Of those patients who received CTAs, we identified those who were then transferred to a regional referral centre, and, specifically, to a regional stroke centre for potential therapy with EVT.

Results: A total of 4719 CTAs were ordered during the 6-year period. There was nearly a 16 -fold rise in the number of CTAs ordered per year, from 108 (in 2014) to 1648 (in 2019). A total of 164 patients who underwent CTAs $(3.48 \%)$ were ultimately transferred to a regional tertiary care centre, of which $43(0.91 \%)$ were transferred to a regional stroke centre for consideration of EVT. Of these, 20 received EVT, and 11 were deemed not candidates for EVT. Data is not available for the remaining 12 patients. Of the 20 patients who underwent EVT, six died in-hospital, five were discharged to Long-Term Care or Complex Continuing Care facilities, and nine were discharged home or to a rehabilitation facility.

Conclusion: Little is known of the impacts on healthcare resources that have resulted from the system-wide changes made necessary by the widespread adoption of EVT. Our study shows that, within our site, these system changes have resulted in large increases in CTA utilization with very small numbers of patients ultimately undergoing successful EVT.

Keywords: Computed tomography angiography, Neuroimaging, Stroke

\section{LO72}

\section{The effect of point of care ultrasound on helicopter EMS scene times}

K. Onotera, S. Ryan, T. Jelic, MD

University of Manitoba, Winnipeg, MB

Introduction: Point of care ultrasound (PoCUS), specifically the extended focused assessment with sonography in trauma (E-FAST), has become an integral component of surveying trauma patients in the acute care environment. In Canada, many of our rural trauma patients are transported to trauma centres by helicopter emergency medical services (HEMS). The HEMS environment is unique as the noise and vibration can render physical exam skills useless. PoCUS lends itself as an ideal tool for this domain, providing real-time information to evaluate patient conditions and guide management. PoCUS use in HEMS can result in expedited time to in-hospital interventions. However, there is limited evaluation of what impact performing fieldbased ultrasound has on the amount of time at the scene and if this delays transport. This study aims to assess the impact of the implementation of a HEMS ultrasound program on scene times.

Methods: This is a retrospective review of all missions performed at the Shock Trauma Air Rescue Service (STARS) Manitoba base. Air medical crew (AMC) logged their image interpretation in the patient record and stored all images. These images, interpretations and clinical integration were reviewed and compared to a gold standard (either CT imaging or operative report). All QA was performed by a physician with a fellowship in emergency ultrasound. Scene time information was collected from the patient record for each mission. Scene time was defined from when AMC arrived at the patient to when patient packaging for departure was completed. Secondary outcomes were the accuracy of point of care ultrasound interpretation by AMC compared to a gold standard.

Results: In total, 231 patients were transported with an ultrasound performed, and 1016 were transported without. Mean scene time in the PoCUS group was $25.98 \mathrm{~min}( \pm 3.1895 \% \mathrm{CI})$ compared to $26.99 \mathrm{~min}( \pm 1.4495 \% \mathrm{CI})$ in the non-ultrasound group with no statistical difference $(\mathrm{p}<0.1336) .174$ scans were performed in trauma patients, the remaining were medical. 61 patients were scanned during flight, 79 in field, and 91 in hospital. AMC had a pooled sensitivity of 53.6 and specificity of 98.9 . False-positive/negative scans had no impact on patient care or outcomes.

Conclusion: This study demonstrates that introducing a PoCUS program into HEMS did not result in a delay in scene times. AMC are able to perform appropriate image acquisition and interpretation to a high level of accuracy, diagnostic certainty and appropriate clinical integration.

Keywords: Helicopter emergency medicine, Scene times, Ultrasound

\section{LO73}

Exploring computed tomography of abdomen/pelvis usage across emergency departments in the Calgary zone

K. Ukrainetz, S. Vatanpour, PhD, J. Elzinga, MD, S. Dowling, MD

University of Calgary, Calgary, $\mathrm{AB}$

Introduction: Computed tomography abdomen/pelvis (CTAP) is one of the most frequently ordered scans by physicians in the emergency department (ED). Concerns were raised by our radiology group as an $8 \%$ average annual increase in CTAP utilization had been noted albeit this data was not adjusted for ED volumes or patient factors. The objectives of this study were to explore the trends in CTAP usage over the last 5 years with respect to year over year changes (adjusting 
for ED patient volumes) in order to gain a better understanding of what factors contribute to the increasing rates of CTAP usage and to compare outcomes during the study period.

Methods: This electronic health records study included all patients that underwent a CTAP at any of the four Calgary EDs between 2014 and 2019. The data was analyzed both overall and by year/site and adjusted for ED volumes to determine if CTAP usage had changed during the study period. Confounders such as patient characteristics (age, CTAS, arrival mode) and patient outcomes (revisit rate at $72 \mathrm{~h}$, consultation and rate of admission) were also analyzed. Specific CT modalities were explored in order to determine if certain modalities were contributing to the disproportionate increase in CTAP usage. Statistical analysis was conducted using both the Chi-square and linear regression depending on the nature of the variable.

Results: The rate of CTAP usage in Calgary ED's increased from $4.9 \%$ in 2014 to $6.3 \%$ in 2019 (difference $1.4 \%$, p value $=0.01$ ). Similar trends were observed in CTAP usage across all four sites. During the same time period, patients who underwent CTAP were older (median age of 53.15 in 2014 vs 57.16 in 2019, ( $p=<0.0001$ ), had a higher proportion of CTAS $1 / 2$ (42.66 in 2014 vs 45.95 in 2019, $(\mathrm{p}=<0.0001)$, higher admission rates $(38.5 \%$ vs $40.23 \%$, $\mathrm{p}=<0.0001)$ and lower readmission rates $(2.93 \%$ vs $2.48 \%$, $\mathrm{p}=<0.0001)$.

Conclusion: During the 6 year study period, a statistically significant increase in annual CTAP rates across Calgary emergency departments was observed. Although the rate of CTAP increased, patients undergoing CTAP appeared to be sicker as indicated by the increased age, CTAS scores and admission rates. Furthermore, increasing CTAP was associated with lower $72 \mathrm{H}$ revisit rates. This study highlights the need for interpretation of increasing ED resource usage in the context of ED volumes and patient factors prior to embarking on projects to reduce low value care.

Keywords: Abdomen/pelvis, Computed tomography, Patient volumes

\section{LO74}

Does IVC ultrasound independently predict fluid status in spontaneously breathing, undifferentiated hypotensive patients? SHoC-IVC

R. Dunfield, MD D. Dutton, PhD, P. Ross, MD, J. Fraser, BN, D. Lewis, MBChB, P. Boreskie, MD, C. Pham, MD, S. Alrobaian, MD, F. Scheuermeyer, MD, K. Chandra, MD, P. Atkinson, BSc, $\mathrm{MBChB}, \mathrm{MA}$

Dalhousie University/Saint John Regional Hospital, Saint John, NB

Introduction: Accurately determining the fluid status of a patient during resuscitation in the emergency department (ED) helps guide appropriate fluid administration in the setting of undifferentiated hypotension. Avoiding fluid boluses in overfilled patients is desirable. Our previous work has shown the feasibility of point of care ultrasound (PoCUS) for inferior vena cava (IVC) measurement as a potential noninvasive, rapid, repeatable investigation. Our goal was to determine the diagnostic utility of PoCUS IVC size and collapsibility in predicting fluid status in spontaneously breathing hypotensive ED patients.

Methods: We report a planned secondary analysis of data from SHOC-ED, a prospective RCT investigating PoCUS in patients with undifferentiated hypotension in 6 EDs in North America and South Africa. Adult ED patients with undifferentiated hypotension as determined by sustained systolic blood pressure $<100 \mathrm{mmHg}$ or shock index $>1.0$ were included. We prospectively collected data on IVC size and collapsibility for 129 patients in the POCUS group using a standard data collection form, and independently assigned a fluid status (overfilled, normal, underfilled) from a composite clinical assessment blinded to POCUS findings. The primary outcome was the diagnostic performance; i. e. sensitivity, specificity and area under the curve (AOC); of IVC characteristics on PoCUS in the detection of an overfilled fluid status.

Results: 125 of 129 patients had successful final fluid status determination of which 107 were classified as underfilled, 13 normal, and 7 overfilled. A receiver operating characteristic (ROC) curve was plotted using several IVC size and collapsibility categories. The best overall performance utilized the combined parameters of a dilated IVC $(>2.5 \mathrm{~cm})$ with poor collapsibility (less than $50 \%$ ) which had a sensitivity of $85.7 \%$ and specificity of $86.4 \%$ with an area under the curve (AOC) of 0.924 for predicting an overfilled fluid status.

Conclusion: Having previously reported that performing IVC PoCUS is feasible in spontaneously breathing hypotensive patients, we now report moderate sensitivity and specificity for IVC PoCUS as an independent predictor of an overfilled fluid status in patients with undifferentiated shock.

Keywords: Hypotension, Inferior vena cava, Point-of-care ultrasound

\section{LO75}

Is point-of-care ultrasound a reliable predictor of outcome during traumatic cardiac arrest? A systematic review and metaanalysis from the SHoC investigators

E. Lalande, MD, T. Burwash-Brennan, MD, K. Burns, MD, T. Harris, MBA, S. Thomas, MBA, M. Woo, MD, P. Atkinson, MBA

Université Laval, Centre Hospitalier de l'Université Laval (CHUL), Quebec, QC

Introduction: Point of care ultrasound (POCUS) has been shown to assist in predicting outcomes in cardiac arrest in general. We evaluated the test characteristics of POCUS in predicting return of spontaneous circulation (ROSC), survival to hospital admission (SHA), survival to hospital discharge (SHD) and neurologically intact survival to hospital discharge (NISHD) in adult and pediatric blunt and penetrating traumatic out-of-hospital or emergency department cardiac arrest patients.

Methods: We conducted a systematic review and meta analysis using PRISMA guidelines. We searched Clinicaltrials.gov, CINAHL, Cochrane library, EMBASE, Medline and the World Health Organization- International Clinical Trials Registry from 1974 to November 9, 2020. We included English and French studies. Risk of bias was assessed using the QUADAS-2 tool. We used a randomeffects meta-analysis model with $95 \%$ confidence intervals with Isquared statistics for heterogeneity.

Results: We included 8 studies involving 710 cases of traumatic cardiac arrest. Overall, survival rates were low. For all blunt and penetrating traumatic cardiac arrest, the specificity for ROSC (the proportion of patients with cardiac activity on POCUS who achieved ROSC) was $98 \%$ (95\% CI 0.13-1.0). The sensitivity (the proportion of patients with cardiac standstill on POCUS who failed to achieve ROSC) was $91 \%$ (95\% CI 0.67-0.98). The X exceeded 50\% indicating a substantial level of heterogeneity.

Conclusion: ROSC is extremely rare when cardiac standstill is identified on POCUS. Trauma patients in cardiac arrest, without an immediate indication for remediation of a reversible cause such as tension pneumothorax, or cardiac tamponade, should be assessed by cardiac point of care ultrasound, with strong consideration of termination of resuscitative efforts if cardiac standstill is confirmed.

Keywords: Point-of-care ultrasound, Prognosis, Traumatic arrest 


\section{Moderated poster}

\section{MP1}

Trends in computer tomography ordering in Calgary emergency departments

J. Elzinga, BSc, MD, C. Weaver, BSc, D. Wang, MSc, S. Dowling, MD

University of Calgary, Calgary, $\mathrm{AB}$

Introduction: While computed tomography (CT) is an important diagnostic tool in the emergency department (ED), evidence shows increasing CT use at many centres despite associated risks and costs. Despite concerns that CT use was increasing in the Calgary zone's EDs, the types of CT scans which were contributing most to this trend were unknown. The objective of modalities this project was to quantify changes in CT use at Calgary zone EDs from 2014 to 2019 and identify which CT were driving the increase in usage. These modalities could then be targeted for future quality improvement initiatives.

Methods: A population-based analysis of electronic medical records was performed for all ED visits at adult hospitals in Calgary, Alberta from 2014 to 2019. CT rates per 100 ED visits were compared between 2014 and 2019 using Poisson confidence intervals. Patient characteristics such as age and CTAS scores were assessed as potential contributors to variations in CT usage. The proportion of patients discharged and referred to a consultation service were analyzed as outcome measures.

Results: A total of 259,630 visits with a CT scan were identified in the study period. From 2014 to 2019 , CT scans per 100 ED visits increased from 15.7 to 19.3 ( +3.67 per 100 visits; relative $+23.5 \% ; \mathrm{p}<0.001)$. The three modalities with the largest absolute rate increases were CT Abdomen and Pelvis $(+1.19$ per 100 visits; relative $+26.6 \%$; $\mathrm{p}<0.001)$, CT Head and Neck Angiogram (+ 0.88 per 100 visits; relative $+113.4 \% ; \mathrm{p}<0.001)$ and $\mathrm{CT}$ for Pulmonary Embolism $(+0.71$ per 100 visits; relative $+62.19 \% ; \mathrm{p}<0.001)$. Together, these three accounted for $75.7 \%$ of the total increase in CT ordering. There were increases in mean patient age (absolute +2.7 years; $p<0.001$ ) and proportion of CTAS 1 and 2 patients (absolute $+0.8 \%$; $\mathrm{p}<0.001)$. The proportion of patients with a consultation increased (absolute $+3.2 \% ; \mathrm{p}<0.001$ ) over the study period. However, the percent discharged after imaging also increased (absolute $+0.5 \%$; $\mathrm{p}<0.001)$.

Conclusion: CT use in ED patients increased substantially in our region over the last 5 years, although during the same time periods patients were older, had higher acuity and a higher rate of consultation. The increased CT use has been largely driven by three CT modalities: CT Abdomen and Pelvis, CT Head and Neck Angiogram and CT for Pulmonary Embolism. These should be studied further for the appropriateness of their use and as targets for resource stewardship initiatives.

Keywords: Computed tomography, Ordering practices, Resource stewardship

\section{MP2}

The impact of performance feedback reports on physician ordering behaviour in the use of computed tomography pulmonary angiography

L. Salehi, MD, MPH, H. Yu, MD, J. Jaskolka, MD, M. Ossip, MD, P. Phalpher, MD, R. Valani, MBA, MD, M. Mercuri, MSc, PhD

William Osler Health System, Brampton, ON
Introduction: The increased utilization, and potential overutilization, of Computed Tomography Pulmonary Angiography (CTPA) is a well-recognized issue within emergency departments (ED), and one with few clear and effective solutions. Individualized performance feedback is one potential strategy with intuitive appeal and somemixed-evidence for efficacy in impacting physician behaviour. The objective of this study is to determine the impact of performance feedback reports on CTPA ordering behaviour among ED physicians. Methods: Beginning in September 2019, individualized reports containing detailed feedback on each physician's CTPA ordering behaviour were distributed every 6 months to all ED physicians at our institution's two EDs. We tracked individual and group ordering behaviour through each 6-month period. Our baseline pre-intervention period was January 1st to December 31st, 2018. Our primary outcomes are impact on (1) overall group ordering rate (defined as the total number of CTPAs divided by the total number of ED visits) and (2) overall diagnostic yield (total number of CTPAs positive for PE divided by the total number CTPAs ordered). Secondary analysis was done to determine the impact on those physicians with the highest CTPA utilization rate, defined as those in the highest quartile for ordering rate during the pre-intervention period. Our interim analysis examines the difference in outcome measures for the 6-month period of January 1 and June 30th 2020 with those of our pre-intervention period.

Results: Within the ED physician group, we observed a statistically significant increase in both diagnostic yield, from 11.7 to $15.2 \%$ $(\mathrm{p}<0.05)$ and in CTPA ordering rate, from 5.3 CTPA/1000 ED visits to $6.4 \mathrm{CTPA} / 1000 \mathrm{ED}$ visits $(\mathrm{p}<0.01)$ when compared to the baseline period. Within our highest utilizers, we saw a trend towards increased ordering rate (from 9.9 CTPA/1000 ED visits to 10.3 CTPA/1000 ED, $\mathrm{p}=0.74)$ and increased diagnostic yield $(10.3 \%$ to $13.9 \%, p=0.24)$ though neither reached statistical significance.

Conclusion: The interim results of this study demonstrate some encouraging trends in physician diagnostic yield that coincided with the study period. However, we suspect that the impact of the COVID19 pandemic, and the resultant decrease in ED volumes, particularly of low-acuity patients, played a large part in influencing these results. This points to a potentially greater impact of environmental and institutional factors on physician ordering behaviour.

Keywords: Computed tomography angiography, Pulmonary embolism, Quality improvement

\section{MP3}

Use of an instant messaging platform to improve communication between the emergency department and medical imaging after hours

J. Choi, MD, MPH, D. Wang, MD, E. Bartlett, MD, MPH, H. Schmidt, MD

University Health Network, Toronto, ON

Background: There is frequent communication between the emergency physician (EP) and the radiology resident (RR) on-call after regular working hours. However, frequent phone calls interrupts workflow, decreases efficiency, increases provider frustration and the potential for error. To improve communication processes, we explored the use of a secure instant messaging platform to observe its effects on after-hours communication between the EP and RR on-call. We aim to have a response time of less than $10 \mathrm{~min}$ for $80 \%$ of messages sent from the EP to the RR within 3 months using the instant messaging platform. This is compared to the current institutional policy to return pages within 10 min of receipt.

Aim statement: This proof of concept project used Microsoft Teams, a productivity platform with instant messaging functions that was 
approved for clinical use at our tertiary care academic teaching hospital. EPs had the option to message the RR on-call via MS Teams during the after hours period (outside of 0800-1700, Monday-Friday) for non-emergent questions and consultation. Response times were recorded and analyzed. Additionally, surveys were sent to participating EPs to gather feedback and perceptions of the new workflow. Measures and design: Between July 1-Sept 30 2020, 16 EPs and 28 RRs participated in the pilot project. A total of 458 messages were sent from the EP to the RR during the after-hours period. The median response time was $1 \mathrm{~min}$ (25th percentile $=<1 \mathrm{~min}$, 75th percentile $=5 \mathrm{~min}$, range $0-80 \mathrm{~min}$ ). $94 \%$ of messages had a response time less than $10 \mathrm{~min}$ and $73 \%$ of messages had a response time of less than $5 \mathrm{~min}$. Approximately $2 \%$ of messages had no response. The survey sent to the participating EPs demonstrated strong support for the initiative, with $80 \%$ preferring instant messaging compared to traditional paging.

Evaluation/results: The goal of $80 \%$ response rate within $10 \mathrm{~min}$ by the RR was achieved. We have demonstrated that communication using a PHI-approved instant messaging platform between the ED physician and RR for non-emergent issues is viable and effective, allowing for rapid response between the two parties. Further encouragement and engagement of instant messaging use is likely to decrease reliance on traditional pagers and switchboards, and potentially improve user workflow efficiency and provider satisfaction.

Keywords: Clinical workflow, Instant messaging, Quality improvement and patient safety

\section{MP4}

Impact of major health information technology failures on the emergency department

V. Homier, MD, MSc, H. Gangatharan, BMSc, M. Bouchard, M. Beique, BSc, MD

McGill University, Montreal, QC

Introduction: Despite the increased adoption of health information technology (HIT) in hospitals across Canada, few studies have investigated the challenges associated with major HIT failures. Amongst the most disruptive failures are downtime periods in the emergency department (ED) where efficiency and timeliness of clinical care are integral to patient safety and outcomes. We aimed to quantify the impact on ED flow parameters, medical documentation and laboratory turnaround times (TAT) during downtime periods impacting both the Montreal General and Royal Victoria hospitals.

Methods: Downtime periods occurred on June 26, 2018 and September 30, 2019 lasting $9 \mathrm{~h}$ and $5 \mathrm{~h}$ respectively. A review of the electronic medical records (EMR) and laboratory results were done for visits during the downtimes. Of the 167 patients registered during these periods, 3 patients were excluded for inaccurate registration times providing a sample size of 164 visits. Laboratory TAT and ED flow parameters (length of stay (LOS), time to MD management, number of patients who left without being seen (LWBS), against medical advice (AMA), or were reoriented) were collected for all patients. Data on physician documentation presence and time to medication administration were collected only on patients seen by a physician during downtimes. Flow parameters were compared with the 2018-2019 yearly averages. For comparison of all other variables, the same metrics were assessed for 230 patients that registered during time periods exactly 1 -week prior to the IT failures.

Results: For statistical analysis, a univariate analysis was conducted with a significance level of $<0.05$. No difference was found in ED length of stay but time to MD evaluation increased by $49 \mathrm{~min}$. There was a significant increase in patients that left AMA (4.9\% vs $0.7 \%$ ) but no difference in those that LWBS or were reoriented. Laboratory
TAT increased during downtimes; CBC by 109 min, metabolic panel by $95 \mathrm{~min}$, Troponin by $148 \mathrm{~min}$, venous blood gas by $52 \mathrm{~min}$. A significant number of patients had missing physician notes during downtime ( $27 \%$ vs $2.5 \%)$. Although not statistically significant, time to medication administration from initial evaluation increased by 76 min.

Conclusion: Downtimes had a significant impact on emergency physician documentation and laboratory turnaround times. The number of patients that left AMA also increased significantly. Future studies and training programs should target interventions that mitigate the consequences of downtimes.

Keywords: Downtime, Electronic medical records, Information technology failure

\section{MP5}

Transitions in health care states for frequent and non-frequent users of emergency departments: a population-based retrospective cohort study

P. McLane, PhD, A. Chen, MSc, M. Ospina, MSc, PhD, A. McRae, $\mathrm{MD}, \mathrm{PhD}, \mathrm{X} . \mathrm{Hu}, \mathrm{PhD}, \mathrm{R}$. Rosychuk, PhD

Alberta Health Services Emergency Strategic Clinical Network/ University of Alberta, Edmonton, AB

Introduction: Frequent users of emergency departments (ED) account for approximately $21-28 \%$ of ED visits and are a common focus of study. Using a multistate modeling approach, this study examined transitions in care among the ED, hospital, and community for frequent users. Our objective was to identify significant factors related to time spent in each care setting in order to better understand patient flow within the health care system.

Methods: This was a retrospective cohort study relying on population-based administrative health data from EDs and hospitals in Alberta and Ontario during fiscal year 2015/2016. Frequent users were defined as the top $10 \%$ of ED users $(n=579,229)$ while a random sample $(n=2,103,387)$ of all other users served as controls. Data on whether a patient had a regular primary care provider was available for Ontario but not for Alberta. Cox proportional hazards regression models were used to model care transitions adjusting for specific covariates.

Results: Transition times from community to ED were shorter for Alberta frequent users [adjusted hazard ratio (HR) 1.21, 95\% confidence interval (CI) 1.20, 1.21] than for Alberta controls (HR 1.09, $95 \%$ CI 1.08, 1.09) and for Ontario frequent users without a primary health care provider (HR 1.08, 95\% CI 1.07, 1.09) than controls (HR $0.95,95 \%$ CI $0.95,0.96)$. Once in the ED, transition time to hospital admission was longer for frequent users for males (frequent user: HR 1.06, 95\% CI 1.06, 1.07; control: HR 1.13, 95\% CI 1.12, 1.14) and Ontarians without a primary health care provider (frequent users: HR 0.84, 95\% CI 0.82, 0.85; controls: HR 0.96, 95\% CI 0.94, 0.98). Frequent users and controls had differing transition times among care settings for several diagnoses.

Conclusion: Lack of a primary health care provider in Ontario led to longer times between transition from community to ED for controls but shorter times for frequent users. Alongside other differential effects of predictors, results suggest that frequent users differ from other patients in important ways not captured by other variables in our models. Once admitted to hospital, frequent users did not necessarily have longer stays than controls. Study results provide a better understanding of transitions among care types for frequent users and insights on factors that could be modified to better serve patients across the health system. In addition, frequency of ED use could be considered as a predictor variable in future health system modelling studies. 
Keywords: Frequent users, Health systems, Transitions in care

\section{MP6}

Cost analysis of outpatient parenteral antibiotic therapy (OPAT) and inpatient strategies for emergency department patients with cellulitis

K. Yadav, MD, MSc, K. Thavorn, PhD, S. Kumar, MSc, S. Chhabra, MD, H. Rosenberg, MD, A. Nath, MD, R. Ohle, MD, MSc, K. Suh, $\mathrm{MD}$, D. Eagles, MD, MSc

Department of Emergency Medicine, University of Ottawa, Ottawa, ON

Introduction: Emergency department (ED) patients with non-purulent cellulitis requiring intravenous (IV) antibiotics may be treated via outpatient parenteral antibiotic therapy (OPAT) as opposed to hospitalization. The primary objective was to compare healthcare costs for the following treatment strategies: community IV antibiotics with referral to an OPAT clinic operated by infectious disease specialists ('OPAT clinic' strategy); community IV antibiotics with return to $\mathrm{ED}$ if necessary ('return to ED' strategy); and hospital admission.

Methods: Using a hospital administrative database, we conducted a cost analysis using patient-level data of adult patients presenting to two tertiary care EDs in 2015 with non-purulent cellulitis who were treated with IV antibiotics in one of three ways: OPAT clinic strategy; return to ED strategy; and hospital admission. The primary outcome was the mean total cost per patient for each treatment strategy. We also compared the incremental cost of the OPAT clinic strategy to the return to ED group and hospitalized patients. A secondary outcome was OPAT failure (hospitalization after $48 \mathrm{~h}$ of OPAT). Regression analyses were performed using a generalized linear model adjusted for baseline differences in age, sex and comorbidities.

Results: A total of 808 patients met inclusion criteria: OPAT clinic strategy $(\mathrm{N}=341$, mean age 61.7 years, $59.8 \%$ male); return to $\mathrm{ED}$ strategy $(\mathrm{N}=228$, mean age 59.0 years, $49.1 \%$ male $)$ and hospital admission ( $\mathrm{N}=239$, mean age 65.0 years; $51.9 \%$ male). The mean total cost of care for the three treatment strategies were: OPAT clinic: \$2164 (SD \$1678); return to ED: \$1421 (SD \$2302); and hospital admission: \$10,701 (SD \$12,757). Results from the regression analysis suggested that the OPAT clinic strategy was associated with a cost-saving of \$7394 (95\% CI \$6328-\$8460, p < 0.001) compared to hospital admission. When comparing the two outpatient strategies, the OPAT clinic group was associated with an increased cost of $\$ 651$ (95\% CI \$307-\$995, p < 0.001) when compared to the return to ED approach. OPAT failure occurred in $2.3 \%$ for the OPAT clinic strategy and $2.6 \%$ for the return to ED strategy.

Conclusion: This is the first Canadian study that shows the cost of different OPAT strategies for patients with cellulitis. While both OPAT strategies are safe and far less costly than hospital admission, our findings suggest that a dedicated OPAT clinic for patients with cellulitis is more expensive.

Keywords: Cellulitis, OPAT

\section{MP7}

Rating the trustworthiness of emergency medicine clinical practice guidelines with the NEATS instrument

S. Upadhye, MD, MSc, J. Yan, MD, MSc, G. Ghate, MD, MSc, J. Morris, MD, MSc, S. Ananthanarayanan, MD, MPH, M. Dingwall, MD, P. Kapend, MD, C. Davies-Schinkel, MSc, RN, R. Valani, MBA, MD
McMaster University, Hamilton, ON

Introduction: It is imperative that clinical practice guidelines (CPGs) be "trustworthy" for use in clinical practice (Institute of Medicine 2011). The National Guideline Clearinghouse Extent of Adherence to Trustworthiness Standards (NEATS) rating tool was created to assess CPG adherence to IOM standards (2019). The goal of this study was to apply the NEATS rating tool in appraising the trustworthiness of emergency medicine (EM) guidelines.

Methods: Seven EM raters (community, academic) were recruited and trained in the use of the NEATS instrument. Twenty current CPGs were retrieved from the American College of Emergency Physicians (ACEP) Clinical Policies website for rating. Raters were divided into 2 balanced groups, and each group rated 13 Policies (including 3 overlaps with other group) with the NEATS tool (15 specific questions). Raters also provided a global assessment of trustworthiness and ED implementability (derived from the AGREEII instrument). Individual ratings were pooled and analyzed for consensus scoring. Inter-rater reliability was calculated using generalizability theory, and the optimal number of raters determined using a D-study.

Results: ACEP Clinical Policies scored as follows with respect to NEATS domains: (1) "Strong" in Guideline group membership, Methods (Evidence reviews, Creating recommendations), and Updating plans, (2) "Moderate" in Conflict of Interest reporting, External review, and (3) "Low" in Patient/public involvement. Overall trustworthiness scores were high, and overall implementability scores improved over time. There were also improved Methods scores over time (since 2011). Inter-rater reliability (using G-theory) was 0.82 (95\% CI 0.73-0.89). The optimal number of raters in this application was 5 via D-study.

Conclusion: The NEATS rating instrument has high inter-rater reliability when used by various trained EM physicians to rate CPGs. ACEP Clinical Policies scored highly (or improved over time) in Guideline Group composition, Methods used and Updating plans. Moderate scores were noted for reported Conflict of Interests and External Review, and persistently Low scores for Patient/public involvement. Overall, these Policies have high trustworthiness ratings, and variable implementability scores. EM author groups can use the NEATS tool to ensure proper planning and execution of CPG development projects to maximize trustworthiness. More patient/ public involvement needs to be a priority in future EM CPG projects. Keywords: Clinical practice guidelines, Trustworthiness

\section{MP8}

Urban Alberta emergency department pediatric cannabis presentations: the legalization experience

M. Yeung, C. Weaver, BSc, R. Hartmann, MD, MSc, R. Saah-Haines, $\mathrm{PhD}$, E. Lang, MD

University of Calgary, Calgary, $\mathrm{AB}$

Introduction: Canada legalized non-medical cannabis possession and sale in October, 2018. In the United States, state cannabis legalization has been associated with an increase in pediatric cannabis-related emergency department (ED) visits, however the impact of nationwide legislation on pediatric visits has not previously been reported in Canada. We identified changes in pediatric cannabis-related ED visits, and presentation patterns pre- and post-legalization in urban Alberta EDs.

Methods: Administrative data was queried for urban Alberta cannabis-related ED visits among patients $<18$ from October 1st, 2013 to February 29th, 2020. The population was split into children (0-11), younger adolescents (12-14) and older adolescents (15-17). Incident 
rate (IR) and relative risk (RR) ratios were calculated to identify changes pre- and post-legalization. The IR identified changes against the entire Alberta population, and was adjusted for population growth. The RR identified changes against the pre-legalization ED cohort, allowing for presentation pattern analysis.

Results: There were 91 children, 458 younger adolescents, and 1973 older adolescent visits in both study periods. We identified increasing cannabis-related ED visits in children (IR 2.51, 95\% CI 1.62-3.88) and unintentional ingestions in children (IR $1.77,95 \%$ CI $1.42-2.20$ and RR 1.24, 95\% CI 1.05-1.47). We also identified increased unintentional ingestions in older adolescents (IR 1.36, 95\% CI 1.071.71 and RR $1.48,95 \%$ CI 1.21-1.81). There was no change to incidence rate in younger adolescents (IR 1.18, 95\% CI 0.95-1.45) and older adolescents (IR 0.92, 95\% CI 0.82-1.01). Presentation patterns remained similar, though older adolescent co-ingestant use decreased (RR 0.77 , 95\% CI 0.67-0.88) while hyperemesis cases increased (RR 1.64, 95\% CI 1.13-2.37). Notably, there were low sample sizes of children presenting with seizure symptoms (pre-legal $\mathrm{n}=13$, post-legal $\mathrm{n}=6$ ) and admitted to critical care (pre-legal $\mathrm{n}=5$, post-legal $\mathrm{n}=3$ ). Results for children are tempered by relatively low sample sizes and translate to 1.49 visits per month in an area with a population exceeding 2 million.

Conclusion: We demonstrate cannabis legalization was associated with an increase in child and older adolescent unintentional cannabis ingestion rates, as well as increased child cannabis-related ED visits. Changes highlight need for ongoing messaging surrounding accidental pediatric exposures, particularly in children and older adolescents.

Keywords: Cannabis ingestions, Legalization, Pediatrics

\section{MP9}

Sentinel visits in pediatric emergency department patients with diabetes mellitus as a warning sign for hyperglycemic emergencies

B. Maxwelll, BSc, MD, N. Samarasinghe, B. Vujcic, BSc, MSc, M. Columbus, PhD, K. VanAarsen, MSc, J. Yan, BSc, MD, MSc

\section{Western University, London, ON}

Introduction: Diabetes mellitus is the most common endocrinologic disorder in Canadian pediatric patients. Patients may have a sentinel visit (defined as an emergency department (ED) visit within the preceding 14 days) for a precipitating reason prior to presenting again to the ED with hyperglycemia or diabetic ketoacidosis (DKA). Little research has been done describing the outcomes of these types of sentinel visits in the pediatric population. The objective of this study is to describe the epidemiology and outcomes of pediatric patients presenting with a sentinel ED visit prior to their visit for a hyperglycemic emergency.

Methods: We conducted a retrospective chart review on patients $<$ 18 years old presenting to one of three tertiary care EDs between Jan 2014 and Sept 2019 with a discharge diagnosis of hyperglycemia, DKA, or diabetes. Cases were identified by health records review of agreed-upon diagnoses based on ICD-10 codes. Research personnel reviewed electronic medical records and collected information on demographics, management and disposition for that visit, as well as for any ED visits in the last 6 months. Descriptive statistics were used to present the data.

Results: A total of 342 patients were identified. Data on 245 patients was available at the time of abstract submission. We excluded 17 cases for not fitting the proper discharge diagnosis inclusion criteria and 11 due to being 18 years old. Of the 217 included cases, mean (SD) age was $11.5(4.4)$ and $57 \%$ were female. There were 125 $(57.6 \%)$ with a known history of diabetes. There were $24(11 \%)$ patients who had a sentinel ED visit within the preceding 14 days. Of these patients, 7 (29\%) had hyperglycemia and $6(25 \%)$ were diagnosed with an infectious illness. During the sentinel visit, 22 (91.6\%) patients were discharged while $2(8 \%)$ were admitted. When subsequently returning to the ED following a sentinel visit, $14(58 \%)$ patients were admitted with $3(12.5 \%)$ being admitted to an intensive care unit.

Conclusion: In pediatric patients presenting to the ED with a hyperglycemic emergency, $11 \%$ had a sentinel visit within the preceding 14 days. It was common for patients with a sentinel visit to require admission to hospital during their subsequent $E D$ visit. These sentinel visits may serve as an opportunity for patient education, improved discharge instructions and modification of risk factors to help prevent the development of future hyperglycemic emergencies. Keywords: Diabetes, Hyperglycemia, Sentinel visit

\section{MP10}

Treatment of alcohol withdrawal syndrome in the emergency department: a rapid review

J. Koh, MD, MPH, M. Malczewska, BA, M. Doyle-Waters, MA, MLIS, J. Moe, MD, MSc, MD, MSc, MA

College of Medicine, University of Saskatchewan, SASKATOON, SK

Introduction: People who experience alcohol-related harms often seek care in the emergency department (ED). In Canada, there is an ongoing rise in ED visits due to alcohol withdrawal. Published clinical guidelines on alcohol use disorder recommend stratifying patients with alcohol withdrawal based on their risk of developing complications such as generalized tonic-clonic seizures or delirium tremens. These guidelines are largely limited to primary care and outpatient settings, and do not provide specific guidance for ED providers. We aimed to address this gap by synthesizing evidence on the treatment of alcohol withdrawal syndrome in the ED.

Methods: We conducted a rapid review by searching MEDLINE, Embase, and CENTRAL (1980-2020). We searched for grey literature on Google and hand-searched the conference abstracts of relevant addiction medicine and emergency medicine professional associations. We included interventional and observational studies that reported outcomes of clinical interventions that aimed to treat alcohol withdrawal syndrome in adult ED patients. We assessed for bias using Cochrane risk-of-bias tools.

Results: We identified 13 studies that met inclusion criteria for our review (7 randomized controlled trials and 6 observational studies). Most studies were at high/serious risk of bias. We divided studies into four intervention groups and summarized evidence narratively: (1) benzodiazepines alone; (2) combined phenobarbital and benzodiazepines; (3) phenobarbital alone; and (4) phenytoin alone. Benzodiazepines decrease alcohol withdrawal seizure recurrence, but no clear evidence supports the use of one benzodiazepine over another in the ED. It is unclear if symptom-triggered benzodiazepine protocols are effective for use in the ED. More evidence is needed to determine if phenobarbital, with or without benzodiazepines, can be used safely and effectively to treat alcohol withdrawal in the ED. Phenytoin does not have evidence of effectiveness in preventing withdrawal seizures in the ED.

Conclusion: Few studies have evaluated the safety and effectiveness of guideline-supported treatments specifically in the ED setting. Benzodiazepines are the most evidence-based treatment for alcohol withdrawal in the ED setting. Pharmacotherapies that have demonstrated benefit for treatment of alcohol withdrawal in other inpatient and outpatient settings, (e.g., gabapentin, clonidine, and carbamazepine) need to be evaluated in the ED setting before routine use. 
Keywords: Alcohol, Withdrawal

\section{MP11}

Quality improvement opportunities in the care of emergency department patients presenting with mental health concerns and alcohol intoxication: a multicenter analysis

S. George, BHSc, MSc, B. Kelliher, MD, K. Lonergan, BSc, E. Lang, MD, MD CM

University of Calgary, Calgary, AB

Introduction: Mental health $(\mathrm{MH})$ presentations are on the increase in Canadian Emergency Departments (ED). Alcohol (EtOH) consumption is often a factor in mental health presentations to the ED. Consumption of alcohol by patients in this context presents a confounding factor that may affect care. The purpose of this study is to examine practice variations related to EtOH status and Mental Health presentations across four adult Emergency Departments in Calgary, Alberta, Canada.

Methods: We performed a comparative analysis of administrative data from Alberta Health Services (AHS) at urban Calgary Zone EDs from 2015 to 2019, examining the effect of EtOH status on rates of ED use by $\mathrm{MH}$ patients, rates of MH consults, admissions, and readmissions to psychiatry within 7 days of the index visit. An odds ratio was performed with $95 \%$ confidence intervals.

Results: 90,730 MH visits were included. 22,923 (25.3\%) of $\mathrm{MH}$ patients were EtOH positive. Of the ETOH positive patients, 5719 $(24.9 \%)$ received a psychiatric (psych) consultation. 29,296 (43.2\%) of EtOH negative patients received a psych consult. $32.5 \%$ of patients who were EtOH negative were admitted to psychiatry, while $16.7 \%$ of patients who tested EtOH positive were admitted to psychiatry. EtOH positive patients were less likely to receive a consultation (OR 0.44, 95\% CI $0.42-0.45$ ) or be admitted to psychiatry (OR $0.27,95 \%$ CI $0.25-0.29$ ). They were also less likely to be admitted if they represented within 7 days of their index visit $(0.36,95 \%$ CI $0.30-0.42)$. This trend was independent of CEDIS complaint description.

Conclusion: Positive EtOH status in the ED is significant confounding factor to patients' care in the ED and generally reduces the odds of involvement by psychiatry. These data highlight areas for further study into the impact of a positive EtOH test on care of $\mathrm{MH}$ patients in the ED. The lower likelihood of admission and consultation, also points to the need for more community based EtOH services for patients which can further reduce the burden on EDs and the healthcare system.

Keywords: Alcohol, Mental health, Quality improvement and patient safety

\section{MP12}

Overdose and naloxone dosing trends at Insite supervised injection facility in British Columbia: a retrospective cohort study

R. Cho, BSc, MD, R. Purssell, MD, R. Joe, MBChB, MSc, Y. Wang, MSc, F. O'Sullivan, BA, PhD, K. Lin, BMSc, A. Rowe, BSc, MD, J. Moe, MD, MSc, MA

University of Toronto/Sunnybrook Health Sciences Centre, Toronto, ON

Introduction: British Columbia (BC) is at the centre of the opioid epidemic that has been exacerbated by the emergence fentanyl and other ultra-potent opioids in 2015. Recent evidence suggests that higher doses of naloxone may be required to reverse toxicity caused by fentanyl. However, guidelines for naloxone dosing are variable and were mostly based on expert opinion prior to the emergence of illicit ultra-potent opioids. Therefore, we aimed to understand trends in opioid overdose, naloxone dosing, and overdose reversal over time at Insite, a community supervised injection facility in Vancouver, BC. Methods: We performed a retrospective cohort study of patients with opioid overdose at Insite. We evaluated the yearly trends in the total number of overdoses seen at Insite, naloxone doses used and proportions successfully reversed, with particular attention to pre- and post-2015, the emergence of fentanyl in BC. We used non-parametric median tests to compare doses and Chi-square tests to compare proportions reversed. We also compared naloxone doses among patients who were transferred to hospital, refused transfer and not offered transfer.

Results: There was an increase in the number of community overdoses managed by Insite clinicians from 2014-2015 $(\mathrm{n}=586)$ to 2016-2017 ( $\mathrm{n}=2033)$. However, cumulative naloxone doses administered by Insite clinicians were similar in 2014-15 and in 2016-2017 [median 0.4 mg (IQR 0.4-0.8 mg) for both, $\mathrm{p}=0.21$ ]. Fewer patients were successfully reversed after December 31, 2015 (44.1\% in $2016,29.7 \%$ in 2017 ) than before $(47.8 \%$ in $2014,55.1 \%$ in 2015) ( $\mathrm{p}<0.001)$. Cumulative naloxone doses administered were higher in patients who were offered hospital transfer [median $0.8 \mathrm{mg}$ (IQR $0.4-0.8 \mathrm{mg}$ )] compared to patients who were not offered transfer [median $0.4 \mathrm{mg}$ (IQR 0.4-0.8 mg)] ( $<<0.001)$.

Conclusion: Overdose numbers at Insite increased markedly after the emergence of illicit fentanyl in 2015. Naloxone doses administered by Insite clinicians remained similar over time but fewer patients were successfully reversed after December 31, 2015 than prior to this date. Keywords: Fentanyl, Naloxone, Overdose

\section{MP13}

Relationship of community pharmaceutical and opioid prescribing to the opioid epidemic

G. Innes, MD, MSc, J. Hayward, MD, MSc

University of Calgary, Calgary, $\mathrm{AB}$

Introduction: Opioid related overdose deaths increased dramatically in Canada between 2016 and 2019, now approaching 18,000. The opioid crisis has been attributed in part to physician opioid prescribing behaviour. Our goal was to study medication and opioid use by emergency department (EDs) patients over time. Our hypothesis is that temporal changes in the community prescription of opioids and other psychoactive drugs may correlate with rising opioid deaths.

Methods: This is a preliminary analysis of administrative data examining a random sample of ED patients who visited Alberta's 15 tertiary, urban and regional adult hospitals from 2011 to 2019. We excluded patients with cancer or palliative care, patients with preexisting opioid use disorders (OUD), pregnant patients and repeat ED visits. To characterize community medication/opioid prescribing, we queried the AHS pharmaceutical information network (PIN) database and collated all medications/opioids listed on the medication profiles of eligible patients. Our unit of analysis was the prescription. We summarized numbers and proportions of medications/opioids stratified by drug types, and documented trends over time. We calculated standardized mean differences to determine the significance of observed changes in prescribing patterns.

Results: Of 96,771 medications taken by eligible patients, the most common were psychoactive drugs $(28.6 \%)$, cardiovascular drugs $(20.5 \%)$, analgesics $(11.1 \%)$, endocrine-related drugs, gastrointestinal agents $(9.6 \%)$, antibiotics $(6.4 \%)$ and others. None of these categories increased or decreased significantly during the study years. Of 31,547 psychoactive drugs, $50.7 \%$ were antidepressants or mood stabilizers, $30.0 \%$ sedatives, $18.2 \%$ antipsychotics, and $1.1 \%$ other. Of 12,809 
analgesics prescribed, $41.3 \%$ were opioids, $19.7 \%$ NSAIDs, $15.7 \%$ acetaminophen, $11.6 \%$ gabapentinoids, $7.1 \%$ muscle relaxants, and $4.6 \%$ other. Rates and proportions of psychoactive, analgesic, and opioid prescription did not change significantly during the study period; however, between 2013 and 2018 there was a gradual $~ 25 \%$ decrease in the use of codeine preparations $(\mathrm{P}<0.001, \mathrm{SMD}=$ 0.475 ), which corresponded to a similar increase in the use of potent opioids (morphine, hydromorphone, oxycodone).

Conclusion: We found no change in the community use of psychoactive or opioid drugs over 8 years, but observed a substantial increase in prescriptions for potent opioids, which may have temporal correlation with the opioid crisis.

Keywords: Analgesics, Opioids, Overdose

\section{MP14}

A comparison of the management of patients with advanced cancer vs. other end-stage conditions in two Canadian emergency departments

S. Kirkland, MSc, E. Yang, MSc, M. Garrido Clua, MSc, M. Kruhlak, BSc, C. Villa-Roel, MD, PhD, A. Elwi, PhD, B. O’Neill, RN, MBA, S. Duggan, MD, A. Brisebois, MD, MSc, D. Stewart, MD, B. Rowe, $\mathrm{MD}, \mathrm{MSc}$

University of Alberta, Edmonton, AB

Introduction: It is common for patients with end-of-life (EOL) conditions to present to emergency departments (EDs), particularly those with advanced cancer. Improving our understanding of how patients with advanced cancer are managed in the ED can identify potential gaps in care and targets for intervention. The objective of this study was to compare the characteristics and ED management of patients who had advanced cancer with those who had other EOL conditions.

Methods: This prospective observational cohort study was conducted in two EDs in Edmonton, AB between March and August 2018. A modified screening tool was used by volunteer emergency physicians to identify adult patients with EOL conditions. Supplemental patient data were collected via chart review. Bivariate analyses for dichotomous and continuous variables were performed using Chi-squared $\left(\mathrm{X}^{2}\right)$ or Fisher's exact test and Wilcoxon signed-rank test or t test, as appropriate. Due to the exploratory nature of this analysis and the multiple statistical tests performed, a significance level was set at $\mathrm{p}<0.001$

Results: Among 663 patients with EOL conditions, 272 (41\%) presented with advanced cancer. Patients with advanced cancer were younger ( 71 years vs. 80 years; $p<0.001$ ), were less likely to arrive to the ED via EMS ( $45 \%$ vs. $70 \%, p<0.001$ ), and were less likely to present to the ED with goals of care $(53 \%$ vs. $75 \%$; p < 0.001$)$. Patients with advanced cancer were more likely to receive referrals to other services post-discharge $(38 \%$ vs $23 \% ; \mathrm{p}<0.001)$ and to have a history of ED visits $(77 \%$ vs. $64 \% ; \mathrm{p}<0.001)$ than patients with other EOL conditions. There were no significant differences between the groups in frequencies of consultations (80\% vs $75 \%$ ), invasive procedures (18\% vs $17 \%$ ), or admissions (63\% vs $66 \%$ ).

Conclusion: While some differences exist between patients with advanced cancer and other EOL conditions exist, both groups undergo significant investigations and consume valuable ED resources. Facilitation of early palliative care referrals might be one strategy to improve outcomes for patients with EOL conditions and unmet palliative needs, including those with advanced cancer. The impact of such approaches on important health outcomes and the quality of life of patients and families should be reported.

Keywords: Emergency department, Palliative care
MP15

Differences in cognitive processes and cognitive load in chest radiograph interpretation along the spectrum of medical expertise in emergency medicine

M. Morra, BSc, H. Braund, PhD, A. Hall, MD, MMed, A. Szulewski, MD, MHPE, PhD

Queen's University, Kingston, ON

Introduction: In the emergency department (ED), chest radiographs (CXRs) provide essential information for clinical diagnostic reasoning. Errors in radiograph interpretation are known to lead to negative patient outcomes. Emergency physicians are required to be proficient in CXR interpretation to identify important pathologies before formal interpretation by a radiologist. An understanding of the differences in cognitive processes and cognitive load (CL) in CXR interpretation along the spectrum of expertise in emergency medicine (EM) is warranted.

Methods: This study adopted a concurrent mixed methods research design. Participant groups included medical students (MS), junior (Jr) and senior (Sr) EM residents, and attending EM physicians (AP) in the ED at a tertiary care academic hospital. To elucidate cognitive processes, a cognitive task analysis was performed for each participant, which consisted of a think aloud protocol during the interpretation of a CXR of one of their patients while on shift in the ED. Interviews were audio recorded, transcribed verbatim, and coded for themes. The interview was followed by a quantitative questionnaire, where participants were asked to rate their CL, task complexity, distractibility, stress, and confidence level.

Results: Questionnaire data between groups were compared using Mann-Whitney U tests which revealed decreased levels of CL (MS vs $\mathrm{Sr}$ and MS vs AP: $\mathrm{p}=0.002$, Jr vs $\mathrm{Sr}: \mathrm{p}=0.004$, Jr vs AP: $p=0.005)$ and stress as experience level increased, higher confidence levels with increased experience, and no significant differences in task complexity or distractions between groups. Qualitative analysis of cognitive processes revealed four main themes during CXR interpretation: checking behaviour, information reduction, pattern recognition vs systematic viewing, and recognizing scope of practice. Participants with greater experience commonly utilized checking behaviour such as comparison to past radiographs, and effectively deprioritized task irrelevant data such as verbalizing fewer possible pathologies than less experienced participants. Experts also utilized a general overview technique as their initial viewing approach as opposed to a systematic viewing approach, and they better recognized an EM physicians' priorities in this task.

Conclusion: This study characterizes the differences in cognitive load and cognitive processes in EM personnel of varying levels of expertise during CXR interpretation, gaining insight into expertise development in this critical task.

Keywords: Chest radiograph interpretation, Cognitive load, Medical education

\section{MP16}

Successful habits in the age of competency-based medical education: a multicenter survey analysis

H. Alrimawi, MB BCh BAO, Q. Paterson, MD, S. Sample, MD, HBSc, M. Bouwsema, MD, BKin, O. Anjum, BSc, MD, M. Vincent, MSc, BMBS, R. Woods, MD, MMed, W. Cheung, MD, MMed, A. Hall, MD, MMed, L. Martin, MD, MHPE, T. Chan, MD, MHPE

McMaster University, Hamilton, ON 
Introduction: Competency-Based Medical Education (CBME) is a worldwide phenomenon. Emergency Medicine education in Canada was transitioned to a Competence by Design (CBD) framework in 2018. Entrustable professional activities (EPAs) are a major unit of analysis within $\mathrm{CBD}$, and residents are assessed upon completing these. Challenges have arisen since implementation limiting trainees from acquiring sufficient numbers of EPA observations. We conducted a multicentre survey to attempt to understand high performing situations and learn from the habits of the highest achieving faculty and residents.

Methods: We conducted a multicenter survey study examining enablers and barriers to EPA acquisition. The survey was sent via email to both faculty and residents of Emergency Medicine Programs at four centers in Canada (McMaster University, Queen's University, University of Ottawa, University of Saskatchewan). The design of the survey received ethical approval from both McMaster University and University of Saskatchewan. Surveymonkey was used to host the survey and it was piloted internally with the research team. The survey remained open for approximately 5 weeks and 2-3 reminders were sent to the participants at different centers. Interpretive description methods were used to analyze survey responses.

Results: In June-July 2020, 82 faculty members and 22 residents completed our survey. We identified 23 faculty members and 11 residents who described exceptionally high EPA observations in a single shift $(N \geq 4$; $\max =9)$. Habits of success for residents included: goal setting, matching EPAs to cases encountered throughout the shift; resident involvement to facilitate and make EPA completion easier; resident identification of EPAs; multiple rounds of EPA completion throughout the shift. Habits of success for faculty included: engaging in direct observations, interest in medical education; Familiarity with the resident (i.e. being a preceptor or a mentor); Time availability during the shift. Contextual factors that lead to high EPA acquisition included: eventful shifts with multiple resuscitations, opportunities to perform rare procedures; patient care volume during a shift allowing time for EPA observations.

Conclusion: There are several habits that can be adopted by faculty and residents to increase successful EPA acquisition during shifts in Emergency Medicine. Furthermore, contextual factors also have a role in determining the EPA experience of learners.

Keywords: Competency based medical education, Entrustable professional activity, Successful habits

\section{MP17}

Examining enablers and barriers to EPA acquisition using the theoretical domains framework: a qualitative framework analysis study

Q. Paterson, MD, H. Alrimawi, MB BCh BAO, S. Sample, MD, HBSc, M. Bouwsema, MD, BKin, O. Anjum, BSc, MD, M. Vincent, MSc, BMBS, R. Woods, MD, MMed, W. Cheung, MD, MMed, A. Hall, MD, MMed, L. Martin, MD, MHPE, T. Chan, BSc, MD, MHPE

University of Saskatchewan, Saskatoon, SK

Introduction: In 2018, the Royal College Emergency Medicine training program transitioned to the Competence by Design framework-a competency-based medical education program. Within this program, the major unit of assessment is the entrustable professional activity (EPA), an observable task of the discipline upon which workplace-based assessments are focused. However, with the shift to this new system, there are concerns that trainees are not getting enough opportunities for EPA observations. The purpose of this study was to identify enablers and barriers for accumulating EPA observations in emergency medicine.
Methods: We conducted a multicentre qualitative, interview-based study of both faculty and residents at four centres in Canada (McMaster University, Queen's University, University of Ottawa, University of Saskatchewan). After audio-recording and transcribing Zoom-based interviews by our trainee investigators, our team conducted a framework analysis of these data using the Theoretical Domains Framework (TDF; a model used to phenotype enablers and barriers towards a particular action). To decrease hierarchy and power differentials we ensured the investigators did not interview trainees or faculty from their own site. Each of the transcripts underwent line-byline coding looking for elements linked to known TDF codes by two independent coders. Codes which were deemed unclear were then reviewed by a second team of 4 coders to resolve differences.

Results: A total of 14 ( 8 faculty, 6 residents; $M=9, F=5$ ) participants were enrolled across the four sites. Interviews lasted between 26 and $62 \mathrm{~min}$ and yielded a total of 185 pages of transcripts. The most common TDF enabling codes were: behavioural regulation $(25.3 \%)$, memory, attention and decision processes $(16.3 \%)$, and knowledge (12.4\%). The most common TDF barrier codes were: environmental context and resources (29.5\%), beliefs about consequences $(21.5 \%)$, social influence $(8.1 \%)$, and goals $(8.1 \%)$.

Conclusion: EPA-focused assessment is likely to remain a key component of residency education for decades to come. Therefore, the enablers and barriers identified in this study may be useful for residency programs to create faculty or resident development, as well as identify systemic barriers that will need to be addressed.

Keywords: Competency based medical education, Entrustable professional activity, Theoretical domains framework

\section{MP18}

Unperceived learning needs among physicians caring for critically ill children in general emergency departments

A. Bdira, BSc, MD CM, MHSc, S. Reid, MD, F. Alnaji, MD, S. Tse, MD, L. Hayawi, MSc, R. Webster, PhD

University of Ottawa/CHEO (Children's Hospital of Eastern Ontario), Ottawa, ON

Introduction: Pediatric patients comprise a large proportion of acute care visits to Canadian general emergency departments (GED). GED physicians identify management of critically ill children as challenging and anxiety-provoking. There is limited literature assessing the unperceived learning needs among GED physicians caring for critically ill children. This study examined the types of pediatric emergencies that are most challenging for GED physicians and explored gaps in knowledge, skills or resources as identified by pediatric critical care (PCC) physicians who provide telephone (CritiCall) consultation.

Methods: An electronic cross-sectional survey was developed and piloted for this study. All PCC physicians who provide CritiCall consultations in Ontario were invited to participate.

Results: A total of 41 PCC physicians were surveyed and 23 participated $(56 \%)$. The top three patient age groups identified as most challenging for GED physicians to manage were neonates ( $<1$ month), infants (1-12 months), and toddlers (1-4 years). Among a list of options, respondents ranked the top three most challenging pediatric emergencies for GED physicians to manage as follows: non-arrest cardiac $(\mathrm{n}=16 / 23,70 \%)$, neurosurgical $(\mathrm{n}=13 /$ $23,57 \%)$, and cardiac arrest $(\mathrm{n}=10 / 23,44 \%)$. For non-arrest cardiac emergencies, $81 \%(13 / 16)$ reported that knowledge gaps, such as interpreting rhythm disturbances, were an issue "all/most of the time". For neurosurgical emergencies, 53\% (7/13) reported knowledge gaps, such as managing severe head injury, as an issue "all/most of the time". Challenges encountered "some of the time" during 
neurosurgical emergencies included difficulties with skills $(77 \%, 10 /$ 13) such as intubation, and lack of resources $(54 \%, \mathrm{n}=7 / 13)$ such as bedside tools. For cardiac arrest, resource limitations such as inadequate personnel were an issue "all/most of the time" $(50 \%, \mathrm{n}=5 / 10)$. Conclusion: Pediatric cardiac and neurosurgical emergencies, especially among infants and young children, are the most challenging for GED physicians to manage as reported by PCC physicians providing telephone consultation. Limited resources encountered outside of pediatric hospitals contribute to these difficulties. Educational interventions should focus on these clinical presentations with respect to diagnosis, management, resuscitation skills and provision of clinical resources. Planned key informant interviews will help to further delineate how best to address these learning needs.

Keywords: Continuing professional development, Critical care, Pediatric emergency medicine

\section{MP19}

Simulation-based teaching for rural and regional family medicine residents

\section{A. Yang, MD, C. Mara, MESc, K. Carter, MD}

London Health Sciences Centre, London, ON

Introduction: Family Medicine (FM) physicians in rural and regional hospitals often provide initial assessment and management of critically ill patients. However, many FM residents graduate without significant exposure to managing critical events. Simulations can increase opportunities to practice without risking harmful outcomes, and studies have shown low-fidelity simulations to be effective for knowledge transference in novice learners. We assessed whether lowfidelity simulation training during FM residency improves self-reported confidence in managing critical hospital events.

Methods: A convenience sample of first- and second-year residents in rural and regional FM programs at Western University was recruited in 2017-2018. A 3-h workshop was provided at Strathroy Middlesex Hospital using low-fidelity models to simulate critical events. Each resident led and assisted in various scenarios with subsequent debrief. Participants completed pre- and post-workshop surveys to measure career goals, prior simulation training, confidence in managing critically ill patients, and simulation satisfaction.

Results: Nine (53\%) first-year and $8(47 \%)$ second-year residents participated in the workshop. Common career goals included rural and regional practice $(n=14 ; 82 \%)$, FM clinic $(n=12 ; 71 \%)$, Emergency Medicine $(\mathrm{n}=8 ; 47 \%)$, and hospitalist $(\mathrm{n}=7 ; 41 \%)$. Most residents $(\mathrm{n}=13 ; 76 \%)$ received prior simulation training, including medical school workshops $(n=8 ; 47 \%)$, residency Emergency Medicine rotations $(\mathrm{n}=8 ; 47 \%)$, and resuscitation courses $(n=6 ; 35 \%)$. Most residents felt neutral $(n=6 ; 35 \%)$ or uncomfortable $(\mathrm{n}=7 ; 41 \%)$ managing critically ill patients before the workshop, whereas all residents $(\mathrm{n}=17 ; 100 \%)$ felt somewhat to very comfortable afterwards. Most residents felt neutral $(n=5 ; 29 \%)$ or uncomfortable $(\mathrm{n}=10 ; 59 \%)$ leading a medical team during a critical event before the workshop, whereas a majority $(n=15 ; 88 \%)$ felt somewhat to very comfortable afterwards. All residents $(n=17$; $100 \%)$ agreed that the workshop was relevant to their anticipated career needs, and most $(n=16 ; 94 \%)$ would change their practice management based on skills learned during the workshop.

Conclusion: A single, short, low-fidelity simulation workshop improved self-reported resident comfort levels in managing critically ill patients in hospital, including leading a medical team during resuscitation. The relative ease with which a low-fidelity simulation can be implemented should encourage future workshops in the residency curriculum.

Keywords: Family medicine, Rural, Simulation
MP20

Point-of-care-ultrasound (PoCUS) in undergraduate medical education (UGME): a scoping review of assessment methods

C. DeBiasio, BASc, A. Shefrin, MD, P. Pageau, MD, M. Woo, MD, W. Cheung, MD

University of Ottawa, Ottawa, ON

Introduction: Over the last 2 decades, point-of-care ultrasound (PoCUS) has rapidly expanded in undergraduate medical education (UGME). However, limited research exists examining which PoCUS assessment tools are being used in UGME. This scoping review aims to systematically determine the methods of assessment for PoCUS skills in UGME reported in the scientific literature.

Methods: Our protocol was developed using the Preferred Reporting Items for Systematic Reviews and Meta-analysis Extension for Scoping Reviews. We conducted a librarian assisted search of MEDLINE from inception through August 4, 2020. Inclusion criteria included all English Language UGME publications in which PoCUS related knowledge, skills, or behaviour were assessed in isolation from non-PoCUS related items. A single reviewer screened all abstracts for inclusion. A second reviewer independently screened a random sample of $10 \%$ of all abstracts for inclusion. A full review of all included articles was performed by a single reviewer and data was extracted to a standardized data collection form. Cohen's Kappa (к) was used to determine the agreement between the two reviewers. Miller's Pyramid of Assessment was used as a framework to categorize assessment tools.

Results: The search yielded 558 titles from 25 countries of which 166 articles were included for a full text review. $\kappa$ was $0.953 .84(51 \%)$ studies assessed clerkship students, whereas the remainder included pre-clerkship students or did not identify the level of training of participants. Retention was assessed in $88(53 \%)$ of the studies. Surveys of students' opinions, which did not objectively measure knowledge or behaviour, were used as the only assessment tool in 19(11.4\%) studies. $35(21 \%)$ articles used knowledge-based exams as the only assessment tool, with the majority (97\%) being multiple-choice questions. 111 $(66.9 \%)$ studies used behaviour-based practical exams where students had to demonstrate PoCUS skills. Of these, 22 (19.8\%) used a formal objectively structured clinical exam (OSCE) assessment. Both cognition and behaviour assessments, were used in 37 articles (22.2\%). 57 (34\%) studies claimed to assess for competency. Overall, only 4 $(0.02 \%)$ studies assessed for the highest level of Miller's pyramid.

Conclusion: This scoping review provides an overview of the PoCUS assessment methods reported in UGME literature and supports the need for standardization. Further study is required to determine the effectiveness of assessments in determining competency in PoCUS UGME.

Keywords: Assessment, Point-of-care ultrasound, Undergraduate medical education

\section{MP21}

Evaluation \& Earbuds: a program evaluation of the MacEmerg regional podcast

M. Khalid, BMSc, M. Bilic, BSc, M. Murdock, BMSc, M. Boulos, BSc, MSc, M. Mubasher, BSc, S. Sample, BHSc, MD, J. Dida, BSc, MD, MSc, B. Trotter, MD, T. Chan, BSc, MD, BEd, MHPE, K. Dong, $\mathrm{BSc}, \mathrm{MD}$

\section{McMaster, Hamilton, ON}

Introduction: 'MacEmerg Podcast' is a collaborative initiative between the three divisions of Emergency Medicine at McMaster University, with over 2500 total listens. With a large geographic 
distribution of faculty and trainees at academic and community hospitals spanning Niagara to Brampton, 'MacEmerg Podcast' acts as a vessel to share experiences, knowledge and insight. This study aimed to assess listening habits and examine voluntary feedback from listeners about the podcast's strengths and areas for improvement.

Methods: Listeners voluntarily participated in this ongoing crosssectional study, which began in June 2020. A 17-item survey with questions derived from the Revised METRIQ (rMETRIQ) scoring tool, quality indicators from the Delphi study, and open text questions, were used to gauge listener feedback. The survey link was visible on the podcast's website, show notes, and posted on the McMaster Emergency Medicine Twitter page with each episode release. Descriptive data and quantitative analysis using basic excel formulas assisted in the analysis of survey data.

Results: The survey had 30 participants with a 50\% completion rate. Incomplete responses were excluded. 93\% of participants were McMaster affiliated, with $47 \%$ being current staff, $33 \%$ residents, and $20 \%$ others, including medical students, nurses and more! On average, listeners listened to 7.40 episodes (13 total at time of survey distribution). $40 \%$ stated they were monthly listeners, 33\% felt they learned something new, $47 \%$ felt the content was useful to their practice, and $47 \%$ stated they would recommend the podcast to a colleague. Those who listened to at least one episode and answered the rMETRIQ scoring tool (7 questions each scored $0-3$, total $=21$ ) scored the podcast $16.7(\mathrm{SD}=4.7)$ on average. Participants remarked that the podcast's strengths include the breadth and novelty of information, applicability to practice, and local expertise on display. Potential areas of improvement include increased advertisement of the podcast and increased integration of summary statements.

Conclusion: Our survey results corroborate the strong degree of local support 'MacEmerg Podcast' has received thus far, and identify relevant strengths and potential areas of improvement. Next steps include contacting participants who expressed willingness for inperson interviewing to better understand listener perception. Future study modifications could include a larger sample size and incentivizing survey completion to increase participation.

Keywords: Medical education, Podcast, Podcast evaluation

\section{MP22}

\section{Examination of outcomes of ICU patients in Nova Scotia based} on location prior to ICU admission

R. Sadoway, BSc, MD, O. Loubani, BSc, MD

Dalhousie University, Halifax, NS

Introduction: Patients are admitted to the ICU from multiple areas of the hospital, including the emergency department, operating room, or medical or surgical ward. Patients admitted to ICU from different locations have heterogeneous mortality rates, possibly because of varying staff skill sets and disease states in different locations of the hospital. However, observed differences in mortality of ICU patients admitted from different locations may be due to differences in case-mix (diagnosis type, illness severity, etc.), which are not captured by examination of crude mortality figures. We sought to compare mortality of patients admitted to the ICU from different locations using modern statistical techniques to control for differences in case mix in the populations.

Methods: We conducted a retrospective review of data collected in the Nova Scotia (NS) provincial ICU database. We included all patients admitted to one of twelve adult ICUs in NS between April 1, 2018 and March 31, 2020. Case-mix adjusted mortality rate for patients admitted from each pre-ICU location using generalized mixed effects modeling. Pre-ICU locations examined were: bone marrow transplant unit, direct admit, emergency department, medicine floor, medicine intermediate care unit, obstetrics unit, operating room (OR), surgery floor, surgery intermediate care unit, and other. Case mix adjustment variables included admission diagnosis, age, sex, illness severity (measured using APACHE IV), preICU length of stay, and requirement for ventilation in the first $24 \mathrm{~h}$ of ICU stay.

Results: A total of 8461 ICU visits were examined. Patients admitted from medicine floor had a significantly higher than expected mortality after adjusting for case-mix, with an odds ratio for mortality of 1.581 (95\% CI 1.288-1.914) compared to other locations. Patients admitted from OR had a significantly lower than expected mortality after adjusting for case-mix, with an odds ratio for mortality of 0.643 (95\% CI 0.522-0.792) compared to other locations. No other locations were significant.

Conclusion: After adjusting for relevant case-mix features, patients admitted to NS ICUs from medicine floors have a higher-than-expected mortality, and patients admitted from the OR have a lower than expected mortality compared to other locations. Further study is required to determine what variables account for these differences in mortality. Medicine floors in NS should examine protocols for identifying acutely ill patients as earlier detection may improve outcomes. Keywords: Admission, Critical care, Outcomes

\section{MP23}

\section{Assessing mechanical ventilation practices in the emergency} department: a quality assurance study

S. Sharif, MD, S. Skitch, MD, PhD, B. Rochwerg, MD, MSc

\section{McMaster University, Hamilton, ON}

Introduction: Evidence from the Intensive Care Unit (ICU) supports the use of low tidal volume $(\mathrm{Vt})$ ventilation and normal oxygenation levels. However, there is limited literature on the uptake of these standards of care in the Emergency Department (ED). The objective of this study was to examine ventilation practices and clinical outcomes for adult patients who receive invasive mechanical ventilation in the ED.

Methods: This was a retrospective health records review conducted at a single academic centre in Ontario, Canada over a 1-year period. The following information was abstracted on pre-formatted case report forms: baseline demographics, comorbid conditions, ED length of stay, ED diagnosis, mode of ED arrival, indication for intubation, and ED ventilation settings. Lung protective ventilation (LPV) was defined as $\mathrm{Vt} \leq 8 \mathrm{ml} / \mathrm{kg}$ of ideal body weight and hyperoxia was defined as the provision of a percentage of inspired oxygen $\left(\mathrm{FiO}_{2}\right)>40 \%$ when saturations were $>95 \%$. The primary outcome was mortality during the index hospitalization. Secondary outcomes included the duration of mechanical ventilation among survivors. Categorical outcome variables were compared with the Chi squared analysis and continuous variables were compared with student's t test with a $\mathrm{p}$ value less than 0.05 being considered statistically significant. Results: Of the 126 patients in this study, $42 \%$ were female and the mean age was $65 \pm 18.3$ years. Only $20 \%$ of patients had an ideal body weight recorded in the ED and $40 \%$ of patients were ventilated with $\mathrm{Vt}>8 \mathrm{~mL} / \mathrm{kg}$. Furthermore, $48 \%$ of patients met criteria for hyperoxia while ventilated in the ED. Moreover, $31 \%$ of patients who received LPV died during the index hospitalization compared to $42 \%$ of patients who did not receive LPV ( $R R=0.75$; 95\% CI $0.47-$ $1.2)(\times 2=1.42 ; \mathrm{P}=0.43)$. Patients who met criteria for hyperoxia in the ED were more likely to die compared with patients who did not $(\mathrm{RR}=1.74,95 \%$ CI $1.04-2.92)(\times 2=4.71 ; \mathrm{P}=0.03)$. Amongst survivors, there was no difference in duration of mechanical ventilation amongst those receiving LPV versus those who did not $(\mathrm{t}=0.0$; $P=1.0)$. Likewise, there was no difference in duration of mechanical 
ventilation amongst survivors who were subjected to hyperoxia versus those who were not $(\mathrm{t}=-0.585 ; \mathrm{P}=0.560)$.

Conclusion: Many critically ill patients are ventilated in the ED using $\mathrm{Vt}$ and $\mathrm{FiO}_{2}$ greater than what is recommended. These unadjusted results suggest that the delivery of excessive $\mathrm{FiO}_{2}$ in the ED may negatively impact patient outcomes.

Keywords: Hyperoxia, Lung-protective, Ventilation

\section{MP24}

Antibiotic prescribing and outcomes for patients with purulent skin and soft tissue infections in the emergency department

T. Ibrahim, MSc, C. Thompson, MSc, S. McLeod, MSc, PhD, B. Borgundvaag, $\mathrm{MD}, \mathrm{PhD}$

Schwartz/Reisman Emergency Medicine Institute, Sinai Health, Toronto, ON

Introduction: Skin and soft tissue infections are common presentations in the emergency department (ED). Current guidelines suggest adjuvant antibiotics after incision and drainage (I\&D) of small, uncomplicated skin abscesses may improve treatment outcomes, minimize pain and prevent recurrence. The objective of this study was to determine which factors were associated with antibiotic prescribing at ED discharge, which antibiotics were prescribed, and if antibiotic selection affected patient outcomes.

Methods: This was a retrospective medical record review of adult patients ( $\geq 18$ years) discharged from an academic hospital ED (annual census 65,000) over a 2-year period with diagnosis of an uncomplicated skin abscess. Patients were excluded if they were febrile, immunocompromised, or their infection was related to hospital admission or recent surgery. Using a study-specific data extraction tool, trained research staff recorded clinical signs and symptoms, investigations, ED management, and antibiotic prescribing. Outcomes of interest included any unplanned return to the ED within 30 days, repeat I\&D, and escalation to IV antibiotics.

Results: Of 389 ED visits, 333 (85.6\%) patients underwent I\&D, of which $204(61.3 \%)$ were prescribed antibiotics at discharge. Of the patients who received antibiotics at discharge, 74 (36.3\%) received guideline compliant antibiotics (TMP-SMX or clindamycin). Compared to patients prescribed guideline compliant antibiotics, patients treated with cefalexin were more likely to have an unplanned return ED visit within 30 days $(20.4 \%$ vs $8 \% ; 12.4,95 \%$ CI $1.6-22.6)$, were more likely to have a repeat I\&D (14\% vs $0 \% ; 14,95 \%$ CI $6.5-22.5)$ within 30 days. There was no need for escalation to IV antibiotics in patients who were prescribed guideline compliant antibiotics or those who were prescribed cefalexin.

Conclusion: Antibiotics were prescribed for the majority of uncomplicated abscesses that underwent I\&D. Less than half of patients received antibiotics that were compliant with current guidelines. Compared to those who received cefalexin, patients prescribed guideline compliant antibiotics had fewer unplanned return ED visits and were less likely to have a repeat I\&D within 30 days, consistent with recent literature recommending against cephalosporins in favour of TMP-SMX or clindamycin.

Keywords: Antibiotic, Emergency department, Skin and soft tissue infections

\section{MP25}

Determinants of first trimester spontaneous abortion management in the emergency department: an equity analysis

A. Srajer, M. Wylie, K. Lonergan, BSc, P. Brain, BA, MD, E. Lang, MD
University of Calgary, Calgary, AB

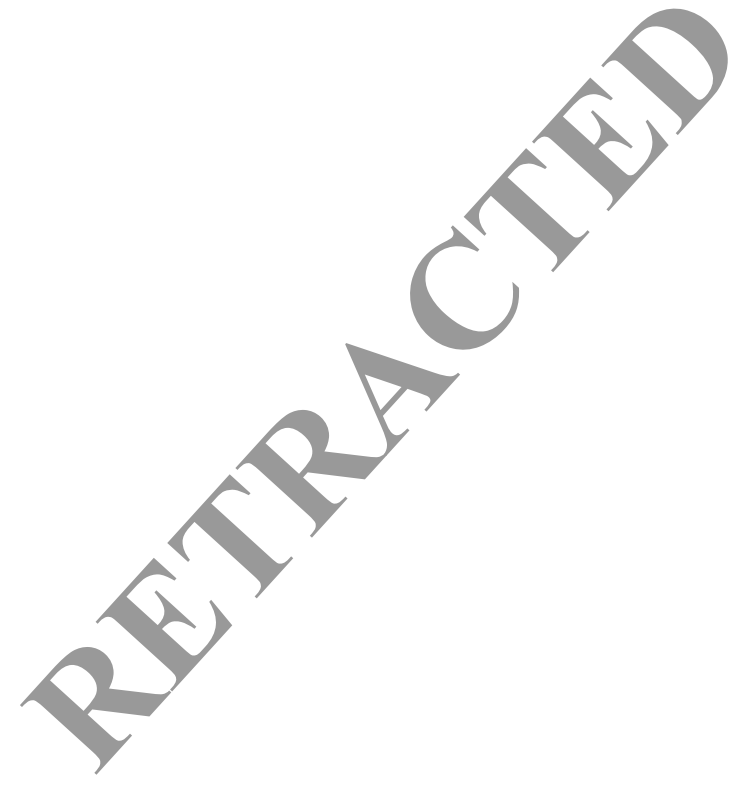

The Authors of Abstract MP25 have retracted this Abstract. Following publication, the authors became aware that the gender data had been erroneously switched. Therefore, the results presented in the abstract are incorrect. All authors agree to this retraction.

\section{MP26}

Evaluating the impact of a pre-arrival CPR quality checklist on resuscitation during a simulated cardiac arrest scenario

D. Drew, MD, M. O’Brien, MD, L. McMurray, MD, A. Krywenky, MD, W. Cheung, MD, MMed, C. Vaillancourt, MD, MSc

University of Ottawa, Department of Emergency Medicine, Ottawa, ON

Introduction: While high quality CPR is recognized as a cornerstone of cardiac arrest management, numerous studies have shown variability in healthcare provider adherence to resuscitation guidelines. We sought to evaluate the effect of a pre-arrival high-quality CPR checklist on emergency resident physician adherence to resuscitation guidelines during a simulated cardiac arrest scenario.

Methods: We conducted a double-blind randomized controlled trial using 1:1 stratified blocks of ten and sealed-opaque envelope allocation concealment. The study took place in a Skills and Simulation Centre. Eligible participants were distributed across post graduate years and enrolled in a Canadian emergency medicine training program (FRCPC or CCFP-EM). The intervention group was presented 
with an iteratively designed, guideline-based CPR quality checklist during the pre-brief and scenario, whereas the control group was not. The simulation scenario consisted of an adult patient arresting shortly after arrival to the emergency department, following which actorprovided ventilations and chest compressions deteriorated in a standardized time and manner. We measured recognition and correction of deteriorating CPR quality and report the proportion of time during which high-quality CPR elements were provided using Mann-Whitney $\mathrm{U}$ testing for comparison of non-parametric data.

Results: Thirty-five of 53 residents completed the study before COVID-19 closure. We observed a lower proportion of total arrest time with no or low quality chest compressions in the intervention group [median $=0.29(25-75$ th interquartile $0.29-0.38)$ ] compared to the control group [median $=0.42(25-75$ th interquartile $0.31-0.49)$ ] $\mathrm{P}=0.07$. When chest compressions were provided, we observed a significantly lower proportion of poor quality chest compressions in the intervention group [median $=0.24(25-75$ th interquartile $0.2-$ $0.38)]$ vs. the control arm [median $=0.42(25-75$ th interquartile 0.33 $0.61)] \quad P=0.03$. Furthermore, we observed a significantly lower proportion of time with unacceptably high bag mask ventilation rates in the intervention group [median $=0.81(25-75$ th interquartile $0.61-$ $1)$ ] vs. the control arm [median $=1(25-75$ th interquartile 1-1)] $\mathrm{P}=0.02$.

Conclusion: Our high-quality CPR checklist improved adherence to resuscitation guidelines amongst resident physicians. This low cost, easily adaptable innovation warrants further study as it may improve cardiac arrest patient outcomes.

Keywords: Checklist, CPR, Resuscitation

\section{MP27}

Pre-defibrillation end-tidal $\mathrm{CO}_{2}$ and defibrillation success in outof-hospital cardiac arrest

J. Kwong, MD, MSc, I. Drennan, PhD, ACP, L. Turner, PhD, S. Cheskes, MD

University of Toronto, Toronto, ON

Introduction: Previous studies have shown that pre-shock $\mathrm{ETCO}_{2}$ may be an indicator of successful termination of ventricular fibrillation $(\mathrm{VF})$ and could guide resuscitation efforts and timing of shocks. However, these studies have been limited to measurement of $\mathrm{ETCO}_{2}$ using advanced airways. No studies have evaluated the relationship between $\mathrm{ETCO}_{2}$ and shock success using a bag-valve mask (BVM) or supraglottic airway (SGA).

Objective: To assess the relationship between pre-shock $\mathrm{ETCO}_{2}$ (measured with BVM, SGA, or an endotracheal tube (ETT)) and VF termination success.

Methods: We reviewed all out-of-hospital cardiac arrests (OHCA) with initial VF rhythm from January 2018 to December 2019 from two urban paramedic services. The ETCO2 level before each shock was compared to the rhythm assessed at $2 \mathrm{~min}$ (first rhythm analysis) after shock delivery. The rhythm was recorded as VF, pulseless electrical activity (PEA), asystole, or return of spontaneous circulation (ROSC). The primary outcome was to determine the association between pre-shock $\mathrm{ETCO}_{2}$ and VF termination. VF termination was defined as absence of refibrillation after shock delivery and $2 \mathrm{~min}$ of CPR. Adjusted multi-level logistic regression was used to account for repeated measurements for each case. Airway type (BVM vs. SGA/ETT) was included as an a priori interaction term with other covariates included in the initial model with backwards elimination.

Results: In total, 271 cases were reviewed (mean age 63, 79\% male) with 904 shocks provided (mean shocks per patient 3.4), and 398 shocks with pre-shock $\mathrm{ETCO}_{2}$ measurements. Mean pre-shock
$\mathrm{ETCO}_{2}$ by airway type were: $\mathrm{BVM}-30.1 \mathrm{mmHg}(\mathrm{n}=291), \mathrm{SGA}-$ $37.0 \mathrm{mmHg}(\mathrm{n}=55)$, ETT-40.3 mmHg $(\mathrm{n}=52)$. BVM had lower $\mathrm{ETCO}_{2}$ values when compared to advanced airway (SGA/ETT) values (Welch $\mathrm{t}$ test, $\mathrm{t}=-2.18, \mathrm{p}=0.04$ ). $\mathrm{ETCO}_{2}$ measurements were divided into tertials $(<26 \mathrm{mmHg} ; 26-36 \mathrm{mmHg} ;>36 \mathrm{mmHg})$. $\mathrm{ETCO}_{2}$ was not associated with VF termination when assessed as tertials [T2 adjOR $1.05(0.47,2.34) \mathrm{p}=0.90, \mathrm{~T} 3$ adjOR $0.72(0.29$, 1.78) $\mathrm{p}=0.48-\mathrm{T} 1$ reference] or as a continuous variable [adjOR 0.99 $(0.97,1.02) p=0.69]$. Airway type was not a significant covariate or effect modifier. Mean pre-shock $\mathrm{ETCO}_{2}$ for shocks leading to the following results were: no VF termination- $32.2 \mathrm{mmHg}$, PEA$32.8 \mathrm{mmHg}, \quad$ asystole $-32.4 \mathrm{mmHg}$, and $\mathrm{ROSC}-32.5 \mathrm{mmHg}$ $($ ANOVA, $\mathrm{p}=0.99$ )

Conclusion: Pre-shock $\mathrm{ETCO}_{2}$ was not associated with shock success, regardless of airway type used. BVM had significantly lower mean $\mathrm{ETCO}_{2}$ values than advanced airways.

Keywords: Cardiac arrest, End-tidal $\mathrm{CO}_{2}$, Ventricular fibrillation

\section{MP28}

Sodium valproate compared to dopamine antagonists for the management of acute episodic migraine headache: a systematic review and meta-analysis

J. Viau, MD, MA, D. Patel, BSc, W. Cheng, PhD, M. Cortel, MD, A. Nath, MD, J. Perry, MD, MSc

Department of Emergency Medicine, University of Ottawa, Ottawa, ON

Introduction: Migraine is the most common primary headache disorder diagnosed in the Emergency Department. The primary objective of this systematic review is to compare the efficacy of sodium valproate to dopamine antagonists in relieving pain due to acute migraine headaches.

Methods: Two research librarians created a search strategy that included Embase, Medline, and Cochrane Database of Clinical Trials from inception to June 1st, 2020. Two investigators individually identified randomized control trials that analyzed adult patients with acute migraine presenting to the emergency department or acute clinical setting which compared sodium valproate to a dopamine antagonist with the aim of relieving pain. Primary outcome was headache relief by $1 \mathrm{~h}$ from treatment. Secondary outcomes included pain relief at 2 and $24 \mathrm{~h}$, relief of associated symptoms (nausea, photo-/phonophobia, dizziness, etc.), and need for rescue analgesia. Meta-analysis was performed and presented as odds ratios. Qualitative data were synthesized separately, and the quality of the studies was assessed with the Cochrane Risk of Bias 2 tool.

Results: Four randomized control trials were identified from an initial pool of 288 titles with a total of 470 patients. Two studies compared sodium valproate to a dopamine antagonist alone and two compared sodium valproate to a dopamine antagonist plus one other agent (sumatriptan or dihydroergotamine). Three studies were included in the meta-analysis and reported a pooled odds ratio of $1.14(95 \% \mathrm{CI}$ $0.35,4.24 ; \mathrm{p}=0.001 ; \mathrm{I}^{2} 86 \%$ ) in favour of sodium valproate for pain relief at $1 \mathrm{~h}, 1.21\left(95 \% \mathrm{CI} 0.29,5.06 ; \mathrm{p}=0.001 ; \mathrm{I}^{2} 86 \%\right)$ at $2 \mathrm{~h}$, and $0.42\left(95 \% \mathrm{CI} 0.25,0.70 ; \mathrm{p}=0.52 ; \mathrm{I}^{2} 0 \%\right)$ at $24 \mathrm{~h}$. The fourth paper reported a median pain reduction of $64.5 \mathrm{~mm}$ and $9 \mathrm{~mm}$ for the dopamine antagonists and sodium valproate, respectively, on the 100point VAS scale at $1 \mathrm{~h}$. Two articles reported improvement of associated migraine symptoms, both favouring the dopamine antagonist group. Three articles reported the need of rescue analgesia (two of which in favour of dopamine antagonist), however pooling is inconclusive and has excessive heterogeneity.

Conclusion: Sodium valproate is not more effective than dopamine antagonists at reducing headache pain at 1 or $2 \mathrm{~h}$ and is inferior to 
dopamine antagonists at $24 \mathrm{~h}$ in the management of patients with acute migraine.

Keywords: Dopamine antagonist, Migraine, Valproate

\section{Poster}

P001

Law enforcement personnel in the emergency department: a scoping review

S. Abolhassani, BSP, K. Durr, BSc, MD, M. Mela, MSc, MBBS, J. Stempien, BSc, MD, E. Watson, MLIS, T. Oyedokun, MBChB, MMed

University of Saskatchewan, Saskatoon, SK

Introduction: The presence of law enforcement personnel (LEP) is a common and inevitable reality in the emergency department (ED). Their role is diverse and benefits patients and healthcare workers alike. However, LEP presence in the ED also carries unavoidable challenges that can further complicate patient care and damage the inter-professional relationship. Our scoping review aimed to further explore this intricate relationship. The primary objective was to describe the interactions between LEP and ED personnel, and the associated impacts on patient care. Our secondary objective was to identify strategies to optimize interactions between LEP and ED personnel that improve patient care.

Methods: A literature search was conducted using MEDLINE, EMBASE, and PsycINFO to identify relevant publications. These databases were searched from inception until April 2020. Subject headings and Keywords related to the concepts of LEP and the ED were searched by an experienced librarian in collaboration with our research team. Results were limited to English language and publication within the past 25 years (1996-2020). A total of 3447 articles were identified for review, with 47 included for data abstraction. Two investigators independently screened titles and abstracts, reviewed full text articles, and extracted data. Discrepancies were resolved by a third investigator. A standard data extraction form was pilot-tested prior to data extraction. Data was categorized under LEP motive for ED presence, patient confidentiality/privacy, LEP length of stay, healthcare team dynamics, quality of care, and optimization strategies.

Results: Common themes requiring LEP presence in the ED included security, trauma-related patient transport, Mental Health Act patient transport, and most commonly, information gathering. Patient confidentiality/privacy and LEP length of stay were negatively affected by LEP presence in the ED. Healthcare team dynamics and quality of care were positively and negatively affected by the presence of LEP in the ED. Strategies described to optimize interactions between LEP and ED personnel, as well as those aimed at improving patient care varied significantly based on region.

Conclusion: Patient confidentiality/privacy is the key issue identified when encountering LEP in the ED. Strategies aimed at improving collaboration between LEP and ED staff, while ensuring patient confidentiality, are essential in order to foster a positive inter-professional relationship while maintaining proper patient care.

Keywords: Emergency department, Law enforcement personnel, Police

P002

Kids save lives: the reality of CPR and AED training in Ontario schools
K. Allan, BSc, MSc, PhD, T. Jefkins, BSc, E. O’Neil, BA, P. Dorian, MD, MSc, S. Lin, MD, MSc

St. Michael's Hospital, Toronto, ON

Introduction: To increase rates of bystander cardiopulmonary resuscitation (CPR), the Ontario government mandated CPR and automated external defibrillator (AED) training as part of the Grade 9 Physical Health and Education curriculum in the 1990s. Although adherence to the curriculum is mandatory, how each school implements the training is up to each individual school board.

Objectives: The primary objective of this study was to survey all types of publicly funded schools to understand the scope of CPR and AED training to both staff and students, including barriers and facilitators to implementing training.

Methods: We created a semi-quantitative online survey using the SurveyMonkey ${ }^{\circledR}$ tool consisting of 23 questions, both closed and open ended, aimed at Principals and Vice-Principals. We recruited via social media platforms, snowball sampling and professional administrator associations.

Results: From Jan to Feb 2020, we recruited participants from 205 schools, of which 120 (58.5\%) were elementary, 25 (12.2\%) were middle and 60 (29.3\%) were high schools. Collectively, these schools and school boards represent $4.24 \%(205 / 4828)$ and $20.8 \%(15 / 72)$, respectively, of all schools and school boards in Ontario. Most schools $(70 \%, 144 / 205)$ had an AED installed and almost 60\% (119/ 205) required staff to have CPR training. Only $18.8 \%$ (34/181) of schools reported training students, with high schools reporting higher rates of training compared to elementary and middle $(56.3 \%$ high school vs. $4.0 \%$ middle vs. $5.6 \%$ elementary, $\mathrm{p}<0.0001)$. Major barriers to providing training included lack of funding, time, trainers, and awareness of the mandate.

Conclusion: Most of the surveyed public schools in Ontario have an AED installed and provide CPR training to staff. Despite government mandated training for high school students, only 56\% of surveyed schools offer it. More research is needed to understand the barriers to implementing this lifesaving training in schools.

Keywords: Barriers and facilitators, CPR/AED training, Schools

\section{P003}

Measuring heat illness with an administrative coding algorithm: test characteristics and findings

H. Baassiri, BSc, T. Varghese, BSc, K. Clemens, MD, MSc, B. Vujcic, BSc, J. Yan, MD, MSc

Western University, London, ON

Introduction: Climate change is having an increasingly significant and severe impact on human health and has been associated with acute heat illness (AHI). The objective of this study was to assess the validity of an administrative coding algorithm of International Classification of Disease 10th revision (ICD-10) codes for AHI in Canadian emergency departments (EDs).

Methods: Two independent reviewers retrospectively abstracted medical records from two EDs in Ontario between May and September 2013-2018. Sample size calculation demonstrated a need to manually review 62 charts coded as having an ICD-10 code for heat illness and 964 randomly selected charts. We determined the positive predictive value (PPV), sensitivity $(\mathrm{Sn})$, specificity $(\mathrm{Sp})$ and negative predictive value (NPV) of our coding algorithm using a Gold standard definition of an AHI; a chart-documented heat exposure with a heat-related complaint (e.g. syncope while working outdoors on a hot day). In both abstractions, $20 \%$ of cases underwent a blinded duplicate review. 
Results: A total of 326,702 ED visits were included over the study period with 208 charts having an ICD-10 code for heat illness. Average patient age of Gold Standard AHI vs non-heat illness ED presentations was 37 and 42 years of age, respectively. 53\% and 49\% of patients were male, respectively. $4.8 \%$ and $17.4 \%$ were admitted, while $16.7 \%$ and $6.6 \%$ of patients left against medical advice, respectively. No significant differences were seen in triage vital signs or hematological investigations. $88 \%$ of all heat illness presentations presented following a period of physical exertion. In our review of 964 random records, 4 cases were identified as AHI, 3 of which did not have an appropriate ICD-10 code, while 960 were appropriately labelled as not AHIs. In our review of 62 previously coded heat illness, 38 were found to be appropriately coded and 24 inappropriately coded as AHI ICD-10 (Cohen's unweighted $\mathrm{k}=0.9 \pm 0.1$ ). ICD-10 codes used to code AHIs in EDs have a Sn of $25 \pm 42.4 \%$, Sp of $100 \pm 0 \%$, PPV of $61.3 \pm 12.1 \%$ and NPV of $99.7 \pm 0.4 \%$. Conclusion: Based on our results, ICD-10 codes may not accurately measure the burden of heat illness in Canada. However, our results suggest that these codes may provide utility in investigating cohorts of patients with potential heat illnesses, for Gold Standard AHI. Our findings suggest that vital signs and laboratory investigations are not adequate parameters to independently investigate for AHI.

Keywords: Coding, Heat, Validation

\section{P004}

What is the prehospital experience of patients presenting to the ED with police with agitation and violent behaviour? A preplanned secondary analysis of the RACKED trial

D. Barbic, MD, MSc, H. Philips, BSc, M. Whyte, G. Sidhu, F. Besserer, MD, G. Andolfatto, MD, B. Grunau, MD, MSc, F. Scheuermeyer, MD, MSc, W. MacEwan, MD, H. Wong, PhD, W. Honer, MD, S. Barbic, PhD

\section{UBC, Vancouver, BC}

Introduction: Canadian evidence is limited concerning the prehospital experience of patients presenting to the ED accompanied by police, and displaying agitation and violent behaviour. The objective of this study was to describe the use of prehospital force (PHF) by police in patients presenting to the ED with agitation and violent behaviour.

Methods: This was a pre-planned secondary analysis of the Rapid Agitation with Ketamine in the Emergency Department (RACKED) trial, the methods of which have been reported separately. For this analysis, we describe the prehospital experience of patients presenting to the ED with agitated and violent behaviour. The level of agitation was described using the Richmond Agitation and Sedation Scale (RASS). We used counts, means, medians and 95\% confidence intervals. Chi-square and Fisher exact tests were used to assess differences between the study groups.

Results: Eighty patients were enrolled in the RACKED trial; prehospital data was available for $75(94 \%)$. Fifty-two (69\%) were male, and median age was 35.0 (IQR 29.0-42.0). Sixty-two patients $(86 \%)$ had a history of substance use, $18(25 \%)$ had a prior history of schizophrenia, and $16(22 \%)$ a prior history of bipolar disorder. The police used PHF in 54 patients (72\%) for the purposes of apprehension and transport to hospital. Handcuffs $(n=47,63 \%)$, other physical restraints $(n=15,20 \%)$, and electrical taser use $(n=2,3 \%)$ were the most common methods use by police. None received chemical restraints (pepper spray). The median duration of physical restraint use was 30 min (IQR 20-50). Thirty three patients $(63 \%)$ in the PHF group had a RASS score of $4+$, eight patients (38\%) in the non-PHF group had a RASS score of $4+(\mathrm{p}=0.06)$. The PHF group had 18 patients $(33 \%)<29$ years, compared to 2 patients $(9.5 \%)$ in the non-PHF group $<29$ years $(\mathrm{p}=0.08)$. Hospital admission occurred for 22 patients (41\%) in the PHF group, and 5 patients (17\%) in the non-PHF group $(\mathrm{p}=0.17)$.

Conclusion: Physical force and restraints are commonly used by police for the apprehension and transport of persons with agitation and violent behaviour to the ED. Opportunities for improved coordination of care with EHS, police and emergency departments (ED) likely exist, and further research is warranted to improve the prehospital care of this marginalized patient population.

Keywords: Force, Ketamine, Prehospital

\section{P005}

What proportion of patients presenting to the ED with substance induced psychosis are admitted to hospital?

D. Barbic, MD, MSc, M. Whyte, G. Sidhu, A. Luongo, A. Chakraborty, PhD, MBBS, S. Barbic, PhD, F. Scheuermeyer, MD, $\mathrm{MSc}, \mathrm{W}$. Honer, MD

UBC, Vancouver, BC

Introduction: Substance induced psychosis (SIP) is a common problem encountered in Canadian emergency departments (EDs). Little evidence exists about the ED stay, hospital admissions or outcomes of these patients.

Methods: We conducted a multi-centre retrospective medical records review. Consecutive patients were identified by discharge ED diagnosis using the local ED database, and data was abstracted from individual patient records by 3 trained research assistants. We used a standardized case definition of: (1) amphetamine intoxication or use plus one of: "disorganized thought", "bizarre behaviour", or "delusional behaviour"; or (2) "psychosis NOS" plus a positive urine drug screen (UDS) and one of the following: police arrival, mental health certification, physical or chemical restraint use. Continuous variables were described using counts, means, medians and IQR. The primary outcome was proportion of patients admitted to hospital; associated variables were explored with logistic regression.

Results: Between Jan 2, 2015 and Jan 1, 2019, we identified 520 patients with median age $34($ IQR 27-44) and 71\% $(\mathrm{n}=370)$ male. Mean ED LOS was $13.5 \mathrm{~h}$ (95\% CI 11.3-17.7). Most patients $(\mathrm{n}=305,58.7 \%)$ endorsed using amphetamines, crack cocaine $(\mathrm{n}=68,13.1 \%)$, and fentanyl $(\mathrm{n}=53,10.2 \%)$. The most common comorbidities were schizophrenia $(23.7 \%)$ and hepatitis C $(21.2 \%)$. Physical restraints were required by $27.3 \%$ of patients, with a median duration of $100 \mathrm{~min}$ (IQR 50-135). Two hundred thirty seven patients $(46.1 \%)$ were admitted to hospital, with median hospital LOS $=1$ day (IQR 0-6). Logistic regression controlling for age, use of fentanyl, and prior history of schizophrenia, demonstrated that amphetamine use (self-report or UDS +) was associated with an increased likelihood of hospital admission [OR 3.9 (95\% CI 1.7-8.8)].

Conclusion: Almost half of patients presenting to the ED with SIP are admitted to hospital. Amphetamine-induced psychotic disorder is associated with an increased likelihood of hospital admission.

Keywords: Substance psychosis

\section{P006}

What are the ED patterns of use for patients presenting to the ED with substance induced psychosis?

D. Barbic, MD, MSc, M. Whyte, G. Sidhu, A. Luongo, A. Chakraborty, PhD, MBBS, S. Barbic, PhD, F. Scheuermeyer, MD, MSc, W. Honer, MD

UBC, Vancouver, BC 
Introduction: Substance induced psychosis (SIP) is a common problem encountered in Canadian emergency departments (EDs). Little evidence exists about the ED stay or outcomes of these patients. Methods: We conducted a multi-centre retrospective medical records review. Consecutive patients were identified by discharge ED diagnosis using the local ED database, and data was abstracted from individual patient records by 3 trained research assistants. We used a standardized case definition of: (1) amphetamine intoxication or use plus one of: "disorganized thought", "bizarre behaviour", or "delusional behaviour"; or (2) "psychosis NOS" plus a positive urine drug screen and one of the following: police arrival, mental health certification, physical or chemical restraint use. Positive imaging findings were defined as those patients with radiologist reports of clinically significant abnormalities. Continuous variables were described using counts, means, medians and IQR. The primary outcome was the number of return ED visits (after the index SIP visit), associated variables were explored with negative binomial regression.

Results: Between Jan 2, 2015 and Jan 1, 2019, we identified 520 patients with median age $34($ IQR $27-44)$ and $71 \%(\mathrm{n}=370)$ male. Mean ED LOS was 13.5 h (95\% CI 11.3-17.7). X-rays were completed for 46 patients $(8.8 \%)$ [19 positive findings (41.3\%)], included pneumonia, COPD, ulna and calcaneus fractures. Forty-one patients (7.9\%) had CT imaging [11 positives $(26.8 \%)$ ], including septic arthritis and lacunar infarcts. Three had US studies 2 positive findings $(66 \%)$ ] of hepatic cirrhosis, and $3(0.6 \%)$ had MRI (0 positive findings). Non-psychiatric medical services were involved for 124 patients $(23.8 \%)$ in the ED [18.3\% $(\mathrm{n}=95)$ addiction medicine; $3.3 \%$ $(\mathrm{n}=17)$ internal medicine] Almost half of all patients $(\mathrm{n}=230$, $44.2 \%$ ) presented to the ED 30 days before their index visit for SIP, median 2 visits (IQR 1-3). Including those admitted to hospital, 90 patients (17.3\%) returned to the ED within 30 days of their index SIP visit, median 2 (IQR 1-3). Negative binomial regression controlling for age, amphetamine or fentanyl use, and prior Hx of schizophrenia, demonstrated no clear predictor of increased return ED visits.

Conclusion: Patients presenting to the ED with SIP have often visited the ED in the 30 days before the index, were frequently found to have positive findings on diagnostic imaging, and returned to the ED a median of twice within 30 days of their index visit.

Keywords: Substance psychosis

\section{P007}

What is the $\mathbf{3 0}$ day mortality of patients presenting to the ED with substance induced psychosis?

D. Barbic, MD, M. Whyte, A. Luongo, G. Sidhu, A. Chakraborty, PhD, MBBS, S. Barbic, PhD, F. Scheuermeyer, MD, MSc, W. Honer, MD

\section{UBC, Vancouver, BC}

Introduction: Substance induced psychosis (SIP) is a common problem encountered in Canadian emergency departments (EDs). Little evidence exists about the ED stay or outcomes of these patients. Methods: We conducted a multi-centre retrospective medical records review. Consecutive patients were identified by discharge ED diagnosis using the local ED database, and data was abstracted from individual patient records by 3 trained research assistants. We used a standardized case definition of: (1) amphetamine intoxication or use plus one of: "disorganized thought", "bizarre behaviour", or "delusional behaviour"; or (2) "psychosis NOS" plus a positive urine drug screen and one of the following: police arrival, mental health certification, physical or chemical restraint use. Continuous variables were described using counts, means, medians and IQR. The primary outcome was the 30 day mortality (identified with linkage with vital statistics), associated variables were explored with logistic regression.
Results: Between Jan 2, 2015 and Jan 1, 2019, we identified 520 patients with median age $34($ IQR 27-44) and 71\% $(\mathrm{n}=370)$ male. Mean ED LOS was 13.5 h (95\% CI 11.3-17.7). Most patients $(\mathrm{n}=305,58.7 \%)$ endorsed using amphetamines, crack cocaine $(\mathrm{n}=68,13.1 \%)$, and fentanyl $(\mathrm{n}=53,10.2 \%)$. The most common comorbidities were schizophrenia $(23.7 \%)$ and hepatitis C $(21.2 \%)$. Physical restraints were required by $27.3 \%$ of patients, with a median duration of $100 \mathrm{~min}$ (IQR 50-135). At the index visit for SIP, 2 patients $(0.4 \%)$ were admitted to the ICU (pt. $1=$ hypoxic respiratory failure; pt. 2 = upper GI bleed). One percent of patients $(n=5)$ had died at 30 days after the index SIP ED visit. Logistic regression controlling for age, amphetamine use and ICU admission revealed that fentanyl use (self report or UDS + at index visit) was associated with an increased likelihood of death at 30 days [OR 21.4 (95\% CI 2.7-168.0)].

Conclusion: One percent of patients presenting to the ED with SIP were not alive at 30 days after their index visit. Use of fentanyl in this patient population was associated with a markedly increased likelihood of death at 30 days.

Keywords: substance psychosis.

\section{P008}

The northern amazing and awesome model of hospital-wide QI rounds: a qualitative analysis

M. Bhatia, BSc, MD, R. Ohle, MBChB, MD, MSc, C. Cook, PhD, S. McIsaac, MBChB, MEd, A. Shanmugarajah, MD

\section{Health Sciences North, Sudbury, ON}

Introduction: The Northern Amazing and Awesome (A\&A) model has been proposed as a structured approach to quality improvement rounds grounded in positive deviance. Its goal is to identify and amplify behaviours and processes that lead to exceptional patient outcomes. The objective of this study was to assess the learning impact after local implementation of this model.

Methods: 226 attendees (physicians, surgeons, nurses, respiratory therapists, administrative staff) were surveyed at 3 A\&A rounds over a 1-year period at a tertiary care institution. Inductive thematic analysis was conducted on free-text responses to three questions (How did this session contribute to meeting your personal learning goals? What "pearl" will you take back to your practice? Do you have any other comments?). The data was analyzed by two researchers in five phases: (1) The data was categorised by question type; (2) Initial inductive codes were generated in a systematic fashion; (3) Inductive codes were collated into potential subthemes and themes; (4) A thematic map was generated and reviewed; (5) overarching themes were defined and named.

Results: The responses of 63/226 attendees were obtained. Responses were invariably positive. Inductive thematic analysis of the responses identified four major themes: an improved understanding of positive deviance and the role of recognizing local clinical successes; appreciation of effective communication techniques; increased medical knowledge specific to presented cases; new awareness of local resources and systemic processes.

Conclusion: This analysis provides an understanding of the potential learning impact that was consistent across multiple rounds. The analysis demonstrated a diverse range of learning outcomes from clinical content to techniques for positive interdisciplinary work culture. This consistency of themes suggests that the learning impact could be attributable to A\&A model, rather than a specific session. Comments suggest that participants would value increased delivery and frequency of A \& A rounds. These rounds can be readily applied to any hospital seeking to enhance quality of care, work culture, and patient safety. 
Keywords: Awesome and amazing rounds, Grand rounds, Positive deviance

\section{P009}

Educational innovation: video in situ simulation for medical student education during the COVID-19 pandemic

M. Bilic, BSc, A. Nagji, BHSc, MD, E. Hanel, MSc, MB, BAO

McMaster University, Hamilton, ON

Innovation concept: The COVID-19 pandemic has placed several constraints on medical education, limiting students' in-person clinical experiences, especially in situations involving aerosol-generating medical procedures. Given this limitation, we designed a novel curriculum in the form of video in situ simulation to expose medical students to COVID-19 protected intubations.

Methods: Using videoconferencing software, students in small groups were paired with faculty and resident facilitators. Together, they watched a video of an in situ simulation of a protected intubation for respiratory failure secondary to COVID-19.

Curriculum, tool, or material: The 9-min video was a recording of an unrehearsed in situ simulation at a busy emergency department in Hamilton, Ontario. The video contained six integrated discussion points appearing on the screen at discrete times, during which the video was paused for discussion. Topics covered through the discussion points included: aerosol-generating medical procedures and protected intubation principles, closed loop communication, use of negative pressure rooms, and donning and doffing of PPE. Feedback for the innovation was collected via the administration of surveys prior to and following the session. The goal of feedback collection was to gauge students' perspectives on psychological safety during the session and to evaluate the session's utility in increasing student comfort with protected intubations. 13 paired pre- and post-session surveys responses were analyzed. $92 \%$ of survey respondents $(12 / 13)$ either strongly agreed or agreed that the learning atmosphere was comfortable, and $62 \%$ (8/13) either strongly agreed or agreed that it was easy to get actively involved in the session. When comparing the pre-session and post-session surveys, $84 \%$ of respondents (11/13) reported an increased level of comfort with intubation for respiratory failure, and $92 \%$ of respondents (12/13) reported an increased level of comfort with protected intubation following the session.

Conclusion: In conclusion, this simple innovation provided a comfortable learning environment that drives engagement and discussion, while allowing for flexibility and ensuring learner safety. This session was integrated into the emergency medicine core clerkship at McMaster University, substituting in-person simulation sessions that were no longer feasible given physical distancing requirements. Future steps include adapting to various scenarios and levels of training.

Keywords: Innovations in EM education, Simulation

\section{P010}

Cardiac monitoring of hospitalized patients in the emergency department

R. Bradley, MD, V. Thiruganasambandamoorthy, MSc, MBBS, E. Kwok, MD, MSc, MHA

Department of Emergency Medicine, University of Ottawa, Ottawa, ON

Introduction: Emergency department (ED) crowding is a growing crisis and beds with cardiac monitors are a very limited resource. This study aims to identify risk factors for serious adverse events (SAE) in order to optimize the use of monitors. Secondary objectives were to assess the proportion of patients who received interventions due to monitoring and total time spent on cardiac monitors.

Methods: We performed a 2-week health records review of hospitalized patients in the ED on cardiac monitors. We collected baseline characteristics and outcomes including serious adverse events (SAE; e.g., arrhythmia, hypotension) during the ED stay. We used descriptive statistics and a logistic regression analysis of 6 pre-determined variables to identify risk factors for SAE requiring intervention: age, past medical illness (chronic: hypertension, diabetes, cancer, COPD, heart failure; or severe: pacemaker, previous ICU stay, previous cardiac arrest, CAD, arrhythmia), abnormal vital signs $(\mathrm{HR}<50$ or $>105 \mathrm{bpm}, \mathrm{RR}<8$ or $>25, \mathrm{SpO} 2<90 \%$ or supplemental $\mathrm{O} 2$, mean arterial pressure $<60$ or $>160 \mathrm{mmHg}, \mathrm{T}<35$ or $>38{ }^{\circ} \mathrm{C}$ ), admission diagnosis, admission destination (monitored or unmonitored location), CTAS score.

Results: 305 patients (mean age 63.7 years; $52.5 \%$ male) of whom 56 $(18.4 \%, 9$ with arrhythmia) suffered SAE. Patients under internal medicine and cardiology utilized the most hours on cardiac monitors (1716.0, 917.6 $\mathrm{h}$ respectively) and had the highest numbers of patients with SAE requiring intervention (33 patients total). Patients under neurology and medical oncology had the highest SAE rate (38.5\% and $35.7 \%$, respectively). Predictors of SAE requiring intervention were: chronic (OR 4.89, $\mathrm{p}=0.04$ ) or severe (OR 4.04, $\mathrm{p}=0.04)$ illness, abnormal vital signs $(\mathrm{OR} 3.55, \mathrm{p}=0.002)$ and monitored destination (OR 2.32, $\mathrm{p}=0.03)$. These risk factors predicted SAE during ED stay with $100 \%$ (93.2-100\%) sensitivity and $4.7 \%(2.5-8.1 \%)$ specificity.

Conclusion: Our study identified risk factors for identification of a SAE requiring intervention among hospitalized patients who undergo cardiac monitoring during their ED stay. Patients with no chronic or severe illness, normal vital signs and destined to an unmonitored inpatient location can potentially be removed from cardiac monitoring in the ED. Prospective studies are needed to develop a safe clinical decision tool for discontinuation of cardiac monitoring in the ED for hospitalized patients.

Keywords: Emergency department cardiac monitors, Emergency department crowding

\section{P011}

Diagnostic yield of head computerized tomography in elderly emergency department patients with possible delirium: a retrospective chart review

J. Butcher, MD, M. Smith, BSc, L. Roberts, BSc, MD, MSc, B. Ellis, $\mathrm{MD}, \mathrm{MSc}$

University of Saskatchewan, Saskatoon, SK

Introduction: Delirium has a broad differential diagnosis and often suggests grave pathology in the elderly; therefore, prompt determination of the underlying cause is essential for appropriate management of these patients. Computed tomography of the head (CT-Head) may have a role in determining the cause of delirium in certain circumstances, however inpatient studies have demonstrated that CT-head is an overused imaging tool in this population. There is currently a paucity of emergency department (ED) based research surrounding the use of CT-Head in delirium. This study aims to describe the diagnostic utility of CT-Head in elderly patients presenting to the ED with delirium.

Methods: We conducted a retrospective chart review of patients 65 years and older who visited Saskatoon-area EDs with suspicion of delirium from 2016 to 2020 . We compared charts of patients who received CT-Head and those who did not to examine for differences 
between groups. Our primary outcome was to note the proportion of patients who received CT-Head who had a management changing finding. Secondary objectives were to compare proportions of patients who did and did not receive CT-Head in terms of their presenting symptoms and their disposition from the ED, and to describe the indications and findings of performed CT-Head scans. Chi-squared tests were used to compare for differences between groups where appropriate.

Results: We examined a total of 630 patient charts. Of these, 138 charts were excluded as the patient either presented to the ED for direct care or had reference to a delirium irrelevant to their current ED visit, leaving 492 included charts. We found that of those who received CT-Head $(n=279)$ only $13(4.7 \%)$ had management changing findings. Of those with findings on CT-Head, the indications listed for ordering CT-Head included a new focal neurological deficit $(n=2)$, trauma $(n=3)$, acute confusion $(n=7)$, altered level of consciousness or obtundation $(\mathrm{n}=2)$. Further, delirious patients who did not receive CT-Head $(n=213)$ were more likely to be discharged $(p<0.01)$ and have an absence of new focal neurological deficits $(\mathrm{p}<0.01)$.

Conclusion: These results show CT-Head is ordered in over half of patients presenting to the ED with symptoms of delirium, though positive findings are rare and occur most commonly in patients with other symptoms suggestive of intercranial abnormalities. This suggests that CT-Head may be overused in this patient population in the ED.

Keywords: Computerized tomography, Delirium, Geriatrics

\section{P012}

\section{A pilot quality improvement project implementing a nursing} directive for children with suspected appendicitis

H. Chaudhari, BHSc, R. Rebinsky, BSc, M. Schneeweiss, BSc, E. Rullo, BSc, MBA, N. Stein, MD, A. Kam, MD, MPH, G. Thompson, MD, A. Worster, MD, MSc, PhD, M. Eltorki, MBChB

McMaster University, Hamilton, ON

Background: Although appendicitis accounts for an estimated 7$10 \%$ of abdominal pain cases in the emergency department (ED), patients with suspected appendicitis tend to wait to be seen by a physician, resulting in diagnostic delays. The utility of a nursing directive to expedite the diagnostic process is unclear and needs further exploration. Our study aims to describe key components of suspected appendicitis patients seen at a pediatric ED and pilot a directive that allows nurses to order blood work, urine tests, analgesics, fluids, and ultrasound (U/S) prior to physician assessment. Our aim is to reduce the time from ED presentation to U/S completion by $20 \%$ over 1 year.

Aim statement: A chart review was conducted of children aged 317 years who presented with $\leq 4$ days of acute abdominal pain, right lower quadrant tenderness with walking, and right iliac fossa tenderness between April and October 2019. We excluded patients with chronic medical conditions or neurodevelopmental disorders. Patient baseline characteristics and ED flow metrics were abstracted using a standardized case report form and entered in REDCap. Each chart was reviewed by two reviewers independently. We compared ED flow metrics before and after directive implementation. For differences in proportions, we conducted a $\chi^{2}$ test and for continuous variables, we used Student's t test.

Measures and design: We screened 2400 patient charts and 220 (9.2\%) patients were eligible. Among those, $66(30 \%)$ children had appendicitis. The overall mean age was 11.5 years [95\% CI (11.0, 12.0)] and $140(64 \%)$ were females. The mean duration of time in hours from ED presentation to: (1) blood work, 3.1 [95\% CI (2.6,
3.5)], (2) U/S order, 2.5 [95\% CI $(2.3,2.7)]$, and (3) U/S report, 5.0 [95\% CI $(4.6,5.4)]$. We piloted the directive on 39 patients and achieved a shorter duration of time from ED presentation to blood work by $2.1 \mathrm{~h}[95 \% \mathrm{CI}(1.2,2.9)]$, U/S order by $1.2 \mathrm{~h}$ [95\% CI $(0.8$, $1.7)]$, and U/S report time by $1.3 \mathrm{~h}$ [95\% CI $(0.5-2.1)]$ when compared to standard of care.

Evaluation/results: In our centre, children with suspected appendicitis spend almost $5 \mathrm{~h}$ in the ED prior to U/S results. This pilot quality improvement project demonstrates how a nursing directive can shorten this time by $20 \%$. We will measure the effects of the directive on ED flow metrics and patient experience over 1 year. Keywords: Appendicitis, Directive, Quality improvement and patient safety

\section{P013}

Exploring goals of care discussions in the emergency department: a qualitative study using the theoretical domains framework

L. Cheskes, MD, MSc, A. Patey, PhD, J. Frank, MD, MA(Ed), W. Cheung, MD, MMed

Department of Emergency Medicine, University of Ottawa, Ottawa, ON

Introduction: Goals of care (GOC) discussions involve communication between a patient and the medical team to outline a patient's plan of care that is in keeping with their wishes. While these conversations are essential to determine appropriate care pathways, they often do not occur in the emergency department (ED). We applied the Theoretical Domains Framework (TDF) to explore current practices with regards to GOC discussions in the ED and to identify perceived barriers and enablers to emergency physicians' engagement in these discussions.

Methods: Semi-structured interviews of staff emergency physicians from academic and community hospitals in Ottawa, Canada were conducted. An interview guide based on the TDF was designed to identify factors that may influence GOC discussions in the ED. Interview transcripts were coded using direct content analysis and belief statements were generated. Relevant domains were identified based on presence of conflicting beliefs, frequencies of beliefs reported and perceived influence on GOC discussions in the ED.

Results: In total, 12 emergency physicians were interviewed. Eight of the fourteen TDF domains were identified as influencing emergency physician engagement in GOC discussions in the ED. Participants identified benefits to the patient, physician and healthcare system as enablers to engagement in GOC. However, lack of evidence-based guidelines and a framework to guide the conversation were identified as key barriers. Additionally, environmental factors, such as appropriate space to have these conversations, time pressures and frequent interruptions, were identified as factors impeding GOC discussions in the ED. The emergent nature of patient presentations, patients with limited knowledge of their medical condition, language barriers and social dynamics amongst family members were also perceived to contribute significant challenges. Despite these barriers, there was overwhelming support for future strategies aimed to increase engagement in GOC discussions in the ED, including development of evidence-based approaches to these conversations, inclusion of GOC training in residency education, and use of patient-specific decision aids to clarify personal values.

Conclusion: We employed a systematic qualitative approach to identify key barriers and enablers to GOC discussions in the ED. These factors offer potential targets for behaviour change interventions aimed at enhancing current GOC practices in the ED setting.

Keywords: Theoretical domains framework, Advanced care planning, Goals of care discussions 


\section{P014}

Examining local practice patterns of urinary screening in elderly patients presenting to the emergency department without lower urinary symptoms or sepsis

J. Choi, MD, MPH, C. Tsoutsoulas, MD

University Health Network, Toronto, ON

Introduction: Despite guidelines recommending against routine screening and treatment of asymptomatic bacteriuria (ASB), it continues to be commonly performed for elderly patients presenting to the emergency department (ED). We examined practices among emergency physicians (EPs) regarding the use of urine studies in the workup of elderly patients presenting to the ED with non-specific symptoms.

Methods: We performed a chart review and a survey of EPs. The chart review was conducted at 2 academic EDs and included patients $>65$ years old presenting with chief complaints of weakness, falls, or confusion between March and July 2019. We excluded patients with any systemic inflammatory response syndrome (SIRS) criteria, had focal neurological deficits, or had a Glasgow Coma Scale Score $<14$ to include only patients without signs of infection and who were able to provide a reliable history. The rate of documentation of urinary symptoms and the rate of urine studies performed were analyzed. The survey contained 5 cases based on clinical scenarios from the 2019 guidelines on ASB from the Infectious Diseases Society of America. As per guidelines, these cases would not warrant screening for, nor treatment of, ASB. We asked EPs if they would have ordered a urinalysis, and if they would have prescribed antibiotics and/or sent a culture if one was sent prior to assessment and had a positive result.

Results: The chart review included 31 patients that met our inclusion criteria. $74 \%$ of these patients had a urinalysis performed. Of those patients, $52 \%$ had a subsequent urine culture. Only $35 \%$ of EPs documented whether lower urinary symptoms were present, and only $18 \%$ of those patients endorsed having symptoms. The survey had 46 EP respondents (53\% response rate). A urinalysis was ordered by $26-$ $84 \%$ of EPs. Of those who would not have ordered a urinalysis, 32 $50 \%$ would have acted on a positive result by sending a urine culture, and $9-18 \%$ would have prescribed antibiotics.

Conclusion: Many elderly patients presenting to the ED have urine studies as part of their diagnostic workup despite not having urinary symptoms or signs of infection. Furthermore, our survey suggests that a positive result may sway a clinician to order more downstream tests and treatment even if their pre-test suspicion of a urinary tract infection was low. More research is needed to explore the rationale for this behaviour, which may lead to targeted quality improvement initiatives to reduce this practice.

Keywords: Asymptomatic bacteriuria, Clinical decision making, Practice variation

\section{P015}

A live avatar approach to virtual procedural teaching: an experience from the Toronto Addis Ababa Academic Collaboration in emergency medicine

S. Chun, MD, T. Beyene, MD, J. Bryan, MD, MPH, MA, A. Aspler, MD, MSc, DTM\&H

University of Toronto, Toronto, ON

Innovation concept: Simulation training for procedures is well established in emergency medicine (EM). Interest in applying these techniques virtually is growing due to the COVID-19 pandemic. We introduced a novel approach to virtual procedural education for EM residents at Addis Ababa University (AAU) through the Toronto Addis Ababa Academic Collaboration in Emergency Medicine (TAAAC-EM).

Methods: EM residents from all years participated. From staff and learner feedback, lumbar punctures (LPs) were identified as a focus for procedure teaching. LPs are a routine daily procedure for AAU EM residents and are often performed without direct supervision. Our aim was to emphasize two techniques which would improve overall procedure success, use of: (1) local anesthetic and (2) ultrasound (US) landmarking. Despite availability of lidocaine in this setting, it is not consistently used for LPs at AAU.

Curriculum, tool, or material: Pre-reading materials were sent to learners prior to an online live avatar procedural teaching session. The virtual session consisted of three sections, (1) didactic review, (2) synchronous online LP demonstration, and (3) structured interactive component in which residents guided a live avatar on an LP simulator. Participants were asked to guide a medical student virtually on lumbar puncture steps by posting in an online chatbox sequentially (e.g. prep the skin, draw up 5 cc's of lidocaine). We supplemented this with scenarios where they were required to guide the avatar through troubleshooting required while performing LPs (patient repositioning, inadequate anesthesia, adjusting the level of the LP, relandmarking). Program evaluation by residents indicated they would immediately be able to apply this teaching session to their clinical practice. They reported increased confidence in performing LPs using local anesthetic and US guidance.

Conclusion: We describe a novel synchronous virtual procedure education intervention developed through a global health partnership in the context of the COVID-19 pandemic. Residents developed tools that could improve their ability to complete LPs with greater success. Their baseline comfort with use of local anesthetic and use of US for other procedural indications, lead to an increased likelihood of translational competency. Use of a live avatar for simulation of procedural teaching is a curriculum tool which we believe others could employ while transitioning global health partnerships to virtual education formats.

Keywords: Global health partnerships, Innovations in EM education, Virtual education

\section{P016}

Emergency department presentation changes due to the COVID19 pandemic in Nova Scotia, Canada

T. Dahn, BSc, MD, MSc, P. Atkinson, MBChB, BAO, MA, V. Cole, BSc, D. Dutton, PhD, T. Liu, MA, H. Wiemer, MD, P. Fok, MD, PhD

Dalhousie University, Halifax, NS

Introduction: Multiple reports have indicated a reduction in Emergency Department (ED) patient volumes during the Covid-19 pandemic. Much of the data has looked at all ED presentations and there is a lack of detailed data examining the impact of Covid-19 on what kinds of patients are presenting or not presenting to the ED. In this retrospective observational study we aim to examine the changes in ED volumes during the 1st wave of the Covid-19 pandemic in Nova Scotia (NS), Canada, particularly to see if presentation patterns differed by presenting complaints.

Methods: We retrospectively investigated the utilization of 35 different EDs in NS during the 50 days before and after the declaration of the state of emergency in NS (Feb 1st to May 10th 2020) as well the same time frame from the year prior (Jan 31st to May 10th 2019). Selected presenting complaints were classified into either "Emergency" "Standard" or "Low-Acuity" using a modified Delphi consensus survey. Changes in presentations were analyzed using a 
difference in difference approach, comparing pre- and post- March 22 in 2019 with the difference in 2020, providing an estimate of the average daily change in presentations due to the Covid-19 pandemic. Results: Total ED visits in NS decreased by $40 \%$ during the pandemic time period, compared to 2019 , with 46,467 visits compared to 77,445 . Emergency, standard and low-acuity presentations decreased significantly during the pandemic time period, with decreases by $26 \%, 29 \%$ and $40 \%$ respectively. The largest decrease in ED presentation volumes happened at the beginning of the pandemic period. When presenting complaint data was separated by ED level, the level $1 \mathrm{ED}$ did not see a significant decrease in low-acuity presentations over the pandemic period (absolute daily decrease of $2.0,-13 \%$ ).

Conclusion: Our research shows that ED presentations for emergency, standard and low-acuity presentations decreased significantly in NS, identifying an unintended impact of the pandemic response. It is important that research continue to focus not only on the direct effects of COVID-19 but also on the indirect effects on non-COVID19 related morbidity and mortality. It is important that hospitals and the government continue to work on improving (1) public communication strategies to ensure that patients continue to present to the $\mathrm{ED}$ if they are having potentially serious symptoms and (2) non-ED options for care of low-acuity complaints even during a pandemic. Keywords: COVID-19, Emergency medicine

\section{P017}

Nursing initiated triage modifications have greater predictive value than computer-aided algorithms alone: cautionary data for the future of artificial intelligence at triage

S. Davis, BSc, MD, PhD, C. Ju, BSc, L. Grant, BSc, MD, PhD

McGill University, Montreal, QC

Introduction: Current triage 5-level triage systems in Canada rely on computer assisted algorithms that can then be modified by the gestalt of the nurse assessing the patient. This has raised questions of the accuracy and reliability of these triage changes. The use of artificial intelligence methods in triage has gained recent interest, so the present study aimed to assess whether human gestalt, in the form of nursing initiated uptriage, still has added value in predicting patient outcomes.

Methods: This was a retrospective analysis of 650,643 visits to a large tertiary care hospital over 19 years. All triage information was stored in a database as well as CTAS scores, and whether the patient had their CTAS score changed by the nurse. The predictor variable was a comparison between the initial computer generated CTAS score, compared the nursing changed CTAS score. Outcome variables were patient admission, and death in the ED. We then compared which score best predicted outcomes using ROC analysis.

Results: In this group, $12.6 \%$ of patients had their triage scores changed. Those with nursing changed scores were older, male, had lower pain, and were more likely to be sent to stretcher care. Comparing the AUC of the ROC curves between initial CTAS scores compared to the nursing triage score, the nursing triage score better predicted admission (AUC $=0.767$ (CI 0.763-0.772) vs AUC $=$ 0.751 (CI 0.749-0.758). The AUC predicting death was better for uptriaged CTAS scores (AUC $=0.895$ (CI 0.871-0.917) compared to non-uptriaged scores (AUC $=0.864$ (CI 0.848-0.904), and despite overlapping confidence intervals, the DeLong test of AUC differences was significant in both

Conclusion: Based on a large cohort of patients, nursing modification of triage level led to a more accurate prediction of patient admission and death than using the CTAS algorithm on its own. Given the increasing interest in $\mathrm{AI}$ at triage, the current results demonstrate that a human assessment likely still holds value.
Keywords: Patient assessment, Triage, Uptriage

\section{P018}

Human trafficking, a competency by design approach to improving recognition and management in the emergency department

J. Deutscher, MD, K. Hayman, MD, MPH, E. Dell, MD, MPH, T. Lloyd, MD, E. Fremes, MPH

University of Toronto, Toronto, ON

Innovation concept: Human trafficking is an under-recognized health and social injustice affecting patients presenting to emergency departments (ED) across Canada. Emergency physicians are in a unique position to help as the ED is often the only site of care accessed by trafficked persons. The Royal College of Physicians and Surgeons of Canada's Competency by Design aims to enhance provider skills in this area with the following Entrustable Professional Activity (EPA): recognizing and managing patients who are at risk of exposure to, or who have experienced violence and/or neglect, including human trafficking.

Methods: Kern's Model for Curriculum Development was applied in assessing need, learning objectives, method of implementation, and evaluation. A literature review explored the need for human trafficking education in emergency medicine. Curriculum mapping detected areas for introduction of novel transformative learning based material after no current content was identified in the University of Toronto emergency medicine residency curriculum. Expert organizations working with trafficked persons were consulted in the development of learning goals and material. This was piloted during resident case rounds, followed by collecting formal feedback from attendees. The final implemented material was evaluated by both learners and the expert organizations in a focus group discussion.

Curriculum, tool, or material: Learning objectives included: understanding human trafficking in Canada, recognizing the role of ED physicians, approaching screening, and managing patient care. Prior to the sessions, residents were provided access to online Canadian based education tools. The first session was a virtual lecture during Academic Day presented by a senior resident, with prior teaching expertise in this field, and representatives from local and national organizations working directly with trafficked persons. The second session was a nonmandatory simulation exercise for residents to practice screening and receive feedback from standardized patients who have extensive experience working with trafficked persons. Residents were then evaluated during ED shifts using the targeted EPA.

Conclusion: Trafficked persons commonly seek health care via the ED, positioning emergency physicians in a unique opportunity to support this vulnerable patient population. Recognizing and managing human trafficking requires skills and understanding that can be taught in collaboration with expert organizations that routinely provide care to trafficked persons.

Keywords: Competency by design, Human trafficking, Innovations in EM education

\section{P019}

Using virtual grand rounds in the emergency department to respond to the pandemic

S. Dowling, BA, MD, S. Rose, MD, F. Zaver, MD, A. Peterson, BSc, N. Solback, MSc

University of Calgary, Calgary, AB 
Innovation concept: The COVID-19 pandemic prevented all inperson gatherings starting in March 2020. The Calgary emergency department quickly pivoted their in-person academic grand rounds to a virtual format. The initial content included dissemination of rapidly changing protocols, surge planning, clinical care updates for COVID19 patients as well as interactive question and answer (Q\&A) sessions with leadership. Virtual rounds have many perceived benefits including attendee convenience, broader speaker recruitment and ease of recording sessions.

Methods: The initial virtual rounds webinar was a 90 min update, specifically addressing the changes to Calgary ED patient management as a result of the COVID 19 pandemic. In subsequent weeks, we returned to the pre-pandemic academic rounds schedule of 60-min grand rounds ED COVID-19 updates as necessary. Six international speakers were recruited for talks that would otherwise have been unobtainable.

Curriculum, tool, or material: The Zoom ${ }^{\circledR}$ webinar function enabled facilitators and participants to engage in critical updates as the pandemic evolved. A facilitator coordinated interaction via the "chat" or "(Q\&A)" functions. The webinar links were shared only with the ED email list to prevent unintended access. All sessions were recorded and posted on our ED website for those unable to attend live. On average, 140.5 people attended the live broadcasts, a significant increase from previous in-person grand rounds with a peak of 6400 views for the initial COVID update. A feedback survey sent to all ED physicians and trainees regarding the new virtual format elicited 102 responses $(58 \%$ response rate). Respondents self-report attending more rounds since going virtual and enjoying the convenience of attending from home as well as the available recordings. $100 \%$ had an overall "good/excellent" impression of the virtual format. 70.3\% said they were "much more likely to attend virtual grand rounds". Negative comments focused on missing social contact with colleagues.

Conclusion: The COVID-19 pandemic has forced medical teams to innovate their means of updating colleagues. Transitioning ED academic grand rounds to a completely online format has resulted in increased engagement, participation and convenience. It has allowed for rapid dissemination of vital and quickly changing COVID-19 protocols, and allowed access to international speakers. While challenges with online virtual rounds exist, ED's should consider its role as a long-term solution for continuing medical education.

Keywords: COVID-19, Innovations in EM education, Virtual rounds

\section{P020}

Individualized care plans: a collaborative approach to reduce frequent emergency department visitation

C. East, MD, K. Mont, B. Calder,, A. Barton, P. Anderson, MSW, S. Farquharson, MD

University of Calgary, Calgary, $\mathrm{AB}$

Background: A small population of frequent visitors make up a disproportionate number of emergency department (ED) visits and costs, and these patients may experience significant iatrogenic harms when repeatedly attending the ED. Our multidisciplinary, patientinclusive team develops individualized ED care plans for this population, using EMR flagging, information sharing, and intermittent case management. We aim to make care safe and consistent, address unmet needs in the community, and shift care out of the emergency department and into the community whenever safe and feasible. To evaluate whether our individualized care plans reduce ED visits and inpatient care admissions in a population of frequent ED visitors. Findings will be used to improve our project.
Aim statement: This is a retrospective pre/post care plan quality improvement study incorporating Calgary-Zone ED and urgent care centre (UCC) usage data from 2015 to 2020 . All patients with active ED care plans developed by our committee were included in the study. The primary outcome was patient ED visits per month. The secondary outcome was inpatient admissions per month. Average ED visits per patient per month were compared over 6 months pre-implementation, and up to 6 months post-implementation. Of the 38 included patients, 12 had recent plans with less than 6 months postimplementation data; in these cases, the data from all available postimplementation months was included (min. 1 month; additional data will be available for CAEP). Hospital admissions per patient per month were assessed over identical time periods. A Chi-squared test was used to assess the data.

Measures and design: 38 patients were included in the analysis (median age $=39$ years, range $18-96$ years, $58 \%$ female). These patients accounted for a total of $4845 \mathrm{ED}$ and UCC visits over the 5 years examined (range $0-28$ visits per patient-month). Average ED visits decreased from 4.34 per patient-month in the 6 months prior to care plan implementation, to 2.69 per patient-month in the months post implementation $(\mathrm{P}<0.001)$, and the average number of admissions decreased from 0.26 to 0.09 per patient-month $(\mathrm{P}<0.001)$.

Evaluation/results: Individualized care plans reduced ED visitation and acute care admissions for our population of frequent ED visitors. Future studies should assess impacts on ED and inpatient length of stay, advanced imaging, administration of medications with risk and abuse potential (opioids, benzodiazepines), 72-h admissions post-ED discharge, and adverse consequences such as deaths.

Keywords: Frequent emergency department users, Individualized care plans, Quality improvement and patient safety

\section{P021}

Outcomes of patients receiving renal replacement therapy in intensive care units in Nova Scotia: a retrospective database review

M. Eissa, BSc, MD, O. Loubani, BSc, MD

Dalhousie University, Halifax, NS

Introduction: Acute kidney injury is a common complication for patients in the intensive care unit (ICU), with many requiring renal replacement therapy (also known as dialysis). The decision to start dialysis often requires family and physicians to balance the possible outcomes of dialysis with the previously declared wishes of the patient. As such, an understanding of the potential outcomes of dialysis in ICU patients is needed for informed decision making. This study aims to describe mortality rates as well as the rates of patients requiring longterm dialysis in ICU patients in Nova Scotia (NS) that undergo acute dialysis. We hope this data will inform families and physicians when making decisions on starting dialysis in ICU patients in NS.

Methods: We conducted a retrospective review of data collected in the Nova Scotia provincial ICU database. We included all adult patients admitted to one of twelve adult ICUs in NS between April 1, 2018 and March 31, 2020 who received dialysis. Patients on chronic dialysis prior to admission to ICU were excluded. Hospital mortality, standardized mortality ratio, and requirement for dialysis 6 months post hospital discharge were recorded.

Results: A total of 10,027 ICU visits were recorded during the study period. Dialysis was performed in 259 ICU visits (2.59\% of ICU visits in NS). In 159 ICU visits (1.59\% of all visits), dialysis was performed in patients not previously on chronic dialysis. The APACHE IV predicted hospital mortality of patients receiving acute dialysis was 
$50.8 \%$, and the actual hospital mortality was $44.0 \%$ (70/159), representing a standardized mortality ratio of 0.87 . In patients who did not receive dialysis in ICU, predicted mortality was $15.89 \%$ and actual hospital mortality was $18.3 \%$ (1786/9768) representing a standardized mortality ratio of 1.15 . Of the patients receiving acute dialysis who survived to hospital discharge, $30.3 \%$ (27/89) required dialysis on discharge from hospital. In patients requiring dialysis at hospital discharge, $88.9 \%$ (24/27) continued to require dialysis 3 months after hospital discharge.

Conclusion: While acute dialysis is relatively uncommon in patients admitted to ICU in NS, it carries a high mortality. In those that survive, almost 1/3 require dialysis on hospital discharge, with the majority continuing to require dialysis 3 months or longer after hospital discharge. This information is important to consider for families and physicians considering starting acute dialysis in ICU patients in NS.

Keywords: Intensive care unit, Mortality, Renal replacement therapy

\section{P022}

A retrospective cohort study to determine the prevalence of serious bacterial infections in infants less than 90 days with and without symptoms or signs of a viral infection

M. Eltorki, MBChB, O. Al-Grigri, MD, A. Mirza, J. Pymento, C. Pizzola, J. Wahba, R. Carciumaru, MD, J. Pernica, MD, MSc

McMaster University, Hamilton, ON

Introduction: Prior studies suggest the prevalence of serious bacterial infections (SBI) (i.e. urinary tract infection [UTI], bacteremia or meningitis) is lower in infants with a viral infection compared to those with fever without a source (FWS) (2-3\% versus $10-15 \%)$. Our objective was to determine the difference in proportion of SBI in infants with and without clinical features of a viral infection.

Methods: A retrospective cohort study was done on a consecutive sample of infants seen at a pediatric ED over a 5-year period ending Aug 30, 2019. Eligible subjects were $\leq 90$ days old, had rectal temperatures $\geq 38 \mathrm{C}$, and had $\geq 1$ screening test for SBI (urine, blood and/or cerebrospinal fluid cultures). Excluded were infants who received antibiotics in the past 7 days, had congenital anomalies, required intensive care, or were preterm. We defined a clinical viral infection as $\geq 2$ symptoms or signs of a viral infection. UTI was defined as per Canadian Pediatric Society guidelines. Multiplex PCR panel nasopharyngeal swab results (NPS) were collected.

Results: We screened 7021 charts and 885 (12\%) were eligible. Of these, $329(37 \%)$ had FWS and $556(63 \%)$ had clinical viral infection. Blood and urine cultures were collected from $860(97 \%)$ infants and $308(35 \%)$ had a lumbar puncture. Ninety-eight (11\%) infants had an SBI: $43(7 \%)$ with clinical viral infection and $55(17 \%)$ had FWS. Among those 98 SBI, 8.6\% had UTI [95\% CI $(6.9 \%, 10.6 \%)], 2.1 \%$ had bacteremia [95\% CI $(1.4 \%, 3.3 \%)]$ and $0.3 \%$ had meningitis [95\% CI $(0.1 \%, 1.0 \%)]$. Relative Risk (RR) of UTI or bacteremia among those with clinical viral infection vs FWS were 0.5 [95\% CI $(0.3,0.7)]$ and 0.4 [95\% CI $(0.2,1.1)]$, respectively. A total of 722 (82\%) had a NPS of which $407(56 \%)$ were positive. Infants with positive NPS had a total of $13(3.2 \%)$ SBI-11 (2.7\%) UTI, $2(0.5 \%)$ bacteremia and 0 cases of meningitis. Patients with a positive NPS had lower prevalence of SBI vs. those with negative NPS. [UTI, RR $0.2 ; 95 \%$ CI $(0.1,0.4)$ and bacteremia RR $0.14 ; 95 \%$ CI $(0.03,0.6)]$. Overall proportions of contaminated blood culture and urine culture were $5 \%$ [95\% CI $(4 \%, 7 \%)]$ and $14 \%$ [95\% CI $(12 \%, 17 \%)]$, respectively, with no difference between groups.

Conclusion: SBI prevalence in infants with FWS is double that of infants with viral symptoms. SBI rates are even lower in cases of confirmed viral etiology. Contaminant blood and urine cultures are higher than true pathological cultures. Future research is needed to identify infants at low risk of SBI without invasive testing.

Keywords: Febrile infants, Severe bacterial infections, Viral illness

\section{P023}

Comparing the Ottawa emergency department shift observation tool (O-EDShOT) to the traditional daily encounter card (DEC): measuring the quality of documented assessments

K. Endres, BSc, N. Dudek, MD, MEd, M. McConnell, PhD, W. Cheung, MD, MMed

University of Ottawa, Ottawa, ON

Introduction: The Ottawa Emergency Department Shift Observation Tool (O-EDShOT) is a workplace-based assessment (WBA) designed to assess a trainee's performance across an entire shift. It was developed in response to validity concerns with traditional end-ofshift WBAs, such as the Daily Encounter Card (DEC). The OEDShOT previously demonstrated strong psychometric characteristics; however, it remains unknown whether the O-EDShOT facilitates measurable improvements in the quality of documented assessments compared to DECs.

Methods: Three randomly selected DECs and three O-EDShOTs completed by 24 faculty were scored by two raters using the Completed Clinical Evaluation Report Rating (CCERR), a previously published 9-item quantitative measure of the quality of a completed WBA. Automated-CCERR (A-CCERR) scores, which do not require raters, were also calculated. Paired sample t tests were conducted to compare the quality of assessments between O-EDShOTs and DECs as measured by the CCERR and A-CCERR.

Results: CCERR scores were significantly higher for O-EDShOTs [mean $(\mathrm{SD})=25.6(2.6)]$ compared to DECs $[21.5(3.9) ; \mathrm{t}(23)=5.2$, $\mathrm{p}<0.001, \mathrm{~d}=1.1]$. A-CCERR scores were also significantly higher for O-EDShOTs [mean (SD) $=18.5$ (1.6)] than for DECs [15.5 (1.2); $\mathrm{t}(24)=8.4, \mathrm{p}<0.001]$. CCERR items 1,4 and 9 were rated significantly higher for O-EDShOTs compared to DECs.

Conclusion: The O-EDShOT yields higher quality documented assessments when compared to the traditional end-of-shift DEC. Our results provide additional validity evidence for the O-EDShOT as an assessment tool for capturing trainee on-shift performance that can be used as a stimulus for actionable feedback, and as a source for highquality WBA data to inform decisions about emergency medicine trainee progress and promotion.

Keywords: Competency based medical education, Residency education, Workplace-based assessment

\section{P024}

A program evaluation of podiatry consult services for patients presenting to Calgary emergency departments with foot ulcers or infections

K. Exner, BSc, K. Lonergan, BSc, F. Harton, DPM, E. Lang, MD

University of Calgary, Calgary, $\mathrm{AB}$

Introduction: Although most foot complaints are managed in the emergency department (ED) solely by emergency physicians, physicians in Calgary have the unique opportunity to consult podiatrists to discuss acute management strategies and refer patients for assessment and possible admission. Evidence suggests that podiatrists play an integral role in the treatment of patients with diabetic foot ulcers (DFU) and that the involvement of podiatrists in multi-disciplinary treatment teams has had positive outcomes amongst a variety of foot 
and ankle related problems. This program evaluation aims to be the first to appraise the role of a site-based podiatry consult service in the acute care setting.

Methods: This study included all patients that presented with either a DFU or a single complicated wound of the foot or toe to Calgary EDs between 2015 and 2019. The patients were grouped by whether or not they received a podiatry consult in the ED. Comparisons were made based on several patient outcomes, including; ED length of stay (LOS), admission rates, referral rates to specialties other than podiatry (i.e. plastics, internal, etc.), rates of return within 7 days, and return ED visits requiring admission.

Results: Podiatry was consulted for 350/2208 patients. There was no significant difference between groups for average ED LOS $(523 \pm 453$ vs. $471 \pm 529 \mathrm{~min}, \mathrm{p}=0.08)$ and return rates $(13.1 \%$ vs. $11.0 \%, \mathrm{p}=0.24)$. A significantly greater proportion of patients in the podiatry arm were admitted $(38.9 \%)$, required other consults in the ED $(52.3 \%)$, and were admitted on return visits $(50.0 \%)$ as compared to the non-podiatry arm $(32.5 \%, \mathrm{p}<0.05,36.1 \%, \mathrm{p}<0.05$, and $33.8 \%$, respectively).

Conclusion: At first glance, the results seem to demonstrate that podiatry consults do not improve short-term patient outcomes in the $\mathrm{ED}$, with higher admission rates both at the index visit and at return visits within the following 7 days. However, this data, in conjunction with the need for a greater number of consults to services other than podiatry, suggests that the patients that required podiatry consults were more complex than those in the non-podiatry arm. Although a podiatry service is associated with a higher rate of admission, they provide comprehensive inpatient care for complex patients with DFU. More research would be required to tease out differences in long-term patient outcomes, such as LOS in hospital and lower limb amputation rates.

Keywords: Emergency department, Podiatry consult, Quality improvement and patient safety

\section{P025}

\section{Implementation of a sickle cell crisis protocol in a tertiary care} emergency department

M. Fu, BSc, MD, V. Thiruganasambandamoorthy, MSc, MBBS, E. Kwok, MD, MSc, MHA, S. Calder-Sprackman, MD, MSc, M. Mathieu, BSc

Department of Emergency Medicine, University of Ottawa, Ottawa, ON

Background: Pain crises are a common Emergency Department (ED) presentation of Sickle Cell Disease (SCD), with delayed management associated with more frequent hospitalization, progressive organ damage, and early mortality. Wide practice variation exists in SCD care in the ED, resulting in suboptimal outcomes for patients. The use of standardized management protocols has been shown to be effective in the treatment of other ED conditions, with improved time to treatment and patient outcomes. We aimed to evaluate whether a newly developed SCD crisis protocol would improve adherence to evidence-based best practices for the ED management of SCD.

Aim statement: We enrolled a total of 340 SCD patients presenting to a tertiary care ED with signs or symptoms consistent with a SCD pain crisis, over 2 time periods: January 2018-May 2019 (pre-intervention) and June 2019-October 2020 (post-intervention). Preintervention data was collected by health records review; post-intervention data was collected prospectively. The intervention was the iterative implementation of a protocol within the electronic medical record system using PDSA cycles. Outcome measures were: time to (1) first dose analgesia, (2) ED disposition, (3) admission. Process measures were: frequency of (1) protocol use, (2) investigations, (3) interventions. Balancing measures were: percentage of visits with (1) admission, (2) hematology consultation, among others. We performed before-and-after and run chart analyses for all measures.

Measures and design: Patients treated pre-implementation were compared to those treated post-implementation $(\mathrm{N}=190$ and 150; mean age 26.7 years and 29 years; respectively). IV fluid and oxygen use decreased post-implementation $(87.4 \%$ vs. $72 \%, \mathrm{p}=0.0004$; $58.4 \%$ vs. $32.7 \%, \mathrm{p}<0.0001$; respectively), and time to ED disposition and admission were shortened (mean difference 1.7hrs; $\mathrm{p}=0.001$; and $9.2 \mathrm{hrs}, \mathrm{p}=<0.0001 ;$ respectively). Overall protocol usage was low $(53.3 \%$ pre- and $59.5 \%$ post-implementation, $\mathrm{p}=0.26$ ). There was a shift towards decreased time to first dose analgesia on run chart analysis. No significant differences were noted in any of the other measures.

Evaluation/results: Implementing an electronic order set holds promise in optimizing evidence-based practices for SCD patients in the $\mathrm{ED}$, but alone may not be enough to address gaps in care. This study highlights the challenge of performing rapid PDSA cycles on an infrequent presentation. Strategies targeting physician education and communication may help improve protocol uptake and care practices. Keywords: Protocol implementation, Quality improvement and patient safety, Sickle cell

\section{P026}

\section{The responsibility to care: a qualitative study of emergency department workers' experiences during the COVID-19 pandemic in Canada}

N. Gaucher, MD, PhD, B. Lavoie, BA, PhD, C. Bourque, BA, PhD, A. Côté, MD, MSc, M. Rajagopal, MSc, P. Clerc, BSc, V. Bourdeau, BSc, V. Castonguay, MD, MEd, E. Fontaine-Pagé, BN, B. Burstein, MD, MPH, PhD, P. Desaulnier, MD, S. Berthelot, MD, MSc, S. Ali, MD, R. Goldman, MD, G. Thompson, MD, M. Lagacé, BN,

E. D. Trottier, MD

\section{CHU Sainte-Justine, Université de Montréal, Montréal, QC}

Introduction: The objective of this study was to prospectively explore emergency department (ED) workers' experiences during the first wave of the COVID-19 pandemic in Canada.

Methods: In this qualitative study, 6-8 workers from each of 9 Canadian EDs participated in 3 video focus groups, at 1-month intervals, to explore their experiences of the pandemic on a personal/ professional level, with regards to their work/patient care, and concerning their interactions with their teams/institutions/governing bodies. Framework analysis informed data coding and conceptual analysis.

Results: From April 14th to July 29th, 2020, 51 focus groups were conducted with 53 participants (24 physicians, 16 nurses, 13 other ED workers). Median age was 37.5 years (range 24-62), 51\% were female, $13 \%$ reported COVID comorbidity, $79 \%$ had $>5$ years' experience in healthcare. Participants in our study believed pandemic care was the continuum of emergency care: it was their responsibility to provide this care as highly specialised workers. Being provided with appropriate protection measures was essential and a significant source of concern. ED team dynamics fostered solidarity; participants trusted the ED team and management, above all, to ensure their safety. Many changes in clinical protocols and environments were implemented to protect workers and ration protection equipment; participants believed that this impacted patient care and they adapted to maintain the best quality humanized care possible. Especially in the first weeks, they received "a tsunami" of changing contradictory information and were frustrated when directives did not reflect their ED reality. Positive information management practices included 
delegating protocol developments to ED team members and designating individuals to synthesize and report important information for all shifts and workers. Participants believed each level of management had a specific role to play in facilitating their safety and quality patient care. By the end of the study, participants had adapted to a "new normality", although they were concerned about many potential sources for long-term fatigue and moral injury.

Conclusion: ED workers believe they have a responsibility to provide pandemic care but require adequate protection from infection and burn-out. To facilitate ED work, pandemic protocols should reflect and be responsive to the ED reality. Delegating protocol development to dynamic, creative and supportive ED teams is essential.

Keywords: COVID-19 pandemic, Emergency department workers, Responsibility to care

\section{P027}

Sexual assault medical clearance: a survey on the utility of an emergency department clearance tool

G. Ghate, BSc, MD, M. Clemente, BSc, MD, B. Vujcic, BSc

Western University, London, ON

Introduction: Despite an annual incidence of sexual assault (SA) of 600,000 , many emergency physicians (EPs) feel uncomfortable in managing SA victims in the emergency department (ED). Currently, a Medical Clearance tool does not exist for victims of SA for use in the ED. This study investigated the utility of a SA Medical Clearance tool for use in the ED prior to referral to a Sexual Assault and Domestic Violence Treatment Centre (SADVTC).

Methods: We surveyed a group of EPs and trainees at a large academic tertiary care ED using an online survey tool. We investigated EPs current experience with managing victims of SA, their knowledge of SA and their desire for a SA screening tool. Data was analysed with descriptive statistics.

Results: There was a total of 44 respondents (35\% response rate). The majority were Canadian trained EPs $(86 \%)$ Most $(81 \%)$ respondents assessed SA victims only a few times per year or less. Despite this, most felt very comfortable (42\%) or somewhat comfortable (36\%) assessing SA victims prior to referral to a SADVTC. However, most felt either not at all comfortable (19\%) or somewhat uncomfortable $(42 \%)$ in medically clearing SA patients without compromising evidence. There is a desire for a Medical Clearance tool, with most stating such a tool would be essential or very useful $(80 \%)$. The most important features of a tool identified include guidance on the use of lab investigations, the required aspects of a physical exam and history, the necessary ED management, and instruction on performance of an examination without disturbing evidence. The majority of respondents felt that the EP was responsible for the management of complications of pre-existing medical conditions and laceration repair, and that wound clean-up, prescription of contraception, post-exposure prophylaxis, and evidence collection were the responsibility of the Sexual Assault Nurse Examiner (SANE). Most felt a referral for psychiatric follow-up was a shared responsibility. Most respondents felt that the following were extremely important in managing SA patients: management in a quiet/private area, screening for concomitant domestic violence, risk assessment for ongoing violence and safety planning, arranging follow-up care, assessing pregnancy status, and capacity testing for informed consent for examination.

Conclusion: Our survey demonstrated that most EPs felt that a SA Medical Clearance tool would have utility in the emergency department.

Keywords: Medical clearance, Sexual assault
P028

Effect of COVID-19 on the financial transparency \& wellness of physicians and surgeons in London, Ontario

G. Ghate, BSc, MD, R. Lim, BSc, MD

London Health Sciences Centre, London, ON

Introduction: Financial transparency (FT) is the full disclosure of a business group's academic and clinical finances to all members of the group with criteria of how the finances are distribute. FT is likely important for physicians and surgeons in their workplace. Many changes were made to clinicians' financial structures across Ontario since the start of the COVID-19 global pandemic. This may have had a ripple effect on clinical business groups' FT as well as individual clinicians' financial well-being. However, there is a paucity in the literature about the importance and effect of FT on wellness and the exact components that make FT important within a business group is unclear. We sought to identify the presence or absence of FT and perceptions of FT and wellness among consultant clinicians during the COVID-19 global pandemic.

Methods: This was an online survey study aimed at describing the impact of the COVID-19 pandemic on the financial transparency practices and financial wellness among consultant physicians and surgeons across one academic centre (three sites) in London, Ontario. The survey was anonymous, consisted of 25 questions and inquired about the impact of the COVID-19 pandemic on financial transparency affecting the participant.

Results: There were a total of 122 respondents (9.4\% response rate). $111(91 \%)$ of the respondents thought that FT was 'extremely important' $58(47.5 \%)$ or $53(43.4 \%)$ 'important'. Out of the 111 respondents $75(67.6 \%)$ thought that their financial situation was negatively impacted due to COVID. Additionally, 43 (38.7\%) thought FT was of greater importance during COVID. Despite this, 64 $(57.7 \%)$ thought that their division/department did not make any financial changes to improve transparency specifically in response to the pandemic. There were no significant differences in respondents for age and gender.

Conclusion: A majority of physicians and surgeons think that FT is important and that their financial situation was negatively impacted due to the COVID-19 pandemic.

Keywords: Financial transparency, Physician financial wellness, Physician wellness

\section{P029}

Prepatching reduces paramedic in-hospital time for emergent interfacility transfers of patients requiring mechanical ventilation or vasopressors

R. Glicksman, BSc, MSc, T. Bischoff, MD, B. Sawadsky, MD, M. Peddle, MD, B. Nolan, MD, MSc

University of Toronto, Toronto, ON

Introduction: Regionalization of specialty medical services, and patient care requirements exceeding the capabilities of a local hospital may necessitate an interfacility transfer. Prepatching is a model of care adopted by critical care transport organizations to reduce time to specialty care. In this model, paramedics communicate with a transport medical physician prior to arrival at the sending hospital to discuss a patient's condition and management plan, allowing paramedics to focus solely on packaging the patient at the sending hospital. Prepatching reduces paramedic in-hospital time for emergent interfacility transfers though it remains unknown whether greater time reductions are observed in patients with higher medical needs that 
require mechanical ventilation or vasopressor support. This study sought to examine this.

Methods: We undertook a retrospective cohort study of all emergent interfacility transfers by the provincial critical care transport organization in Ontario, Canada between January 1, 2016 and December 31, 2019. We included all patients over the age of 18 years who were either intubated or on vasopressor medications. Quantile regression was used to evaluate the impact of prepatching as well as patient and paramedic characteristics on paramedic in-hospital time.

Results: A total of 4466 emergent interfacility transports were included. Of these, 1898 were completed with prepatching and 2568 were not. Vasopressor use was associated with significantly higher prepatching rates. Overall, prepatching reduced in-hospital time by $9 \mathrm{~min}$ at the 90th quantile across all patients. Increases in in-hospital time were noted for patients on mechanical ventilation, on vasopressor medications and transported by a fixed-wing vehicle by 38, 29 and $49 \mathrm{~min}$ at the 90 th quantile, respectively $(\mathrm{p}<0.05$ ). Conversely, prepatched patients transported by a critical care paramedic crew were associated with a 27-min decrease in in-hospital time at the 90th quantile compared to transport by an advanced care paramedic crew $(\mathrm{p}<0.05)$.

Conclusion: In patients that are mechanically ventilated or require vasopressors, prepatching reduced paramedic in-hospital time for emergent interfacility transports. The results of this investigation suggest prepatching can reduce overall time to definitive care in highrisk patients, potentially improving patient outcomes in critically ill patients.

Keywords: Interfacility transport, Prehospital care, Prepatching

\section{P030}

Current procedural sedation practices for adults and children in a Canadian community emergency department

A. Graystone, MD, MSc, C. Davies-Schinkel, MSc, M. Khalid, BSc, G. Hu, BSc, J. Brazier, BSc, S. Brown, MD, BMus, S. Strobel, MD, S. Upadhye, MD, MSc

Amber Graystone, Welland, ON

Introduction: Emergency Department Procedural Sedation (EDPS) is commonly used to provide adequate sedation and analgesia to patients undergoing a painful procedure. However, barriers to timely treatment often occur in busy emergency departments. The goal of this study was to examine EDPS management and length of stay measures in a Canadian community hospital ED.

Methods: A medical record review was conducted (using standardized methods) to describe the procedures requiring EDPS at a single site. Data extracted included medications administered, ED length of stay (EDLOS), and associated adverse effects. All patient age groups were included, and results were stratified to describe differences in adult vs. pediatric practices. A total of 253 patient charts were reviewed.

Results: Procedures requiring EDPS most commonly included cardioversion, orthopaedic reductions, abscess drainage, and foreign body removals. The median length of ED stay was 273 min $(95 \% \mathrm{CI}$ 250-293). The median time from triage to procedure start time was $149 \mathrm{~min}$ (95\% CI 131-163). The median duration of all reported procedures in this study was $20 \mathrm{~min}$ (95\% CI 19-22). Propofol ( \pm Fentanyl) was the most used medication, and had the shortest time from procedure start to discharge (95 min, 95\% CI 81-106). The procedures with the longest EDLOS included abscess drainage (median $365 \mathrm{~min}$ ) and foreign body removal (median $347 \mathrm{~min}$ ), which also had the longest time from triage to the procedure's start. No significant adverse effects were noted in any EDPS encounter.

Conclusion: ED procedural sedation is a common and safe, yet timeconsuming activity in community ED settings. For low acuity presentations, the greatest delay in EDLOS is incurred by prolonged preparation time after patient triage, including assembling essential personnel, equipment and monitored beds for patient placement. Use of short-acting medications and actual procedure time were not as influential on EDLOS. Opportunity exists to provide more rapid yet effective options for procedural analgesia for low acuity presentations which do not require cardiovascular monitoring.

Keywords: Analgesia, Community, Procedural sedation

\section{P031}

The state of the evidence for prehospital stroke scales: an analysis of appraised research from the prehospital evidence-based practice program

J. Greene, BSc, J. Goldstein, PhD, Y. Leroux, MD, M. Jen, MD,

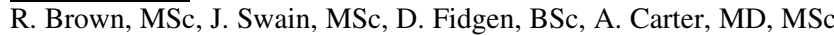
Dalhousie University, Halifax, NS

Introduction: The prehospital evidence-based practice (PEP) program is a repository of appraised emergency medical services research. Stroke is a time sensitive condition in which early detection and treatment is paramount. Prehospital $(\mathrm{PH})$ recognition by paramedics can be augmented by formalized scales, of which there are several. This is an analysis of published evidence on stroke screening scales.

Methods: PubMed is continually systematically searched. Level of Evidence (LOE) of each study was scored based on study design and quality. A Direction of Evidence (DOE) was assigned for each intervention's primary outcome. The summary LOE and DOE for each intervention were plotted in an evidence matrix.

Results: There were 47 studies describing 27 unique scales included. Eighty seven percent of the studies were conducted in the PH setting. The included scales were designed to detect stroke (47\%), large vessel occlusion (LVO) (38\%), type/severity (6\%), and definitive care or operational needs $(9 \%)$. The most studied scales were the Cincinnati Prehospital Stroke Scale (CPSS) (28\%), Los Angeles Motor Scale (LAMS) (13\%), and Rapid Arterial Occlusion Evaluation Scale (RACE) (13\%). Most studies 35/47 (74\%) were LOE II. Evidence for each scale is supportive as they each demonstrated some benefit. A 2019 systematic review reported CPSS to have the highest sensitivity for stroke identification. Of the LVO detection studies, 15/18 (83\%) investigated scales from the PH setting and (61\%) were LOE II. Two of these were systematic reviews reporting varied results. The 2017 review reports $\mathrm{PH}$ RACE scores had a sensitivity of $85 \%$ and specificity of $68 \%$ and the 2018 review reports the same predictive value for RACE but suggest the National Institute of Health Stroke Scale (NIHSS), LAMS, and vision, aphasia, neglect assessment (VAN) may have the best area under the curve (AUC) to predict LVO. Five studies investigate paramedic impression, with 4 evaluated as supportive. Paramedic impression had high sensitivity and was comparable or superior to the scale to which it was compared. The one neutral study concluded that paramedics should include a scale to improve the sensitivity of detection.

Conclusion: Many stroke screening scales are used, all providing some benefit. Paramedic impression alone can detect stroke but should be augmented by a formal scale. The CPSS is the most studied and has high sensitivity to detect stroke. The best scale to detect LVO in the PH setting is unclear.

Keywords: Emergency medical services, Paramedic, Stroke

\section{P032}

Shock and shame: international investigation of interdisciplinary emergency clinicians' pandemic-related moral injury 
H. Guertin, BSc, K. Suwary, BSc, M. Bentley, BSc, M. Whyte, D. Barbic, MD, MSc, K. Rocklein Kemplin, PhD, DNP

Western University, Windsor, ON

Introduction: Moral injury (MInj) causes professional disintegration yet remains under-quantified in emergency medicine (EM) despite its escalating suicidality. Multitudes of new studies explore MInj during the pandemic, yet few contextualize it for EM. We hypothesized that specific strata of emergency clinicians would differ in their expressions of moral injury (EMI) and couched conjectures within militaristic frameworks, given consistent clinician reports of being under siege and their anecdotes of combat.

Methods: From April-June 2020 we conducted a cross-sectional, comparative study employing the Expressions of Moral Injury Scale (EMIS). The EMIS was psychometrically normed in military populations with combat experience, which we utilized to contextualize pandemic clinical environments as battlespaces. Bias was mitigated by ensuring participant anonymity and investigator blinding of participants' geolocation and internet protocol data.

Results: Participants. In this large, international study, emergency medicine [88\% ( $\mathrm{n}=265)]$, and intensive care $[6 \%(\mathrm{n}=17)]$ were primary responses of specialty. Participants $(n=302)$ included physicians [61\% $(\mathrm{n}=184)]$, registered nurses [28\% $(\mathrm{n}=85)]$, and nurse practitioners $[5 \%(\mathrm{n}=14)]$ whose median age was 39 (IQR 32 76); $54 \%$ were female and most resided in Canada $[\mathrm{n}=183(61 \%)]$ or the United States [US; $\mathrm{n}=106(35 \%)]$. Outcomes. Primary outcome was differences in EMI between emergency clinician roles. Secondary outcomes were EMI differences among demographic categories. Analyses. Non-parametric analyses with Bonferroni corrections found significant differences $(\mathrm{p}<0.05)$ between emergency clinicians' gender, age, role, and country of practice. We reached $80 \%$ statistical power at 256 participants. Median EMIs across multiple domains were significantly higher for nurses compared to physicians $(\mathrm{p}<0.05)$, as well as for younger, and female respondents $(\mathrm{p}<0.005)$. MInj scores were significantly higher in American and British participants compared to Canada $(\mathrm{p}<0.05)$.

Conclusion: To our knowledge, this is the only investigation contextualizing EM environments as battlespaces, comparing EM physicians and nurses along specific strata. Most pandemic-related MInj studies disregard participant role, geopolitics, and generational assignment. Hence, interventions to mitigate MInj are likely imprecise and scatter-shot. These findings provide actionable evidence to develop targeted strategies as this pandemic persists and the new normal continues.

Keywords: Emergency medicine, Moral injury, Practitioner health

\section{P033}

\section{National automated external defibrillator operational readiness project: a public access defibrillation program population-based survey}

\section{Hansen, BA, J. VanDusen, K. Smith, MD, M. Douma, MN}

McMaster University, Hamilton, ON

Introduction: Public access defibrillation (PAD) programs improve outcomes of out of hospital cardiac arrest. However very little is known about the operational needs of PAD site operators to improve their readiness to respond or their willingness to co-locate naloxone. This is the first Canadian assessment of PAD site operators and barriers or facilitators to effective PAD and naloxone site operation. Methods: We identified PAD sites in Canada $(\mathrm{n}=8043)$ and randomly selected 774 , stratified using proportionate allocation by population size. Open ended survey questions as well as semi-structured interview responses were collected by telephone. Data underwent data reduction, data display and conclusion drawing using thematic analysis methods. Findings were shared with participating sites to ensure validity.

Results: Of the 774 PAD sites selected, 259 (33\%) PAD sites were successfully contacted and $169(65 \%)$ site administrators contributed data for qualitative analysis. Themes that emerged from our study included: (1) temporarily unavailable; (2) hiding in plain site; (3) cost of maintenance; (4) diffuse responsibility; and (5) willingness to respond. The majority of sites shared the effect their opening hours had on AED readiness and responder availability. Issues of signage, cabinets and other signifiers of being a PAD site were described in great detail by respondents. The most important barriers to the operators were obtaining replacement parts and consumables, as well as, not knowing who to go to for additional support in running their program. Overall, a desire to respond to emergencies, and to fulfill a duty to staff and non-staff alike was shared. PAD site operators were generally positive about co-locating naloxone and other first aid interventions. When operators voiced reluctance to co-locate naloxone they cited employer and/or administrative barriers unrelated to their individual desire to provide the intervention.

Conclusion: This qualitative inquiry gave voice to Canadian PAD site operators. The rich data obtained described the barriers and facilitators to effective PAD and naloxone site operation, in the respondents' own voices. Poignant and practical areas for PAD site improvement were identified along with areas of resourcefulness and strengths which could be further leveraged for life-saving. PAD site operators are willing to respond to emergencies, but desire resources to overcome barriers to response.

Keywords: Public safety, Automated external defibrillators, Public access defibrillation

\section{P034}

National automated external defibrillator operational readiness project: a public access defibrillation program population-based survey

M. Hansen, BA, J. VanDusen, K. Smith, MD, M. Douma, MN

McMaster University, Hamilton, ON

Introduction: Public access defibrillation (PAD) programs improve survival from out of hospital cardiac arrest. However, the readiness of automated external defibrillators (AEDs) to respond to cardiac events has not been well described. This is the first Canadian assessment of PAD AED readiness.

Methods: We identified PAD sites in Canada $(\mathrm{n}=8043)$ and randomly selected 774 , stratified using proportionate allocation by population size. PAD sites were contacted by telephone during working hours. A standardized survey was used to assess the operational readiness of their AED, challenges they experienced with AED readiness, and their attitude towards co-locating a naloxone kit with their AED. Quantitative data underwent descriptive statistical analysis to identify survey answer proportions. Qualitative responses underwent content and thematic analysis using N-Vivo.

Results: Of the 774 PAD sites selected, 259 (33\%) were successfully contacted and $217(84 \%)$ site administrators participated in the full survey and interview. Of these sites, 9 (4\%) respondents reported having used their AED during a cardiac arrest. Two hundred and ten sites (97\%) had staff onsite who were trained in using the AED. In terms of AED unit maintenance, 97\% (210) of respondents reported performing operational checks, with $161(77 \%)$ of those respondents performing checks on a monthly basis. All participants were asked to check their device's functionality, and 158 (73\%) successfully reported their device's functionality at the time of the interview. Identified barriers to AED operational readiness included a perceived 
lack of public knowledge and awareness about the PAD site and AED $(22,10 \%)$, needing more staff training $(13,6 \%)$, and a need for more equipment and assistance with setting up the AED (11, 5\%). The majority of respondents $(134,62 \%)$ responded that they would be willing to have a naloxone kit co-located alongside their AED. Those that were not willing cited lack of training, liability concerns, and cost as barriers to co-locating a kit.

Conclusion: This first Canadian survey of AED operational readiness determined that most PAD sites have operationally ready AEDs with staff available to administer resuscitative efforts. Most sites considered their AEDs to be accessible, although some referenced lack of public awareness as a limit to accessibility. Many PAD sites report willingness to co-locate naloxone kits.

Keywords: Automated external defibrillators, Public access defibrillation, Public safety

\section{P035}

A tale of two cities: comparing diagnostic imaging for emergency department patients with vertigo in Calgary and Edmonton

L. Haynes, BHSc, E. Lang, MD, C. Patocka, MD, MHPE

University of Calgary, Calgary, $\mathrm{AB}$

Introduction: Diagnostic imaging is sometimes used to distinguish peripheral vertigo from 'can't-miss' diagnoses such as posterior stroke. Peripheral and central causes of vertigo can often be distinguished using targeted physical exam maneuvers, raising the possibility that widespread use of diagnostic imaging for these patients may increase health care costs and radiation exposure with limited value added. This study sought to compare ED rates of diagnostic imaging for patients with vertigo in Alberta's two largest urban centers, Calgary and Edmonton, as well as assess whether differences in workup affected clinical outcomes.

Methods: Inclusion criteria for index visits were (1) age $\geq 20$, ED visit in the Calgary or Edmonton zone during the 2019/20 fiscal year, and (2) an ICD-10 code of R42 (dizziness and giddiness) or H81 (disorders of vestibular function). Extracted data included computed tomography angiography (CTA) or non-contrast CT (NCCT) imaging, presence of neurology consult, all-cause return ED visits within 7 and 90 days, and disposition of return visits. Proportions were compared using $\chi^{2}$ tests with $\alpha=0.05$.

Results: A comparable number of patients were seen in the ED for vertigo in Calgary $(n=4710)$ and Edmonton $(n=4774)$. The rate of CTA ordering was significantly higher in Calgary (8.7\%) than in Edmonton $(1.0 \% ; \mathrm{p}<0.001)$; however, Edmonton patients were more likely to receive a NCCT $(29.4 \%)$ than Calgary patients $(13.5 \%$; $\mathrm{p}<0.001)$. The rate of neurology consults on index visits was also significantly higher in Edmonton ( $3.0 \%$ vs $1.3 \%$; $p<0.001)$. Patients in Edmonton were more likely to have a return ED visit compared to Calgary at 7 days $(6.3 \%$ vs. $5.3 \%$; $=0.038)$ and 90 days $(22.3 \%$ vs. $19.3 \% ; \mathrm{p}<0.001)$, but there were no significant differences in the frequency of 90-day return visits for strokes or transient ischemic attacks $(0.2 \%$ vs $0.1 \%)$, nor in the frequency of admission on return visits (15\% vs $15.2 \%)$.

Conclusion: There is significant variability in diagnostic imaging practices between Calgary and Edmonton despite their seemingly similar patient populations. Although Edmonton has a higher rate of return ED visits, its lower CTA use does not appear to result in poor outcomes given both centers appear to have similar rates of return visits that result in admissions or cerebrovascular diagnoses. Increased understanding of the reasons for these differences will be important in guiding the development of a clinical pathway for care of these patients in Alberta.

Keywords: Diagnostic imaging, Dizziness, Vertigo

\section{P036}

Characterization of antibiotic prescribing practices for SARSCoV-2 positive patients discharged home from the emergency department

D. Herman, BSc, T. Rohringer, BSc, C. Graham, MD

University of Toronto, Toronto, ON

Introduction: The SARS-CoV-2 virus is known to cause COVID-19. Patients with COVID-19 presenting to emergency departments (ED) without a confirmed diagnosis at the time of presentation may receive antibiotics at discharge due to concerns for possible bacterial infection. Antibiotic prescribing practices for this population have not been well characterized. We describe antibiotic prescribing and bacterial co-infections for COVID-19 patients discharged home from two EDs at Trillium Health Partners in Mississauga, Ontario, a large multi-site community based teaching hospital.

Methods: A retrospective chart review of 200 patients with an ED visit and PCR confirmed COVID-19, randomly selected from a total cohort of 363 patients seen between January 1 and May 20, 2020. Patients requiring hospital admission, or receiving a positive SARSCoV-2 swab more than 14 days prior to the visit were excluded. Our primary outcome was the proportion of patients leaving the ED with antibiotic prescriptions.

Results: In our cohort, 35/200 patients received an antibiotic prescription, $19 / 141$ at one site and $16 / 59$ at the second $(p=0.02) .34 /$ 200 patients received prescriptions during initial ED visits with $1 / 50$ receiving a prescription at $\mathrm{ED}$ discharge on subsequent visits $(p=0.01)$. Mean age was higher in patients receiving antibiotics, 55.2 vs $47.8(\mathrm{p}=0.04)$. Respiratory symptoms and medical comorbidities were more common in patients receiving antibiotics $(24 \%$ vs $12 \%, \mathrm{p}=0.16$ and $30 \%$ vs. $16 \%, \mathrm{p}=0.10$, respectively) but the differences were not statistically significant. Of 33 patients with abnormal chest imaging 22 received antibiotics compared to $9 / 55$ with normal chest imaging (RR 4.07, 95\% CI 2.24, 7.76). No bacterial co-infection was found in 25 patients with blood and urine cultures performed in the ED. No variation in prescribing was observed over time.

Conclusion: Antibiotic prescribing in the ED was frequent in patients presenting with COVID-19, although it may be variable between hospitals and was less frequent once the diagnosis was confirmed. Antibiotic prescriptions were more frequent in patients with abnormal chest imaging, but antibiotics were still prescribed in patients without imaging features of pneumonia. No bacterial co-infections were diagnosed in any patients. Opportunities to revise antibiotic utilization during follow-up may exist in patients diagnosed with COVID-19 in the ED.

Keywords: Antibiotic stewardship, COVID-19, Pneumonia

\section{P037}

Attitudes and acceptability of organ and tissue donation registration in the emergency department: a national questionnaire of emergency physicians

M. Hickey, MD, K. Yadav, MD, MSc, K. Abdulaziz, MD, MSc, L. McIntyre, MD, MSc, H. Carly, BN, MN, J. Perry, MD, MSc, M. Hartwick, MD, MEd, A. Sarti, MD, MHPE

University of Ottawa, Ottawa, ON

Introduction: There exists a world-wide shortage of organs for transplantation. One method to increase the number of organs available for transplant is to increase the number of people who are registered organ donors. The emergency department may be a 
suitable venue to disseminate knowledge to patients about organ donation, and to offer an opportunity to register as an organ donor while they await medical care. Our objectives were to assess emergency physicians' attitudes and acceptability of utilizing the emergency department to promote organ donation registration and to examine related facilitators and barriers. We also examined physicians' support for organ donation and compare this to the proportion who are themselves personally registered for donation.

Methods: Using robust development methods, we constructed a questionnaire that was administered using a modified Dillman technique. Following cognitive interviews to optimize content and validity, it was piloted and then sent by post, in both English and French, to a random sample of 500 emergency physicians in Canada selected from the Canadian medical directory.

Results: From a total of 447 eligible respondents, we received 228 responses $(51 \%) .98 .5 \%$ of emergency physicians support the concept of deceased organ donation. $85.1 \%$ felt that the emergency department is an appropriate setting to disseminate information regarding organ donation and $77.6 \%$ felt that it is an appropriate location to offer an immediate opportunity to register as an organ donor. $65.4 \%$ of physicians who responded report to be personally registered as an organ donor. Several facilitators and barriers were identified that may impact organ donation registration in the emergency department.

Conclusion: The majority of emergency physicians in our study are engaged in supporting organ donation. The emergency department is an acceptable venue to consider promoting organ donation registration but the mechanism to achieve this is less clear and requires further study.

Keywords: Organ donation, Postal survey

\section{P038}

\section{Attitudes and acceptability of organ and tissue donation registration in the emergency department: a national questionnaire of emergency nurses}

M. Hickey, MD, L. McIntyre, MD, MSc, J. Perry, MD, MSc, K. Yadav, MD, MSc, C. Hickey, BN, MN, M. Hartwick, MD, MEd, A. Sarti, MD, MHPE

\section{University of Ottawa, Ottawa, ON}

Introduction: The global supply of organs for transplantation outweighs the supply. One strategy to address this issue is to expand the means by which citizens become registered organ donors. Given the large volume of patients that visit the emergency departments, there may opportunities to educate and register patients and visitors for organ donation while they await medical care. Emergency nurses are key stakeholders in emergency department operations and their engagement would be important in this novel initiative. We aimed to assess emergency nurses support for organ donation and to examine their attitudes and acceptability of an emergency department-based organ donation registration strategy.

Methods: This study was a cross-sectional, electronically administered survey of Canadian emergency nurses. It was distributed to all 1270 active members of the National Emergency Nursing Association. The survey instrument was developed using robust methodology and included stakeholder and cognitive interviews. Following piloting and final adjustment, it was distributed nationally in both English and French languages.

Results: Of 1270 nurses contacted, there were 130 responses from eligible participants, resulting in a response rate of $10.2 \%$. The majority of responses were from nurses practicing in Ontario, Alberta and British Columbia. $80 \%$ of nurses reported being personally registered as organ donors. The majority of respondents are generally supportive and engaged in organ donation and felt that the emergency department is an appropriate venue to disseminate information about organ donation. Some nurses felt that patients would not be open to receiving information regarding organ donation, and several facilitators and barriers such a strategy were identified.

Conclusion: Emergency nurses have a positive attitude and are engaged in organ donation. The majority of respondents in our study are supportive of utilizing the emergency department to educate the public and to offer an opportunity for patients to register as an organ donor. However, further investigation is required to construct the optimal mechanism for implementation.

Keywords: Organ donation

P039

Appropriateness of emergency physician antibiotic prescribing for uncomplicated purulent skin and soft tissue infections

T. Ibrahim, MSc, C. Thompson, MSc, B. Borgundvaag, MD, PhD, S. McLeod, MSc, PhD

Schwartz/Reisman Emergency Medicine Institute, Sinai Health, Toronto, ON

Introduction: Purulent skin infections are a common cause of emergency department (ED) visits. Current guidelines suggest a weak recommendation for the use of trimethoprim/sulfamethoxazole (TMP-SMX) or clindamycin in addition to incision and drainage (I\&D) alone. To limit bacterial resistance and harms associated with antibiotic use, antimicrobial stewardship encourages antibiotic prescribing only when necessary. The objective of this study was to describe the current common bacteria associated with purulent skin infections and appropriateness of antimicrobial selection in the ED.

Methods: This was a retrospective medical record review of adult patients ( $\geq 18$ years) discharged from an academic hospital ED (annual census 65,000) over a 2-year period with diagnosis of an uncomplicated skin abscess. Patients were excluded if they were febrile, immunocompromised, or their infection was related to hospital admission or recent surgery. Using a study-specific data extraction tool, trained research staff recorded culture and sensitivity $(\mathrm{C} \& S)$ results where applicable and antibiotics prescribed at ED discharge.

Results: Of 389 cutaneous abscesses visits, 152 (39.2\%) had a C\&S analysis performed, with $111(73.0 \%)$ cultures being positive for pathogens. Of the positive cultures, $71(64.0 \%)$ grew S. aureus, of which $32(45.1 \%)$ were MRSA. Of the $238(61.2 \%)$ patients who received antibiotics at discharge, $85(35.7 \%)$ received guideline compliant antibiotics (TMP-SMX or clindamycin), and in total 110 $(46.2 \%)$ received an antibiotic effective against MRSA. In total, 53 $(47.7 \%)$ patients received an antibiotic at discharge that was effective against the isolated pathogen. Of the $58(52.3 \%)$ patients who were discharged without an effective antibiotic based on their $C \& S$ results, $21(36.2 \%)$ of which were switched to an appropriate antibiotic via a post-discharge quality assurance (QA) call-back program.

Conclusion: It is unclear if every patient with an uncomplicated abscess requires antibiotics, however, if antibiotics are to be prescribed, current guidelines suggest using those effective against MRSA. We found $61.2 \%$ of patients were prescribed an antibiotic at ED discharge, however in less than half of these cases, an antibiotic that was guideline compliant was prescribed. Given the harms associated with inappropriate antibiotic prescribing, further research is required to understand why prescribing practises do not adhere to current guidelines.

Keywords: Antibiotic, Emergency department, Skin and Soft tissue infections 


\section{P040}

Can plain film radiography improve the emergency department detection of clinically important renal stones

G. Innes, MD, MSc, I. Wishart, MD, T. Lau, MD, K. Gourlay, BSc, A. Islam, BSc, F. Scheuermeyer, MD, MSc

University of Calgary, Calgary, $\mathrm{AB}$

Introduction: Ultrasound has limited sensitivity for renal stones and rarely specifies stone location or size. Despite advances in emergency department (ED) point of care ultrasound (POCUS) use, CT rates have continued rising, and up to $80 \%$ of patients with suspected renal colic now undergo CT imaging. X-ray is rarely performed because of poor sensitivity, but it is rapid, inexpensive, low radiation and available in low-resource settings. We investigated whether adding $X$ ray to hydronephrosis assessment could improve detection of large stones and stones likely to benefit from early intervention.

Methods: We reviewed imaging reports from all patients with a renal colic diagnosis at four urban EDs who underwent X-ray and computed tomography (CT) during their ED visit. We classified stones by size and location and identified those that were detected on X-ray. We concurrently reviewed CT reports and documented the degree of hydronephrosis seen. Because mild hydronephrosis is unreliable and nonspecific, we considered moderate or severe hydronephrosis (MSHydro) to be significant positive findings. We calculated the sensitivity (detection rate) of X-ray and MS-Hydro, respectively, for large stones $>5 \mathrm{~mm}$ and for stones likely to require intervention. Based on recent research, we defined interventional stones as all ureteral stones $>7 \mathrm{~mm}$ and proximal or middle stones $>5 \mathrm{~mm}$. We then tested the performance of a diagnostic algorithm that combined MSHydro detection plus X-ray.

Results: We studied 1026 patients with 1527 stones. Overall X-ray sensitivity was poor, only $31.3 \%$; however this increased to $45.6 \%$ for large stones and $51.6 \%$ for interventional stones. MS-Hydro identified $38.8 \%$ of large stones and $60.2 \%$ of interventional stones. Adding Xray to MS-Hydro detection substantially increased sensitivity for all stone categories, notably from 60.2 to $81.7 \%$ for interventional stones, an absolute gain of $21.5 \%$; $95 \%$ CI $13.3-29.7 \%$ ), and from 38.8 to $68.0 \%$ for large stones $>5 \mathrm{~mm}$, an absolute gain of $29.2 \%(95 \% \mathrm{CI}$ $23.3-35.1 \%)$.

Conclusion: The combination of plain X-ray and hydronephrosis assessment detects nearly $70 \%$ of large stones and over $80 \%$ of interventional stones. This level may be sufficient to reassure physicians and patients about the safety of a wait-and-see approach without $\mathrm{CT}$ imaging in many cases.

Keywords: Diagnosis, Diagnostic imaging, Renal colic

\section{P041}

Not just the asthma: understanding acute asthma experiences of emergency department patients through photovoice

L. Krebs, BA, MSc, BSW, MPP, N. Hill, MA, C. Villa-Roel, MD, PhD, P. McLane, PhD, B. Rowe, MD, MSc, S. Gupta, MD, MSc

University of Alberta, Edmonton, $\mathrm{AB}$

Introduction: Asthma exacerbation is a frequent reason for emergency department (ED) visits, yet few qualitative studies explore asthma acute care experiences. No studies have explored this using visual methods.

Methods: We used photovoice methods to explore adult patient experiences of asthma exacerbation necessitating ED care. Photovoice involves small sample sizes of participants taking and interpreting photos to convey their experience, allowing development of a rich visual and interview dataset by and for each participant. Using purposive sampling, we recruited patients aged 17-55 years presenting to the ED for acute asthma. Participants had 4 weeks to take photographs, followed by a one-on-one photo-elicitation interview. Interviews were audio recorded, transcribed, and were thematically analyzed together with the photograph data.

Results: Six patients were recruited; two were lost to follow-up. One primary theme emerged: the roles that hope and fear play in the lives of participants and how these emotions came to the fore during an exacerbation. Participants described the tension between a constant need to maintain hope to sustain them against the fear that threatened to overwhelm that hopefulness. Hope took various forms: maintaining a hopeful mindset; reminding themselves of the temporary nature of the exacerbation; and/or finding a provider that listened and understood them. Fear was also embedded in their experiences of: the severity of their symptoms; the way their condition limited their lives; and concerns that they would find limited help or understanding when seeking care. The primary theme also permeated identified subthemes: (1) need to adopt the roles of being both advocate and expert; (2) frustration with their health state; (3) being constrained by their physical health and the toll this took on their mental health; (4) barriers to accessing appropriate and timely health care; and (5) their experiences of "good" and "bad" ED care.

Conclusion: Participants described the complexities of having a chronic condition, permeating their lives during times of 'good asthma control' and exacerbation. Participants' focus on the roles of hope and fear, both in the ED and beyond, suggests that openly acknowledging and addressing these emotional aspects as a part of the ED interaction should be considered by clinicians. Such an approach may enable clinicians to better see the whole person and to address their, at times, unexpressed needs.

Keywords: Acute asthma, Patient experience, Photovoice

\section{P042}

More than just a headache: understanding the experiences of headache-sufferers who attend the emergency department through photovoice

L. Krebs, BA, MSc, BSW, MPP, N. Hill, MA, C. Villa-Roel, MD, $\mathrm{PhD}, \mathrm{B}$. Rowe, MD, MSc

University of Alberta, Edmonton, AB

Introduction: Headaches are a common presenting problem to emergency departments (ED) in Canada. Current qualitative research highlights that headaches impact all aspects of peoples' life: physical, relational and emotional. This study set out to explore the lived experience of individuals suffering with benign headaches who seek care in the ED using visual methods.

Methods: We employed the photovoice method to explore patient experiences. Typically, photovoice is conducted with a small number of participants who take and interpret photos to convey their experience. Use of this method develops a rich visual and interview dataset by and for each participant. A purposive sample of patients who presented to two ED sites in Edmonton who were provided approximately 3 weeks to take photographs, and then complete a 60 $90 \mathrm{~min}$ one-on-one, in-person photo-elicitation interview. Interview transcripts were thematically analyzed alongside the photographs.

Results: Eight participants completed the study. Their data revealed five themes, specifically: (1) the struggle for legitimacy in light of the invisibility of their condition; (2) the importance of hope, hopelessness and fear in the day-to-day life and emotional reality of participants; (3) the importance of agency and becoming "you own advocate" within the ED and beyond; (4) the struggle to be and be seen as themselves despite the encroachment of their headaches; and 
(5) the realities of "good" and "bad" care in the ED. Participants highlighted examples of good care in the ED, specifically when they felt seen and believed. Additionally, some expressed the acute care space itself being a beacon of hope in the midst of their crisis. Participants conceptualized bad" care, as when they "feel neglected" and alone. Some reported feeling dismissed because providers "know it's not life or death".

Conclusion: This study demonstrated the feasibility of conducting a photovoice study with patients presenting to the ED with a chronic condition and allowed participants to share the complexity of seeking ED care. This study highlighted the substantial emotional impact that benign headaches have on participants life. Additionally, this study identified specific themes that should be the target of actions within the ED care context to help ensure that patients feel treated as a whole person and their concerns are addressed.

Keywords: Benign headache, Patient experience, Photovoice

\section{P043}

Tough medicine: experiences of clinicians providing palliative care in the emergency department

L. Krebs, BA, MSc, BSW, MPP, N. Hill, MA, S. Kirkland, MSc, C. Villa-Roel, MD, PhD, A. Elwi, PhD, B. O’Neill, BScN, S. Duggan, $\mathrm{MD}$, A. Brisebois, MD, MSc, B. Rowe, MD, MSc

University of Alberta, Edmonton, AB

Introduction: Providing end-of-life (EOL) and palliative care (PC) in the chaotic, fast-paced, emergency department (ED) is challenging, particularly given the ED's provision of temporary management aimed at facilitating recovery. This study explored emergency physicians and nurses experiences in providing PC.

Methods: Emergency physicians and nurses were recruited. Physicians were purposively selected, based on demographics, including: site of practice, years of practice, sex, and level of certification. A convenience sample of nurses was undertaken. One-on-one, semistructured interviews were audio recorded and transcribed verbatim. The interview guide addressed conceptualization of PC and roles, as well as challenges and opportunities in ED-based PC. An inductive thematic analysis was completed.

Results: Eleven physicians and four nurses were interviewed. Clinicians saw PC as "a spectrum... not just your last days" and the ED's care focus on "controlling symptoms." Physicians saw themselves as "temporary quarterbacks" of care while nurses saw their role as providing information and advocacy. Conversations were the core activity of PC in both groups. Clinicians expressed frustration that patients had often been poorly informed about their condition prior to presentation, which contributed to feeling responsible for ensuring they understood their condition/prognosis at discharge. Lack of a preexisting relationship complicated these conversations causing concern that "we can do harm to people by not stewarding those conversations well." Families could ease conversations or further complicate them, particularly when a lack of consensus around goals of care existed. Clinicians felt the ED was "not an ideal place" for PC and that the ED could not provide the environment for a dignified death. This was juxtaposed by several clinicians feeling that death was "sacred" and wanting to preserve the "holy moment" of "midwifing... people from life to death". Clinicians also identified system failures, such as lack of time, lack of resources or difficulty in accessing resources, and a lack of training in PC. Finally, some clinicians described the complexity of caring for patients at EOL and the impacts it had on them personally.

Conclusion: Conversations and resource availability were common challenges in providing ED-based PC. Accessing additional education and streamlining referral procedures were proposed as possible interventions to improve the ED journey.

Keywords: Clinician experiences, End-of-life, Palliative care

\section{P044}

A scoping review to identify access barriers and knowledge gaps for 2SLGBTQ+ patients in the emergency department

M. Kruse, BSc, MD, B. Bigham, MD, MSc, D. Voloshin, MD, S. Upadhye, MD, MSc

Queens University, Kingston, ON

Introduction: Sexual and gender minorities (hereafter 2SLGBTQ+) make up 2.3-8\% of the North American general population, and face specific higher chronic illness burdens and challenges accessing healthcare. There are barriers to accessing and receiving appropriate Emergency Department (ED) care variably described in the literature. The goal of this scoping review was to collate and summarize the current literature addressing ED 2SLGBTQ+ care, identify barriers and knowledge gaps, and propose solutions to these gaps.

Methods: Using PRISMA-ScR (Scoping Review) criteria, we conducted an electronic search (CINAHL, Embase, Ovid Medline and Web of Science) for all studies of involving 2SLGBTQI+ populations published after 1995, and throughout all life stages, presenting to an ED in North America. We included articles with patients of all ages in North American ED's, and all study designs. We excluded non-North American studies, reports not based on ED care, editorials and conference abstract presentations. Retrieved titles and abstracts were screened independently, and full text review was performed with two reviewers. Abstraction focused on study design, demographics, and outcomes, and the resulting data was analysed using an iterative thematic analysis.

Results: The search strategy identified 703 articles for review, with 149 duplicates. Articles ( $\mathrm{n}=554)$ were screened for title and abstract, and 426 articles were excluded. The remaining 128 articles underwent full text review, and 56 articles were included in the final count. Common themes identified were disease epidemiology (17), HIV in SGM (14), ED use (10), ED avoidance or barriers (9), EM provider training (9), and sexual orientation/gender identity information collection (8).

Conclusion: The current literature encompassing ED 2SLGBTQI+ care clusters into 6 themes, with variable exploration of each. There are considerable gaps to be addressed in optimizing culturally competent and equitable care in the ED for this population. Future research to address these gaps should include substantial patient stakeholder engagement in all aspects of the research process to ensure patient-focussed outcomes congruent with 2SLGBTQI+ values and preferences.

Keywords: 2SLGBTQ+, Access to care, Scoping review

\section{P045}

Does direct observation happen early in a new competency-based residency program?

\section{J. Landreville, MD, J. Frank, MD, MEd, W. Cheung, MD, MEd}

University of Ottawa, Ottawa, ON

Introduction: A key component of competency-based medical education is workplace-based assessment which includes observation (direct or indirect) of residents. Direct observation has been emphasized as an ideal form of workplace-based assessment yet challenges have been identified which may limit its implementation. At present, 
it remains unclear how often direct and indirect observation are being used within the clinical setting. The objective of this study was to determine the frequency of direct and indirect observation in a competency-based residency program 2 years post implementation. Methods: Emergency medicine residents $(n=19)$ were asked to record the type of observation they received (direct or indirect) following workplace-based entrustable professional activity assessments from December 15, 2019 to April 30, 2020. All completed assessment forms were collected and reviewed. Descriptive statistics were performed to determine the frequency of each observation type.

Results: Assessment data was collected on all 19 eligible residents (100\% participation rate). A total of 1070 entrustable professional activity assessments were completed during the data collection period, of which 798 (74.6\%) had the type of observation recorded. Of the assessments with observation type recorded, $546(68.4 \%)$ were directly observed and $252(31.6 \%)$ were indirectly observed.

Conclusion: To our knowledge, this study is the first to report frequency of direct and indirect observation in a competency-based residency program. The results of this study suggest that direct observation can be quickly adopted and implemented as the primary means of gathering workplace-based assessment data. Indirect observation comprised a sizeable minority of recorded observations and may be an underrecognized contributor to workplace-based assessment.

Keywords: Competency based medical education, Observation, Workplace-based assessment

\section{P046}

Contrasting the impact of the COVID-19 pandemic on emergency medical services and emergency department frequency, presenting severity, disposition, and mortality

D. Lane, PhD, A. McRae, MD, PhD

University of Calgary, Calgary, AB

Introduction: Emergency Medical Services (EMS) and the Emergency Department (ED) are the first points of contact with the health care system for many patients. During the early period of the COVID19 pandemic, EMS systems reported a decrease in the number of EMS responses, while EDs reported a decreased volume of patients. At the same time, evidence emerged of an increase in non-COVID 19 out-of hospital deaths and a $20 \%$ excess mortality between March and July 2020. This study quantified the frequency, presenting severity, disposition and mortality of patients presenting to EMS and the ED during the early period of the COVID-19 pandemic.

Methods: A population cohort of all patients accessing emergency services, either by Emergency Medical Services (EMS) or presenting directly to an emergency department (ED) or urgent care centre (UCC) was constructed. Two historical control periods (December to June, 2017/2018, 2018/2019) were compared to one exposure period (December to June, 2019/2020). Time-series analysis was used to examine changes over the course of the pandemic.

Results: A total of 1,127,014 patient encounters were included. There was a $40 \%$ increase in patients presenting to EMS during the exposure period, and a $40 \%$ decrease in patients presenting to the ED/UCC. The proportion of EMS calls for the most severe patients remained stable, while the proportion of most severe ED patients increased by $49 \%$. Paramedics spent longer on scene with patients (17\% increase) and ED physicians spent longer attending to patients (10\% increase), despite shorter ED wait times (51\% decrease) and boarding times ( $11 \%$ decrease). A greater proportion of ED patients were admitted to hospital (20\% compared to $17 \% ; 20 \%$ relative increase). The daily mortality rate among EMS patients increased 3.3-fold (12 deaths compared to 3.6 per 1000 patient encounters) following the first COVID-19 case.
Conclusion: The COVID-19 period was associated with clinically and operationally significant changes in the frequency, severity and disposition of emergency patients. Further research will elucidate the mechanism of these observations as is critical to mitigating the impact of ongoing COVID-19 system burden.

Keywords: COVID-19, Emergency department, Emergency medical services

\section{P047}

Examining patient and contextual factors predicting delirium recognition in 1477 older emergency department patients in a national bilingual multicenter trial

J. Lee, MD, MSc, T. Tong, PhD, M. Chignell, PhD, M. Tierney, PhD, J. Goldstein, PhD, D. Eagles, MD, MSc, J. Perry, MD, MSc, A. McRae, MD, MSc, E. Lang, MD, MSc, L. Rose, PhD, D. Melady, MD, MEd, B. Borgundvaag, MD, PhD, S. McLeod, PhD, A. Kiss, $\mathrm{PhD}$, V. Boucher, MSc, M. Emond, MD, PhD

\section{Schwartz/Reisman Emergency Medicine Institute, Toronto, ON}

Introduction: Despite increasing mortality tree fold, unrecognized ED delirium remains common. We recently showed that delirium recognition remains below 50\% in the ED. Thus we set out to assess patient and contextual factors predicting delirium recognition by ED nurses and physicians.

Methods: This is a planned sub-study of a multi-center, randomized clinical trial which enrolled people $\geq 65$ years old, excluding those with an ED stay $<4 \mathrm{~h}$, critical illness, or from a nursing home. We prospectively assessed: (1) age, (2) gender, (3) frailty using the Canadian Frailty Scale (CFS), (4) Charlson Comorbidity Index (CCI), (5) delirium severity using the validated 21-point delirium index, (6) arrival by ambulance, and (7) site. Research assistants (RA) prospectively assessed delirium using the validated Confusion Assessment Method (CAM), and then asked patients' ED nurse or physician whether their patient had delirium. ED nurses and physicians were asked to assess delirium using their standard clinical approach at the beginning of the study. We conducted bivariate analysis (Chi square) and multivariable logistic regression with forward selection to assess potential predictors, using the 10 to one rule of thumb to determine number of predictors assessed.

Results: We had CAM assessments on 1477/1493 (98.9\%) of participants; their mean age was $76.5 ; 49.2 \%$ were female; $46.0 \%$ arrived by ambulance; mean CFS was 3.47; and $79(5.2 \%, 95 \%$ CI $4.2-6.5 \%)$ had delirium. Bivariate analysis showed that arrival by ambulance $\left(\chi^{2}\right.$ $\mathrm{df} 1=4.54, \quad \mathrm{p}=0.033)$ and delirium severity $\left(\chi^{2} \quad \mathrm{df} 1=5.54\right.$, $\mathrm{p}=0.018)$ predicted delirium recognition. Only patients' delirium severity predicted delirium recognition in multivariable analysis (Wald $\chi^{2}=8.97, \mathrm{p}=0.003$ ). The odds of recognizing delirium increased by 1.25 for each unit increase in delirium index $(95 \% \mathrm{CI}$ $1.09-1.48$

Conclusion: Our hypothesis-generating findings suggest that ED nurses and physicians are less likely to recognize patients with less severe delirium. Future research should focus on methods to identify those with subtle delirium.

Keywords: Delirium recognition, Geriatric emergency medicine, Predictors

\section{P048}

Experiences of healthcare providers with a novel emergency response intubation team (ERIT) during the first wave of the COVID-19 pandemic 
D. Lee, BSc, S. Crump, MScN, L. Chartier, MD CM, MPH, P. Perri, BScN, M. Parotto, MD, PhD, M. Ma, MD, K. Chin, MD, MMed, K. Nirmalanathan, MSc, S. Sabbah, MD, MSc, A. Taher, MD, MPH

University of Toronto, Toronto, ON

Introduction: The intubation of COVID-19 patients confers significant safety risks to healthcare providers (HCPs). In response, a dedicated Emergency Response Intubation Team (ERIT) consisting of anesthesiologists and allied health providers was instituted for our emergency department (ED). Given the high-risk nature of intubations and the new interprofessional team dynamics, we sought to assess HCP experiences and potential areas of improvement.

Methods: Surveys were distributed to HCPs at University Health Network, a quaternary healthcare centre in Toronto, Ontario, which includes two urban EDs seeing over 115,000 patients per year. Participants included ED physicians and nurses, anesthesiologists, anesthesia assistants, and operating room nurses. The survey consisted of Likert-scale and free-text questions. Likert-scale questions were analyzed with descriptive statistics. Free-text responses underwent thematic analysis using grounded theory and were independently coded by two authors to generate descriptive themes. Discrepancies were resolved with a third author. Descriptive themes were distilled through an inductive, iterative process until fewer main themes emerged.

Results: A total of 178 surveys were collected (68.2\% response rate). Of these, $123(69 \%)$ participated in two or more ERIT activations. Prior to their ERIT involvement, HCPs reported participating in simulation training, having no formal training, or referring to written content (e-mails, memos) or huddle discussions. Positive aspects included increased numbers of staff to assist, increased intubation expertise, efficient transfers to the intensive care unit (ICU), improved safety, and good team dynamics within the ERIT team. Perceived challenges included a loss of scope (primarily ED physicians and nurses) and unfamiliar work flows, delays to ERIT team arrival or patient intubation, role confusion, handover concerns, and communication challenges between ED and ERIT teams. Perceived opportunities for improvement included interprofessional training, developing clear guidelines on activation, inter-team role clarification, and guidelines on handover processes post-intubation.

Conclusion: HCPs perceived that a novel interprofessional collaboration for intubations of COVID-19 patients presented both positive gains and challenges. There were perceived opportunities for improvement. Further research is needed to delineate best practices. Keywords: Interdisciplinary health team, Intubation, Rapid response team

\section{P049}

A population-based observational study on the effects of the COVID-19 pandemic on a large Canadian community emergency department

D. Lee, BSc, H. Jung, MSc, W. Lou, PhD, D. Rauchwerger, MD, L. Chartier, MD CM, MPH, S. Masood, MD, MPH, S. Sathiaseelan, $\mathrm{MD}, \mathrm{A}$. Taher, MD, MPH

University of Toronto, Toronto, ON

Introduction: As the first wave of the COVID-19 pandemic unfolded, emergency departments (EDs) across the world braced for surges in volume and demand. However, many EDs experienced decreases in volumes, even for higher acuity illnesses. Our study examines ED utilization at a large Canadian community hospital, including changes in patient demographics, presentations, as well as structural and administrative changes made in response to the pandemic.
Methods: This retrospective observational study took place at Mackenzie Health ED in Richmond Hill, Ontario, from March 17 to June 30, 2020, corresponding to a province-wide lockdown in response to COVID-19. Expected values were calculated using an average of historical data during the same dates in 2018-2019. Differences between observed and expected values were calculated for total visits, Canadian Triage and Acuity Scale (CTAS) groups, and age groups with Fisher's exact test. Length of stay (LOS), physician initial assessment (PIA) time, and top primary and admission diagnoses were compared with descriptive statistics.

Results: Patient visits fell to $66.3 \%$ of expected during the exposure period $(20,901$ vs. $31,525, \mathrm{p}<0.0001)$. CTAS-1 (highest acuity) patient volumes were $86.8 \%$ of expected $(p=0.20)$ while CTAS-5 (lowest acuity) patient volumes were $32.4 \%$ of expected ( $\mathrm{p}<0.0001)$. Youth $(0-17$ years), adult (18-64 years), and senior (65 + years) visits decreased to $37.4 \%, 71.7 \%$, and $72.9 \%$ of expected volumes, respectively (all $\mathrm{p}<0.0001$ ). Median PIA and median ED LOS both decreased (1.1-0.6 h and 3.3-3.0 h, respectively). The most common primary diagnosis in both periods was "other chest pain". Viral illnesses were more prevalent in the exposure period. The top admission diagnoses were COVID-19 in the study period $(3.5 \%)$ and congestive heart failure in the control period (4.8\%). This ED responded with human resource changes (agile ED staffing, redistributing staff to COVID-19 assessment centre), spatial reorganization (refitting low-acuity treatment zone to high-acuity patient isolation zones, removing beds to allow for physical distancing), and improved infection control (HEPA ventilation, full-spectrum UV disinfection machines).

Conclusion: ED utilization changed significantly during COVID-19. The experiences of this local ED experiences can help equip ED administrators with structural and process-based changes for potential subsequent "waves" of COVID-19 and future pandemics.

Keywords: COVID-19, Emergency department, Patient volumes

\section{P050}

Undertriage of severely injured trauma patients: a review of interfacility transfers of trauma patients meeting field trauma triage standards

W. Li, MD, MSc, G. Mok, MD, B. Nolan, MD, MSc

University of Toronto, TORONTO, ON

Introduction: Optimizing prehospital triage of injured patients to the appropriate level of care is an essential component of a trauma system. In Ontario, paramedics use the Field Trauma Triage Standards (FTTS) to identify patients at risk for severe injury. The FTTS criteria encompasses physiologic, anatomic, mechanism of injury, and special considerations to identify patients that should be transported directly to a trauma centre. Undertriage, whereby patients are not taken directly to a trauma centre can occur if the FTTS was not sensitive enough or if the FTTS was not followed appropriately. This results in delays to definitive care and has been associated with patient harm. The objective of this study was to evaluate the appropriate application of FTTS in severely injured trauma patients that underwent interfacility transport to a level 1 trauma center.

Methods: We conducted a retrospective cohort study of all interfacility transfers of trauma patients to a level 1 trauma center over a 3year period that were either admitted to the intensive care unit, received an operation within $4 \mathrm{~h}$ of arrival, or died within $48 \mathrm{~h}$ of arrival. Data were abstracted from the hospital's trauma registry with supplemental information obtained from manual chart review of electronic medical records. Two reviewers then determined whether patients did or did not meet FTTS. 
Results: There were 3327 trauma patients during the study period of which 1008 were interfacility transfers. In total 340 patients met our inclusion criteria. Of the study cohort, $78.5 \%(n=267)$ of interfacility transports had met at least one FTTS criteria. The most frequent FTTS criteria met were: Glasgow Coma Scale $<14$ (42.4\%), high risk motor-vehicle collision $(22.1 \%)$, and systolic blood pressure $<90 \mathrm{mmHg}(19.4 \%)$. The median intensive care unit length of stay was 10 days for patients that had met FTTS vs. 2.5 days for patients that did not meet FTTS. In-hospital mortality was $16.5 \%$ for those who met FTTS vs. $8.5 \%$ for those who did not meet FTTS.

Conclusion: More than $78 \%$ of critically injured trauma patients transported to a level 1 trauma centre as interfacility transfers had met initial FTTS criteria necessitating direct bypass to a trauma center. These patients are at high risk for preventable morbidity and mortality. This study indicates the need for ongoing for education and adherence to prehospital trauma triage protocols.

Keywords: Patient safety, Prehospital, Trauma

\section{P051}

\section{Diagnosis and management of wide complex tachycardia in the emergency department}

J. Linton, BSc, D. Eagles, MD, MSc, S. Alchi, BSc, M. Nemnom, MSc, M. Green, MD, I. Stiell, MD, MSc

Department of Emergency Medicine, University of Ottawa, Ottawa, $\mathrm{ON}$

Introduction: While wide complex tachycardia (WCT) is common and potentially lethal, little is known about its incidence in the ED or about expertise of ED physicians in diagnosing and treating it. We sought to evaluate the demographics of WCT as well as the accuracy of ED diagnosis and appropriateness of treatment.

Methods: We conducted a health records review at a large academic hospital ED staffed by 95 physicians and 55 residents and included patients seen from June 2018 to September 2020 with an ED ECG showing WCT [heart rate $(\mathrm{HR}) \geq 120 \mathrm{bpm}$ and $\mathrm{QRS} \geq 120 \mathrm{~ms}$ ]. Data were abstracted from an electronic medical record onto a case record form by 2 medical students and reviewed by 2 ED physicians and 1 cardiologist. Cases were adjudicated for the accuracy of ECG diagnosis versus the cardiology read and for correctness of treatment as per guidelines. Data analysis entailed descriptive and univariate statistics.

Results: We included 306 cases who were mean age 73.8 years, male 66.0\%, median HR 132 (maximum 227), SBP < 90 15.4\%; admitted to a monitor $44.4 \%$ or a ward $9.2 \%$, discharged home $42.5 \%$, died in ED $2.3 \%$. WCT was a primary arrhythmia $45.4 \%$, secondary to medical illness $46.7 \%$, and both $7.8 \%$, with the most common medical diagnoses: infection $29.3 \%$, heart failure $17.1 \%$, acute coronary syndrome $13.4 \%$. ED physicians were correct in ECG interpretation for $72.5 \%$ and in identification of primary vs secondary WCT in $97.4 \%$. The most common primary arrhythmias and \% correct ED reading were: atrial fibrillation $39.5 \%(95.0 \%)$, atrial flutter $17.3 \%$ $(62.5 \%)$, sinus tachycardia $16.3 \%(67.9 \%)$, uncertain diagnosis $11.8 \%$ (17.9\%), supraventricular tachycardia (SVT) 6.9\% (62.5\%), ventricular tachycardia $4.3 \%$ (69.2\%). Treatments were judged optimal for primary WCT in $84.8 \%$ and suboptimal for inappropriate drug $4.4 \%$ and failure to reduce HR $<1206.3 \%$. Treatments were optimal for secondary WCT in $88.8 \%$ and suboptimal for not treating the underlying cause $6.2 \%$ and failure to reduce $\mathrm{HR}<1206.2 \%$.

Conclusion: We found that WCT cases in the ED were evenly split between primary arrhythmias and tachycardia secondary to medical issues. ED physicians interpreted the ECG correctly versus the cardiology read in $3 / 4$ of cases but under-called atrial flutter and SVT. They accurately identified the etiology of the WCT and implemented appropriate care in most cases but sometimes failed to adequately control HR or to treat the underlying condition. These findings suggest that there are opportunities to improve care of WCT in the ED. Keywords: Arrhythmia, Tachycardia

P052

\section{Prone manual and mechanical chest compression optimization} for the COVID-19 pandemic

T. Loch, BScN, D. ODochartaigh, BN, MSc, C. Picard, CD, BScN, P. Brindley, MD, M. Douma, MN

University of Calgary, Calgary, $\mathrm{AB}$

Introduction: Caring for patients in the prone position is becoming more common because of the COVID19 pandemic. Prone positioning is being used for both sedated critically ill patients and awake spontaneously breathing patients. This is resulting in more cardiac arrests in the prone position, posing a new challenge for clinicians. Resuscitation guidelines for prone patients have even been developed in response. This project aims to evaluate and optimize manual and mechanical compressions for prone cardiac arrest care.

Methods: Our study has two parts, one evaluating a prone resuscitation board and a second evaluating a mechanical compression device adapted for prone resuscitation. A CPR feedback manikin was used as a simulated prone COVID19 patient. Chest compression quality was evaluated using the quality metrics of rate, depth and release. In part one, 30 nurses performed chest compressions for 2 min atop an emergency department stretcher with and without a prone resuscitation board. The prone resuscitation board was designed with a sternal elevation to focus compressions on the sternum. In part two of our study, we adapted a mechanical compression device so its backboard could be placed under the patient's sternum and provide sternal counter-pressure while the device compressed the patient's thoracic spine 6600 times. Descriptive statistics were used to analyze data, comparisons of proportions were made using $\mathrm{Z}$ tests.

Results: Use of a prone resuscitation board improved the proportion of manual compressions achieving target depth by $46 \%$ (95\% CI $41-$ $51 \%)(\mathrm{p}<0.001)$. Participant feedback suggested that achieved target depth was less fatiguing with the prone resuscitation board compared to compressing atop a stretcher mattress. Adaptations made to the mechanical compression device improved the proportion of compressions achieving target depth from 0 to $100 \%$ over 6600 compressions. The device achieved $100 \%$ rate, release and depth target.

Conclusion: The use of a prone resuscitation board improved chest compression quality in this preclinical evaluation. Our simple and low-cost adaptation to a common mechanical compression device allowed it to perform high quality compressions on a simulated patient in the prone position, though clinical confirmation is required. Keywords: Cardiac arrest, Cardiopulmonary resuscitation, COVID19

\section{P053}

Understanding the emergency physician liaison (leader) role: a realist evaluation

T. Loch, BScN, Z. Polsky, BSc, MD, J. Elzinga, MD, E. Lang, MD, C. Patocka, MD, MHPE

University of Calgary, Calgary, $\mathrm{AB}$

Introduction: As overcrowding continues to challenge healthcare delivery in Canada, Emergency Departments (EDs) across the country 
have introduced novel emergency physician roles in hopes of providing physician leadership and coordinating patient care. In 2019, Calgary piloted the Emergency Physician Lead (EPL) role which designated a senior physician to take on managerial tasks such as expediting patient work ups, bed management and addressing challenging patient dispositions. Although similar roles have been evaluated in the literature, there remains a lack of understanding of 'how' these roles actually work to address overcrowding and whether they have other impacts. The aim of this preliminary study was to begin to understand some of the key outcomes of a physician-oriented throughput initiative, and to identify the contexts and mechanisms that either impede or enhance its efficacy.

Methods: Realist inquiry, with its focus on mechanisms acting in contexts to produce outcomes, is a unique methodology through which to understand 'how' and 'why' initiatives such as the EPL may or may not work. This realist evaluation sought to explore the impact of the 3-month pilot EPL initiative at the Foothills Medical Centre, a tertiary academic ED which sees $>75,000$ visits per year. Between December 2019 and January 2020 we conducted semi-structured interviews of key stakeholders in the initiative. Interviews were transcribed and analyzed using thematic and template analysis techniques specifically aimed at identifying context, mechanism and outcome configurations.

Results: 13 semi-structured interviews were conducted with emergency physicians and emergency registered nurses. The EPL role appeared to have impacts on ED throughput, patient safety and team dynamics. Importantly, we identified how various contexts and mechanisms could either promote (for example, initiating time sensitive treatments), or impede (for example, nursing unable to complete proposed treatments) these outcomes, and ultimately impact ED department throughput, patient safety and team dynamics.

Conclusion: Emergency Department physician-liaison interventions demonstrate that such a role has perceived impacts on throughput, patient safety, and team dynamics. Future evaluations and implementations of physician lead initiatives should consider these outcomes carefully in their monitoring and measurement of impact.Learnings from this realist study may be particularly helpful in informing future physician roles aimed at addressing ED overcrowding. Keywords: Patient safety, Realist evaluation, Throughput

\section{P054}

Surviving strangulation: evaluation of non-fatal strangulation in patients presenting to a tertiary care sexual assault and partner abuse care program

Z. MacDonald, MD, D. Eagles, MD, MSc, K. Yadav, MD, MSc, K. Muldoon, MPH, PhD, K. Sampsel, MD, MSc

Department of Emergency Medicine, University of Ottawa, Ottawa, ON

Introduction: Non-fatal strangulation (NFS) is a dangerous mechanism of injury among survivors of intimate partner violence (IPV) and sexual assault (SA), with inadequate evidence to guide imaging investigations in the Emergency Department (ED). The primary objective is to identify the proportion of IPV and SA where NFS occurs, and to describe the sequelae of NFS injuries. A secondary objective is to investigate the rate and yield of diagnostic imaging.

Methods: We conducted a health records review of patients treated at the Sexual Assault and Partner Abuse Care Program (SAPACP) and/ or Trauma Program at a tertiary level hospital (January 2015-December 2018). Eligible patients: $\geq 16$ years, seen by the SAPACP or trauma team for IPV/SA, with NFS identified. Data was abstracted from the standardized assessment completed by the SAPACP nurse. Descriptive statistics were used.
Results: We identified 209 eligible cases of NFS, amongst 1791 patient presentations to the SAPACP $(11.7 \%)$. Patients had a median age of 27 years, and $97.6 \%$ were female. Computed tomography (CT) of the head was obtained in $22.5 \%$, and CT angiography (CTA) of the head/neck in $6.2 \%$ of cases. Eleven significant injuries were identified. Two cases of vascular abnormalities: internal carotid artery (ICA) indentation with possible intramural hematoma and possible ICA dissection. Other injuries included bilateral subdural hematomas on repeat imaging and a depressed skull fracture. Six patients had nasal fractures, all managed conservatively.

Conclusion: We found over $10 \%$ prevalence of NFS in survivors of IPV/SA. There was a low rate of significant injury related to NFS, with severe injuries resulting from concomitant trauma. Yield of CTA in this cohort was low. Further work to guide imaging in this population may be of benefit.

Keywords: Intimate partner violence, Non-fatal strangulation, Sexual assault

\section{P055}

Provision of non-pharmacologic analgesia to children by prehospital providers in southwestern Ontario: a crosssectional study

C. Mace, BSc, N. Poonai, MD, MSc, B. Vujcic, MSc, K. Burke, BSc, J. Hamilton, BSc, J. Teefy, MD

Western University, London, ON

Introduction: There is abundant evidence that in children, management of pain by prehospital providers is suboptimal. However, provision of non-pharmacologic analgesia has not been described. Most paediatric calls are performed by primary care paramedics (PCPs) who are unable to administer pharmacologic analgesia to children but can administer non-pharmacologic therapies. We sought to describe the proportion of children provided non-pharmacologic analgesia by prehospital providers.

Methods: We reviewed all ambulance call reports (ACR) of children age $0-17$ years with an acutely painful condition (headache, abdominal pain, possible fracture, head/ears/eyes/nose/throat pain, back pain, and unclassified pain) who were transported to the Children's Hospital, London Health Sciences Centre from 2017 to 2019. We excluded ACRs lacking data pertaining to the primary outcome. Data collection was recorded by two blinded assessors using a study specific ExcelTM sheet. The primary outcome was the proportion of children offered non-pharmacologic analgesia. Using a complete case analysis, we performed a hierarchical stepwise logistic regression on the primary outcome using covariates defined a priori: age, sex, visible deformity, type of crew, complaint, documented pain assessment, call time, and prior analgesia.

Results: All 11,084 ACRs from January 1, 2017 to December 31, 2019 were reviewed. The sample included 5887/11,084 (53.1\%) males, ranging from 1 month to 17 years, with a mean (SD) age of 10.5 (5.6) years. Calls involved mainly PCPs [8576/11,084 (77.4\%)]. Non-trauma related musculoskeletal injuries were most common, comprising 2743/11,084 (24.7\%) of calls. Pain scores were documented in $6947 / 11,084(62.7 \%)$ of calls. The verbal numeric rating scale (0-10) was used in 5022/6947 (72.3\%) calls, with a mean (SD) score of 6 (moderate pain). Non-pharmacologic analgesia was provided in 2926/11,084 (26.4\%) of calls, most commonly splint (1115/ $2926,38.1 \%)$ and ice $(931 / 2926,31.8 \%)$. Pharmacologic analgesia was provided in $458 / 11,084(4.1 \%)$ of calls. In the multivariable analysis model, the only significant predictor of non-pharmacologic analgesia was age $<7$ years (OR 1.4; $95 \%$ CI 1.1, 1.8; $\mathrm{p}=0.005$ ). Conclusion: The provision of non-pharmacologic analgesia to children in Southwestern Ontario by prehospital providers is suboptimal 
despite moderate to severe levels of pain. There is a clear need for education surrounding pain assessment and non-pharmacologic analgesic options in children among prehospital providers.

Keywords: Paediatrics, Pain, Prehospital

\section{P056}

\section{Assessing the impact of the implementation of an advanced care paramedic program in the greater Saint John area in patients with out of hospital cardiac arrest}

M. McGraw, BSc, MD, MSc, P. Atkinson, BSc, MB, J. Fraser, RNBN, T. Pishe, MD, J. Mekwan, MBBS(Lond), K. Chandra, BSc, $\mathrm{MD}, \mathrm{MSc}$

Saint John Regional Hospital Department of Emergency Medicine, Saint John, NB

Introduction: Deployment of advanced care paramedics (ACPs) has enabled enhanced prehospital care of patients with out of hospital cardiac arrest (OHCA) reducing the delay in epinephrine administration and advanced airway management. The objective of this study was to determine the impact of the introduction of a regional ACP program on rates of return of spontaneous circulation (ROSC), survival to hospital admission (SHA), and discharge (SHD) in OHCA patients transported to hospital.

Methods: We performed a health records review of patients with OHCA transported to a tertiary Canadian Hospital from January 2010 to December 2014 for pre-ACP implementation, and January 2016 to March 2019 for post-ACP implementation. All patients presenting to the ED with a diagnosis of cardiac arrest were included in the data collection. Patients under 18 years, or who arrested in the emergency department, suffered a traumatic arrest or suffered a cardiac arrest after an overdose were excluded. Demographic data and primary outcomes were collected from the prehospital ambulance and hospital medical record. Outcomes from each group were analyzed using ChiSquare tests to determine significance.

Results: 228 of 264 potential patients (receiving only basic life support) were included in the pre-ACP implementation group, with 162 of 228 patients in the post-ACP implementation group. Groups were similar for age (64.3 vs 64.7 years) gender ( $68 \%$ vs $67 \%$ male) and mean time to EMS arrival (7.1 vs $7.0 \mathrm{~min}$ ). Rates of ROSC $\left(41.3 \%\right.$ vs $\left.31.6 \%, X^{2} 3.8372, p=0.05\right)$; SHA $\left(24.7 \%\right.$ vs $14.9 \%, X^{2}$ $6.920, \mathrm{p}=0.015)$ and SHD $\left(11.1 \%\right.$ vs $\left.3.1 \%, \mathrm{X}^{2} 14.1963, \mathrm{p}<0.001\right)$ were higher in the post-ACP implementation group.

Conclusion: We report improvements in rates of ROSC, SHA and SHD in OHCA patients presenting to the ED following the introduction of an ACP program to a region in New Brunswick, Canada. Keywords: Advanced cardiovascular life support, Advanced care paramedics, Cardiac arrest

\section{P057}

Emergency physician risk assessment practices prior to prescribing opioids

M. McKinney, MD, I. Stiell, MD, MSc, M. Kisilewicz, MD

University of Ottawa, Department of Emergency Medicine, Ottawa, ON

Introduction: Safer opioid prescribing remains a crucial issue for emergency physicians. Prescribing guidelines and policy statements recommend routine risk assessments for likelihood of current or future opioid use disorder prior to prescribing opioids. We sought to establish the practice patterns of Canadian emergency physicians in assessing this risk prior to prescribing opioids from the ED: are physicians using published risk assessment tools, identifying risk factors, relying on gestalt, or perhaps not making these assessments at all?

Methods: We surveyed CAEP members across Canada about their practice patterns of risk assessment prior to prescribing opioids from the ED. We also asked about local opioid prescribing policies, use of electronic records to aid decision making, familiarity with the literature, and which clinical risk factors they find most important in making their assessments. The survey was 19 questions. We completed a local pilot test prior to distribution using a modified Dillman approach with 3 mailings. Data were analyzed through descriptive statistics and thematic analysis.

Results: We had 312 responses from 1532 physicians receiving survey emails. $59.8 \%$ of respondents report usually or always assessing for risk. Physicians mainly rely on gestalt (80.3\%), review of medical history $(83.1 \%)$ and medication records $(75.6 \%)$, and targeted histories based on risk factors from their experience $(57.6 \%)$ or in the literature $(55.6 \%)$. Contacting primary prescribers is uncommon $(16.3 \%)$. Very few routinely use opioid prescribing risk assessment tools (6.4\%), have local opioid prescribing policies $(27 \%)$, or make use of electronic medical record functions to assist risk stratifying $(2.4 \%)$. Few physicians report prior formal training through modules or courses on opioid prescribing $(31.1 \%)$. The most common features emergency physicians find important in their risk assessments are medication history, nature of the pain, and substance use history.

Conclusion: Many Canadian emergency physicians make risk assessments based on gestalt rather than explicitly identifying literature-based risk factors or using risk assessment tools. This conflicts with guidelines that call for routine comprehensive assessment. Further efforts should be directed toward education in optimizing risk assessment; and toward system-level initiatives such as clear local prescribing policies, electronic-systems functionality, and developing assessment tools that will be used by emergency physicians.

Keywords: Opioid, Prescribing, Risk

\section{P058}

First Nations status and emergency department triage scores in the Canadian province of Alberta: a retrospective cohort study using linked administrative data

P. McLane, PhD, C. Barnabe, MD, MSc, L. Mackey, BA, B. Holroyd, MBA, MD, K. Rittenbach, PhD, K. Janiver, BA, L. Bill, BScN, R. Rosychuk, PhD

Alberta Health Services Emergency Strategic Clinical Network/ University of Alberta, Edmonton, AB

Introduction: This study examines emergency department triage scores of First Nations and non-First Nations patients in Alberta. Our objective was to identify significant factors related to acuity of triage scores for First Nations compared to non-First Nations patients.

Methods: We conducted a population-based retrospective cohort study for the period April 2012 to March 2017 using administrative data. Our binary outcome was acute triage score (Resuscitation or Emergent) or less acute triage score. Multivariable multi-level logistic mixed-effects regression models were developed. Subgroup analyses considered specific diagnoses and disease categories (defined using 3 M Episode Disease Categories).

Results: The dataset contains 10,826,591 emergency care visits including 1,281,663 visits by First Nations patients. Compared to non-First Nations patients, First Nations patients had lower odds of acute triage if they had a comorbidity [odds ratio 1.24 (95\% confidence interval (CI) $1.21,1.27$ ) for First Nations vs. 1.51 (CI 1.50, 1.52) for non-First Nations], were older [1.01 (CI 1.01, 1.02) vs. 1.02 
(CI 1.02, 1.02), per 10 years of age], or lived in a lower income area [0.90 (CI 0.89, 0.92) vs. 1.03 (CI 1.02, 1.03)]. Using presentations to large community hospitals as a baseline, First Nations patients had lower odds of acute triage when they presented to mixed tertiary sites [6.24 (CI 6.07, 6.41) vs. 6.72 (CI 6.66, 6.79)], regional hospitals [3.87 (CI $3.77,3.97$ ) vs. 4.25 (CI 4.21, 4.28)] medium community hospitals $[1.04$ (CI 1.01, 1.08) vs 1.17 (CI 1.16, 1.19)] and small community hospitals $[0.46$ (CI $0.44,0.49$ ) vs. 0.72 (CI 0.71, 0.73)]. First Nations patients had higher odds of acute triage when presenting to tertiary pediatric hospitals [6.75 (CI 6.50, 7.01) vs. 4.62 (CI 4.57, 4.68)] and when arriving by ambulance $[3.54$ (CI $3.47,3.61$ ) vs. 2.55 (CI 2.54, 2.57)]. Differences in odds ratios reported above are significant at the $\mathrm{p}<0.05$ level. Male patient sex did not have a significantly different impact on triage scores for First Nations compared to non-First Nations patients [1.11 (CI 1.09, 1.13) vs. 1.12 (CI 1.11, 1.12)]. Findings related to area income and facility type were robust to difference in diseases categories, while findings related to facility type were also robust to differences in specific diagnoses.

Conclusion: First Nations status relates to triage scores and interacts with other factors. First Nations patients had lower odds of acute triage in smaller hospitals.

Keywords: Equity, First Nations, Triage

\section{P059}

Do patients respond to posted ED wait times: evidence from the implementation of a wait time publication system in Hamilton, Canada

S. Mondoux, MD, MSc, S. Strobel, MD, MA(Econ), K. Ren, MD, A. Dragoman, MD, C. Pettit, BA, A. Stancati, BA, D. Kallergis, M. Smith, MBA, A. Pardhan, MBA, MD, K. Sidhu, MD, R. Vadera, $\mathrm{MD}, \mathrm{G}$. Rutledge, MD

McMaster, Hamilton, ON

Introduction: Prolonged Emergency Department (ED) wait times are a focus of improvement for many international health systems. Many solutions have been proposed to address this problem and reduce the time required for patients to be seen by ED physicians. Among these are publicly available websites which display ED wait times. We describe the effect of a novel city-wide ED wait times website on patient volume distributions throughout the city.

Methods: We compared data from before and after implementation. A total of 7 clinical sites were included: 3 adult tertiary care EDs, 1 tertiary pediatric hospital, 1 community hospital and 2 urgent care centers. These clinical sites serve a population of about 750,000 people. The posted wait times for each site as well as the total number of new patient arrivals were obtained for each 15-min interval within this timeframe for a total of 20,912 observations. For each ED site, the effect of the posted wait time was measured by assessing the total number of patient arrivals in the subsequent hour. The effect of wait times of one site on itself as well as on all others was measured and described.

Results: The wait times site resulted in reduced predicted wait times across all participating sites in this pre-post analysis. All sites resulted in a lower post-intervention patient arrival rate with the exception of the site posting the shortest PIA times throughout the trial. Comparing changes across all sites relative to the mean, a reduction in $50 \mathrm{~min}$ resulted in a $2-3 \%$ volume increase in the next hour while an increase in $50 \mathrm{~min}$ from the mean resulted in a $10 \%$ reduction in volumes at the same site. Significant pattern differences existed across the sites with the highest volume variability at the urgent care centers. Relationships existed between specific clinical sites and increased wait times although an increase or decrease in wait times at a single site did not yield system-wide increases or decreases. Different utilizations behaviours were observed with increased wait times between urgent care and specialist sites.

Conclusion: ED wait time posting for all clinical sites in a large geographical area appears to reduce whole system wait times and redistribute some patients to sites with lower wait times.

Keywords: Patient flow, Quality improvement and patient safety, Wait times

\section{P060}

A quality improvement project to ensure safe airway management in the emergency department in the time of COVID19

D. Morden, BSc, MD, E. Hatam, MD, S. Mal, MD, B. Vujcic, BSc, J. Pace, MD

Western University, London, ON

Background: In March 2020 the COVID-19 pandemic forced emergency departments across the world to rapidly adapt to the impending threat that the severe acute respiratory syndrome coronavirus- 2 posed to both patients and health care providers. Respiratory support and airway management was of paramount concern given the anticipated influx of COVID-19 pneumonia patients that often developed severely deranged respiratory physiology, posing a risk to themselves and those caring for them. Our institution implemented hospital wide protocols, including the development of multidisciplinary intubation teams. Introducing new protocols and care teams can have unanticipated outcomes, particularly when implemented for patient populations outside the group for which they were developed. We sought to observe the influence these organizational changes had on airway management outcomes in the emergency room. Our aim was to observe the impact that the organizational COVID-19 airway management protocols had on patient care during the study period.

Aim statement: Utilizing the LHSC ER airway registry we compared airway outcomes during a 3-month period of COVID-19 protocols to outcomes preceding these organizational changes. Outcomes measured were predefined key performance indicators (KPIs): maintenance of systolic blood pressure greater than $90 \mathrm{mmHg}$, oxygen saturation greater than $90 \%$, endotracheal tube delivery in less than $90 \mathrm{~s}$ from medication administration, and first pass success.

Measures and design: 128/193 ED intubations from July 2018March 2020 met our KPIs, as compared to 20/35 intubations during the COVID19-protocol period (66\% vs 57\%). Indications for intubation were similar pre and post-COVID-19 protocols; altered mental status (21\% vs $28.6 \%)$, cardiac arrest (17.7\% vs $20 \%)$ and overdose $(15.2 \%$ vs $8.6 \%)$ were the top 3 indications. First-pass success rate decreased by $8.9 \%$ (66\% vs $57 \%)$.

Evaluation/results: There are multiple factors that could account for this drop in "successful" airways (those meeting KPIs). Limitations in pre-oxygenation, using a less familiar intubating device, and delays in connecting to the ventilator are all potential contributing factors. These data serve as an important reminder to be vigilant for the secondary harms of altered protocols in the era of the COVID-19 pandemic, reinforcing the need for ongoing training and education. Locally, this data has been used at inter-disciplinary QI rounds to address systems issues that could be negatively impacting airway management performance indicators.

Keywords: COVID-19, Intubation, Quality improvement and patient safety

\section{P061}

Exploring communication between consultants and referring physicians: a qualitative study 
A. Muftah, BSc, P. Kapur, MD, MSc, J. Stempien, BSc, MD, T. Oyedokun, MBChB, MMed

\section{University of Saskatchewan, Saskatoon, SK}

Introduction: Effective communication between physicians is a core competency in medicine. However, requests from referring physicians for advice from consultants are often subject to misunderstanding and professional incivility. While existing literature focuses on the role of referring physicians, little is known on how consultants can improve the structure and substance of the advice they provide. Third-party perspectives on communication between physicians offer a unique vantage point to improve such interactions. Until recently, phone conversations involving physicians from Saskatoon were mediated by trained telecommunicators through the Acute Care Access Line (ACAL). This study explored the experiences of ACAL operators to: (1) identify the effective and ineffective components of communication between physicians; (2) recognize the factors which trigger and sustain tensions during physician-to-physician communication; (3) generate concrete recommendations to aid consultants in providing advice.

Methods: Thirteen ACAL operators were recruited to participate in individual semi-structured interviews. All interviews were conducted via phone call, audio-recorded, and transcribed verbatim. Interview transcripts were analysed using a grounded theory approach. Ethics approval was obtained from the University of Saskatchewan Biomedical Research Ethics Board.

Results: Constant comparative analysis revealed linguistic, personal, and situational features of telephone consultations as three main sources of tension. Communication breakdowns due to these tensions were implicated in delays in patient care, transport and care miscommunications, avoidance of consultants by referring physicians, and overwhelming other medical services including emergency medicine. Identified sources of tension were placed in relation to one another to develop a scheme to aid consultants in providing professional advice to referring physicians.

Conclusion: This study provides a conceptual framework for understanding tensions during physician-to-physician communication. Future process improvement efforts and educational interventions may be informed by this model to reduce perceived tension during physician-to-physician communication and increase the utility of consultations.

Keywords: Consultation, Interprofessional relations, Physician communication

\section{P062}

Improving acute care for long-term care residents: a better way to care for the frail elderly in times of medical urgency

A. Munene, BSc, PhD, MES, D. Alaazi, BSc, PhD, MPhil, MA, L. Wyer, BHSc, MSc, S. Reid, MN, E. Lang, MD CM, V. Ewa, MD, G. Cummings, MEd, PhD, BScN, E. Spackman, BA, PhD, MA, P. McLane, BA, PhD, MA, P. Faris, BSc, MSc, PhD, H. Hair, MBA, M. George, BN, EdD, J. Holroyd-Leduc, MD

University of Calgary, Calgary, $\mathrm{AB}$

Background: LTC residents undergo acute changes in health status that lead to transfers to the emergency department (ED). Many of these changes could be evaluated and managed at the long-term care (LTC) site if appropriate supports were available. Furthermore, transfer to ED can lead to iatrogenic harms and discontinuity in care. The aim of this integrated knowledge translation project is to identify changes in resident's health status sooner, improve communication between LTC and ED, and reduce unnecessary transfer to ED.
Aim statement: 38 LTC facilities in the Calgary zone are involved in implementation of a standardized LTC-to-ED care and referral pathway, known as RAAPID (Referral, Access, Advice, Placement, Information, and Destination) supported by a centralized telephone triage system, community paramedics, and INTERACT ${ }^{\circledR}$ tools focused on early identification and management of acute medical issues. A randomized stepped-wedge study design is being used to sequentially implement and evaluate this change. Implementation has been informed by the knowledge to action (KTA) cycle, with implementation strategies being adapted to the local barriers and context of each facility. Our primary outcome of interest is the change in the rate of transfers to ED from LTC facilities adjusted by residentdays. Secondary outcomes will assess impact on key quality dimensions, cost efficiency, and will include interviews with residents and focus groups with healthcare providers.

Measures and design: To date the standardized LTC-to-ED care and referral pathway has been implemented at 21 LTC facilities and will be implemented at all 38 sites by August 2021. Implementation has been supported by a centralized implementation coach, site champions, educational sessions, point of care tools and audit-and-feedback reports. Early findings from 11/38 facilities demonstrate a reduction in the four month mean (sd) number of visits to ED per 1000 resident days from 1.9 visits $(0.77)$ before to 1.6 visits (0.68) after implementation. The mean (sd) number of calls to RAAPID per 1000 resident days increased from 0.2 calls (0.53) to 0.9 calls (1.42) after implementation. The mean number of community paramedic visits to LTC facilities per month has not changed (1.8 visits (2.24) before to 1.9 visits (2.99) after implementation).

Evaluation/results: The intervention is expected to result in fewer transfers from LTC to the ED, while also achieving better outcomes for patients. The aim is to provide the right care in the right location, in a cost-efficient manner.

Keywords: Emergency department, Long-term care, Quality improvement and patient safety

\section{P063}

A novel transition to practice curriculum for $\operatorname{CCFP(EM)~}$ programs

A. Nath, MD, W. Cheung, MD, MMed, J. Leppard, MD, J. Perry, $\mathrm{MD}, \mathrm{MSc}$

Department of Emergency Medicine, University of Ottawa, Ottawa, ON

Innovation concept: The importance of a resident's ability to transition to practice is highlighted in the medical literature. We implemented a novel transition to practice curriculum incorporating four new processes to prepare CCFP(EM) residents for independent practice. These elements were: explicit sequencing of competency progression; establishment of coaching teams; establishment of independent shifts; and implementation of a transition to practice seminar series.

Methods: The utility of these curricular components as perceived by residents was assessed over 3-year following implementation. Electronic surveys were sent to all graduating CCFP(EM) residents in their last week of residency with a follow-up email 3 days after. We used descriptive statistics (percentages) assessing resident satisfaction and their perceived readiness for independent practice.

Curriculum, tool, or material: We developed a "Progression of competence shift card" based on a prior peer-reviewed framework. A list of competencies based on early, middle and late stages of training were developed using a modified Delphi method. Residents discussed these at the start of each shift allowing for a shared mental model of expectations with their supervisor. We utilized "clinical coaching 
teams' that comprised of 3 emergency physicians. Residents worked $75 \%$ of their shifts with their team with the intent of establishing an educational alliance. This allowed fOR comfort with practice style; continuity of teaching, learning and assessment; and mentoring throughout the year. Residents were assigned to work independently in a low acuity care area in their last stage of training to build autonomy and work on patient flow. A supervising physician met the resident at the end of the shift to review cases and discuss nuances of patient care, billing, charting, medico-legal aspects, and flow. A series of in-person seminars were designed to incorporate previously identified transition-to-practice content including: billing; personal finance management; wellness; medico-legal pitfalls; flow; charting; and contract negotiations.

Conclusion: All residents surveyed found independent shifts useful, and liked the idea of clinical coaching teams. $88.2 \%$ residents reported feeling ready for independent practice at the end of training. We described a novel transition to practice curriculum for a 1-year training program. Residents responded positively to this curriculum. This framework may be used by other programs to improve residents' readiness for independent practice.

Keywords: $\mathrm{CCFP}(\mathrm{EM})$, Innovations in EM education, Transition to practice

\section{P064}

Validation of ICD10 diagnostic codes for acute aortic syndrome and development of a diagnostic algorithm for identification of cases using administrative database

R. Ohle, MBChB, MSc, MA, D. Savage, MD, PhD, B. Paige, MD, S. McIsaac, MBChB, MEd

\section{Health Science North, Sudbury, ON}

Introduction: Acute aortic syndrome is a rare time sensitive aortic catastrophe that caries a high mortality if missed. Currently we miss $>20 \%$ of cases and our mortality is twice that of other developed countries. Give its incidence it is a difficult condition to prospectively study. Administrative database hold great opportunity for assessing outcomes for large implementation studies to improve care for this deadly condition. It is unknown if the ICD 10 code for acute aortic syndrome is sufficiently accurate to be used as an outcome for a large multi centre study. Our primary objective was to assess the diagnostic accuracy of ICD 10 codes for the identification of acute aortic syndrome. Our secondary objective was to derive a diagnostic algorithm for the identification of cases of acute aortic syndrome for use with administrative database.

Methods: We performed a retrospective cohort study at an academic tertiary care hospital from 2013 to 2018. Two independent reviewers extracted data from cases identified by emergency department or hospital discharge code of ICD 10 I71. Confirmed cases of acute aortic syndrome were defined by; Radiology report on computed tomography of an acute aortic dissection, intramural hematoma or penetrating atherosclerotic ulcer, Intra operative diagnosis of AAS, post mortem diagnosis of AAS. Sensitivity and positive predictive value were calculated together with $95 \%$ confidence interval. Logistic regression was performed to select for variables that predict the confirmed diagnosis of AAS.

Results: 92 patients [mean age (SD) 62.4 (16) years, 36 (39\%) female] were found with an ICD 10 I71.0. True incident diagnosis of acute aortic syndrome was confirmed in $46(50 \%)$ patients. The positive predictive value of ICD 10 I71.0 was 50\% (95\% CI 42.8 57.1). Variables significantly associated with true cases of AAS were admitted [ $\mathrm{n}=66(72.5 \%) \mathrm{p}<0.008]$, AAS as main diagnosis $[\mathrm{n}=46$ $(50 \%) \mathrm{P}<0.0001]$, length of stay $<2$ days and died $[\mathrm{n}=6(6.5 \%)$ $\mathrm{p}<0.001]$. Length of stay/died $(\mathrm{P}<0.049)$ and AAS as main diagnosis $(\mathrm{p}<0.001)$ were independent associated with a true diagnosis (c statistic 0.9 ). The positive predictive value of a combination of these variables to identify cases was $93 \%$ (95\% CI 85.1-97.3\%). Conclusion: We have demonstrated that acute aortic syndrome ICD 10 code I71 is not sufficiently accurate to be used to identify cases. We have derived a diagnostic algorithm with excellent accuracy that if validated will allow us to accurately identify cases of AAS within health administrative data.

Keywords: Acute aortic syndrome, Administrative data

\section{P065}

Frequency of blood transfusion in patients presenting to the emergency department with iron deficiency anemia

E. Oura, BSc, MD, Z. Solh, MD, MSc, B. Vujcic, BSc, J. Kim, MD, MSc

Western University, London, ON

Introduction: Iron deficiency anemia (IDA) is a common presentation to the emergency department (ED). Blood transfusion is a readily available and effective treatment but poses several risks to patients, particularly women of childbearing age and elderly patients. Intravenous (IV) iron is an alternative to blood transfusion and may be a more appropriate intervention in many cases; however, availability, cost, provider familiarity and feasibility limit its use in many EDs. Consistent practice guidelines for management of IDA in the ED are lacking. Understanding local management of IDA is important for ensuring safe and appropriate management and for informing future practice.

Methods: We conducted a retrospective cohort study between January 1st, 2018 and December 31st, 2018. We aimed to describe the patient population presenting with IDA and current practice patterns, particularly the frequency of blood transfusion. We selected the study population based on age $\geq 15$ years, initial hemoglobin $<90 \mathrm{~g} / \mathrm{L}$, initial MCV $<80 \mathrm{fL}$ and the administration of blood transfusion and/ or IV iron in the ED.

Results: A total of 209 patients were included. The mean hemoglobin was $68.7 \mathrm{~g} / \mathrm{L}$ and mean $\mathrm{MCV}$ was $69.8 \mathrm{fL}$. In total, $120(57.4 \%)$ patients received a blood transfusion, while $4(1.9 \%)$ received IV iron. In women of child-bearing age (15-45 years), 26 patients $(38.8 \%)$ received a blood transfusion and $2(3.0 \%)$ received IV iron. The length of stay in the ED was significantly longer for patients who were transfused compared to those who were not (11.0 vs $7.1 \mathrm{~h} ; \mathrm{p}<0.001)$. In patients who were transfused versus those who were not transfused, triage vital signs were similar between groups, however there was a higher rate of reported fatigue (53.3 vs $43.8 \%)$, shortness of breath ( 50.8 vs $23.6 \%)$, light-headedness ( 32.5 vs $22.5 \%$ ) and chest pain (15.8 vs $7.8 \%$ ) on initial assessment in those requiring transfusion.

Conclusion: The results of this study demonstrate that the current frequency of blood transfusion in patients presenting to the ED with IDA is much higher than the frequency of IV iron infusion. Additionally, patients who are transfused blood for this indication spend more time in the ED. Based on these results, the current management of IDA could be optimized as recent literature recommends IV iron as a safe, efficient and effective alternative to transfusion. Creation of clear guidelines and quality improvement measures are required in order to optimize management of this common ED presentation.

Keywords: Anemia, Emergency department, Transfusion

\section{P066}

Establishing a shared mental model during COVID-19 using simulation and real-time interdisciplinary knowledge translation 
C. Parpia, BHSc, A. Dharamsi, BSc, MD

University of Toronto, Toronto, ON

Innovation concept: During the COVID-19 pandemic, guidelines and protocols are being developed rapidly; however there is a gap between protocol development, and dissemination to the interdisciplinary healthcare provider (HCP) team. Simulation is a key tool to address this gap, via education, team-based training, and fostering a shared mental model (SMM). SMMs in the emergency department (ED) enable members of high performing teams to predict their teammates' resource needs and next steps, especially in situations where communication is difficult. The key innovation was the combination of in-situ simulation with parallel, real-time, knowledge translation (KT) to an interdisciplinary team via infographics. The primary objective was to design infographics that could facilitate the rapid establishment of an SMM amongst interdisciplinary resuscitation teams in the ED.

Methods: Five simulation sessions were performed at an academic, tertiary care centre in Toronto, Ontario. In parallel with these sessions, two infographics were created, updated iteratively, and disseminated to the interdisciplinary ED team over the course of 6 weeks from January to March 2020. Infographics evolved according to real-time feedback from the simulation sessions, the development of institutional COVID-19 guidelines, and the scientific understanding of COVID-19.

Curriculum, tool, or material: Two infographics were iteratively developed and designed for use in real-time resuscitations through the use of specific design elements (e.g. systematic organization, hierarchical consistency, and emphasis of information). These infographics aided in establishing an SMM by serving as a comprehensive pointof-care reference of key resuscitative principles, treatment considerations, and a systematic approach to collaborative teamwork. The infographics expanded the reach of the COVID-19 in-situ simulation findings well beyond the original 56 simulation participants through distribution via social media, print, email, and workshops.

Conclusion: The combination of interdisciplinary in-situ simulation with parallel KT enabled the rapid establishment of an SMM within the institution's ED. Implementation of infographics subjectively augmented coordination within ED teams, and improved patient care. Although qualitative feedback has been positive, future research will involve objectively evaluating the infographics to inform future forms of KT, using frameworks like the Kirkpatrick Program Evaluation model.

Keywords: Innovations in EM education, Knowledge translation, Shared mental model

\section{P067}

Developing the equity, diversity, advocacy, and cultural safety (EDACS) curriculum for cultural change in the emergency department

S. Qayyum, BSc, J. Hulme, BHSc, MDCM, MPH, K. Hayman, MD, MPH, E. Dell, MD, MPH, E. Fremes, MPH

University of Toronto, Toronto, ON

Innovation concept: Canadian physicians require knowledge beyond the traditional medical repertoire to appropriately respond to patient and community needs. Members of marginalized communities, and professional and accreditation bodies are calling for improved training. We developed the equity, diversity, advocacy, and cultural safety (EDACS) curriculum to teach content and skills required to advocate for patients and policy change. The goal is for residents to act on healthcare inequities and not just be aware of them. This curriculum launched in 2019 for UofT EM residents. It consists of $8 \mathrm{~h}$-long sessions and a walking tour of sites accessed by individuals living in poverty. We chose session themes via a resident survey, which identified perceived knowledge gaps. In order to support the ongoing implementation of this curriculum, our team is now developing primers for each of the session themes. These will provide trainees with a readily accessible resource for key information and actionable items.

Methods: Throughout the summer of 2020, we conducted an extensive search for each EDACS session theme, including the following populations: 2SLGBTQI+, homeless, Indigenous, Black, and newcomers. Our search covered academic articles, podcasts, media pieces, online modules, and personal narratives. Each resulting primer has 5 priority areas: background, health inequities, landscape of healthcare in the ED, tips to improve care, and opportunities for local ED policy change and advocacy work.

Curriculum, tool, or material: The primers summarize options for improving care for each population theme, focusing on actionable items for residents. After our team's revision, primers will undergo further review by: (A) specific ED content expert, (B) healthcare content expert, and (C) individuals with lived experience and/or community organizations that directly serve them.

Conclusion: This is the first curriculum of its kind in a Canadian EM training program. The material collated in each primer can also be used to create additional educational resources, including videos, infographics, and/or podcasts for EM providers. Our goal is to help transform the culture of the ED, beginning with how certain sociallyexcluded populations are viewed, to improve their care and health outcomes. After sharing our findings with the resident group and local EM residency training committee, the end-goal is to have the curricular sessions and corresponding primers implemented into all Canadian EM residency programs.

Keywords: Cultural safety, Equity advocacy, Innovations in EM education

\section{P068}

CRACKCast: a model for best practices in free open access medical education

D. Radomske, BN, MD, B. Thoma, MD, MSc, MA, O. Scheirer, BSc, MD, R. Carey, BSc, MD

University of Saskatchewan, Saskatoon, SK

Innovation concept: The use of open educational resources such as podcasts to educate medical students and residents is commonplace and they are increasingly being integrated into formal curricula. However, these resources are rarely organized in keeping with best practices for learning and seldom follow a formal curriculum. We sought to remedy this by creating a suite of open educational resources that follow a curriculum and align with best practices.

Methods: CRACKCast is hosted on the CanadiEM website (https://canadiem.org) and consists of a comprehensive curriculum that tracks the chapters of the Rosen's Emergency Medicine textbook. It employs multiple modalities (podcasts, blog posts, and online flashcard decks) to support evidence-based learning practices such as spaced repetition, interleaving, and test-enhanced learning.

Curriculum, tool, or material: The full CRACKcast curriculum consists of 211 podcast episodes and blog posts that correspond with the chapters of Rosen's Emergency Medicine. Flashcards have been created for $85 \%$ of the episodes. The resources follow a question-andanswer format to facilitate test-enhanced learning and are designed to be used consecutively (e.g. read the chapter, listen to the podcast, review the show notes, and quiz with the flashcards) to support spaced repetition and interleaving. 
Conclusion: Podcast episodes have been downloaded by listeners from 119 countries more than 1,186,000 times since its creation. The flashcards have been downloaded over 6486 times. Additional research is required to determine CRACKCast's efficacy as an educational tool, but we believe our innovation demonstrates the integration of best practices in this novel learning modality and has the potential to inform the development of similar curricula in other fields. Keywords: FOAM, Innovations in EM education, Podcast

\section{P069}

Comparing the written notes of resident and attending physicians for patients presenting with abdominal pain to a Canadian academic emergency department: a pilot study

A. Rajaram, MD, MMI, S. Van Heer, BSc, S. Srivastava, BSc, MD, MSc, B. Wolfrom, BSc, MD, S. Douglas, BSc, MD, MSc

\section{Queen's University, Kingston, ON}

Introduction: Documentation is a critical part of the physician-patient encounter yet issues remain in accuracy and completeness with implications for clinical decision-making and quality of care. Research has demonstrated incomplete electronic medical record (EMR) documentation of history and physical examination findings for common emergency department (ED) presentations. However, little is known about the completeness of the assessment and plan (A\&P) section. We assessed the completeness of the A\&P of ED chart notes and explored potential differences between resident and attending physicians to identify targeted learning opportunities.

Methods: We conducted a single-centre, retrospective cross-sectional study involving an audit and abstraction of data from the EMR. All patients who presented with abdominal pain between January 1 and 31,2016 and were assessed by an attending or resident physician were eligible for inclusion $(n=544)$. One hundred patient charts were randomly selected. One attending emergency physician-educator assessed the completeness of the A\&P using an adapted version of the QNOTE, a validated instrument for assessing electronic note quality. The maximum possible score was 16 . Differences in scores among attending physicians and trainees were compared. The project received local research ethics board approval prior to initiation.

Results: $50 \%(n=50)$ of charts were authored by residents and clinical fellows and 50\% $(\mathrm{n}=50)$ were authored by staff physicians. Trainees represented a number of programs, including family medicine $(n=10)$, emergency medicine $(n=25)$, anesthesiology $(n=6)$, internal medicine $(n=3)$, obstetrics and gynecology $(n=2)$, orthopedic surgery $(\mathrm{n}=2)$, and psychiatry $(\mathrm{n}=1)$. Total A\&P completeness scores ranged from 0 to 16 with an average of 13.05. Resident scores ranged from 6 to 16 with an average of 13.63. Attending physician completeness scores ranged from 0 to 16 with an average of 12.5 .

Conclusion: We conducted a pilot study exploring the completeness of the A\&P section of ED notes by resident and attending physicians. Consistent with previous qualitative findings, nearly $90 \%$ of the notes in our sample were adequately complete. We did not observe significant predictors of note quality among author demographic variables decided a priori, but this finding was limited by a small sample size. Given the scope of available notes and feasibility of abstraction and assessments, future work will address these limitations through a larger audit and review of EMR notes.

Keywords: Documentation, Electronic medical records, Physicians

\section{P070}

COVID-19 pandemic and violence: rising risks and decreasing urgent care-seeking for sexual assault and domestic violence survivors
K. Sampsel, MD, K. Muldoon, PhD, K. Denize, MSc, R. Talarico, MSc, D. Fell, PhD, A. Sobiesiak, BSc, M. Heimerl, MEd

University of Ottawa, Department of Emergency Medicine, Ottawa, $\mathrm{ON}$

Introduction: There is little information on care-seeking patterns for sexual assault and domestic violence during the COVID-19 pandemic. The study objective was to examine the changes in emergency departments (ED) admissions for sexual assault/domestic violence (SADV) since the COVID-19 pandemic was declared.

Methods: ED admissions data from The Ottawa Hospital were analyzed from March 4th to May 5th (62 days) in 2020 (COVID-19 period) and compared to 2018 (pre-COVID-19) as a time matched comparison. Total and mean weekly admissions were calculated for all-cause ED admissions and for sexual/domestic violence cases. A Poisson regression (without offset term) was used to calculate the weekly case count ratio and $95 \%$ confidence intervals (CI) for the number of cases seen per week between the two time periods. Case characteristics were compared using Chi-square tests and percent differences were calculated.

Results: Compared to pre-COVID-19, total ED admissions dropped by 1111.22 cases per week (32.9\% reduction), and the SADV Program cases dropped 4.66 cases per week. The weekly case count ratio for sexual assault cases was 0.47 (95\% CI 0.79-0.27), equivalent of $53.49 \%$ reduction in cases, and 0.52 (95\% CI $0.93-0.29)$, equivalent to a $48.45 \%$ reduction in physical assault cases. The characteristics of presenting cases were similar by age (median 25 years), and sex (88.57\% female), assault type (57.14\% sexual assault, $48.57 \%$ physical assault) and location (31.43\% patient's home, $40.00 \%$ assailant's home). There was a significant increase in psychological abuse $(11.69 \%$ vs $28.57 \%)$ and assaults occurring outdoors $(5.19 \%$ vs $22.86 \%)$.

Conclusion: This study found a decrease in ED admissions for sexual assault/domestic violence during COVID-19, despite societal conditions that elevate violence risk. Trends in care-seeking and assault patterns will require ongoing monitoring to inform the provision of optimal support for individuals experiencing violence, particularly as countries begin to re-open or lock-down again.

Keywords: COVID-19 pandemic, Domestic violence, Sexual assault

\section{P071}

F-SOAP: a simple model to teach oral case presentations to medical students

A. Seto, BHSc, MD, K. Lin, MD

University of Calgary, Calgary, $\mathrm{AB}$

Innovation concept: Presenting clinical cases to preceptors is an important skill for medical students on their emergency medicine rotation. Since impressions of clinical performance may be based on how well a student presents, learners may find presenting cases to preceptors challenging and intimidating. Introducing a model to present cases may enhance students' confidence in this skill. To achieve this, the SOAP notes method of clinical documentation was adapted to create an oral case presentation model called F-SOAP. The goal was to use F-SOAP to (1) teach medical students how to present cases, (2) evaluate the F-SOAP model's ability to improve presentation confidence, and (3) determine the model's perceived usefulness.

Methods: The F-SOAP model was taught to second-year medical students through a pre-recorded podcast, which discussed the approach and included two case-based simulation exercises. Following the exercises, students used an evaluation checklist to facilitate self-reflection. 153 s-year medical students were surveyed post- 
workshop to reflect on the model's impact. Students' $(n=66)$ confidence in oral case presentation skills significantly increased ( $\mathrm{p}<0.001$ ) pre- vs post-session (mean 2.59 to $3.55 / 5.00$; mode 2.00 to $4.00 / 5.00$ ) with a large effect size (Cohen's $d=1.10$ ). Additionally, $94 \%$ of students $(n=66)$ found F-SOAP to be "useful" or "very useful"; the "usefulness" had a mean of 4.41/5.00 and was bimodal at 4.00/5.00 and 5.00/5.00. All students (64/64) planned on using FSOAP for future presentations to preceptors.

Curriculum, tool, or material: F-SOAP, an approach for oral case presentations, stands for Frame, Story, Objective Data, Assessment, and Plan.

Conclusion: F-SOAP is a simple, structured tool that appears effective in improving medical student confidence with oral case presentations. Prior to introducing F-SOAP, students expressed low to neutral confidence in presenting cases, identifying the need for a presentation approach (i.e. F-SOAP) to practice and build confidence. Although an organized model helps with student confidence, it does not help students' ability to identify pertinent positives and negatives of the case to present. Also, it is unknown if there is long-term perceived utility of F-SOAP or if students will continue using F-SOAP post-graduation. F-SOAP, however, can be adapted for other clinical uses, including consulting specialists and providing handover to peers-both common interactions in emergency medicine.

Keywords: Innovations in EM education, Oral case presentations, Teaching medical students

\section{P072}

Availability of hospital-based helipads in Ontario and impact on interfacility transfer times

E. Shachar, MD, MSc, B. Nolan, MD, MSc

University of Toronto, Toronto, ON

Introduction: Every year in Ontario, thousands of patients presenting to their local hospital require transport to another healthcare facility to receive more specialized care. This process is known as an interfacility transfer. Many of these patients will be transported by helicopter. The availability of a hospital-based helipad can significantly decrease the transport times for these patients by allowing rotor-wing aircraft to land directly at the sending or receiving hospital. The absence of a hospital-based helipad requires aircrafts to land remotely and the transporting paramedic crew to be driven into the hospital and then back to the aircraft, resulting in a significant delay to their transport. The objective of this study is to assess the availability of hospital-based helipads in Ontario, and to estimate the associated time delay if no hospital-based helipad was available.

Methods: This was a retrospective cohort study of all interfacility patient transfers by a provincial critical care transport organization over a 3-year period. Data were compiled from an administrative database and electronic patient care records. Inclusion criteria were all healthcare facilities in Ontario that manage acute medical conditions that were within the rotor-wing mission profile $(<250 \mathrm{~km}$ from a rotor-wing base). The primary outcome was the presence or absence of a hospital-based helipad. The secondary outcome used historical transport times to estimate time from landing site to patient beside.

Results: Of the 200 healthcare facilities included, 80 (40\%) did not have a hospital-based helipad. Furthermore, of the 120 facilities that did have a helipad, 48 (40\%) still required some type of land escort from the helipad to the hospital. The median overall transport time from aircraft landing site to patient bedside was $10 \mathrm{~min}$ (IQR 8.5, 17.5). Median transport times from aircraft landing site to patient bedside with and without hospital-based helipad was $10 \mathrm{~min}$ (IQR $7.25,11.75)$ and $15 \mathrm{~min}$ (IQR 11.5, 24.5), respectively.
Conclusion: The presence of a hospital-based rotor wing landing pad reduced patient transport times during interfacility helicopter transport. The clinical implications of reduced transport have not been elucidated; however, it is expected that increased availability of hospital-based landing pads will reduce overall patient transport times, which could lead to improved patient outcomes.

Keywords: Delays, Interfacility transfer, Prehospital care

\section{P073}

Efficacy of virtual simulation-based training in emergency medicine residents

K. Shani, BSc, MD, S. Wong, BSc, MD, D. Savage, BSc, MD, MSc, $\mathrm{PhD}, \mathrm{C}$. Pun, BSc, MD, R. Ohle, MBChB, MSc

Northern Ontario School of Medicine, Thunder Bay, ON

Innovation concept: Simulation-based training (SBT) is a valuable educational tool in medical training, allowing for practice and feedback in a safe and low-stakes environment. Though often done inperson using high fidelity mannequins, providing virtual SBT using teleconference platforms would be advantageous given current COVID-19 social distancing policies. However, there is a lack of studies evaluating the efficacy of a virtual simulation model. Goals of care (GOC) discussions with substitute decision makers (SDM) are important when caring for critically ill patients in the Emergency Department (ED). Review of the literature shows that SBT around GOC can be effective, though this has not been explored in a virtual format with Emergency Medicine residents. Our aim is to assess if virtual SBT can improve Family Medicine-Emergency Medicine residents' comfort and competency when discussing GOC with an SDM of an acutely ill patient in the ED.

Methods: A mixed methods study design was used to assess the efficacy of virtual SBT in the context of GOC discussion. Six Northern Ontario School of Medicine Family Medicine-Emergency Medicine residents engaged in a GOC discussion with a SDM over Zoom. Throughout the GOC discussion, updates were provided to the resident and the SDM about the patient's worsening clinical status. A debriefing session was held after the scenario. Subsequently, each resident was interviewed about their experiences with virtual SBT. The interviews were recorded, coded, and a thematic analysis was performed to identify themes relevant to using virtual SBT in Emergency Medicine training. Pre- and post-simulation surveys using a Likert scale were also used to assess efficacy of virtual SBT.

Curriculum, tool, or material: There is ongoing data collection. Expected results from our thematic analysis may include that virtual SBT is helpful with scenarios where communication is prioritized over procedural skills. The quantitative results may include that virtual SBT improves residents' overall comfort and competency in GOC discussion.

Conclusion: This is the first study to explore virtual SBT in Emergency Medicine training. Conclusions from this study may help encourage use of virtual SBT in medical curriculum.

Keywords: Innovations in EM education, Medical education, Simulation training

\section{P074}

Improving the delivery technique of respiratory metered-dose inhaler and spacer during COVID-19 pandemic and beyond

D. Shelton, MD, MSc, L. Notario, BN, V. Teo, PharmD

Sunnybrook Health Sciences Centre, Toronto, ON 
Background: Patients with asthma and COPD can present to the emergency department (ED) in respiratory distress requiring nebulized medications. Delivery of these medications using a metereddose inhaler (MDI) and spacer is not adopted in all EDs, but has become a necessity during the COVID-19 pandemic. Nebulization is an aerosolized generating medical procedure that increases the risk of infection transmission by droplet and aerosols. This necessary change in practice for some assumes that health care providers know the steps to correctly deliver inhaled medications using an MDI/spacer. 50\% of our emergency nurses did not wait 30-60 s between puffs and 50\% did not shake the MDI between puffs, thus we set out to improve this. By January 2021, 90\% of emergency nurses will correctly execute all 7 steps in use of an MDI/spacer.

Aim statement: Correct technique of MDI/spacer delivery: (1) Shake MDI prior to use. (2) Insert MDI into spacer. (3) Provide instructions to patient. (4) Place spacer mask on patient. (5) Minimize whistling with inhalation. (6) Wait 30-60 s between puffs. (7) Shake MDI between puffs Outcome measure: \% emergency nurses that correctly execute all 7 steps in use of an MDI/spacer Process measures: \% emergency nurses that correctly execute each step in use of an MDI/ spacer Balancing measure: satisfaction of emergency nurses in correct delivery technique Using PDSA cycles the following changes were introduced to teach emergency nurses how to correctly administer inhaled medication using an MDI/spacer: distribution of video demonstrating correct technique-education posters on common errors of inhaler delivery technique-reminder on automatic dispensing unit to shake between puffs-education simulation sessions. Measures and design: Post intervention strategies $100 \%$ of nurses correctly performed steps 1-4 using an MDI/spacer, $95 \%$ of nurses correctly performed step 5 (baseline $70 \%$ ), $95 \%$ of nurses correctly performed step 6 (baseline 50\%) and 65\% of nurses correctly performed step 7 (baseline 50\%).

Evaluation/results: There were improvements in all areas of deficiency, but still significant room for improvement in shaking the MDI between puffs. Failure to shake the MDI prior to each use leads to delivery of only the propellant and not the active drug. Correct use of $\mathrm{MDI} / \mathrm{spacer}$ will lead to fewer treatment failures and less use of nebulizers. This will mitigate the risk of spreading COVID-19 during its high prevalence and contribute to a sustained change in practice beyond the pandemic.

Keywords: Metered-dose inhaler and spacer, Quality improvement and patient safety, Reactive airways disease

\section{P075}

\section{Antibiotic prescription patterns for suspected urinary tract infections in the Alberta Children's Hospital emergency department}

D. Stewart, BSc, MD, S. Sudershan, BHSc, T. Fung, PhD, S. Dowling, MD

\section{University of Calgary, Calgary, $\mathrm{AB}$}

Introduction: Empiric antibiotics are commonly prescribed for presumed urinary tract infections (UTI's) in the pediatric Emergency Department (ED) setting. However, accurately diagnosing and treating pediatric UTI's poses a significant challenge in the ED, given the lack of specificity in presenting signs, symptoms and initial urine testing. We aimed to quantify the number of patients treated with an empiric antibiotic for a suspected UTI who go on to have a negative urine culture.

Methods: We performed a single centre, retrospective cohort study at the Alberta Children's Hospital ED from February to December 2019. Included children were aged 3 months to $<18$ years of age, and were discharged from the ED with a diagnosis of confirmed or suspected
UTI. Patient demographics, urine collection method, antibiotic type, urine culture results, and patient discharge instructions were also collected.

Results: A total of 395 patients, with a median age of 4.9 years were included. 199 patients $(50.4 \%)$ received antibiotics despite ultimately negative urine culture results. Cefixime was the most commonly prescribed antibiotic $(\mathrm{n}=335,84.8 \%)$. In the majority of cases $(\mathrm{n}=214,54.2 \%)$, no follow up instructions were specified in the ED discharge summary.

Conclusion: We observed that $50.4 \%$ of patients who were prescribed an empiric antibiotic for a suspected UTI had a negative urine culture. This suggests that UTI management in our ED may lead to unnecessary antibiotic exposure and highlights an important opportunity for a novel quality improvement initiative at our centre.

Keywords: Antibiotic, Pediatric, Urinary tract infection

\section{P076}

The erector spinae plane block for acute pain management in emergency department patients with rib fractures

I. Surdhar, MD, MSc, T. Jelic, MD

University of Manitoba, Winnipeg, MB

Introduction: Rib fractures represent a significant cause of morbidity and mortality in trauma patients. Opioids are commonly prescribed for patients with rib fractures however they are often ineffective which can lead to atelectasis and other complications. Recently, the erector spinae plane block (ESPB) has come to the forefront as a potential safe and effective option for analgesia in painful conditions of the thorax. This technique involves injecting local anaesthetic into the fascial plane that runs between the erector spinae muscles and the transverse processes. The anaesthetic diffuses through this plane resulting in anaesthesia of thoracic sensory nerves at multiple dermatomal levels. Because the injection point is both superficial and protected by the bony transverse process, the incidence of complications is felt to be low. This study sought to address whether this block can be used to safely and effectively provide analgesia in emergency department (ED) patients with acute rib fractures.

Methods: Patients presenting to a tertiary care ED with acute rib fractures and poorly controlled pain despite opioid therapy were recruited by convenience sampling. Patient who met inclusion and exclusion criteria were offered an ultrasound guided ESPB by one of the two study physicians using a weight based dose of $0.5 \%$ bupivicaine and saline for a total injection volume of up to $40 \mathrm{ccs}$. The primary outcome was subjective pain scores pre and post block. These were analyzed using a Wilcoxon test. Secondary outcomes were complications associated with the ESPB including block failure, shortness of breath and pneumothorax which were reported as rates of occurrence per block.

Results: 20 patients were screened for inclusion in the study, 11 patients met inclusion criteria and 2 were omitted due to inability to visualize landmarks on ultrasound. A total of 9 patients underwent the procedure. There was a statistically significant reduction in mean pre and post block pain scores (9.89 vs 3.56; $\mathrm{P}<0.0001)$. No pneumothorax or hemodynamic compromise occurred. Furthermore, there were no instances of symptomatic dyspnea.

Conclusion: This study represents the largest sample of ED patients undergoing the ESPB to date. Limitations included difficult ultrasound visualization of bony landmarks due to body habitus and subcutaneous emphysema. Overall, the results indicate that the erector spine plan block is a safe and effective technique for providing analgesia in ED patients with acute rib fractures.

Keywords: Nerve block, Rib fractures, Ultrasound 


\section{P077}

\section{Stratified stressors: quality of life significantly diminished in Canadian, female, and minority emergency clinicians during pandemic spikes and surges}

K. Suwary, BSc, H. Guertin, BSc, M. Bentley, BSc, D. Barbic, BSc, MD, MSc, K. Rocklein Kemplin, BSc, MSc, PhD, DNP

Schulich School of Medicine, Windsor, ON

Introduction: Pre-pandemic, Emergency Medicine (EM) clinicians were exponentially higher in expressions of post-traumatic stress (PTS) than other specialties. Now, Quality of Life (QoL) is a rediscovered construct of concern in the midst of the COVID-19 pandemic as frontline clinicians buckle under the weight of unrelieved psychosocial burdens, diminished resilience, and unrelenting professional demands. Emergency departments morphed into battlegrounds upon which much of the war against COVID-19 is fought: first-person video and media reports highlight impacts on EM clinicians' QoL worldwide. Thus, we hypothesized EM QoL differs between clinicians' country, role, and other demographic strata.

Methods: We employed a cross-sectional design, administering the WHOQOL-BREF instrument via electronic distribution to EM clinicians in Canada and abroad. Responses were anonymous. Data were collected from April to June 2020. Our primary outcome was quantifying QoL among EM clinicians during initial pandemic surges, whereas our secondary outcome was to psychometrically analyze this instrument within these participants contextually during COVID-19.

Results: Canadian clinicians reported suboptimal QoL in comparison to their American counterparts. Interdisciplinary EM participants $(\mathrm{N}=302)$ had significantly different $(\mathrm{p}<0.05)$ QoL among physicians, nurses, females, and younger clinicians $(<39$ years old $)$ and those identifying as minorities: deteriorating mental and physical health, higher personal safety concerns, and financial strains. Internal consistency was robust with a Cronbach's alpha of 0.883 , however, factor analyses loaded onto one main component with an eigenvalue of 9.102 and $59.9 \%$ variance. Statistical power of 0.80 was reached at $\mathrm{N}=256$

Conclusion: Diminishing QoL occurred during initial pandemic surges and will likely plummet again in subsequent, more acute waves despite anticipated mass vaccinations. Psychometrically, the WHOQOL-BREF is likely imprecise in capturing QoL among moreaffluent participants whose basic needs are met regardless of professional stressors or public health crises. Quasi-experimental research should ensue after implementation of targeted interventions designed to ameliorate EM clinicians' deteriorating QoL, especially given extant baseline suicidality and expressions of PTS in EM, which supersedes other specialties.

Keywords: COVID-19 pandemic, Emergency medicine, Quality of life

\section{P078}

Developing a dashboard for faculty development in competencybased training programs: a design-based research project

B. Thoma, MD, MSc, MA, Y. Yilmaz, PhD, R. Carey, BSc, MD, V. Bandi, MSc, S. Wang, BSc, D. Mondal, PhD, R. Woods, MD, MMEd, T. Chan, MD, MHPE, B. Thoma, MD, MSc, MA

University of Saskatchewan, Saskatoon, SK

Introduction: Canadian specialist residency training programs have implemented competence by design, a form of competency based medical education (CBME) that requires frequent assessments of entrustable professional activities. However, faculty struggle to provide helpful feedback and assign appropriate entrustment scores. Audit and feedback programs have demonstrated some efficacy in engaging clinicians to change behavior, but faculty development initiatives related to CBME rarely incorporate assessment metrics. Methods: Within the University of Saskatchewan emergency medicine program, we utilized a design-based research process to identify faculty development needs related to CBME assessments and design a dashboard containing elements (data, analytics, and visualizations) meeting these needs. Data was collected within the emergency medicine residency program at the University of Saskatchewan through interviews with program leaders, faculty who had participated in an audit and feedback session, and faculty development experts. Two reviewers analyzed interview transcripts using a grounded theory approach to identify faculty development needs that were vetted by a third reviewer. The needs were described using representative quotes and addressed through the development of dashboard elements that were developed and refined throughout the study period.

Results: Participants included two program leaders (6 interviews), three faculty development experts (5 interviews), and four faculty members (4 interviews) held between July 1, 2019 and December 11, 2020. Faculty needs were classified under three themes: Analyses of assessments organized by assessor (addressed through the development of a dashboard page presenting the quantitative and narrative assessment data of individual faculty), contextualization of assessorspecific analyses (addressed by contextualizing this data by peer, rotation, time, and resident), and accessible reporting of the analyses (addressed through the export of reports for faculty).

Conclusion: Our design-based research process identified faculty development needs and created dashboard elements to meet them. The use of the resulting dashboard for audit and feedback sessions was endorsed by the participating faculty members. This work will inform the creation and evolution of CBME assessment dashboards capable of the data-driven identification of struggling faculty and support of their development.

Keywords: Competency based medical education, Faculty development, Learning analytics

\section{P079}

Gender equity in academic medicine: a review of expert generated top emergency medicine studies

A. Tolmie, BSc, A. Tan, MD, BMSc, A. Chin, MD, MSc, T. Chan, MD, MHPE

University of Saskatchewan, Saskatoon, SK

Introduction: Despite a relatively equitable gender distribution of trainees, inequity in authorship of papers remains a long standing issue in academic medicine. The roles of first and senior/corresponding author of papers are usually seen to be of primary importance for granting agencies and promotion procedures at many institutions. In 2019, a Canadian group empirically determined a list of top papers for emergency medicine junior learners. Our study's objective was to assess the gender distribution of first and senior/corresponding authors of these studies.

Methods: Contributors to all 29 original research papers identified the 2019 study were listed under author, first author, and senior/corresponding author. Researchers were included if listed as an author, or member of the writing committee. Senior authorship was determined objectively through a standardized criteria. Genderize.io, a program which utilizes international data to assign gender and probability to names, was applied to the list of authors.

Results: In total, 393 names were included as authors to the identified papers, which were published between 1992 and 2016. Of those, 
$23.9 \%$ were determined likely to be female gender, $75.1 \%$ male, and $1.0 \%$ unknown. 29 first authors and 29 senior authors were also determined. Of those, only $6.9 \%$ of first authors and $17.2 \%$ of senior authors were assigned a female gender through genderize. When grouping studies based on publication year, female author prevalence ranged from 12.5 to $41.7 \%$, with no appreciable trend throughout the year categories.

Conclusion: In conclusion, there is a large gender discrepancy between authors of these top papers, which is even more pronounced in first and senior authorship. This is likely multifactorial given the gender discrepancies in salary and research funding, and lackluster accommodation for individuals requiring a flexible work schedule. Therefore, systemic changes are required to address these gender inequities in academic medicine, and empower all of our colleagues to achieve.

Keywords: Academic medicine, Gender equity

\section{P080}

An evaluation of emergency department pain management practices for fragility fractures of the pelvis and their impact on patient outcomes

U. Tran, MD, MSc, K. Yadav, MD, MSc, M. Ali, BSc,

M. Austin, MD, M. Nemnom, MSc, D. Eagles, MD, MSc

University of Ottawa, Department of Emergency Medicine, Ottawa, $\mathrm{ON}$

Introduction: Fragility pelvic fractures are a common emergency department (ED) presentation in older patients. Adequate pain control is crucial for achieving good outcomes. However, there is little research to guide this area. The primary objective of this study was to describe current pain management practices for ED patients with fragility pelvic fractures.

Methods: We performed a health records review using consecutive sampling of adults $\geq 65$ years old who presented to two tertiary care EDs with fragility pelvic fractures that were managed nonoperatively. The study period was January 2014 to September 2018. A standardized case record form was used to extract prehospital and hospital data. The primary outcome measures were type and timing of analgesic medications. Secondary outcome measures included consultation to ancillary services, ED length of stay, admission rate and rate of return to ED within 1 month of discharge. Data was reported using descriptive statistics.

Results: We included 411 patients. The majority were female (339, $82.5 \%)$; the mean age was 83.9 (SD 8.1 ) years; and most $(244,59.4 \%)$ were from home. Nearly one third $(130,31.6 \%)$ did not receive any analgesia in the ED for their fracture. Analgesia was initiated in 69 $(16.8 \%)$ patients prior to physician assessment through paramedic and nursing medical directives. There were 244 (59.4\%) patients who received physician-initiated opioids (hydromorphone and/or morphine) for their pain. In total, $95(23.1 \%)$ patients received one or more ancillary services in the ED, which included physiotherapy $(10.5 \%)$, social work $(7.3 \%)$, geriatric nurse assessment $(14.1 \%)$, and homecare $(3.9 \%)$. Mean ED length of stay was 11.6 (SD 7.1) hours and $210(51.1 \%)$ patients were admitted to hospital. Within 1 month of discharge from the ED, 45 (22.4\%) patients returned, of whom 19 $(42 \%)$ returned because of injury related pain.

Conclusion: Our study was the first to describe current approaches employed in the prehospital and ED settings to manage nonoperative fragility pelvic fractures in older patients. A significant proportion of patients received no analgesia. Overall, we highlighted a need for further improvements to optimize analgesia and for future research to identify the barriers to appropriate acute pain management in older patients with fragility pelvic fracture.
Keywords: Fracture, Older, Pain

\section{P081}

An exploration of Canadian emergency physician personal experiences during the COVID-19 pandemic

A. Tran, BSc, C. Wallner, MD, MSc, K. de Wit, MD, MSc, S. Gray, BSc, MD, MPH, P. Archambault, MD, MSc, K. Ritchie, PhD, T. Chan, MD, MHPE

McMaster University, Hamilton, ON

Introduction: Emergency physicians have high rates of burnout, depression, and suicidality and are at the forefront of the COVID-19 pandemic. While initial results of the EmergWell cohort study suggest lower rates of burnout among Canadian Physicians in the initial 10 weeks of the pandemic response, we aimed to further explore the ongoing personal experiences of emergency physicians during the pandemic.

Methods: We conducted a national, qualitative study between May and September, 2020. Emergency physicians working in Canada were invited to participate in a cohort survey-based study which was advertised through national and provincial professional societies, as well as through Twitter and Facebook. Participants were further invited to a qualitative interview to explore their ongoing experiences. Interviews took place via Zoom and were transcribed verbatim. The interviewer was a qualitative researcher without medical qualifications. Three researchers (the interviewer and two emergency physicians) performed thematic analysis of the transcripts.

Results: A total of 468 physicians throughout Canada participated in the survey phase. Qualitative data from the initial surveys were supplemented by individual interviews with 16 participants (11 female, 5 male). Feelings of fear and worry were associated with working in an unusually high-risk environment and the potential for exposure of COVID-19 to their families. As initial patient volumes decreased, these feelings were balanced by a renewed sense of purpose in their work and a new feeling of support both socially and within the clinical environment. However, intensive pandemic preparation such as the running of simulations, required long work hours and challenged work-life balance. Subsequently, the return of patient volumes and a stressed medical system with ongoing expanded non-clinical duties contributed to increasing anxiety and frustration related to personal protective equipment complacency and a return to pre-pandemic behaviours. Participants reported prolonged isolation and inability to access typical coping strategies, such as exercise. This, along with a sense of an impending second wave, all contributed to the erosion of that initial enthusiasm.

Conclusion: Physicians had a renewed sense of purpose at the outset of the pandemic. However, we found increasing anxiety, isolation, and fear among emergency physicians as the first pandemic wave progressed.

Keywords: Anxiety, Burnout, Pandemic

\section{P082}

Creation of a novel evaluation tool to manage conflict of interests in clinical practice guideline panels

S. Upadhye, MD, MSc, C. Carpenter, MD, MSc, J. Yan, MD, MSc, E. Lang, MD, S. McLeod, PhD, J. Morris, MD, MSc, G. Ghate, MD, MSc, C. Davies-Schinkel, MSc, RN, S. Strobel, MD,

D. Kumbhare, $\mathrm{MD}, \mathrm{PhD}$

McMaster University, Hamilton, ON 
Introduction: Conflicts of interest (COI), of all types, threaten the trustworthiness of clinical practice guidelines (CPGs), but current $\mathrm{CoI}$ management strategies are inconsistent. The objective of this study was to develop a new decision tool to manage COI among CPG panelists.

Methods: Common COI elements were categorized into 3 conflict categories: financial (14 domains), academic (5) and personal (4). A reporting form including all potential conflicts was created for panelist completion. A hypothetical panel of 13 panelists was constructed with various domain mixes of reported COI, including details of conflicts. A group of 7 research experts with experience in CPG panels were recruited and trained to complete the ratings of the hypothetical panelists. Raters answered 3 key COI management questions for each panelist: (1) what is the Global Risk of Bias (RoB) associated with each panelist, (2) should the panelist be included or excluded from discussing CPG recommendations, and (3) should the panelist be included or excluded from voting on CPG recommendations? Results were pooled and analyzed for consensus ratings and decisions. Inter-rater reliability for agreement was calculated using generalizability theory, including a D-study for optimal number of raters.

Results: The overall inter-rater reliability for the COI-RoB instrument was 0.92 (95\% CI 0.86-0.95), with a D-study showing an optimal number of 7 raters. "Global Risk of Bias" scores were highly congruent for non- or highly-conflicted panelists, but more variation was noted for different panelists with different conflict categories. Raters were more permissive to include conflicted panelists in "discussing" CPG recommendations (excepting the highly financially conflicted panelist). Raters were much more restrictive, however, in allowing conflicted panelists to participate in "voting" for CPG recommendations.

Conclusion: This new COI-RoB tool demonstrates high inter-rater reliability, and can be used by CPG panel Chairs to assess and adjudicate individual panelist reported conflicts in a thorough and transparent manner. Individual panelist conflict reports and panel adjudications can be included in CPG publication supplementary materials, so that readers can view such transparent reporting with increased understanding and confidence in panel methods.

Keywords: Clinical practice guidelines, Conflict of interest

\section{P083}

You might have COVID-19: improving patient education for behaviour change using information design

S. Vaillancourt, BA, MD CM, MPH, K. Sellen, BSc, MSc, PhD, MRes, S. Gupta, MD, C. Rice, BComm, MDes, Y. Ghader, BID, G. Ginocchio, HBSc, A. Ceccacci, BHSc

\section{St. Michael's Hospital, Toronto, ON}

Innovation concept: In March 2020, a large number of patients with symptoms concerning for COVID-19 started presenting to emergency departments (EDs) and rapid assessment centres. Patients were asked for drastic behaviour change with limited interventions. The patient information available relied on text, had limited language availability, and used complex nomenclature and instructions. This made comprehension difficult, even for healthcare professionals. We sought to develop easy to understand and adaptable information sheets (printables) to guide patients with symptoms of COVID-19 in self-care and general guidance on self-isolation.

Methods: Rapid iterative design method was used, using principles of inclusive design, to develop the printables. The SURF framework on patient outcomes after ED care was incorporated, as was prior research on designing effective communication materials for patients/family caregivers. The rapid design cycle engaged across disciplines including emergency physicians, public health professionals, and health communication designers. Aware of the need for rapid scaling and spread, we took an open innovation approach to dissemination.

Curriculum, tool, or material: Licensed under creative commons, the printables are non-branded, non-jurisdictional, are available for download, and are easily editable. This enables rapid adoption and easy updating for changing guidelines or local circumstances. Designed for easy printing and handoff with a simple 1 page, black and white design, the printables are dominated by infographics that are simple, consistent, and friendly, with no discernable gender or race. All text is low literacy friendly and supportive (i.e. positive enabling, avoiding negative and authoritarian language or tone). The information design is highly structured with consistent blocks to aid comprehension and memorability. All materials incorporate a call to action, the result of rapid research on COVID behavior change messaging.

Conclusion: There has been an international response to the printables, with over 3000 website visits in 1 week during March, 2020, and an international award. The printables have seen continuous use in our emergency department and across Canada. Now professionally translated into 45 languages, there are 8 printables, filling a gap in health communication. A partnership with community organization Access Alliance has led to wide scale distribution among health partners beyond the emergency department.

Keywords: Behavior change, Health communication design, Innovations in $\mathrm{EM}$ education

\section{P084}

\section{Extending the emergency department during a pandemic}

S. Vaillancourt, BA, MD CM, MPH, R. Girdhari, BSc, MBA, MD CM, K. Chalklin, BA, MHE, J. Chen, MN, N. Dewhurst, PharmD, C. Kokoski, BN, BSc, MSc, K. Weyman, MD, MEd, C. Snider, MD, $\mathrm{MPH}$

St. Michael's Hospital, Unity Health Toronto, Toronto, ON

Background: The Covid-19 pandemic threatened the ability of emergency departments (EDs) to maintain the provision of quality care. In March 2020, our department faced the imminent threat of a surge in patients affected by Covid-19 and an increased need for space for patient distancing. We aimed to be ready within 2 weeks of March 252020 to divert a majority of low acuity patients from the main ED site.

Aim statement: Our main outcome measure was the number of patients diverted from the main ED space. Process measures included: date of space readiness and number of staff oriented and available. Balancing measures: adverse events away from appropriate clinical settings.

Measures and design: A close collaboration was established between the departments of family and emergency medicine with joint leadership and staffing commitment. A large outpatient clinic space left unused by the sudden transition toward virtual care was repurposed as the ED Low Acuity (ED LAc). Triage, registration and patient transport was planned through iterative process maps. The new space was adapted to its new use, including ED equipment, stocking and physical distancing accommodation. Thirty-eight family physicians were oriented to ED LAc, with 12 rostered for phase 1. Nursing and clerical staffing was ensured by redeployed staff with and without ED experience. A mixed clinical staffing model, new space and workflow were tested through two In-situ simulations, revealing gaps in code blue procedures and patient ED repatriation. The space was ready within 2 weeks. 
Evaluation/results: Since the ED patient volumes had decreased by over $50 \%$, the ED LAc opening was postponed by 2 weeks to April 162020 and limited to $8 \mathrm{~h}$ a day with a planned staged ramp up. Emergency and family physicians were paired for clinical orientation. Low numbers of patients (4-26) received care in ED LAc, reflecting the historically low ED attendance seen during this phase of the COVID-19 pandemic in Ontario. Staffing was reduced to a single physician on May 4, 2020 and ED LAc was suspended on May 29 with protocol plans archived for future re-deployment as needed. No adverse event was recorded. The ED LAc successfully increased ED capacity in a very short time frame, but ultimately turned out to not be needed. Key enabling factors were strong institutional support and leadership, collaboration across departments, iterative process mapping and testing through simulation, and the development of a staged deployment that could match resource utilization to demand.

Keywords: COVID-19, Disaster response, Quality improvement and patient safety

\section{P085}

Co-designing discharge information after emergency care with healthcare providers and patients

S. Vaillancourt, BA, MD CM, MPH, S. Gupta, MD, Y. Ghader, BID, C. Rice, BComm, MDes, A. Ceccacci, BHSc, V. Weng, BSc, G. Ginocchio, HBSc, M. Beaubien, BDes, K. Sellen, BSc, MSc, PhD, MRes

\section{St. Michael's Hospital, Toronto, ON}

Background: Patients are most often discharged home after emergency department (ED) care with only verbal communication. Prior research defined this as an important problem to patients, their care partners, and primary care providers. Despite many attempts at introducing written materials, the vast majority of patients at the St. Michael's Hospital ED receive only verbal information. We sought to design a patient-centered discharge information process that would maximize adoption and sustainability.

Aim statement: The objective was focused on improving four patient outcomes of importance to ED patients defined in the PROM-ED study: Symptom relief, Understanding, Reassurance, and Having a plan for follow up. We developed a core team of designers and clinicians. We performed a review of existing ED discharge tools. Three assistants completed field observations in the ED and participative journey mapping was subsequently completed. Co-design sessions were facilitated virtually. A prototype solution was then tested through cognitive debriefing and cycles of user feedback and refinement.

Measures and design: Our literature search identified 41 existing discharge tools for ED care which were reviewed. Three assistants completed 63 observation sessions in the ED. The care journey was mapped to identify opportunities for the transfer of information including patient registration, longitudinal ED experience, and final discharge. A prototype was developed and presented over 4 co-design sessions that involved 19 participants divided between patients (6), ED nurses (5), other ED staff (2), family physicians (2), and ED physicians (4). A solution in the form of a patient-oriented chart (POC) was identified as most promising and developed into a prototype. The POC was tested in actual use in the ED and iteratively revised 6 times based on feedback from users and the co-design participants. A late-stage prototype was tested with 7 scenarios through cognitive debriefing with patients in the ED and led to a finalized solution ready for implementation.

Evaluation/results: We co-designed a solution to improve discharge information with patients and providers that is focused on improving well-defined patient outcomes and maximizing adoption through consideration of local technological and workflow constraints. The patient-oriented chart provides a template for efficient customized patient-centered discharge information in paper or digital format at the conclusion of ED care.

Keywords: Design, Patient-reported outcome measures, Quality improvement and patient safety

\section{P086}

A descriptive analysis of pediatric CTAS 1 patients presenting to a community ED who require transfer to a pediatric centre

R. Valani, MBA, MD, MEd, S. Upadhye, MD, MSc, M. Khalid, BSc, H. Snyder, BSc, T. Sharma, BSc, MSc, S. Brown, BSc, MD

University of Toronto, Toronto, ON

Introduction: Pediatric patients usually compromise $20-30 \%$ of community ED visits. Their management can vary depending on local resources and the comfort level of the emergency physician. Specifically, high acuity CTAS I pediatric patients require additional resources that necessitate transfer to a regional pediatric centre. The purpose of this descriptive study was identify predictors of the CTAS I patients needing transfer.

Methods: This was a retrospective chart review of all pediatric CTAS I patients presenting to the Niagara Health Region from Jan 2017 to December 2018. Descriptive statistics were used to understand the population. Binary regression using age, gender, Pediatric Early Warning Score (PEWS), season, and time of day were used to identify predictors for transfer to a tertiary care centre.

Results: There were 1,137 patients who presented to the region during the time period, with an average age of 5.08 years (SD 5.02) of which there were 559 male $(49.2 \%)$ patients. The median length of stay in the ED was $174 \mathrm{~min}$ (IQR 99-268 min). Of the entire cohort, sixty patients $(5.3 \%)$ were transferred to a tertiary care centre. Predictors for transfer included age (OR 1.1), female gender (OR 0.9), higher PEWS score (OR 1.4), summer months (OR 1.8) and daytime (OR 1.2). Only age and PEWS score were statistically significant $(\mathrm{P}<0.05)$.

Conclusion: This is the first study looking at predictors for transfer to a regional pediatric centre from a community setting of pediatric CTAS I patients. Not surprisingly, PEWS and age were strong predictors for the need to transfer. Further studies are required to identify additional predictors that can help facilitate quicker transfer from the time of triage of these patients.

Keywords: CTAS, Pediatric, Transfer

\section{P087}

Causes and predictors of early postpartum complications that result in visits to the emergency department

R. Valani, BSc, MBA, MD, MEd

University of Toronto, Toronto, ON

Introduction: This study aimed to review the reasons why postpartum women present to the emergency department (ED) over the short term ( $\leq 10$ days post-delivery) and to identify the risk factors associated with early visits to the ED.

Methods: This was a retrospective study that included deliveries at the William Osler Health System in 2018 and presented to the ED within 10 days after delivery. Descriptive statistics were used to examine postpartum visits. Multiple binary regression was used to identify predictors of early visits to the ED. 
Results: Overall, 429 unique postpartum ED visits occurred over 1 year (2018), of which 381 were included in this analysis. The average age of the patients was 31.22 years (SD 4.83), and the median gravidity was 2 (IQR 1-3). Most patients delivered via spontaneous vaginal delivery $(52.36 \%)$. The median time of presentation to the ED was 5.0 days, with the most common reasons as follows: abdominal pain $(17.02 \%)$, wound-related issues $(13.09 \%)$, and fever or vaginal/ rectal pain $(9.95 \%)$. Weekend $(\mathrm{P}<0.1)$ and $\mathrm{C}$-section (cesarean) deliveries $(\mathrm{P}<0.001)$ were significant predictors of $\mathrm{ED}$ visits. Only one-quarter of the cases required an obstetric consultation, and $85.86 \%$ of all patients were discharged.

Conclusion: This study was the first in a busy community setting that examined ED visits over a short postpartum period. Patient education on pain management and wound care, including the availability of an early postpartum contact, can potentially decrease the rate of ED visits.

Keywords: Emergency, Postpartum complications, Visit

\section{P088}

Pandemic perspectives: reductions in respiratory and gastrointestinal communicable diseases in Calgary adult emergency departments

M. Varma, BSc, H. Walia, MD, S. VandenBerg, MD, MSc, E. Lang, MD CM

University of Calgary, Calgary, $\mathrm{AB}$

Introduction: While emergency departments across Canada prepared for the influx of COVID-19 patients, the actual experience of emergency departments (EDs) in Calgary was a decrease in patient volumes. This study explores which specific patient presentations trends were the most striking and offers suggestions for why they may have occurred.

Methods: This study specifically evaluates the trend in emergency department visits for respiratory and gastrointestinal communicable diseases using a purpose built software (AHS Tableau) to trend specific illness presentations based on administrative data. Data was collected on ED presenting complaints between May 2019 and November 2020. Pre-COVID and post-COVID timeframes were compared.

Results: We observed that, pre COVID-19, the average daily visits for the respiratory and gastrointestinal related complaints was recorded at 539 unique visits from November 2019 to January 2020 which dropped to 110 average daily visits from March to November 2020. This trend was noticed in complaints like cough/congestion which fell, on average, from 268 to 28 (90\% decrease), nausea/vomiting which decreased from 114 to 36 (68\% decrease) and fever presentations which decreased from 132 to 28 ( $79 \%$ decrease).

Conclusion: The initial wave of COVID-19 resulted in fewer emergency department visits for respiratory and gastrointestinal communicable diseases. This is interesting given the cross over between the respiratory symptoms of COVID and the chief complaints documented for the ED visits.

Keywords: Gastrointestinal illness, Pandemic, Respiratory illness

\section{P089}

Development and evaluation of an online simulation curriculum for interdisciplinary medical teams at electronic dance music festivals

A. Vorobeichik, BSc, A. Seto, BHSc, MD, L. Haynes, BHSc, A. Hussein,, W. Kennedy, BSc, MD
University of Calgary, Calgary, AB

Innovation concept: Electronic Dance Music (EDM) festivals present unique challenges to patient care, including resource limitations, loud music, crowded environments, and working with ad hoc teams to manage uncommon clinical cases. The objectives were to develop an online, interdisciplinary simulation curriculum and evaluate its impact on healthcare providers' self-efficacy for, and knowledge in managing emergency medical cases at EDM festivals.

Methods: Healthcare providers/trainees from various backgrounds (e.g., medicine, nursing, paramedicine) participated in three consecutive online simulations (cardiac arrest, serotonin toxicity, and toxininduced seizure) in teams of 4-5. Two simulation facilitators managed case progression through a screen-share of Google Forms and teams communicated through Zoom. Participants completed quantitative self-efficacy and knowledge assessments 1 week pre- and postsession, as well as a qualitative survey immediately post-session, reflecting on teamwork, take-home learning points, and curriculum satisfaction.

Curriculum, tool, or material: Mean self-efficacy ratings $(n=18)$ increased pre- vs post-session (one-tailed repeated-measures $t$ test) for cardiac arrest (3.80 to 4.31/5.00; $\mathrm{p}<0.01$; Hedges' $\mathrm{g}=0.64$ ), serotonin toxicity $(2.60$ to $4.13 / 5.00 ; \mathrm{p}<0.001 ; \mathrm{g}=1.63)$, and seizure ( 2.70 to $3.83 / 5.00 ; \mathrm{p}<0.001 ; \mathrm{g}=1.03$ ). Mean knowledge quiz scores $(\mathrm{n}=18)$ increased pre- vs post-session (one-tailed repeatedmeasures $t$ test $)$ for all subsections $(\mathrm{p}<0.001)$ : cardiac arrest $(67-$ $80 \%, \mathrm{~g}=0.59)$, serotonin toxicity $(65-83 \%, \mathrm{~g}=0.86)$, and seizure $(54-76 \%, \mathrm{~g}=0.82)$. Thematic analyses identified collaborative decision-making and communication as reported teamwork strengths. Commonly reported take-home learning points were related to pharmacology/toxicology, clinical approaches, and differential diagnoses. Average program satisfaction rating $(n=22)$ was 4.36/5.00.

Conclusion: The online simulation curriculum increased participants' self-efficacy and knowledge on tailored learning objectives 1 week post-session. Online simulation can be an educational and satisfying training experience for festival medical teams and offers several benefits: (1) serves as an icebreaker for teams prior to arriving at events, (2) eliminates physical distractions (e.g., loud music, crowding) to focus on clinical knowledge and approaches, and (3) offers convenience and cost-savings. This curriculum may provide a framework for future applications of online simulations in mass gathering medicine.

Keywords: Online simulation, Innovations in EM education, Interdisciplinary

\section{P090}

Optimal management strategies for primary headache in the emergency department

S. Wells, MD, I. Stiell, MD, MSc, E. Vishnyakova, BSc, R. Lun, MD, M. Nemnom, MSc, J. Perry, MD, MSc

Department of Emergency Medicine, University of Ottawa, Ottawa, ON

Introduction: Primary headaches are a common emergency department (ED) presentation. ED physicians should aim to treat primary headaches in an effective and rapid fashion to optimize ED flow and improve patient experience. We sought to evaluate the factors associated with better outcomes for ED patients treated for primary headache.

Methods: This was a health records review of consecutive patients over a 3-month period presenting to two tertiary EDs and discharged with a diagnosis of primary headache. Our main outcome was the need for second round medications, defined as medications received 
more than an hour after the initial physician-ordered medications were administered. Our secondary outcome was a long length of ED stay, defined as greater than $3.5 \mathrm{~h}$ from physician assessment to discharge. We performed multivariate logistic regression analysis to determine treatment factors associated with need for second round medications and prolonged length of stay.

Results: We included 553 patients, mean age was 42.2 years and $72.9 \%$ were female. The most common diagnoses were headache not otherwise specified $(48.8 \%)$ and migraine $(43 \%)$. Ketorolac IV $(62.2 \%)$ and metoclopramide IV $(70.2 \%)$ were the most frequently administered medications. $18 \%$ of patients met the primary outcome of receiving second round medications. After multivariate regression analysis, dopamine antagonists (metoclopramide, haloperidol) [OR 0.3 (95\% CI $0.1-0.5)$ ] and NSAIDs [OR 0.5 (95\% CI $0.3-0.8$ ) ] ordered with initial medications were associated with reduced need for second round medications. IV fluid boluses $\geq 500 \mathrm{ml}$ [OR 2.8 (95\% CI 1.5-5.2)] and anti-emetics (dimenhydrinate, ondansetron) [OR 2.2 (95\% CI 1.2-4.2)] were associated with higher rates of receiving second round medications. Opioid use approached statistical significance for receiving second round medication [OR 1.99 (95\% CI 0.98-4.05]) First round NSAIDs [OR 0.6 (95\% CI 0.4-0.9)] were associated with shorter lengths of stay. First round anti-emetics [OR 2.5 (95\% CI 1.3-4.8)] and ED investigations [OR 5.1 (95\% CI 3.1-8.2)] were associated with prolonged length of stay.

Conclusion: We determined that use of dopamine antagonists and NSAIDs reduced the need for second round medications in ED patients treated for primary headache. Conversely, anti-emetics and intravenous fluids were associated with a significantly increased rate of second round medication use. Careful choice of initial therapy may optimize management for these patients.

Keywords: Emergency department, Headache, Migraine

\section{P091}

\section{Patient perspectives of care received for sickle cell disease in Ontario hospitals}

S. Williams, MD, MSc, M. Kirby, MD, M. Verhovsek, MD, L. Tunji-Ajayi, M. Darkwa, S. Jemitola, Dr

Hospital for Sick Children, Toronto, ON

Introduction: $55 \%$ of individuals with sickle cell disease in Canada, approximately 2750 people, reside in Ontario. Hospital visits, including emergency room visits, are a common component of health care for sickle cell disease. This study sought to gather information on patient perspectives of care received, to identify areas which could be targeted for improvement.

Methods: An online survey was created via Survey Monkey and disseminated. 2500 patients and caregivers across Ontario were informed of the study via the Sickle Cell Association of Ontario Newsletter mailing, postings of the study on the Sickle Cell Association of Ontario WhatsApp forum, posting at community health centres with sickle cell programs and notification via other sickle cell associations in Ontario. From July 2019 to February 2020, participants were able to submit health care experiences via the online survey. Results were summarized descriptively.

Results: A total of 66 responses were collected. Of the 66 responses, 20 were complete, with all 49 survey questions answered. Participants reported on experiences at 14 hospitals in Ontario. Respondents were 18-64 years of age. 80\% (16/20) participants reported on experiences in the emergency room. 65\% (13/ 20) of the reports were regarding care for pain management. With respect to wait times over $1 \mathrm{~h}, 31 \%$ reported waiting greater than $1 \mathrm{~h}$ for nurse contact, $56 \%$ for physician contact, $50 \%$ for medication administration. $60 \%$ reported the first person encountered at triage was respectful, empathetic, and caring. 35\% of respondents felt some form of stigmatization and fear of dying during their hospital experience. $40 \%$ of respondents felt loneliness or helplessness during the hospital visit. $30 \%$ of respondents reported that the health care provider was extremely or very responsive to their concerns, $50 \%$ that the health care provider was somewhat responsive to their concerns, $20 \%$ that care provider was not responsive to their concerns. $30 \%$ of respondents reported that the health care providers were very knowledgeable, $50 \%$ of respondents reported the health care providers were somewhat knowledgeable, and $10 \%$ reported the health care provider as not all knowledgeable.

Conclusion: A survey on care received in Ontario hospitals identified potential areas for improvement, as defined by patients, in optimizing sickle cell disease care; including wait times to receipt of care, and perceived health care provider responsiveness to patient concerns.

Keywords: Patient care, Patient perceptions, Sickle cell disease

\section{P092}

Intubation performance and team safety using novel COVID-19 protocols during high-fidelity simulation are equal between an urban tertiary-care and a community emergency department: a prospective cohort study

S. Wong, BSc, J. Macleod, MD, M. Li, BN, BSc, F. Tenorio, BN, BSc, L. Farina, RRT, C. Gagnon, RRT, K. Dullemond, MD, C. Renschler, MD, J. Yoo, MD

University of British Columbia, Vancouver, BC

Introduction: The COVID-19 pandemic has highlighted the need for balancing timely care to patients requiring endotracheal intubation (ETI) with adequate safety measures for health-care providers (HCP). Equipment preparation and donning PPE take time and have direct safety implications for HCPs. Shorter times to successful ETI are associated with better patient outcomes. We performed a prospective cohort study of HCP performance and safety during ETI in highfidelity simulations between a tertiary-care and community ED using COVID-19 protocols. We hypothesized that there would be no significant differences in speed, performance, or contamination during ETIs between the sites.

Methods: High-fidelity simulations were performed in the two EDs using the same institutional COVID-19 ETI protocols. Simulation teams included an emergency physician, registered nurse, and respiratory therapist. Simulations were video recorded and times needed to complete key tasks were calculated retrospectively using timestamps. Fluorescent fluid was emitted from the mannequin's airway during ETI and UV lights detected areas of contamination on PPE and participants, pre- and post-doffing respectively. The primary outcome was first-attempt success (FAS). Secondary team outcomes were the time needed to prepare equipment and medications, and total time to successful ETI. Secondary individual outcomes were the time needed to don PPE and the frequency of contamination post-doffing.

Results: The tertiary-care ED had 5 simulations (15 participants) and the community ED similarly had 6 simulations (18 participants). There was no significant difference in the primary outcome of FAS rate $(80.0 \%$ vs $83.3 \%, \mathrm{P}=0.90)$. There were no significant differences in secondary team outcomes: mean times needed to prepare equipment and medications ( 2.28 vs $2.18 \mathrm{~min}, \mathrm{P}=0.84)$ and mean time to successful ETI (13.28 vs $13.40 \mathrm{~min}, \mathrm{P}=0.95$ ). There were no significant differences in individual secondary outcomes: mean time needed to don PPE (4.16 vs $3.94 \mathrm{~min}, \mathrm{P}=0.32$ ), and the frequency of contamination post-doffing ( 0 vs $1, \mathrm{P}=0.33$ ). 
Conclusion: COVID-19 ETI protocols and enhanced PPE are critical for protecting HCPs from disease transmission. Simulation teams at tertiary-care and community EDs demonstrated equal performance and safety using novel protocols. ETI was performed with a high level of success and evidence of post-doffing contamination was rare. A standardized COVID-19 protocol for ETI in the ED can be effective in different clinical settings.

Keywords: COVID-19, Intubation, Simulation

\section{P093}

The lived experience of emergency department patients after a visit for hyperglycemia: implications for communication and factors affecting adherence post-discharge

J. Yan, MD, MSc, D. Azzam, BSc, M. Columbus, PhD, K. Van Aarsen, MSc, S. Liu, MD, MSc, T. Spaic, MD, MSc, L. Shepherd, MD, MHPE

Western University, London Health Sciences Centre, London, ON

Introduction: While studies have reported factors affecting adherence to diabetic care plans from a chronic disease perspective, no studies have addressed issues with post-discharge adherence facing patients with diabetes after an emergency department (ED) presentation for hyperglycemia. This study's objectives were to describe the patient experience surrounding discharge after an ED visit for hyperglycemia and to identify factors that influence post-discharge adherence.

Methods: We conducted a qualitative description (QD) study of adult patients who had visited the ED for hyperglycemia. Consistent with QD, purposive sampling was utilized, seeking diversity across age, gender and diabetes type. Participants took part in semi-structured interviews and thematic analysis was used to identify and describe core themes. Frequent team meetings were held to review the analysis and to develop the final list of themes used to re-code the dataset. Analytic insights were tracked using reflective memos and an audit trail documented all steps and decisions.

Results: 22 patients with type 1 and 2 diabetes were interviewed. Participants identified several factors that impacted their ability to adhere to discharge plans: communication of instructions, psychosocial factors (financial considerations, shame and guilt, stigma and mental health), access to follow-up care, and pediatric to adult care transitions.

Conclusion: This study describes the patient experience with the communication of discharge instructions, as well as factors affecting adherence post-ED discharge for hyperglycemia. Our findings suggest four strategies that could improve the patient experience, improve adherence to discharge plans, and potentially decrease the frequency of recurrent ED visits for hyperglycemia.

Keywords: Diabetes, Discharge barriers, Hyperglycemia

\section{P094}

A comparison of diabetic ketoacidosis treatment protocols from Canadian adult emergency departments

D. Zheng, BSc, S. Iskander, BSc, MSc, K. Amin, MD, R. Valani, MD, J. Yan, BSc, MD, MSc

Schulich School of Medicine and Dentistry, London, ON

Introduction: Diabetic ketoacidosis (DKA) is a common acute preventable life-threatening complication of poorly controlled diabetes mellitus contributing to considerable mortality and long-term morbidity when not properly managed. There is evidence that use of standardized treatment protocols improves patient outcomes in the emergency department (ED) for a variety of conditions, but variability in adult DKA treatment protocols has not previously been assessed across Canadian EDs. The objective of this study was to compare DKA treatment protocols from adult EDs across Canada to highlight similarities and inconsistencies in the management of this serious presentation.

Methods: ED chiefs and researchers in Canada were solicited for their treatment protocols used to guide acute management of DKA in their EDs. Information regarding the initial fluid replacement, maintenance fluid and adjustment based on blood glucose, potassium replacement, insulin therapy and adjustment, and sodium bicarbonate administration was abstracted for each protocol. Each of these categories were collated in a table and analyzed for overall similarities and differences.

Results: 59 sites (35 urban, 24 rural) across Canada provided a response (71.2\% response rate), with no routine protocol use for 4 urban centres. Similarities in treatment protocols included the amount of potassium replacement, initial dosage of intravenous insulin, and instructions for switching to subcutaneous insulin. Variability in protocols was noted in the rate, amount, and type of initial resuscitative fluid given $(0.5-2 \mathrm{~L}$ of saline or Ringer's lactate (RL) over $15 \mathrm{~min}$ to $2 \mathrm{~h}$ ), the criteria determining the amount given (age, comorbidities, volume status, hypernatremia), the defined value ranges for hypo/hyperkalemia, and the threshold of blood glucose at which to add dextrose to maintenance fluid. $7.5 \%$ of protocols recommended RL for initial fluid resuscitation. Regarding bicarbonate administration, most protocols $(59.3 \%)$ either advised against or excluded it, while $3.7 \%$ recommended consideration of bicarbonate use at $\mathrm{pH}<6.9,33.3 \%$ at $\mathrm{pH}<7$, and $3.7 \%$ at $\mathrm{pH} \leq 7.1$.

Conclusion: This is the first comparison of adult DKA treatment protocols in Canada. While several common approaches were identified, variability was found in initial fluid boluses, maintenance fluid rates and contents, and role of bicarbonate, necessitating further study to guide optimal management strategies and highlight differences in clinical outcomes from these guideline variations.

Keywords: Diabetic ketoacidosis, Emergency department, Standardized protocols 
On behalf of the CAEP Research Committee, it is our pleasure to introduce the dedicated team of volunteers who made CAEP's 2020/21 Grant and Abstract Competitions such a huge success. We could not have achieved this without the support of our volunteers and our generous EM Advancement Fund (EMAF) donors.

Sincerely,

Andrew McRae, MD PhD.

\section{CAEP grant reviewers}

Susan Bartels

Bjug Borgundvaag

Alix Carter

Teresa Chan

Tim Chaplin

Lucas Chartier

Warren Cheung

Raoul Daoust

Kerstin de Wit

Ian Drennan

Jason Frank

\section{CAEP abstract reviewers}

David Barbic

Susan Bartels

Bjug Borgundvaag

Sam Campbell

Alix Carter

Teresa Chan

Kavish Chandra

Tim Chaplin

Warren Cheung

Raoul Daoust

Phil Davis

Valerie De Maio

Kerstin de Wit

Paul Dhillon

Ian Drennan

Marcia Edmonds

Waseem ElHalabi

Ken Farion
Jocelyn Gravel

Katrina Hurley

April Kam

John King

Eddy Lang

Shirley Lee

Dan Mayer

Shelley McLeod

Andrew McRae

Wanda Millard

Merril Pauls
Frank Scheuermeyer

Ian Stiell

Maude St-Onge

Brent Thoma

Christian Vaillancourt

Renee Vilneff

Rob Woods

Krishan Yadav

Justin Yan

Peter Zed
Jason Frank

Judah Goldstein

Sophie Gosselin

Jocelyn Gravel

Andrew K. Hall

Tomislav Jelic

Gary Joubert

John King

Edmund Kwok

Eddy Lang

Shirley Lee

Lindsay Heather

Dan Mayer

Tamara McColl

Jesse McLaren

Shelley McLeod

Andrew McRae

Shawn Mondoux
Laurie Morrison

Robert Ohle

Alim Pardhan

Merril Pauls

Marco Sivilotti

Rob Stenstrom

Ian Stiell

Maude St-Onge

Brent Thoma

Jennifer Thull-Freedman

Christian Vaillancourt

Catherine Varner

Renee Vilneff

Rob Woods

Justin Yan

Fareen Zaver

Peter Zed 


\section{Keywords index}

\begin{tabular}{|c|c|}
\hline 2SLGBTQI+ & LO30 P044 \\
\hline Abdomen/pelvis & LO73 \\
\hline Academic medicine & P079 \\
\hline Access to care & P044 \\
\hline Accuracy & LO20 \\
\hline Acute aortic syndrome & LO59, P064 \\
\hline Acute asthma & P041 \\
\hline Administrative data & P064 \\
\hline Admission & MP22 \\
\hline $\begin{array}{l}\text { Advanced cardiovascular life } \\
\text { support }\end{array}$ & P056 \\
\hline Advanced care paramedics & P056 \\
\hline Advanced care planning & P013 \\
\hline Adverse events & LO54 \\
\hline Agitation & PL01 \\
\hline Airway & LO48 \\
\hline Alcohol & MP10, MP11 \\
\hline Ambulance & $\mathrm{LO} 24$ \\
\hline Analgesia & P030 \\
\hline Analgesics & MP13 \\
\hline Anemia & P065 \\
\hline Ankle fracture & LO41 \\
\hline Antibiotic & MP24, P039, P075 \\
\hline Antibiotic stewardship & P036 \\
\hline Anticoagulation & PL02, LO38, LO60 \\
\hline Anxiety & P081 \\
\hline Anxiolysis & LO39 \\
\hline Appendicitis & P012 \\
\hline Arrhythmia & P051 \\
\hline Artificial intelligence & $\mathrm{LO} 25$ \\
\hline Assessment & LO67, MP20 \\
\hline Asymptomatic bacteriuria & P014 \\
\hline Atrial fibrillation & LO54, LO60 \\
\hline $\begin{array}{l}\text { Automated external } \\
\text { defibrillators }\end{array}$ & P033, P034 \\
\hline Barriers and facilitators & P002 \\
\hline Behavior change & P083 \\
\hline Benign headache & P042 \\
\hline Bleeding & LO69 \\
\hline Bronchiolitis & $\mathrm{LO} 2$ \\
\hline Burnout & LO44, P081 \\
\hline Bystander CPR & LO61 \\
\hline Canadian c-spine rule & PL03 \\
\hline Cancer & LO45 \\
\hline Cannabis & LO09 \\
\hline Cannabis ingestions & MP8 \\
\hline Cardiac arrest & LO40, MP27, P052, P056 \\
\hline $\begin{array}{l}\text { Cardiopulmonary } \\
\text { resuscitation }\end{array}$ & P052 \\
\hline Cardioversion & LO54 \\
\hline Care gap & LO19 \\
\hline Catalytic effect & LO66 \\
\hline CCFP(EM) & P063 \\
\hline Cellulitis & MP6 \\
\hline Checklist & MP26 \\
\hline Chest pain & LO56,LO57 \\
\hline $\begin{array}{l}\text { Chest radiograph } \\
\text { interpretation }\end{array}$ & MP15 \\
\hline Children & LO02, LO08 \\
\hline Clinical decision making & P014 \\
\hline Clinical pathway model & LO19 \\
\hline Clinical practice guidelines & MP7, P082 \\
\hline Clinical workflow & MP3 \\
\hline
\end{tabular}

$\begin{array}{ll}\text { Clinician experiences } & \mathrm{P} 043 \\ \text { Coding } & \mathrm{P} 003 \\ \text { Cognitive load } & \text { LO62, LO63, MP15 } \\ \text { Cold injury } & \text { LO42 } \\ \text { Communication tools } & \text { LO26 } \\ \text { Community } & \text { P030 } \\ \text { Community paramedicine } & \text { LO23 } \\ \text { Competency based medical } & \text { MP16, MP17, P023, P045, P078 }\end{array}$
education

Competency by design $\quad \mathrm{P} 018$

Compression-only CPR LO65

Computed tomography LO73, MP1

Computed tomography LO71, MP2

angiography

Computerized tomography $\quad \mathrm{P} 011$

Conflict of interest P082

Consultation P061

Continuing professional MP18

development

Conventional troponin LO55

Corneal abrasions, topical LO46

analgesia

Coronavirus

Coronavirus disease $2019 \quad$ LO58

COVID

COVID-19

COVID-19 pandemic

CPR

CPR/AED training

Critical care

Crowding

CTAS

Cultural safety

Decision

Decision rule

Delays

Delirium

Delirium recognition

Design

Diabetes

Diabetic ketoacidosis

Diagnosis

Diagnostic imaging

Dilatation and curettage

Directive

Disaster medicine

Disaster response

Discharge barriers

Distance learning

Dizziness

Documentation

Domestic violence

Dopamine antagonist

Downtime

Early pregnancy

complications

ED patient flow $\quad$ LO28

ED triage LO28

Electronic medical records $\quad$ MP4, P069

Emergency

LO03, P087 
Emergency department

Emergency department cardiac monitors

Emergency department

crowding

Emergency department flow

Emergency department

laboratory testing

Emergency department

workers

Emergency medical services

Emergency medicine

Emergency physicians

End-of-life

End-tidal $\mathrm{CO}_{2}$

Entrustable professional

activity

Epiglottitis

Equity

Equity advocacy

Faculty development

Family medicine

Febrile infants

Febrile neutropenia

Fentanyl

Financial transparency

First Nations

FOAM

Force

Fracture

Fractures

Frequent emergency

department users

Frequent users

Frostbite

Functional outcomes

Gastrointestinal illness

Gender equity

Gender variation

Geriatric emergency

medicine

Geriatrics

Global health partnerships

Goals of care discussions

Grand rounds

Head injury

Head trauma

Headache

Health communication design

Health equity

Health professionals

Health systems

Healthy children

Heat

Helicopter emergency

medicine

High-sensitivity troponin

Homelessness

Homemade CPR trainer

Human trafficking

Hydronephrosis

Hyperglycemia

Hyperoxia
LO13, LO35, LO45, LO52, MP14, MP24, P001, P024, P039, P046, P049, P062, P065, P090, P094

P010

P010

LO27

LO16

P026

$\mathrm{P} 031, \mathrm{P} 046$

LO30, LO44, P016, P032, P077

LO21, MP25

$\mathrm{P} 043$

MP27

MP16, MP17

LO70

$\mathrm{P} 058$

P067

LO67, P078

MP19

$\mathrm{P} 022$

LO17

LO29, MP12

P028

$\mathrm{P} 058$

P068

$\mathrm{P} 004$

$\mathrm{P} 080$

LO08

$\mathrm{P} 020$

MP5

LO42

LO08

P088

P079

MP25

$\mathrm{P} 047$

P011

P015

$\mathrm{P} 013$

$\mathrm{P} 008$

PL02

LO38

P090

$\mathrm{P} 083$

LO30

LO14

MP5

LO17

$\mathrm{P} 003$

LO72

LO55

LO24

LO65

P018

LO32

MP9, P093

MP23

$\begin{array}{ll}\text { Hypotension } & \text { LO74 } \\ \text { Iloprost } & \text { LO42 } \\ \text { Individualized care plans } & \text { P020 } \\ \text { Inferior vena cava } & \text { LO74 } \\ \text { Informal learning } & \text { LO61 } \\ \text { Information technology } & \text { MP4 }\end{array}$

failure

Innovations in EM education

LLO49 LO50, LO51O61, P009, P015, P018, P019, P063, P066, P067, P068, P071, P073, P083, P089

MP3

Instant messaging

Intensive care unit

Interdisciplinary

P021

Interdisciplinary health team

Interfacility transfer

P089

P048

$\mathrm{P} 072$

Interfacility transport

P029

Interprofessional relations $\quad$ P061

Intimate partner violence $\quad \mathrm{P} 054$

Intracranial hemorrhage PL02

Intubation

Ketamine

Knowledge translation

Laceration

LO12, LO48, P048, P060,

P092

PL01, P004

P066

LO39

$\begin{array}{ll}\text { Law enforcement personnel } & \text { P001 } \\ \text { Learning analytics } & \text { P078 }\end{array}$

Legalization MP8

Living network meta-analysis $\quad$ LO47

Long-term care

Low value care

Lung-protective

Mace

Machine learning

Management variation

Medical clearance

Medical education

Mental health

Metered-dose inhaler and spacer

Migraine

Mild traumatic brain injury

Misdiagnosis

LO23, LO34, P062

LO18

MP23

LO57

LO25

LO01

$\mathrm{P} 027$

LO50, MP15, MP21, P073

LO31, MP11

P074

MP28, P090

PL04

LO59

$\mathrm{P} 032$

P021

LO66

MP12

P076

LO02

LO71

P054

LO37

LO64, P045

P080

LO63

MP6

P057

LO29, MP13

P071

MP1

P037, P038

LO32, MP22

LO57

LO29, MP12, MP13

LO10 
Paediatric

Paediatric urinary tract

infection

Paediatrics

Pain

Pain assessment

Palliative care

PALS

Pandemic

Paramedic

Patient assessment

Patient care

Patient experience

Patient flow

Patient perceptions

Patient safety

Patient volumes

Patient-reported outcome

measures

Pediatric

Pediatric emergency

medicine

Pediatrics

Predictors

Pet therapy

Photovoice

Physician communication

Physician financial wellness

Physician wellness

Physicians

Pneumonia

Podcast

Podcast evaluation

Podiatry consult

Point-of-care ultrasound

Poisoning

Police

Population level study

Positive deviance

Postal survey

Post-concussion symptoms

Postpartum complications

Practice variation

Practitioner health

Prediction

Prediction scores

Prehospital

\section{Prehospital care}

Prepatching

Prescribing

Primary healthcare provider

Procedural sedation

Prognosis

Program evaluation

Protocol implementation

Public access defibrillation

Public safety

Pulmonary embolism

Quality improvement

Quality improvement and patient safety

LO39
LO15
P055
P055, P080
LO33
MP14, P043
LO68
P081, P088
P031
P017
P091
LO06, LO26, P041, P042
LO14, P059
P091
P050
LO73, P049
P085

LO03, LO68, P075, P086

LO07, MP18

LO05, MP8

$\mathrm{P} 047$

LO06

P041, P042

$\mathrm{P} 061$

P028

P028

P069

P036

MP21, P068

MP21

$\mathrm{P} 024$

LO68, LO74, LO75, MP20

LO09

P001

LO05

P008

$\mathrm{P} 037$

PL04

P087

P014

$\mathrm{P} 032$

LO25

LO69

PL03, LO22, LO53, P004,

P050, P055

P029, P072

P029

P057

LO28

$\mathrm{P} 030$

LO75

LO67

$\mathrm{P} 025$

P033, P034

$\mathrm{P} 034$

MP2

LO50, MP2

LO10, LO11, LO12, LO13,

LO14, LO15, LO16, LO17,

LO18, LO27, MP11, MP3, P012,

P020, P024, P025, P059, P060,

P062, P074, P084, P085
Quality of life

Randomized clinical trial

Randomized controlled trial

Rapid response team

Reactive airways disease

Realist evaluation

Referral and consultation

Remote learning

Remote monitoring

Renal colic

Renal replacement therapy

Residency education

Residents

Resource stewardship

Respiratory illness

Responsibility to care

Resuscitation

Rib fractures

Risk

Risk score

Risk stratification

Rural

Sars-cov-2

Scene times

Schools

Scoping review

Screening

Sentinel visit

Severe bacterial infections

Sexual assault

Sexually transmitted infections

Shared mental model

Sickle cell

Sickle cell disease

Simulation

Simulation training

Skin and soft tissue infections

Spinal immobilization device

Spontaneous abortion

ST Elevation Myocardial

Infarction

Standardized protocols

STEMI

Stroke

Substance psychosis

Substance use

Substance-related disorders

Successful habits

Supraglottitis

Syncope

Systematic review

Tachycardia

Teaching medical students

Telemedicine

Theoretical domains

framework

Throughput

Tranexamic acid

Transfer

Transfusion

Transition to practice

Transitions in care

Trauma

Trauma teams
$\mathrm{P} 077$

LO36

PL01, PL03

P048

P074

P053

LO07

LO65

LO1 1

LO32, P040

$\mathrm{P} 021$

$\mathrm{P} 023$

LO62

MP1

P088

LO21, P026

LO62, MP26

P076

P057

LO04

LO56

MP19

LO13

LO72

$\mathrm{P} 002$

LO09, P044

LO35

MP9

P022

P027, P054, P070

LO16

P066

P025

P091

LO49, LO51, MP19, P009, P092

LO63, P073

MP24, P039

$\mathrm{LO} 22$

LO01, MP25

LO58

P094

LO53

LO60, LO71, P031

P005, P006, P007

LO31

LO07

MP16

LO70

LO52, LO55

LO06, LO44

P051

$\mathrm{P} 071$

LO03, LO34

MP17

P053

LO36

P086

$\mathrm{P} 065$

P063

MP5

LO05, LO10, LO22, LO37, LO40, LO43, P050

LO66 
Traumatic arrest

Traumatic brain injury

Treatment

Triage

Troponin

Trustworthiness

Ultrasound

Undergraduate medical education

Uptriage

Urinary tract infection

Urine bag collection

Urine culture

Validation

LO75
LO36, LO38
LO47
LO04, LO20, LO33, P017,
P058
LO52
MP7
LO72, P076
MP20
P017
P075
LO15
LO18
P003

MP28

Venous thromboembolism LO41

Ventilation MP23

Ventricular fibrillation $\quad$ MP27

Vertigo P035

Video review LO37

Viral illness $\quad \mathrm{P} 022$

Virtual LO49

Virtual education P015

Virtual rounds $\quad$ P019

Visit P087

Wait times $\quad$ LO26, P059

Withdrawal MP10

Workplace-based assessment LO64, P023, P045 


\section{Author index}

Abdulaziz, K. E.

Abolhassani, S.

Abou-Setta, A. M.

Al Lawati, K.

Al Maqbali, S.

Al Rimawi, $\mathrm{H}$.

Alaazi, D.

Alchi, S.

Alder, R. N.

Al-Grigri, O.

Al-Haimus, F.

Ali, $\mathrm{H}$.

Ali, M.

Ali, M. M.

Ali, S.

Allain, D.

Allan, K. S.

Allen, L. M.

Alnaji, F.

Alrimawi, H.

Alrobaian, S.

Alsenchuk, S.

Al-Yousif, N.

Amin, $\mathrm{K}$.

Ananthanarayanan, S.

Anderson, P.

Andolfatto, G.

Andruchow, J.

Anjum, O.

Archambault, P. M.

Armstrong, D.

Askin, N.

Aspler, A.

Atkinson, $\mathrm{P}$.

Atzema, C. L.

Austin, E.

Austin, $\mathrm{M}$.

Austin, P. C.

Avlijas, T.

Azzam, D.

Baassiri, H.

Bandi, V.

Barbic, D.

Barbic, S. P.

Barnabe, C.

Bartlett, E.

Barton, A.

Bartoszko, J.

Baweja, S.

Bdira, A.

Beaubien, M.

Beck, C.

Beique, M.

Bentley, M.

Berthelot, S.

Besserer, F.

Beyene, $\mathrm{T}$.

Bhangu, A.

Bharadia, M.

Bhatia, M.
$\mathrm{P} 037$

P001

LO28

LO36

LO36

LO36

P062

P051

LO28

$\mathrm{P} 022$

LO38, O60

LO21

LO69

P080

LO06, LO07, O08, O21, O39,

P026

LO02

$\mathrm{P} 002$

LO66

MP18

MP16, MP17

LO74

LO11

LO28

P094

MP7

P020

PL01, P004

LO42, LO56

MP16, MP17

PL04, P081

LO46

LO28

P015

LO74, LO75, P016, P056

PL02, LO41

LO16

P080

PL02

LO35

P093

$\mathrm{P} 003$

$\mathrm{P} 078$

PL01, LO31, P004, P005, P006,

P007, P032, P077

PL01, LO31, P004, P005, P006, $\mathrm{P} 007$

P058

MP3

$\mathrm{P} 020$

LO47

LO60

MP18

P085

LO17

MP4

$\mathrm{P} 032, \mathrm{P} 077$

PL04, LO28, P026

P004

$\mathrm{P} 015$

LO37

LO08

P008
Bhatt, M.

Bigham, B.

Bignell, $\mathrm{M}$.

Bilgasem, A.

Bilic, M.

Bill, L.

Billinkoff, C.

Birchall, I.

Bischoff, T.

Boggild, A.

Boissonneault, E.

Booth, R.

Boreskie, P.

Borgundvaag, B.

Bouchard, M.

Boucher, V.

Boulos, M.

Bourdeau, V.

Bourque, C.

Bouwsema, M.

Boyd, H.

Bradley, R.

Brain, P.

Braund, $\mathrm{H}$.

Bravo, G.

Brazier, J

Brignardello-Petersen, R.

Brimmer, B.

Brindley, P. G.

Brisebois, A.

Brown, E.

Brown, R.

Brown, S.

Bryan, J. M.

Buchel, T.

Burcul, I.

Burgess, J.

Burke, K.

Burns, K.

Burstein, B.

Burwash-Brennan, T.

Butchart, S.

Butcher, J.

Cada, M.

Calder, B.

Calder, S.

Calder-Sprackman, S.

Cameron, P.

Campbell, S.

Carciumaru, $\mathrm{R}$.

Carey, R.

Carly, H.

Carlyle, T.

Carpenter, C.

Carter, A.

Carter, J.

Carter, $\mathrm{K}$

Castonguay, V.

Caswell, J.

Ceccacci, A.

Chakraborty, A. T.

Chalklin, K.

Chan, T. M.

Chandra, K.
LO39

P044

LO16

LO65

MP21, P009

$\mathrm{P} 058$

LO16

LO26

P029

LO11

LO65

LO55

LO74

PL02, LO41, MP24, P039, P047

MP4

PL04, P047

MP21

P026

P026

MP16, MP17

LO34

P010

LO01, MP25

MP15

LO68

P030

LO47

LO15

P052

MP14, P043

LO54

P031

P030, P086

P015

LO28

LO24

LO34

P055

LO75

P026

LO75

LO17

P011

LO17

P020

LO19

P025

PL04

LO20

$\mathrm{P} 022$

P068, P078

P037

LO11

P082

P031

LO60

MP19

P026

LO59

P083, P085

LO31, P005, P006, P007

P084

LO51, LO67, MP16, MP17,

MP21, P078, P079, P081

LO74, P056 
Chang, M.

Chaplin, T.

Charette, $\mathrm{M}$.

Chartier, L.

Chaudhari, H.

Chauny, J.

Chen, A.

Chen, J.

Chen, K.

Cheng, I.

Cheng, W.

Cheskes, L.

Cheskes, S.

Cheung, K.

Cheung, W.

Chhabra, S.

Chignell, M. M.

Chin, A.

Chin, K.

Chisholm, D.

Cho, R.

Chochinov, A.

Choi, J.

Chung, D.

Chun, S.

Ciacco, B.

Clapham, G.

Clayton, N.

Clemens, K.

Clément, J.

Clemente, M.

Clerc, $\mathrm{P}$.

Cole, V.

Columbus, M. P.

Conlon, M.

Cook, C.

Copstein, L.

Cortel, M.

Côté, A.

Couperthwaite, A.

Coyle, N.

Creasor, E.

Cristall, N.

Crooks, S. M.

Crump, S.

Culligan, $\mathrm{C}$.

Cummings, G.

D Trottier, E.

Da Luz, L.

Dahn, T.

Darkwa, M.

Dashi, G.

Davies-Schinkel, C.

Davis, M.

Davis, S.

de Guise, E.

de Wit, K.

DeBiasio, C.

Dell, E.

Deng, W.

Denize, K.

Dennett, L.
LO11

LO62, LO63, LO66

PL03, LO22

LO13, LO50, LO58, P048, P049

$\mathrm{P} 012$

PL04

MP5

P084

LO19

LO14

MP28

$\mathrm{P} 013$

LO22, MP27

LO26

LO35, LO49, LO64, LO67,

MP16, MP17, MP20, MP26,

P013, P023, P045, P063

MP6

P047

P079

P048

LO43

MP12

LO28

MP3, P014

LO05

LO16, P015

LO14

LO19

LO38, LO60, LO69

$\mathrm{P} 003$

PL04

P027

P026

$\mathrm{P} 016$

MP9, P093

LO59

P008

LO28

MP28

LO21, P026

LO43

LO14

LO70

LO05

LO42

LO13, P048

LO13

$\mathrm{P} 062$

LO21, P026

LO10, LO37

P016

P091

LO16

LO44, MP7, P030, P082

LO53

LO33, P017

PL04

PL02, LO34, LO38, LO41,

LO60, LO69, P081

LO19, MP20

P018, P067

LO24

$\mathrm{P} 070$

LO06
Desaulnier, P.

Deutscher, J.

Dewhurst, N.

Dharamsi, A.

Dida, J.

Dingwall, M.

Dionne, R.

Dong, K. J.

Dorian, $\mathrm{P}$.

Douglas, S.

Douma, M.

Doupe, $\mathrm{M}$.

Dowling, S.

Doyle-Waters, M. M.

Dragoman, A.

Drake, K.

Drendel, A.

Drennan, I.

Drew, D.

Duarte Wisnesky, U.

Dudek, N.

Duggan, S.

Dullemond, K.

Dunfield, R.

Dunnewold, N.

Durr, K. M.

Dutton, D.

Dy, J.

Eagles, D.

East, C.

Edwards, J.

Eissa, M.

Elliot, E.

Elliott, S.

Ellis, B.

Eltorki, M.

Elwi, A.

Elzinga, J.

Emond, M.

Endres, $\mathrm{K}$.

Evans, C.

Everett, K.

Ewa, V.

Exner, K. D.

Fantauzzi, M.

Farina, L.

Faris, P.

Farquharson, S.

Felkar, B.

Fell, D.

Fergusson, D.

Fernandes, J.

Fernandes, R.

Fidgen, D.

Fillion, L.

Finlayson, A.

Fok, P.

Fontaine-Pagé, E.

Foohey, S.

Fournier, K.

Franc, J.

Frank, J.

Fraser, J.

Freedman, C.
P026

P018

P084

LO13, P066

MP21

MP7

PL03

MP21

$\mathrm{P} 002$

P069

P033, P034, P052

LO28

LO18, LO73, MP1, P019, P075

MP10

P059

LO34

LO08, LO39

MP27

MP26

LO20

$\mathrm{P} 023$

MP14, P043

LO12, LO48, P092

LO74

LO03

$\mathrm{P} 001$

LO74, P016

LO19

LO35, LO54, MP6, P047, P051, $\mathrm{P} 054, \mathrm{P} 080$

$\mathrm{P} 020$

LO28

P021

LO14

LO02, LO06, LO09

P011

$\mathrm{P} 012, \mathrm{P} 022$

MP14, P043

LO73, MP1, P053

P047

$\mathrm{P} 023$

LO40

LO41

$\mathrm{P} 062$

$\mathrm{P} 024$

LO17

P092

P062

LO69, P020

LO06

$\mathrm{P} 070$

PL03

LO48

LO02

P031

LO14

LO52

P016

P026

LO51

LO25

LO20

LO64, P013, P045

LO74, P056

LO10, LO37 
Freedman, S. B.

Fremes, E.

Frenette, J.

$\mathrm{Fu}, \mathrm{M}$.

Fung, T.

Gagnon, C.

Galbraith, R.

Gangatharan, H.

Garrido Clua, M.

Gaucher, N.

Gaudet, L.

Gauri, A.

Gawaziuk, J.

Ge, L.

George, M.

George, S.

Germini, F.

Ghader, Y.

Ghate, G.

Gilic, F.

Ginocchio, G.

Girdhari, R. P.

Glazer, P.

Glicksman, R. A.

Golden-Plotnik, S.

Goldman, R.

Goldstein, J.

Gourlay, K.

Grafstein, E.

Graham, C.

Grandjean-Blanchet, C.

Grant, L.

Granton, J.

Gray, S. H.

Graystone, A.

Green, M.

Greene, J.

Grewal, K.

Griesdale, D.

Grunau, B.

Guertin, H.

Guirguis, S.

Gupta, S.

Gupta, S.

Guscott, J. R,

Gutiérrez, G,

Guyatt, G,

Hacker Teper, M,

Hair, $\mathrm{H}$,

Halas, G,

Hall, A. K.

Hall, E.

Hamilton, J.

Hamilton, N.

Hanel, E.

Hann, J.

Hansen, M.

Harper, K.

Harper, M.

Harris, T.

Hartling, L.

Hartmann, R.

Harton, F.

Hartwick, M.

Hatam, E.
$\mathrm{LO} 02$

P018, P067

PL04

P025

P075

P092

LO03

MP4

MP14

LO21, P026

LO02, LO06, LO09

LO07

LO05

LO47

P062

MP11

LO69

P083, P085

MP7, P027, P028, P082

LO49

LO16, P083, P085

P084

LO13

P029

LO08

P026

P031, P047

$\mathrm{P} 040$

LO31

P036

LO17

LO33, P017

LO11

P081

P030

P051

P031

PL02, LO41, LO45

LO48

PL01, P004

P032, P077

LO13

P041

LO16, P083, P085

LO49

LO62, LO63

LO47

LO50

P062

LO28

MP15, MP16, MP17

PL03

P055

LO16

P009

LO07

P033, P034

LO70

LO11

LO75

LO02, LO06, LO09

MP8

$\mathrm{P} 024$

P037, P038

P060
Hayawi, L.

Hayman, K.

Haynes, L.

Hayward, J.

Hegdekar, M.

Heimerl, M.

Henson, A.

Herman, D.

Hewitt, M.

Hickey, C.

Hickey, M.

Hill, N.

Hogue, K.

Holroyd, B. R.

Holroyd-Leduc, J.

Homier, V.

Honer, W. G.

Horner, D.

Hossain, R.

$\mathrm{Hu}, \mathrm{G}$.

$\mathrm{Hu}, \mathrm{X}$.

$\mathrm{Hu}, \mathrm{Y}$.

Huang, P.

Hulme, J.

Hussein, A.

Ibrahim, T.

Innes, G. D.

Isaackz, S.

Ishimwe, A.

Iskander, S.

Islam, A.

Izcovich, A.

Janiver, K.

Jaskolka, J.

Jefkins, T. T.

Jelic, $\mathrm{T}$.

Jemitola, S.

Jen, $M$.

Jeyaraman, M. M.

Joe, R.

Johnson, D.

Johnson, D. W.

Jones, J.

$\mathrm{Ju}, \mathrm{C}$.

Jung, $\mathrm{H}$.

Kaban, G.

Kallergis, D.

Kam, A.

Kang, S. J.

Kapend, P.

Kapur, P.

Kareemi, H.

Kearney, $\mathrm{H}$.

Kelliher, B.

Kelly, P.

Kennedy, W.

Kestenbom, I.

Khalid, M.

Khamis, A.

Killam, R.

Kim, A.

Kim, J.

Kirby, M.

Kirkham, A.
MP18

P018, P067

P035, P089

LO29, MP13

LO52, LO55

P070

LO61

P036

LO50

P038

P037, P038

P041, P042, P043

LO09

P058

P062

MP4

PL01, LO31, P004, P005, P006,

P007

LO41

LO24

P030

MP5

LO60, LO69

LO52, LO55

P067

P089

MP24, P039

LO29, LO32, MP13, P040

LO11

LO55

P094

$\mathrm{P} 040$

LO47

P058

LO71, MP2

$\mathrm{P} 002$

LO72, P076

P091

$\mathrm{P} 031$

LO28

MP12

LO46

$\mathrm{LO} 02$

LO13

LO33, P017

P049

LO57

P059

$\mathrm{P} 012$

LO45

MP7

P061

LO25

LO44

MP11

LO23

P089

LO15

MP21, P030, P086

LO47

LO27

LO40

P065

P091

LO07 
Kirkland, S.

Kirubarajan, A.

Kirwan, C.

Kisilewicz, M,

Kiss, A.

Klassen, T. P.

Koh, J. J.

Kokoski, C.

Koziak, M.

Krebs, L. D.

Kruhlak, M.

Kruse, M.

Krywenky, A.

Kum, E.

Kumar, K.

Kumar, S.

Kumbhare, D.

Kwok, E. S.

Kwong, J. L.

Lafave, $\mathrm{H}$.

Lagacé, M.

Lalande, E.

Lam, W.

Landreville, J. M.

Lane, D.

Lane, D. J.

Lang, E. PL04.

Latimer-Cheung, A. E.

Lau, T.

Laviolette, A.

Lavoie, B.

Le Sage, N.

Leach, A.

Leafloor, C. W.

Leduc, S.

Lee, D.

Lee, J.

Leppard, J.

Leroux, Y.

Lewis, D.

Li, M.

Li, W.

Lien, $\mathrm{K}$.

Lim, R.

Lin, K.

Lin, $\mathrm{K}$.

Lin, $\mathrm{S}$.

Linton, J.

Liu, S. L.

Liu, T.

Lloyd, T.

Loch, T.

Locquiao, J.

Logsetty, S.

Lonergan, K.

Lou, W.

Loubani, O.

Lun, R.

Luongo, A.

Luterman, $\mathrm{M}$.

Lynch, $\mathrm{T}$.

Ma, M.
LO20, MP14, P043

LO46

LO60, LO69

P057

P047

LO02

MP10

P084

LO07

P041, P042, P043

MP14

P044

MP26

LO47

LO39

MP6

P082

LO19, P010, P025

MP27

LO03

P026

LO75

LO49

LO64, P045

P046

LO32

LO01, LO03, LO27, LO43,

MP11, MP25, MP8, P024, P035, P047, P053, P062, P082, P088

\section{LO26}

P040

LO44

P026

PL04, LO52, LO55

LO57

LO52, LO55

LO23

LO50, P048, P049

PL04, P047

P063

P031

LO74

P092

P050

LO30

P028

P071

MP12

$\mathrm{P} 002$

$\mathrm{P} 051$

P093

P016

P018

P052, P053

LO11

LO05

LO01, LO32, MP11, MP25,

$\mathrm{P} 024$

P049

MP22, P021

P090

P005, P006, P007

LO44

LO68

P048
Ma, Z.

Macarthur, C.

MacDonald, Z.

Mace, C. A.

MacEwan, W.

Mackey, L.

Macleod, J.

Mah, A.

Mal, S.

Malczewska, M.

Malhotra, D.

Mara, C.

Mardoquio, M.

Marks, N.

Martin, L.

Mason, E.

Masood, S.

Mathieu, M.

Matukas, L.

Maxwelll, B.

McConnell, M.

McGraw, M.

McIntyre, L.

McIsaac, S.

McKinney, M.

McKinnon, M.

McLane, P.

McLaren, J.

McLeod, B.

McLeod, S. L.

McMurray, L.

McRae, A. D.

Mekwan, J.

Mela, M. A.

Melady, D.

Mercier, É.

Mercuri, M.

Meyer, J.

Michaelson, S.

Miller, M.

Mireault, J.

Mirza, A.

Mittmann, N.

Moayedi, Y.

Moe, J.

Moir, M.

Mok, G.

Molnar, F.

Mondal, D.

Mondoux, S.

Mont, $\mathrm{K}$.

Monteiro, S.

Moore, L.

Morden, D.

Morra, M.

Morris, J.

Moskalyk, M.

Moustapha, A.

Mubasher, M.

Muftah, A.

Mukarram, M.

Muldoon, K. A.

Munene, A.
LO44

LO22

P054

$\mathrm{P} 055$

PL01, P004

P058

P092

LO57

P060

MP10

LO44

MP19

LO16

LO1 1

LO57, MP16, MP17

LO50

LO11, P049

$\mathrm{P} 025$

LO16

MP9

$\mathrm{P} 023$

P056

P037, P038

LO59, LO61, P008, P064

P057

LO19

MP5, P041, P058, P062

LO58

PL03, LO22

PL02, LO41, LO45, LO47, MP24, P039, P047, P082

MP26

LO32, LO52, LO55, LO56,

MP5, P046, P047

P056

P001

$\mathrm{P} 047$

PL04, LO52, LO55

LO34, LO71, MP2

LO20

LO14

LO68

LO28

$\mathrm{P} 022$

PL02

LO11

MP10, MP12

LO08

$\mathrm{P} 050$

LO35

P078

LO34, LO50, P059

P020

LO51, LO67

PL04

P060

MP15

MP7, P082

LO61, LO65

LO57

MP21

P061

LO55

P054, P070

P062 
Murdock, M.

Nagji, A.

Nath, A, MP28.

Nathens, A.

Nemnom, M.

Neveu, X.

$\mathrm{Ng}, \mathrm{V}$.

Niaz, S.

Nickonchuk, T.

Nikouline, A.

Nirmalanathan, K.

Nolan, B.

Nolan, S.

Notario, L.

O'Brien, M.

ODochartaigh, D.

OHalloran, K.

Ohle, R.

O'Neil, E.

O'Neill, B.

Onotera, K.

O'Rielly, C. M.

Orvold, J.

Ospina, M. B.

Ossip, M.

Ostrow, O.

O'Sullivan, F.

Ouellet, M.

Oura, E.

Oyedokun, T. O.

Pace, J.

Pageau, P.

Paige, B.

Paluck, F.

Panju, M.

Pannell, D.

Papaioannou, A.

Pardhan, A.

Pardhan, K.

Parotto, M.

Parpia, C.

Pasay, D.

Patel, D.

Paterson, Q.

Patey, A. M.

Patocka, C.

Peddle, M.

Pernica, J.

Perri, P.

Perry, J. J.

Peterson, A.

Petrosoniak, A.

Pettit, C.

Phalpher, P.

Pham, C.

Philips, H.

Phillips, A.

Picard, C.

Pisani, K.

Pishe, T.

Pizzola, C.

Plint, A. C.

\section{MP21}

LO5, P009

MP6, P063

LO10, LO37

LO52, LO54, LO55, P051, P080,

P090

PL04

LO30

LO60, LO69

LO18

LO16

P048

P029, P050, P072

LO31

LO10, LO37, P074

LO49, MP26

P052

LO34

LO35, LO59, LO61, LO65,

LO70, MP6, P008, P064, P073

$\mathrm{P} 002$

MP14, P043

LO72

LO56

LO57

MP5

LO71, MP2

LO15, LO17

MP12

PL04

P065

P001, P061

P060

MP20

P064

LO15

LO34

LO37

LO34

P059

LO10, LO14

P048

LO50, P066

LO18

MP28

MP16, MP17

$\mathrm{P} 013$

LO67, P035, P053

P029

$\mathrm{P} 022$

$\mathrm{P} 048$

PL04, LO49, MP28, P037, P038, P047, P063, P090

LO18, P019

LO36

P059

LO71, MP2

LO74

P004

LO10

$\mathrm{P} 052$

LO61

P056

$\mathrm{P} 022$

$\mathrm{LO} 02$
Polsky, Z.

Poonai, N.

Pozgay, A.

Pozzobon, L.

Prpic, J.

Pun, C.

Purssell, R.

Pymento, J.

Qayyum, S.

Rabbani, R.

Radomske, D. J.

Radulovic, N.

RAFF Study Authors

Rajagopal, M.

Rajaram, A.

Ramsden, S.

Rashid, T.

Rathagirishnan, R.

Rauchwerger, D.

Rebinsky, R.

Reid, S.

Reid, S.

Reiter, E.

Ren, K.

Renschler, C.

Rice, C.

Ritchie, K.

Rittenbach, K.

Roberts, L.

Robinson, O.

Rochwerg, B.

Rocklein Kemplin, K.

Rohringer, T.

Rose, L.

Rose, S.

Rosenberg, H.

Rosenfield, D.

Ross, P.

Rosychuk, R. J.

Rowe, A.

Rowe, B. H.

Rullo, E.

Rutledge, G.

Ryan, S.

Saah-Haines, R.

Sabbah, S.

Sabhaney, V.

Sabri, E. PL03.

Sadoway, R.

Salehi, L.

Samarasinghe, N.

Sample, S.

Sampsel, K.

Sarti, A.

Saskin, R.

Sathiaseelan, S.

Savage, D.

Sawadsky, B.

Saxinger, L.

Scheirer, O.

Scheuermeyer, F. X.

Schmidt, H.
P053

LO08, LO39, P055

LO19

LO50

PL03

P073

MP12

$\mathrm{P} 022$

P067

LO28

P068

LO40

LO54

P026

P069

LO69

LO69

LO26

P049

P012

MP18

$\mathrm{P} 062$

LO06

P059

LO12, LO48, P092

P083, P085

P081

$\mathrm{P} 058$

LO57, P011

LO03

LO36, MP23

P032, P077

P036

$\mathrm{P} 047$

P019

LO25, MP6

LO17

LO74

MP5, P058

MP12

LO20, LO28, LO52, LO55,

MP14, P041, P042, P043

$\mathrm{P} 012$

$\mathrm{P} 059$

LO72

MP8

P048

LO39

LO22

MP22

LO71, MP2

MP9

MP16, MP17, MP21

$\mathrm{P} 054, \mathrm{P} 070$

P037, P038

PL03

P049

LO59, P064, P073

P029

LO18

P068

PL01, LO31, LO32, LO48,

LO74, P004, P005, P006, P007,

$\mathrm{P} 040$

MP3 
Schneeweiss, M.

Science, M.

Scott, $S$.

Sellen, K.

Seto, A. V.

Sevcik, W.

Shachar, E. D.

Shadowitz, S.

Shah, A.

Shani, K. M.

Shanmugarajah, A.

Sharif, S.

Sharma, S.

Sharma, T.

Shavadia, J.

Shaw, B. H.

Shefrin, A. E.

Shelton, D.

Shepherd, L. G.

Shimmin, C.

Shkrobot, R.

Sibbald, M.

Sidhu, A.

Sidhu, G.

Sidhu, K.

Siemieniuk, R. A.

Singh, S.

Sivakumar, M.

Sivilotti, M. L.

Skitch, S.

Smith, A.

Smith, K. E.

Smith, M.

Smith, M.

Snider, C.

Snyder, H.

Sobiesiak, A.

Solback, N.

Solh, Z.

Spackman, E.

Spaic, T.

Srajer, A.

Srivastava, S.

Stancati, A.

Stanojev, S.

Stanway, J.

Stauffer, B.

Stein, N.

Stempien, J. E.

Stewart, D.

Stewart, D.

Stiell, I.

Strobel, S,

Sudershan, S.

Suh, K.

Surdhar, I.

Suss, R.

Sutradhar, R.

Suwary, K.

Swain, J.

Szulewski, A.

Taher, A. K.

Talarico, R.
P012

LO17

LO06, LO09

P083, P085

P071, P089

LO28

P072

LO14

LO39

P073

P008

LO36, MP23

PL02

P086

LO57

LO42

MP20

P074

P093

LO28

LO18

LO51

LO11

P004, P005, P006, P007

P059

LO47

LO67

LO08

LO52, LO55

MP23

LO14

P033, P034

P059

P011

P084

P086

P070

P019

P065

P062

P093

LO01, MP25

P069

P059

LO04

LO14

LO06

$\mathrm{P} 012$

P001, P061

P075

MP14

PL03, LO22, LO35, LO54, P051, P057, P090

LO24, P030, P059, P082

\section{$\mathrm{P} 075$}

MP6

P076

LO28

LO41

P032, P077

$\mathrm{P} 031$

LO62, MP15

LO13, LO50P048, P049 P070
Taljaard, M.

Tan, A.

Tardif, P.

Teefy, J.

Tenorio, F.

Teo, V.

Test, G.

Thavorn, K.

Theodoropoulos, J.

Thiruganasambandamoorthy, V.

Thoma, B.

Thomas, S.

Thomas-Boaz, W.

Thompson, C.

Thompson, G.

Tien, $\mathrm{H}$.

Tierney, M.

Tillmann, B.

Tissot, E.

Tolmie, A. D.

Tong, $\mathrm{T}$.

Tran, A.

Tran, U.

Tricco, A. C.

Tromp, M.

Trotter, B.

Trottier, E.

Tse, S.

Tsoutsoulas, C.

Tunji-Ajayi, L.

Turner, L.

Ukrainetz, K.

Upadhye, S.

Vadera, R,

Vaillancourt, C.

Vaillancourt, S.

Valani, R.

Van Heer, S.

Van Manen, M.

VandenBerg, S.

Vandermeer, B.

VanAarsen, K.

VanDusen, J.

Varghese, T.

Varma, M.

Vatanpour, S.

Verhovsek, M.

Verma, A.

Viau, J.

Villa-Roel, C.

Villeneuve, S.

Vincent, M.

Vishnyakova, E.

Voloshin, D.

Vorobeichik, A.

Vujcic, B.

Wahba, J.

Walia, $\mathrm{H}$.

Walker, I.

Wallner, C. F.
PL03, LO22, LO35, LO52,

LO55

LO69, P079

LO28

P055

P092

$\mathrm{P} 074$

LO15

MP6

LO41

LO23, LO52, LO55, P010, P025

LO67, P068, P078

LO75

LO10, LO14, LO37

MP24, P039

$\mathrm{P} 012, \mathrm{P} 026$

LO37

P047

LO10

LO65

P079

P047

P081

$\mathrm{P} 080$

LO28

LO49

MP21

LO39

LO26, MP18

P014

P091

MP27

LO73

LO44, MP7, P030, P044, P082,

P086

P059

PL03, LO22, LO23, LO25,

MP26

LO16, P083, P084, P085

LO71, MP2, MP7, P086, P08,

P094

LO62, LO63, P069

LO08

P088

LO02

LO68, MP9, P093

$\mathrm{P} 033, \mathrm{P} 034$

$\mathrm{P} 003$

$\mathrm{P} 088$

LO73

P091

LO10, LO14

MP28

MP14, P041, P042, P043

LO17

MP16, MP17

P090

P044

P089

LO30, LO53, MP9, P003, P027, P055, P060, P065

$\mathrm{P} 022$

P088

$\mathrm{LO} 42$

LO34, P081 
Walsh, C.

Wang, Y. E.

Wang, D. C.

Wang, D.

Wang, S.

Watson, E.

Weaver, C. G

Webster, R.

Wells, G. A.

Wells, S. T.

Welsford, M.

Weng, V.

Weyman, K.

Whyte, M.

Wiemer, H.

Williams, E.

Williams, S.

Wilson, S.

Winter, $\mathrm{K}$

Wishart, I.

Wolfrom, B

Wong, D.

Wong, $\mathrm{H}$.

Wong, S.

Wong, S. J.

Woo, M.

LO45
MP12
MP3
MP1
P078
P001
MP1, MP8
MP18
LO23
P090
LO34
P085
P084
P004, P005, P006, P007, P032
P016
LO07
P091
LO19
LO34
P040
P069
LO22
PL01, P004
LO34, P073
LO12, P092
LO75, MP20

Wood, T.

Woods, R.

Worster, A.

$\mathrm{Wu}, \mathrm{R}$.

Wyer, L.

Wylie, M.

Yadav, K.

Yan, J. W.

Yang, A.

Yang, E.

Yau, L.

Yau, M.

Yeung, M.

Yilmaz, Y.

Yoo, J. H,

$\mathrm{Yu}, \mathrm{C} . \mathrm{W}$.

$\mathrm{Yu}, \mathrm{H}$.

Zarychanski, R.

Zaver, F.

Zeraatkar, D.

Zheng, D.

Ziegler, N.

Zimmermann, R.

Zouboules, S.
LO64

LO6, MP16, MP17, P078

$\mathrm{P} 012$

LO11

P062

LO01, MP25

LO25, LO35, LO59, MP6, P037, P038, P054, P080

LO52, LO55, MP7, MP9, P003, P082, P093, P094

MP19

MP14

LO53

LO46

MP8

LO51, P078

LO12, LO48, P092

LO46

MP2

LO28

P019

LO47

P094

LO14

LO57

LO03 